San Jose State University

SJSU ScholarWorks

Master's Theses

Master's Theses and Graduate Research

Spring 2010

\title{
Synthesis and Evaluation of Silica Hydride-Based Fluorinated Stationary Phases
}

Kavita Vipool Prajapati

San Jose State University

Follow this and additional works at: https://scholarworks.sjsu.edu/etd_theses

\section{Recommended Citation}

Prajapati, Kavita Vipool, "Synthesis and Evaluation of Silica Hydride-Based Fluorinated Stationary Phases" (2010). Master's Theses. 3785.

DOI: https://doi.org/10.31979/etd.hhsh-4dsr

https://scholarworks.sjsu.edu/etd_theses/3785

This Thesis is brought to you for free and open access by the Master's Theses and Graduate Research at SJSU ScholarWorks. It has been accepted for inclusion in Master's Theses by an authorized administrator of SJSU ScholarWorks. For more information, please contact scholarworks@sjsu.edu. 


\title{
SYNTHESIS AND EVALUATION OF SILICA HYDRIDE-BASED FLUORINATED STATIONARY PHASES
}

\author{
A Thesis \\ Presented to \\ The Faculty of the Department of Chemistry \\ San Jose State University
}

In Partial Fulfillment

of the Requirements for the Degree

Masters of Science

by

Kavita Vipool Prajapati

May 2010 
(C) 2010

Kavita Vipool Prajapati

ALL RIGHTS RESERVED 
The Designated Thesis Committee Approves the Thesis Titled

\title{
SYNTHESIS AND EVALUATION OF SILICA HYDRIDE-BASED FLUORINATED STATIONARY PHASES
}

\author{
By \\ Kavita Vipool Prajapati \\ APPROVED FOR THE DEPARTMENT OF CHEMISTRY \\ SAN JOSE STATE UNIVERSITY
}

May 2010

Dr. Joseph J. Pesek

Department of Chemistry

Dr. Roger Terrill

Department of Chemistry

Dr. Patrick E. Fleming

Department of Chemistry 


\title{
ABSTRACT \\ SYNTHESIS AND EVALUATION OF SILICA HYDRIDE-BASED FLUORINATED STATIONARY PHASES
}

\author{
by Kavita Vipool Prajapati
}

Silica-based fluorinated bonded stationary phases have shown enhanced selectivity with altered elution orders for molecules differing in hydrophobicity and hydrophilicity in comparison with $\mathrm{C} 8$ and $\mathrm{C} 18$ reversed phase columns. Hence, two novel silica hydride-based fluorinated bonded phases have been synthesized using a hydrosilation procedure to exploit fluorine-based unique selectivity for polar basic metabolites. Bonded moieties have been characterized by elemental and spectral analyses. Silica hydride-based aqueous normal phase (ANP) chromatography has retention behavior similar to normal phase chromatography, except for the use of water as a part of the binary solvent ( $>60 \%$ acetonitrile : water). In ANP, a higher percentile of nonpolar mobile phase shows increased retention for acids and bases, and nonpolar solutes can also be retained as in reversed phase chromatography (RPC). The synergistic effects of fluorinated phases' altered selectivity and aqueous normal phase retentivity have been explored for small polar metabolites using high performance liquid chromatography (HPLC) coupled with several detectors. Hydride-based fluorinated stationary phases showed good stability and remarkable reproducibility in retention time with $\%$ RSD $<1$. 


\section{ACKNOWLEDGEMENTS}

I would like to give my sincere thanks to Dr. Joseph J. Pesek for being my research advisor and providing me an opportunity to work on this project. I am grateful for his valuable feedback during the course of the research work. I truly appreciate his guidance in writing my thesis and his insightful comments in evaluating my thesis. I am also grateful to Dr. Maria Matyska Pesek for teaching me new instruments and techniques during the period of my research. I would like to take this opportunity to thank my committee members, Dr. Roger Terrill and Dr. Patrick E. Fleming, for helping me from time to time during my research work and for evaluating my thesis. I cannot thank enough to the chemistry department team members: Mr. Michael Stephens, the lead technician, and Mr. Craig Wood, the equipment technician, for providing me with their assistance for the lab instruments.

Finally, I am heartily thankful to my husband, Vipool Prajapati, for his endless support and understanding throughout my research work. I am deeply thankful to my little warriors, Rushil Prajapati (5) and Samarth Prajapati (2), for providing me all their

mommy-and-me time to finish up my research work and thesis. I am immensely grateful to our parents for their encouragement and support in focusing towards the goals of my research. 


\section{TABLE OF CONTENTS}

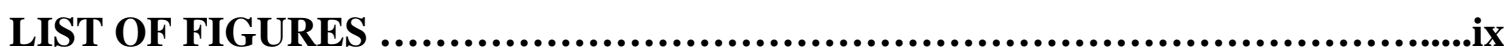

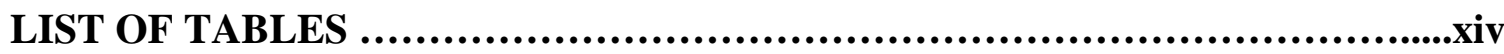

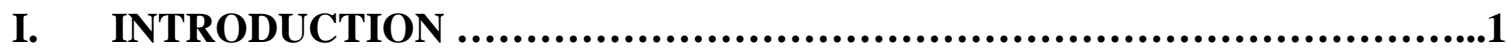

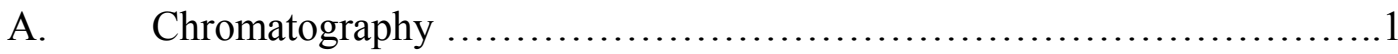

B. High Performance Liquid Chromatography (HPLC)..................................2

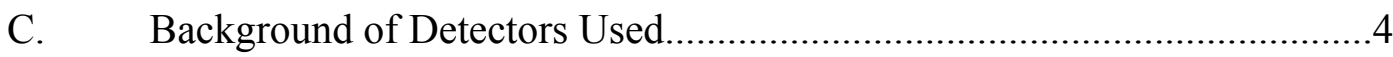

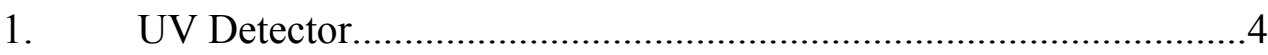

2. Evaporative Light Scattering Detector (ELSD)...............................

3. Mass Spectrometric Detector (LC-MS) ...........................................9

D. Aqueous Normal Phase Chromatography................................................12

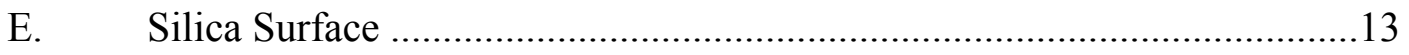

F. Surface Modification................................................ 14

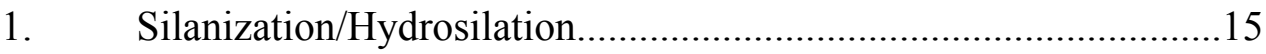

G. Fluorinated Stationary Phases.................................... 16

H. Characterization Techniques.....................................21

1. Diffuse Reflectance Infrared Fourier Transform Spectroscopy

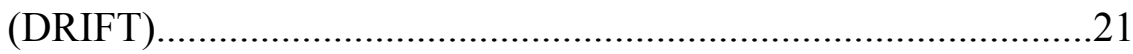

2. Carbon Elemental Analysis.........................................................22

3. Nuclear Magnetic Resonance Spectroscopy (NMR)......................23

I. Types of Samples Used in the Evaluation of Columns....................24

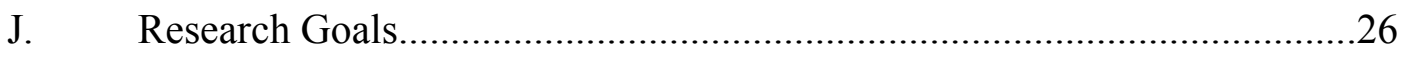




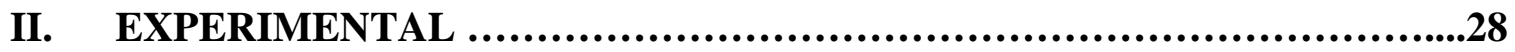

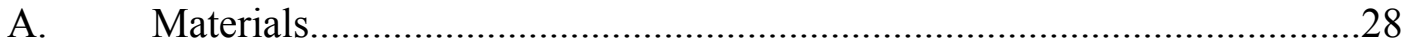

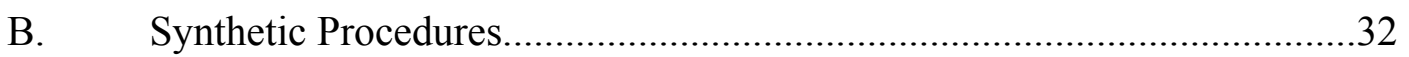

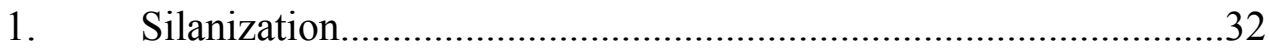

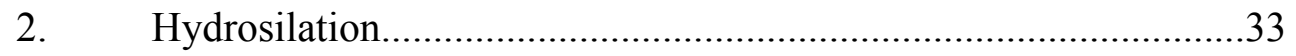

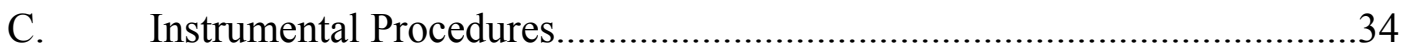

1. Diffuse Reflectance Infrared Fourier Transform Spectroscopy

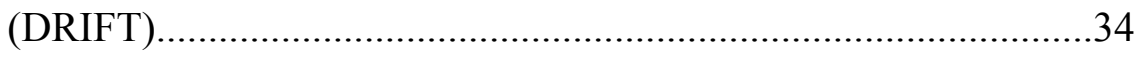

2. Carbon Elemental Analysis................................................................

3. Nuclear Magnetic Resonance Spectroscopy (NMR).......................35

4. High Performance Liquid Chromatography (HPLC).......................36

\section{RESULTS AND DISCUSSION .................................................39}

A. DRIFT Spectroscopic Characterization...................................................

B. Carbon Elemental Analysis Predictions...................................................43

C. Nuclear Magnetic Resonance Spectroscopic Confirmations.......................44

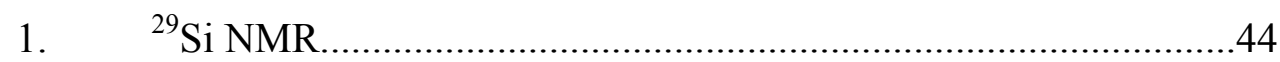

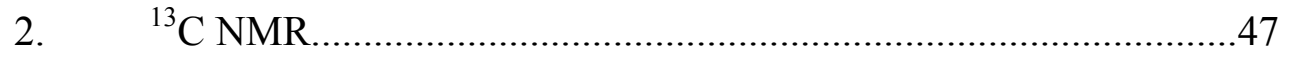

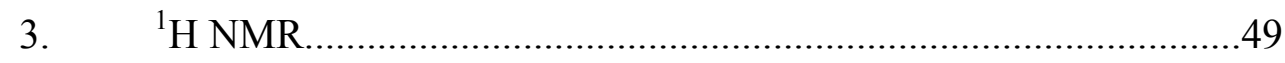

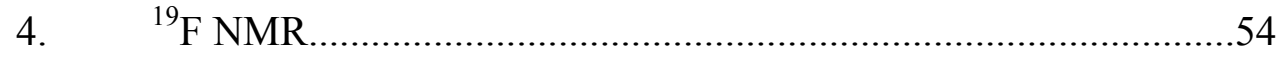

D. HPLC Chromatographic Evaluation of Columns.......................................59

1. Retention Studies Using ELSD Detection.........................................59

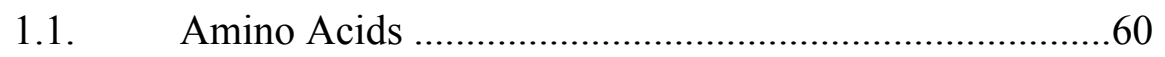


1.1.1. Reproducibility Study (\% RSD)..........................70

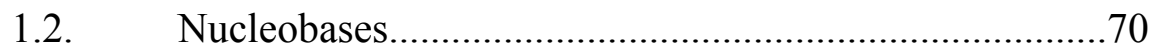

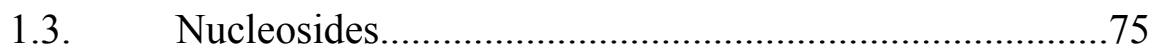

1.4. Ergogenic Acids..............................................................

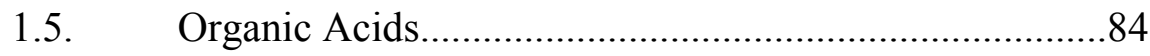

2. $\quad$ Retention Studies Using UV Detection..........................................86

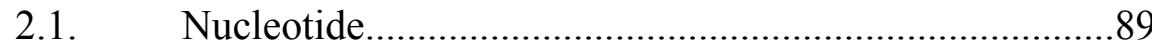

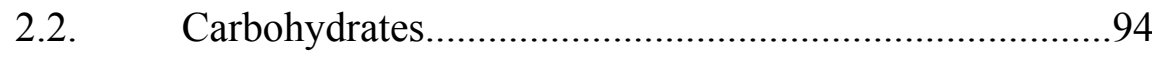

3. Retention Studies Using LC-MS Detection...................................100

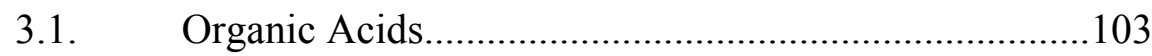

3.2. Carbohydrates.............................................................113

3.2.1. Monosaccharides................................................113

3.2.2. Disaccharides..............................................122

3.3. Amino acids.................................................................

4. Verification of Retention Data Using Different Detectors............140

4.1. Amino acids on ELSD and LCMS.................................140

4.2. Monosaccharide and disaccharides on UV and

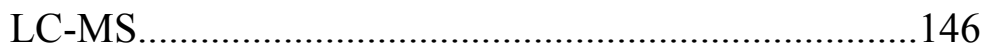

5. Acetone Based ANP Retention.....................................................150

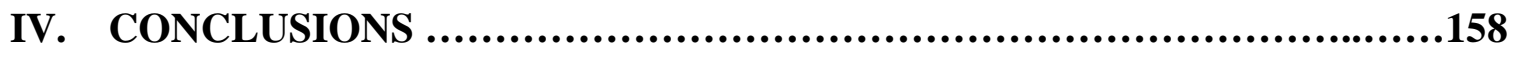

REFERENCES ......................................................162 


\section{LIST OF FIGURES}

FIGURE

PAGE

1. Schematic diagram of Evaporative Light Scattering Detector.................................6

2. Schematic diagram of Thermo-Split technology. Nebulization chamber walls A. heated for easy to evaporate mobile phases B. cooled down for hard to evaporate

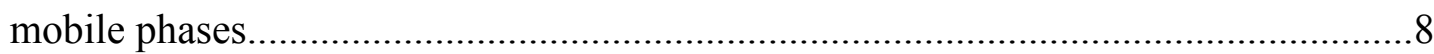

3. Basic design of mass spectrometer detector interfaced with liquid chromatography

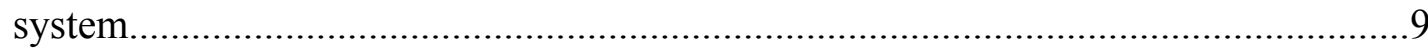

4. Schematic diagram of silanization and hydrosilation syntheses.............................15

5. (A) Phenyl- and (B) Alkyl- based fluorinated stationary phases............................18

6. Synthesis of perfluoro hydride- based stationary phases using silanization and

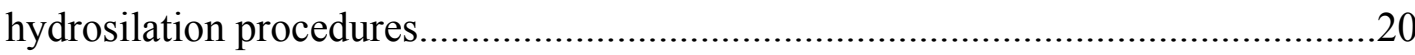

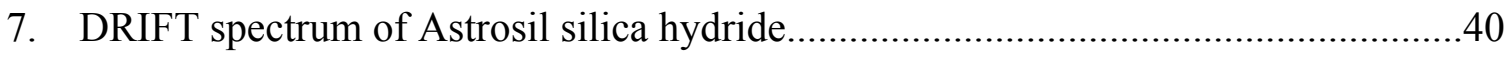

8. DRIFT spectrum of tridecafluoro-1-octene silica hydride...................................41

9. DRIFT spectrum of heptadecafluoro-1-decene silica hydride...............................42

10. Silicon-29 CP-MAS NMR spectrum of TDF C8 ...............................................45

11. Silicon-29 CP-MAS NMR spectrum of HDF C10 ........................................46

12. Carbon-13 CP-MAS NMR spectrum of TDF C8..........................................47

13. Carbon-13 CP-MAS NMR spectrum of HDF C10..............................................48

14. Proton-1 slurry state NMR spectra of Astrosil silica and silica hydride...................50

15. Proton-1 - slurry state NMR spectra of TDF C8 and silica hydride........................52

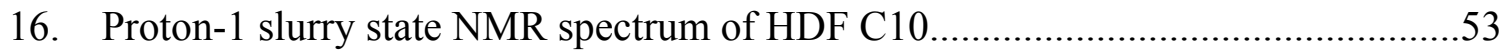


17. Fluorine-19 solution state NMR of heptadecafluoro-1-decene and tridecafluoro-

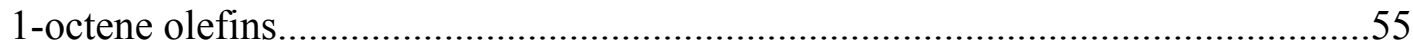

18. Fluorine-19 slurry state NMR of Astrosil silica hydride............................................56

19. Fluorine-19 slurry state NMR of TDF C8

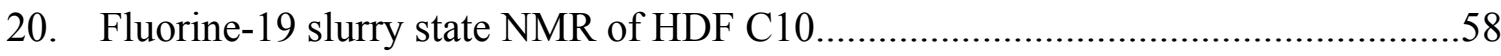

21. Retention map of amino acids on the HDF C10 column using ELSD.....................63

22. Retention map of amino acids on the TDF C8 column using ELSD........................65

23. Comparison of retention maps of tyrosine and phenylalanine on the TDF C8

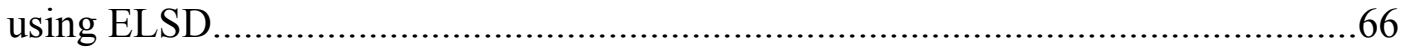

24a. Comparison of retention maps of arginine analyzed on two columns......................67

24b. Comparison of retention maps of histidine analyzed on two columns.....................68

24c. Comparison of retention maps of tyrosine analyzed on two columns......................68

24d. Comparison of retention maps of phenylalanine analyzed on two columns...........69

25. Retention map of nucleobases on the TDF C8 column using ELSD........................73

26. Retention map of nucleobases on the HDF C10 column using ELSD......................75

27. Retention map of nucleosides on the TDF C8 column using ELSD ........................77

28. Retention map of nucleosides on the HDF C10 column using ELSD......................79

29. Retention map of ergogenic metabolites on the TDF C8 column using ELSD........81

30. Retention map of ergogenic metabolites on the HDF C10 column using ELSD.....83

31. Comparison of retention maps of creatinine on the TDF C8 and HDF C10

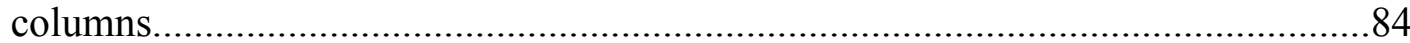

32. ANP retention of fumaric acid and citric acid with $0.1 \% \mathrm{FA}$ additive on the 
HDF C10 column and TDF C8 column respectively using ELSD........................86

33. ANP retention of ATP on the HDF C10 using UV detection................................92

34. Comparison of ANP retention of ATP on the HDF C10 and TDF C8 columns using UV detection

35. Retention maps of glucose and fructose with $0.1 \%$ FA and $15 \mathrm{mM}$ ammonium formate additives using UV detection.

36. Comparison of retention maps of glucose and fructose on the TDF C8 and HDF C10 columns using UV detection

37. ESI mass spectra of maleic acid, fumaric acid, succinic acid, and citric acid as $[\mathrm{M}-1]^{-1}$ molecular ion recorded using negative polarity mode.

38. Succinic acid: total ion current, ESI (-) mass spectrum of molecular ion $(\mathrm{M}-1)^{-1}=117 \mathrm{amu}$, and SIM chromatogram on TDF C8 for 50:50 acetonitrile: water (at $\mathrm{pH} \sim 6)$. .106

39. Retention map of organic acids on the TDF C8 column using LCMS. .108

40. Retention map of organic acids on the HDF C10 column using LCMS.

41. Retention map of isobaric fumaric acid and maleic acid on the TDF C8 column using LCMS.

42. Comparison of retention maps of citric acid on the TDF C8 and HDF C10 columns

43. Glucose: total ion current, ESI $(+)$ mass spectrum of sodium adduct $(\mathrm{M}+\mathrm{Na})^{+1}$ $=203.1 \mathrm{amu}$, and SIM chromatogram on TDF C8 for 95:05 acetonitrile:water (at $\mathrm{pH} \sim 6$ ) 


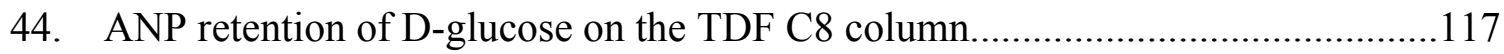

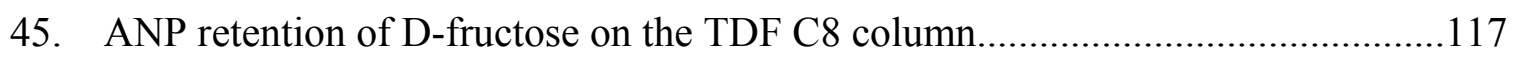

46. ANP retention of D-ribose on the TDF C8 column........................................... 118

47. Retention map of monosaccharides on the TDF C8 column...............................119

48. Retention map of monosaccharides on the HDF C10 column...........................121

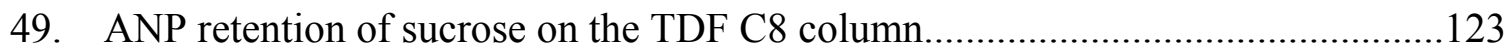

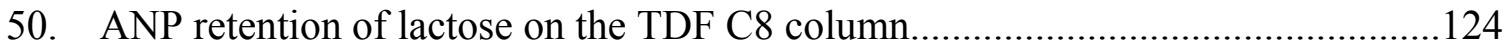

51. ANP retention of maltose on the TDF C8 column...........................................125

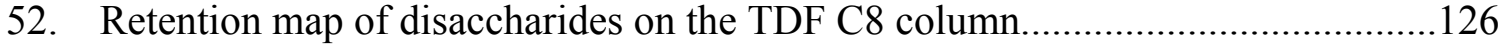

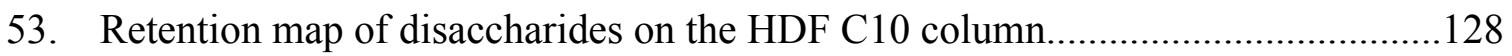

54. Aqueous normal phase chromatographic retention of sucrose as the acetonitrile part of mobile phase increases from $50 \%$ to $95 \%$ on the HDF C10 column.........129

55. Aqueous normal phase chromatographic retention of trehalose as the acetonitrile part of mobile phase increases from $50 \%$ to $95 \%$ on the TDF C 8 column..........133

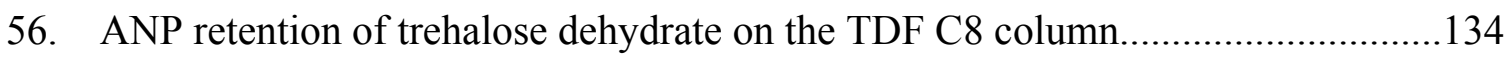

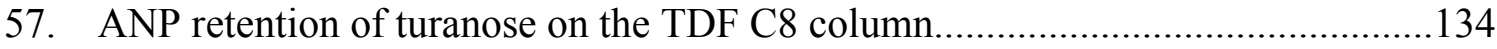

58. Reversed phase retention of nonpolar cellobiose octaacetate on the TDF C8 column in the form of SIM of $(\mathrm{M}+\mathrm{Na})^{+}$adduct $=701.2$ amu using ESI $(+)$ mode MS analysis

59. Retention map of polar and nonpolar disaccharides on the TDF C8 column using isocratic run.

60. Retention map of polar and nonpolar disaccharides on the HDF C10 column 
using isocratic run.

61. Verification of the retention maps of amino acids on the TDF C8 column using ELSD and MS detectors: $0.4 \mathrm{~mL} / \mathrm{min}$ flow rate was used in HPLC-MS analysis and $0.5 \mathrm{~mL} / \mathrm{min}$ flow rate was used in HPLC/ELSD analysis

62. Verification of the retention maps of amino acids on the HDF C10 column using ELSD and MS detectors: $0.4 \mathrm{~mL} / \mathrm{min}$ flow rate was used in HPLC-MS analysis and $0.5 \mathrm{~mL} / \mathrm{min}$ flow rate was used in HPLC/ELSD analysis

63. Verifying ANP retention of monosaccharide on UV by LCMS

64. Verifying ANP retention of disaccharides on UV by LCMS.

65. Acetone based ANP retention of organic acids on the TDF C8 column using $\mathrm{MS}$

66. Acetone based ANP behavior of fumaric acid and RP behavior of cellobiose octaacetate on the same column TDF C8.

67. Comparable acetonitrile- based and acetone- based ANP retention maps of organic acids on the TDF C8 column 


\section{LIST OF TABLES}

TABLE

PAGE

1. Chemicals used in the synthesis of the stationary phases.....................................28

2. Chemicals used in the preparation of the mobile phases........................................29

3. Samples analyzed for the aqueous normal phase HPLC retention studies................29

4. Surface coverage values of the bonded organic moieties.....................................43

5. Retention times of amino acids on the HDF C10 column using ELSD....................62

6. Retention times of amino acids on the TDF C8 column using ELSD......................64

7. Reproducibility for 10 injections of two amino acids using ELSD........................71

8. Retention times of nucleobases on the TDF C8 column using ELSD.......................72

9. Retention times of nucleobases on the HDF C10 column using ELSD....................74

10. Retention times of nucleosides on the TDF C8 column using ELSD.....................76

11. Retention times of nucleosides on the HDF C10 column using ELSD...................78

12. Retention times of ergogenic metabolites on the TDF C8 column using ELSD......80

13. Retention times of ergogenic metabolites on the HDF C10 column using ELSD....82

14. Retention times of organic acids for $0.1 \%$ FA additive using ELSD.....................85

15. Retention times of ATP on both TDF C8 and HDF C10 columns using UV

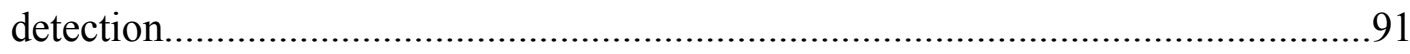

16. Retention times of glucose and fructose on the TDF C 8 column with $0.1 \%$ FA and $15 \mathrm{mM}$ ammonium formate additives using UV detection.............................95

17. Retention times of glucose and fructose on both the TDF C8 and HDF C10 columns with $15 \mathrm{mM}$ ammonium formate additive using UV detection. 
18. Retention times of disaccharides on the TDF C8 column using UV detection

19. Molecular weights and molecular ions monitored for ANP retention study of

(A) organic acids (B) amino acids, and (C) carbohydrates

20. Retention times of organic acids on the TDF C8 column using LCMS

21. Retention times of organic acids on the HDF C10 column using LCMS

22. Retention times of monosaccharides on the TDF C8 column using LCMS

23. Retention times of monosaccharides on the HDF C10 column using LCMS.

24. Retention times of disaccharides on the TDF C8 column using LCMS

25. Retention times of disaccharides on the HDF C10 column using LCMS.

26. Retention times of polar and nonpolar disaccharides on the TDF C8 column using LCMS

27. Retention times of polar and nonpolar disaccharides on the HDF C10 column using LCMS.

28. Retention times of amino acids on the TDF C8 column using LCMS

29. Retention times of amino acids on the HDF C10 column using LCMS

30. Retention times of monosaccharide on the TDF C8 column using LCMC and UV

31. Retention times of disaccharides on the TDF C8 column using LCMS and UV ...148

32. Retention times of polar and nonpolar solutes using acetone as a binary organic mobile phase solvent on the TDF C8 column using LCMS.

33. Comparison of acetonitrile- and acetone- based aqueous normal phase retention of organic acids on the TDF C8 column using LCMS. 


\section{INTRODUCTION}

\section{A. Chromatography}

Chromatography encompasses very important scientific methods to accomplish separation of closely related components of complex mixtures. Its discovery around 1903 is credited to a Russian scientist Mikhail Twsett, for his work in separating plant pigments on a chalk column using ether. Separated components appeared as color bands on the column that justifies the meaning of chromatography as a process of "color writing" in Greek. Since this initial work, large numbers of systems and techniques have been developed to make chromatography a powerful tool for analytical separations $[1,2]$.

The basic concept of chromatography involves the use of a stationary phase and a mobile phase. The sample to be separated is transported in the mobile phase which may be a gas, a liquid, or a supercritical fluid. The stationary phase may be a support material fixed in the column. Once transported, the sample components distribute themselves between the stationary phase and the mobile phase with varying degrees of interactions. The components attracted by the stationary phase, retain more and move slowly with the flow of mobile phase, whereas, those with lower interaction move faster eluting earlier. Hence, this difference in mobility separates the components of sample into discrete bands or zones that can be confirmed for individual identity.

The stationary phase is either held in a narrow tube (column) as in column chromatography or is supported on a flat plate as in planar chromatography, and the mobile phase is forced through column in the direction of gravity or it migrates by capillary action against gravity respectively. The majority of applications incorporate 
column chromatography. On the basis of the physical state of the mobile phases being used, chromatography can be further classified as gas chromatography (GC), liquid chromatography (LC), and supercritical-fluid chromatography (SFC). Only liquid chromatography can be performed on column as well as on flat surfaces [1].

In liquid chromatography, the mobile phase is a liquid that is in contact with a solid or liquid stationary phase. The advances in column technology and fabrication of packing materials demanded use of reliable pumps to produce high pressure flow rates for better and faster separation in liquid chromatography. Therefore, the term HPLC has been appropriately introduced for high pressure or high performance liquid chromatography that uses sophisticated instrumentation for achieving higher performance in separation analysis [1].

\section{B. High Performance Liquid Chromatography (HPLC)}

High performance liquid chromatography specifies separation analysis that uses a column containing small diameter particles $(\leq 10 \mu \mathrm{m})$, a pump capable of pushing solvent through such columns and a detector that can measure the compounds separated with good sensitivity. In industries like biotechnology and pharmaceuticals, HPLC has been recognized as a powerful tool for the purpose of identification, characterization, purification, and separation of biomolecules [2].

Modern HPLC instruments include a solvent delivery system, a sample introduction system, a separation system (column), and a detection system. The primary component of the solvent delivery system is the pump; however, it also consists of solvent reservoirs, degassers, mixers, gradient formers, and pulse dampers. The dissolved gases 
like oxygen and nitrogen may interfere in the analysis if not removed from the mobile phases. Procedures like helium sparging and online degassing can remedy this problem. A constant composition of one or more solvents (isocratic) can be used to analyze the samples, and one can also achieve higher separation efficiency using gradients run with varying mobile phase composition per time. The pumping system can generate constant pressure up to $6000 \mathrm{psi}$ to adjust the mobile phase flow rates ranging from 0.1 to 10 $\mathrm{mL} / \mathrm{min}$. For injecting the sample, a manual injector or an autosampler can be used. The manual ones are loop injectors that deliver the sample into the column in a smooth and continuous form by a single switch valve. On the other hand, autosamplers use an automated sample injector to introduce varying sample volumes as frequently as several per minute from the same vial.

Once the sample is injected into the column, the constant flow of mobile phase pushes the sample down the column to separate its components between itself and the stationary phase. The columns are made up of a stainless steel body or a polymeric tube containing $1.5-10 \mu \mathrm{m}$ sized silica particles of varying porosity. Since the characteristics of these particles play an important role in HPLC based separation, the column is considered the "heart" of the HPLC separation system [1, 2].

Finally, the detection system attached to the column produces an electrical signal proportional to the concentration of the separated components. This signal is plotted as a chromatogram measuring concentration against elution time or elution volume. An ideal detection system should provide adequate sensitivity for tracking a particular component. Not only better sensitivity, but lower noise, faster response, lower dead 
volume and lower temperature sensitivities are also some of the important factors that need to be evaluated when deciding on HPLC detectors. Since different detectors operate on different physical or chemical principles, further classification can be made with respect to its ability to identify the analyte molecules. For example, ultraviolet-visible, fluorescence, and radioactive detectors are selective detectors as they respond to specific analytes only, whereas refractive index (RI) and mass spectrometers (MS) are universal detectors detecting virtually every type of analyte $[1,2]$.

\section{Background of Detectors Used}

\section{Ultraviolet (UV) Detector}

UV detectors are the most commonly used HPLC detectors; they quantify the compounds on the basis of Beer's Law: the absorbance of an analyte, at a particular UV wavelength, is directly proportional to its concentration. For selecting a characteristic UV wavelength, the detector is constructed to use different filters or a monochromator. More sophisticated detectors allow scanning of multiple UV wavelengths with multiple filters, a prism or a grating. Along with its apparent advantages of high sensitivity and easy operation; it is important to note that the analyte must absorb in a UV wavelength range. Many molecules do not carry the chromophores required for making them UVactive and that limits the applicability of UV detection system $[1,2]$.

\section{Evaporative Light Scattering Detector (ELSD)}

The evaporative light scattering detector is a near universal HPLC detector as it can detect almost any analyte that is less volatile than the mobile phase. Besides liquid chromatography, its compatibility with countercurrent chromatography and supercritical 
fluid chromatography has also been reported. ELSD was first reported in 1966 by Union Carbide's Australian research lab. Later on, it became available commercially around $1980[3]$.

Due to its potential advantages over other detectors, ELSD has become a supplement to or substitute for many other HPLC detectors. Unlike UV, it can detect the analytes with or without chromophores, and will not give any negative peaks. The gradient profile will be stable during the change of mobile phase composition as it does not respond to solvent concentration. Consequently, it does not give solvent front peaks at the void volume unlike UV and refractive index (RI) detectors making it a superior choice for detection of early eluting analytes. In contrast to a mass spectrometer (MS) detector, it is less complex and has a low cost of operation which is a plus for its use in method development analysis. The only drawback to point out would be its destructive mode of operation; therefore, the sample can not be collected at the end of the analysis for preparative scale applications [4].

Provided that the analyte is less volatile than mobile phase, any unknown analyte can be tested using following three successive steps of nebulization, evaporation and detection. The schematic representation of ELSD's operation is outlined in the Figure 1.

Nebulization transforms the HPLC effluents into an aerosol cloud that is made up of fine droplets. The constant flow of inert gas helps to form uniform droplets in the aerosol cloud. This uniformity of droplets ensures sensitivity and reproducibility of the analysis. Afterwards, the aerosol cloud is carried into the heated evaporation chamber (drift tube) by the carrier gas where the mobile phase evaporates leaving behind the droplets or 
particles of pure analyte. The temperature of the drift tube has to be selected carefully for the purpose of the solvent evaporation. When the analyte cloud enters the detection cell, it passes through the beam of laser light where it scatters some of the light. The scattered light is detected by the silicon photodiode which is located at an appropriate angle. The output signal is proportional to the size and the size distribution of the incoming analytes $[3,4]$.

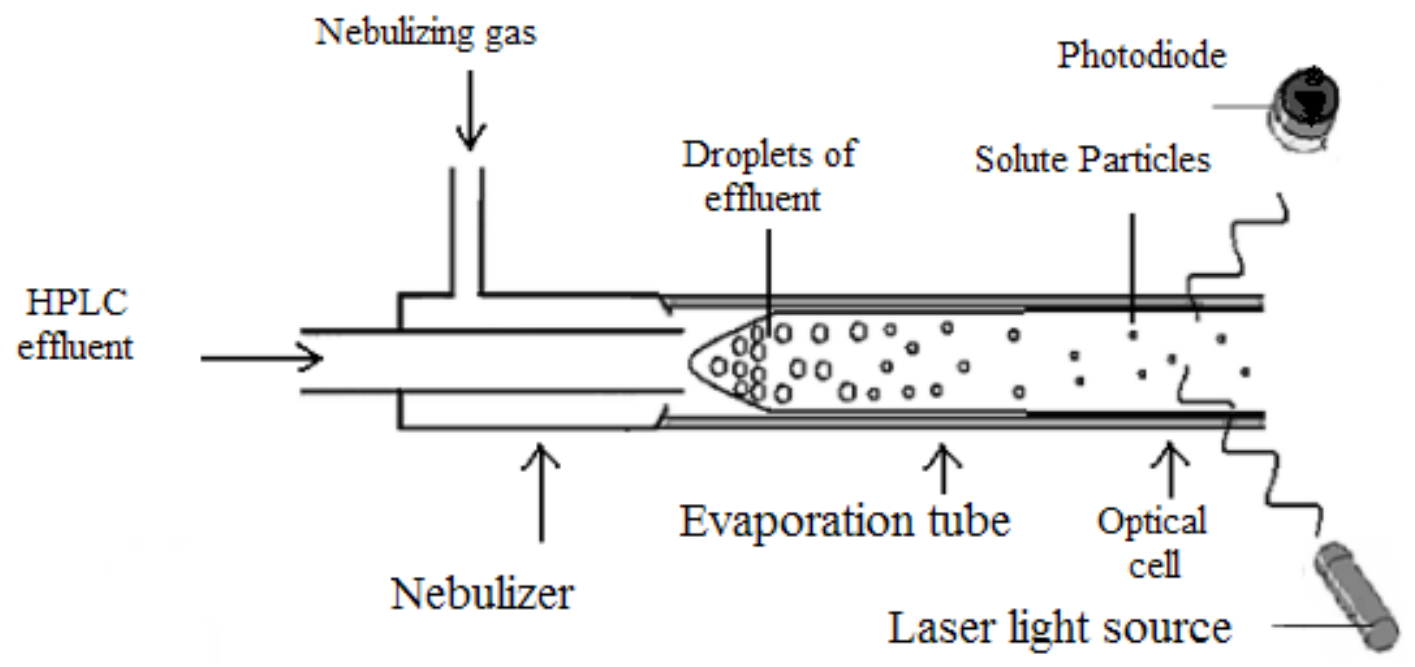

Figure 1. Schematic diagram of Evaporative Light Scattering Detector.

(Adapted from [3], Megoulas, N. C.; Koupparis, M. A. Critical Reviews in Analytical Chemistry, 35, 301-316, 2005).

It is worthwhile to mention briefly that the ELSD detection is mainly based on elastic light scattering in which the scattered light possesses the same frequency as the incident light. The elastic scattering phenomena is observed in the form of Rayleigh, Debye, and 
Mie -scattering. In general, these scattering phenomena depend on different interactions between the light and the size, shape, and surface properties of the particles [3].

In ELSD, the light scattering is proportional to the size of the particle rather than its concentration. Hence, the correlation between the chromatographic peak area (A) and the analytes' mass (m) can be presented as described in the following equation.

$$
\log \mathrm{A}=\mathrm{b} \log \mathrm{m}+\log \mathrm{a}
$$

where $a$ and $b$ are coefficients depending on the ELSD's instrumentation parameters and its operation variables $[2,3]$.

One more interesting feature of ELSD is its ability to split the aerosol cloud. When it is necessary to generate higher HPLC flow rates, ELSD needs a way to divert a part of the aerosol cloud to the waste. Introduction of a sharp turn or a plate impactor in the nebulization chamber have shown the splitting of the unnecessary amount of aerosol cloud. However, SofTA Corporation's new patent pending thermo-split technology has resulted in improved solvent splitting technology that can handle lower, moderate, and higher flow rates of various mobile phases.

As shown in the Figure 2, a thermo-split based nebulization chamber (spray chamber) has walls that can be heated or cooled down to assist gentle temperature variations. When an easily evaporated mobile phase or lower flow rates are encountered, these walls are maintained at ambient or slightly elevated temperatures. As aerosol traverses the spray chamber, its partial evaporation which has already been started, leaves behind a lower amount of aerosol cloud that can easily negotiate the spray chamber band. When 
hard to evaporate mobile phases or higher flow rates are used, the walls are cooled down to condense the extra load of aerosol down the drain. Thus, heated walls or cooled walls of the spray chamber can smoothly divert the unnecessary aerosol cloud to achieve optimum selectivity and sensitivity [4].

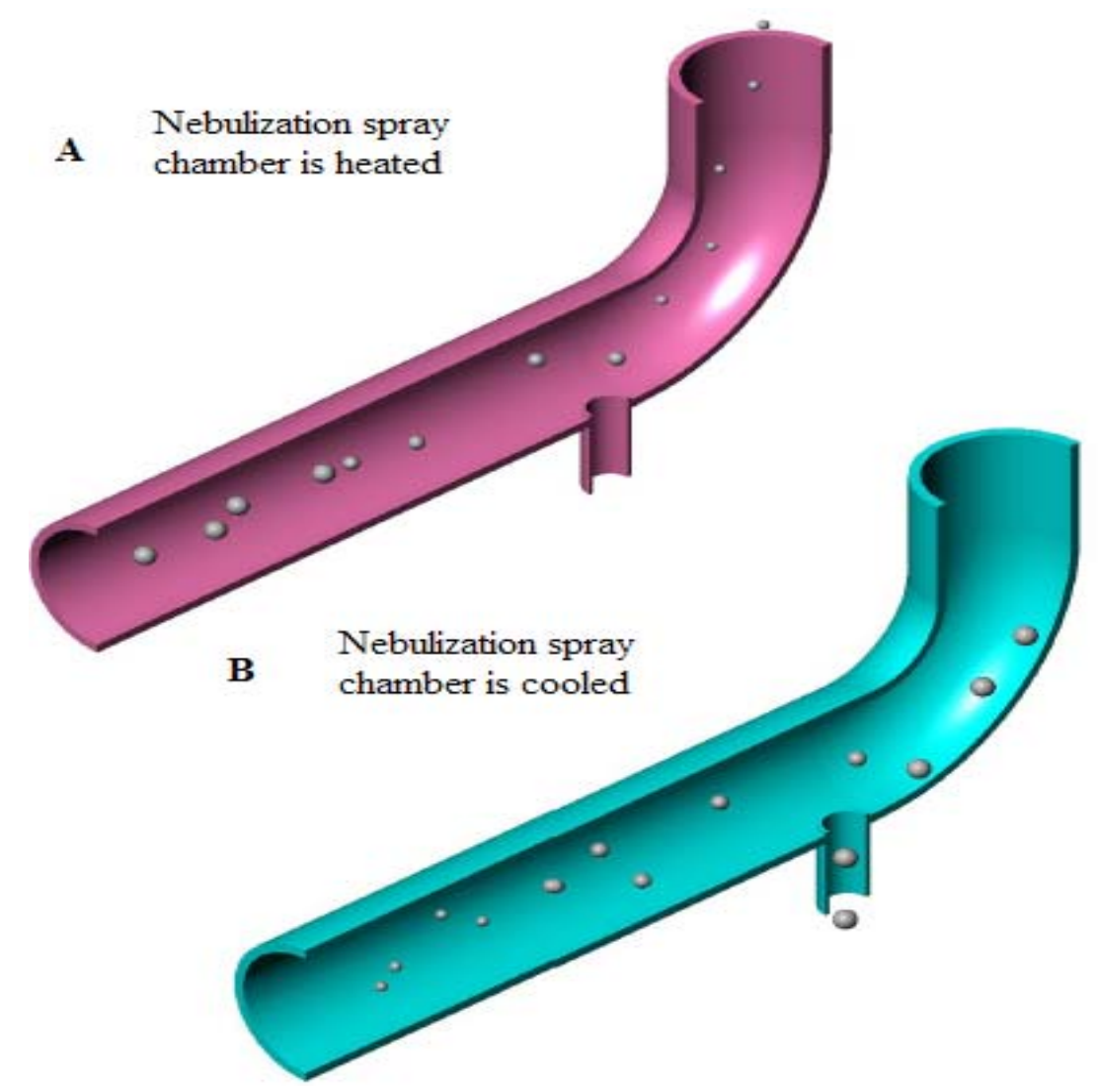

Figure 2. Schematic diagram of Thermo-Split technology. Nebulization chamber walls A. heated for easy to evaporate mobile phases B. cooled down for hard to evaporate mobile phases. (Adapted from [4]: Model 400 ELSD manual, v. 1.2, 2006, http://www.softacorportion.com, accessed November 2009).

The effluents eluting from the column require splitting into tiny fractions for the 
purpose of detection by mass spectrometer (MS) as well. However, newly available interface technologies have made LC-MS coupling viable [1].

\section{Mass Spectrometric Detector (LC-MS)}

Mass spectrometry has been recognized as an ideal detector for its universality and unique selectivity. Its ability to identify each of the analyte species with high resolution can be credited to the solvent-assisted ionization type of interface with the LC system. Examples include thermospray, electrospray, and atmospheric chemical ionization interfaces. This general set up removes the bulk of the mobile phase from the sample, and transforms the analyte molecules into gaseous ions. These gaseous ions are an appropriate form of the analytes to be introduced into the high vacuum mass analyzers [2]. Figure 3 represents a basic diagram of an MS detector coupled with an HPLC system via electrospray (ESI) or atmospheric pressure chemical ionization (APCI) interfaces.

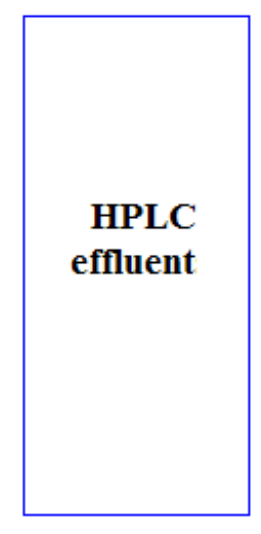

Sample Introduction

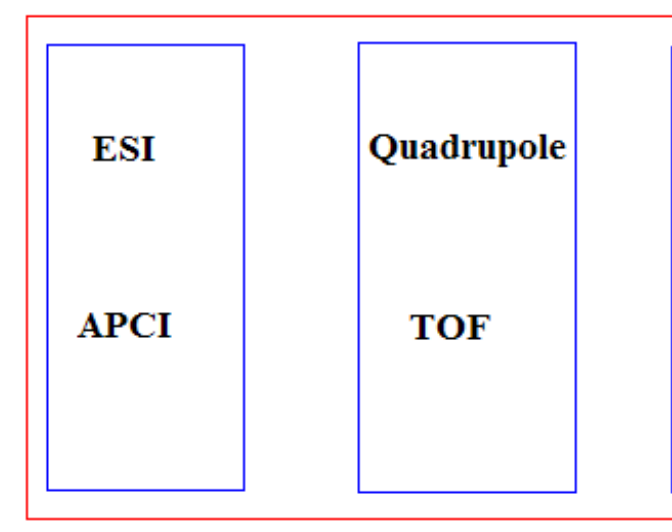

Ionization

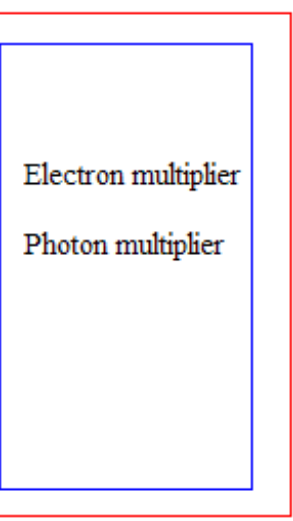

Detection

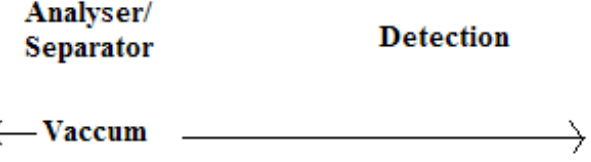

Figure 3. Basic design of mass spectrometer detector interfaced with liquid chromatography system. 
Compared to other ionization sources, an electrospray probe has been a popular choice of ionization in biopolymer analysis. Since it is based on the soft ionization principle, biomolecules can be detected in the form of singly charged molecular ions at atmospheric pressure. The combination of nebulization and ionization in ESI results in the attachment or removal of one or more protons, from the solvents to the analytes thus forming the molecular ions. The inert carrier gas draws these molecular ions to the mass analyzer for the separation based on their mass to charge $(\mathrm{m} / \mathrm{z})$ ratio [2]. The quadrupole mass analyzer is a commonly used ion separator because of its ruggedness and lower cost compared to the time of flight (TOF).

Generally, the quadruple mass analyzer consists of four cylindrical rods mounted in a ceramic collar, forming an ion path. By choosing an appropriate ratio of radio frequency and DC voltage applied to these rods, desired molecular ions can be focused to pass through the vacuumed quadrupoles towards a photomultiplier detector. These detected molecular ions are plotted as ion counts versus ion mass in the form of mass spectra. The sensitivity of an analyte ion can be plotted against the function of time in the form of total ion current (TIC) or single ion monitoring (SIM) in the case of a specific separation study [5].

With these choices of detectors, one can selectively analyze different types of molecules. Furthermore, the mechanistic understanding of the separation process is equally essential for adequate analysis. The definition of liquid chromatography can be further classified into adsorption chromatography and partition chromatography 
according to the mechanism involved in the separation process. The solute is retained on the basis of its distribution among two or more phases, which is defined as a partition, and its retention due to attractive forces between itself and solid surface is defined as an adsorption $[1,6]$.

The widely used partition chromatography can be classified into either liquid-liquid or bonded-phase chromatography depending on how the stationary phase is held on the support surface. Since the first mode has a major disadvantage of dissolution in the mobile phase over time, the bonded-phase has become a generally adapted approach. The success in the chemical bonding of different organic moieties (bonded-phases) onto the support material has opened the door for performing highly selective partition liquid chromatography [1].

Partition chromatography can be subcategorized into normal phase and reversedphase chromatography. Normal phase (NP) chromatography utilizes polar stationary phases like cyano, amino or triethyleneglycol bonded on bare silica or alumina, and nonpolar mobile phases like hexane or ether (with methylene chloride, propanol, or methanol as a modifier) to elute polar solutes. Conversely, reversed phase (RP) chromatography employs nonpolar stationary phases like octadecyl (C18/ODS), octyl (C8), butyl (C4), phenyl or biphenyl; and polar mobile phases like water (with methanol or acetonitrile as a modifier) to elute nonpolar solutes $[1,2]$. Normal phase chromatography retains polar molecules and reversed-phase chromatography retains nonpolar molecules. However, the majority of biomolecules and drugs possess multiple polar sites, and are hard to separate solely based on NP or RP chromatography. Two 
dimensional chromatography (one can use RP and NP chromatography successively) is one of the options to separate multiple polarity biomolecules. In HPLC, the use of extreme $\mathrm{pH}$ and higher temperature might be other solutions for the separation of these compounds. These steps are time consuming and damaging to HPLC instruments and the columns [2].

\section{Aqueous Normal Phase Chromatography (ANP)}

Aqueous normal phase chromatography is a novel separation mechanism introduced by Pesek et al. as a third type of chromatography that can retain both polar as well as nonpolar analytes on the same column [6]. Hence, aqueous normal phase chromatography can address the separation of multiple polarity biomolecules with a simpler approach.

Aqueous normal phase chromatography (ANP) can be defined as a normal phase chromatography that employs water (polar) as a part of binary solvent system. When the less polar part of the binary solvent (mainly acetonitrile) is increased to $60 \%$ or more, polar solutes like acids and bases show retention as in the normal phase mode. When the aqueous component increases, nonpolar molecules are retained as in the reversed-phase mode. This unique dual retention capability of ANP is observed due to silica-hydride or silica-hydride based stationary phases synthesized using silanization/hydrosilation procedures [6]. The ANP behavior of an individual solute is described as solute's characteristic retention map by plotting its retention time versus concentration of mobile phase composition. 
Because of the fact that the silica hydride based stationary phases are highly stable, a wide range of binary solvent compositions can be tried -from 100\% aqueous to $100 \%$ organic- to retain and separate particular polar-nonpolar pairs. When the hydride column has higher water, it operates in the RP mode; at high organic with some water the ANP mode is dominant; and pure organic mobile phases provide organic normal phase (ONP) retention. Interestingly, the RP and ANP modes are complimentary to each other due to the common binary solvent system, and therefore can operate on the same column in a single isocratic run [7]. As an addition to the nonpolar solvent based ANP retention, the use of additives like formic acid (FA), acetic acid, ammonium formate and ammonium acetate improves the retention of polar acids and bases. The use of a fairly low concentration of these additives has shown compatibility with MS detection and the pumping system of an HPLC instrument $[6,8]$. Aqueous normal phase chromatography is observed specially for the silica hydride-based materials. The synthesis and advantages of silica hydride-based support materials are illustrated as follows.

\section{E. Silica Surface}

Silica is the most widely used separation medium in column chromatography due to its ever evolving practical applications. Originally, silica particles with irregular shape and wide pore size distribution were used as a support material in normal phase chromatography. This type of silica based stationary phase showed limited reproducibility and flexibility towards the use of normal phase solvents, hence it has been replaced by an enhanced version. Newer fabrication technologies have produced mechanically stable silica particles with uniform diameters and specific pore sizes. This 
type of carefully manufactured silica results in spherical shaped particles with higher surface area, which can be packed uniformly in columns to yield better efficiency and higher resolution [9].

Because silica is a condensation product of silicic acid, its polymeric surface carries exposed siloxane linkages (Si-O-Si) and silanols $(\mathrm{Si}-\mathrm{OH})$ on the outer layer. These silanols are in the form of isolated, vicinal, and geminal -type of free hydroxyl groups acting as polar acidic sites. These sites can readily interact with polar basic solutes resulting in poor separation performance and $\mathrm{pH}$ instability of the column material. This matter becomes serious when silica-based HPLC stationary phases are utilized for RP and NP separations. To minimize the surface silanols, endcapping with small organic molecules has been reported. However, this process does not replace all silanols and the endcapped version of silica showed limited $\mathrm{pH}$ stability $[10,11]$. The other options available for chemical modification of silica surface are discussed in the following section.

\section{F. Surface Modification}

Surface modification of silica is a well studied area of surface chemistry that mainly involves the conversion of silanols into bonded organic moieties using esterification, chlorination/Grignard reactions, or organosilanization [12]. This research utilizes a unique silanization procedure that has been shown to remove almost $95 \%$ of the total silanols on the surface of silica into silica hydride which has unique separation capabilities. Hydrosilation of silica hydride material introduces desired organic moiety on to the surface. Such hydride surface-based stationary phases have not only overcome 
the limitations of the silanols but also have resulted in interesting aqueous normal phase separations [13].

\section{Silanization/Hydrosilation}

Silanization and hydrosilation are two successive step syntheses producing silica hydride based stationary phases that have shown hydrophobic, hydrophilic, ion-exchange, chiral, etc. types of separation capabilities. The scheme of silanization/hydrosilation is presented in Figure 4.

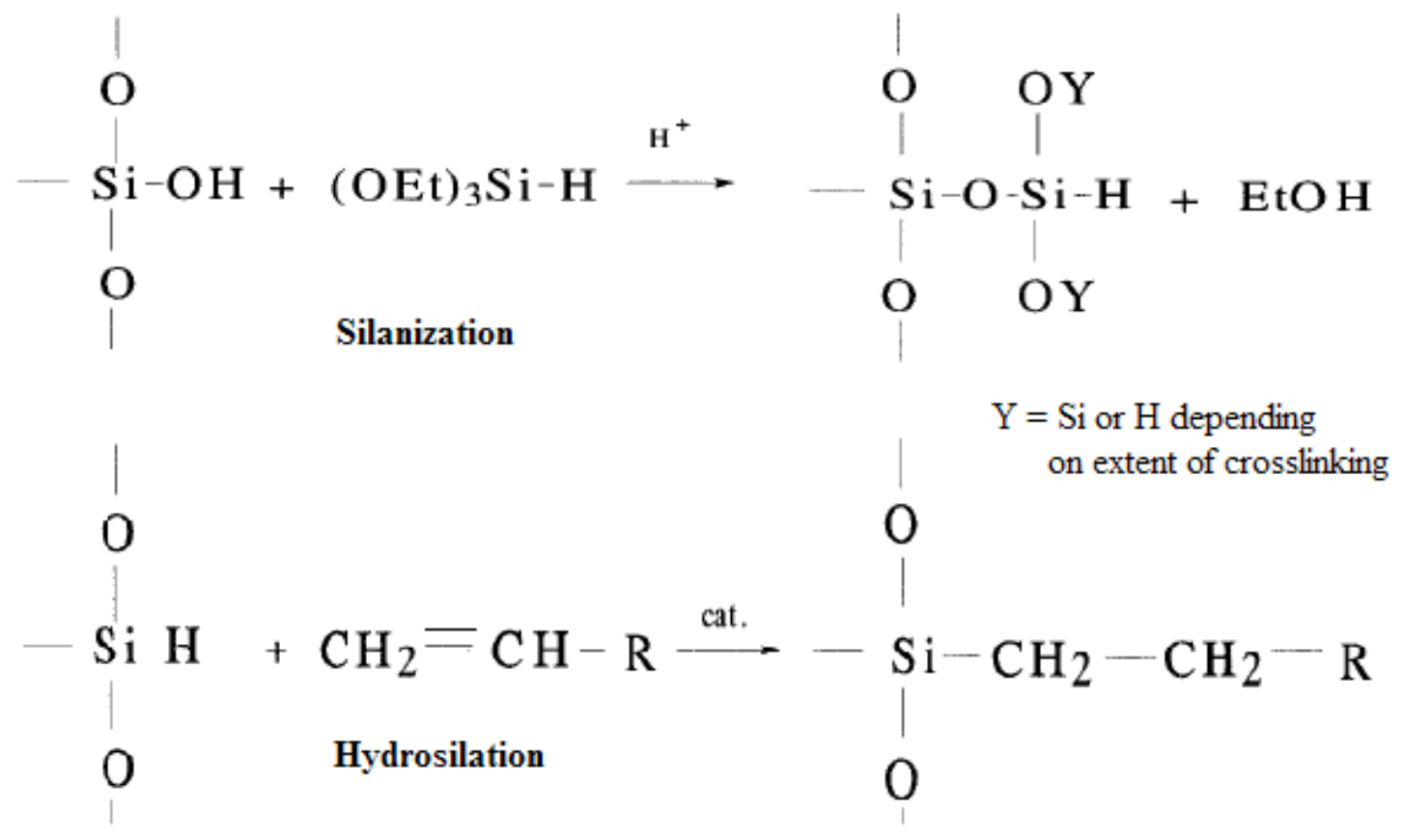

catalyst=hexachloroplatinic acid

Figure 4. Schematic diagram of silanization and hydrosilaiton syntheses.

In the silanization step, polar silanols are converted into hydrophobic silica hydride, under controlled reaction conditions of triethoxysilane (TES) concentration, water, acid, 
reaction temperature and time. This monolayer of newly formed silica hydride acts as a stable intermediate for the next step of hydrosilation.

In the second step of hydrosilation, the characteristic organic moiety can be attached in the form of a terminal olefin to the hydride intermediate by catalytic addition using hexachloroplatinic acid (Speier's catalyst) or free radical initiator. Other forms of organic moieties like non-terminal olefins, alkynes and cyano compounds have also been attached successfully to silica hydride intermediates. It is important to note that not all of the silica hydrides are being replaced by the organic moiety due to steric factors. The underlying silica surface still contains hydrides that influence the separation capabilities $[12,13]$. In this research, a perfluoro-octene and a perfluoro-decene (terminal olefins) were attached to silica hydride using the hydrosilation synthesis to make hydride based fluorinated stationary phases.

\section{G. Fluorinated Stationary Phases}

The unique chemical and physical properties of the fluorocarbons have been exploited in the development of a novel category of fluorinated LC stationary phases. The alkyl- and phenyl- based fluorinated and perfluorinated bonded moieties on the silica surface have shown selective and complimentary retention from the traditional C8 and C18 reversed phase materials. The majority of separation analyses of proteins and similar biomolecules with multiple polar sites require the use of complex mobile phase preparations and high $\mathrm{pH}$ conditions in the octadecylsilica (ODS) type reversed phase columns. However, replacing the $\mathrm{C}-\mathrm{H}$ portion of hydrocarbons in reversed phase 
materials with a C-F functionality as in fluorinated stationary phases introduces an apparent polar character that may enhance the retention of the biomolecules $[14,15]$.

In 1980, the de Galan group reported the very first preparation of alkyl fluorine bonded phases and their capability in separating the fluorinated compounds from their non-fluorinated analogs in LC [16]. Later on, Xindu and Carr reported the potential advantages of separating proteins on a $\mathrm{C}_{8} \mathrm{~F}_{17}$ fluorinated bonded phase using higher concentrations of the organic solvent (isopropanol) in the mobile phase [17]. They noted that the use of an organic solvent instead of the ion-paring additives for retention was less denaturing and easy to evaporate for preparative separations. They have also suggested that the fluorocarbons are less adsorptive than the hydrocarbonaceous materials; perhaps this fact explains the fluorinated phases' unique selectivity.

The separations of fluorinated and other halogenated solutes have shown higher selectivity on the fluorinated bonded phases than their non-fluorinated counter parts. The presence of fluorine-fluorine interaction has led to further studies using fluorous tagging on the solutes, and the use of the fluorinated alcohols as mobile phase modifiers to obtain desired separations of the biomolecules on fluorinated phases [16].

In general, the possible retention mechanisms that have been proposed are as follows: the alkyl- based fluorinated phases may possess dipole-dipole (dispersive) interactions in the case of fluoro/halo- or polar metabolite- types of solutes; the phenyl- based fluorinated phases may exhibit pi-pi interactions for the phenyl based solutes; even charge transfer and ion exchange mechanisms have been postulated for the ionic 


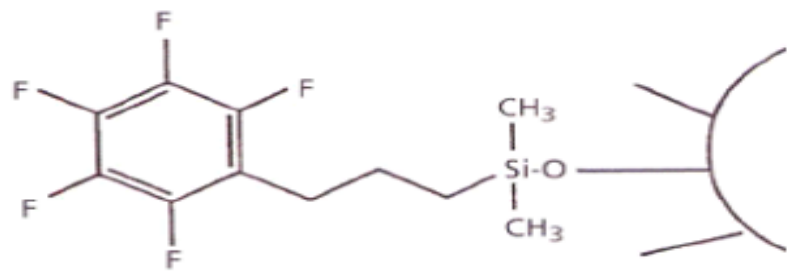

PFP (Penta Fluoro Phenyl)based fluorinated stationary phase.

(A)

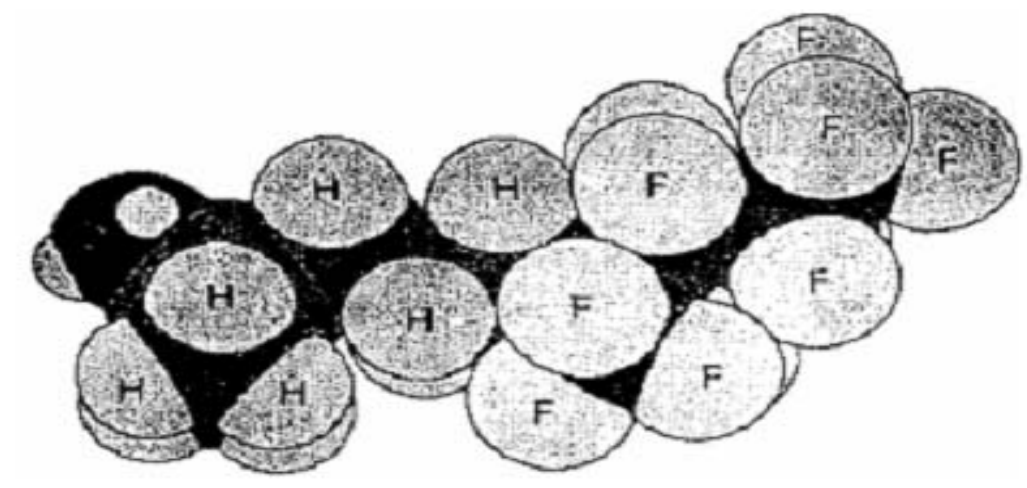

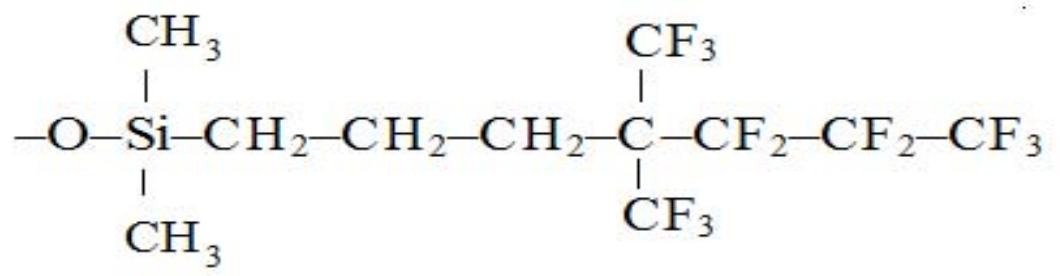

Chemical structure of alkyl based fluorinated bonded phase with its chemical model drawn using MM2 calculation.

(B)

Figure 5. (A) Phenyl- and (B) Alkyl- based fluorinated stationary phases.

(Adapted from reference 15, Przybyciel, M. LCGC Europe. 2006, 19, 1,

1-10; and reference 18, Jinno, K.; Nakamura, H. Chromatographia.

1994, 39, 5/6, 285-293, respectively). 
biological solutes. Many large and small, planar and nonplanar solute separations have been reported on the basis of the size and shape selectivity of the fluorinated phases [14, 16, and 18]. Hass and Kohler tried to explain the orthogonal selectivity of the fluorinated phases over respective hydrocarbon reversed phases [19]. Based on their proposal, the hydrocarbon chains have a high degree of association, exposing many adsorption sites for solute interaction, whereas the fluorinated moieties behave as isolated brushes for solute interactions. This prediction has provided an insightful theoretical point of view for further studies.

Various types of fluorinated phases have been reported: fluorinated or perfluorinated; alkyl- based or phenyl- based; straight chains or branched chains; on silica gels or on polymer supported fluorinated beads. When the fluorinated moiety is attached to silica, it requires the use of the spacers containing hydrogen e.g., methylene, ethylene or propylene. Silica based fluorinated stationary phases have exhibited high stability, and therefore high reproducibility in separation studies. Pentafluoro- phenyl (PFP) and branched $\mathrm{C}_{6} \mathrm{~F}_{13}$ (also called Fluofix) are some of the extensively studied silica based fluorinated phases with unique selectivity for polar as well as nonpolar solutes. Figure 5 shows the structures of two silica based fluorinated phases: (A) a phenyl- based fluorinated phase and (B) an alkyl-based fluorinated phase with its chemical model drawn using an MM2 calculation [15, 16, 18, and 19].

According to recent studies, the alternate selectivity of the fluorinated phases compared to the hydrocarbon reversed phases has become significantly evident for the retention of the polar and basic solutes when higher percentage of organic solvents like 
methanol and acetonitrile are used. Zhang [16] postulates that fluorinated phases could have different separation mechanisms depending on the type of analyte. For the polar basic solutes, the fluorinated phases show a "U-shape" retention profile (a plot of retention time versus percentage mobile phase composition) as the concentration of the organic content (e.g., methanol or acetonitrile) of the mobile phase increases. In this

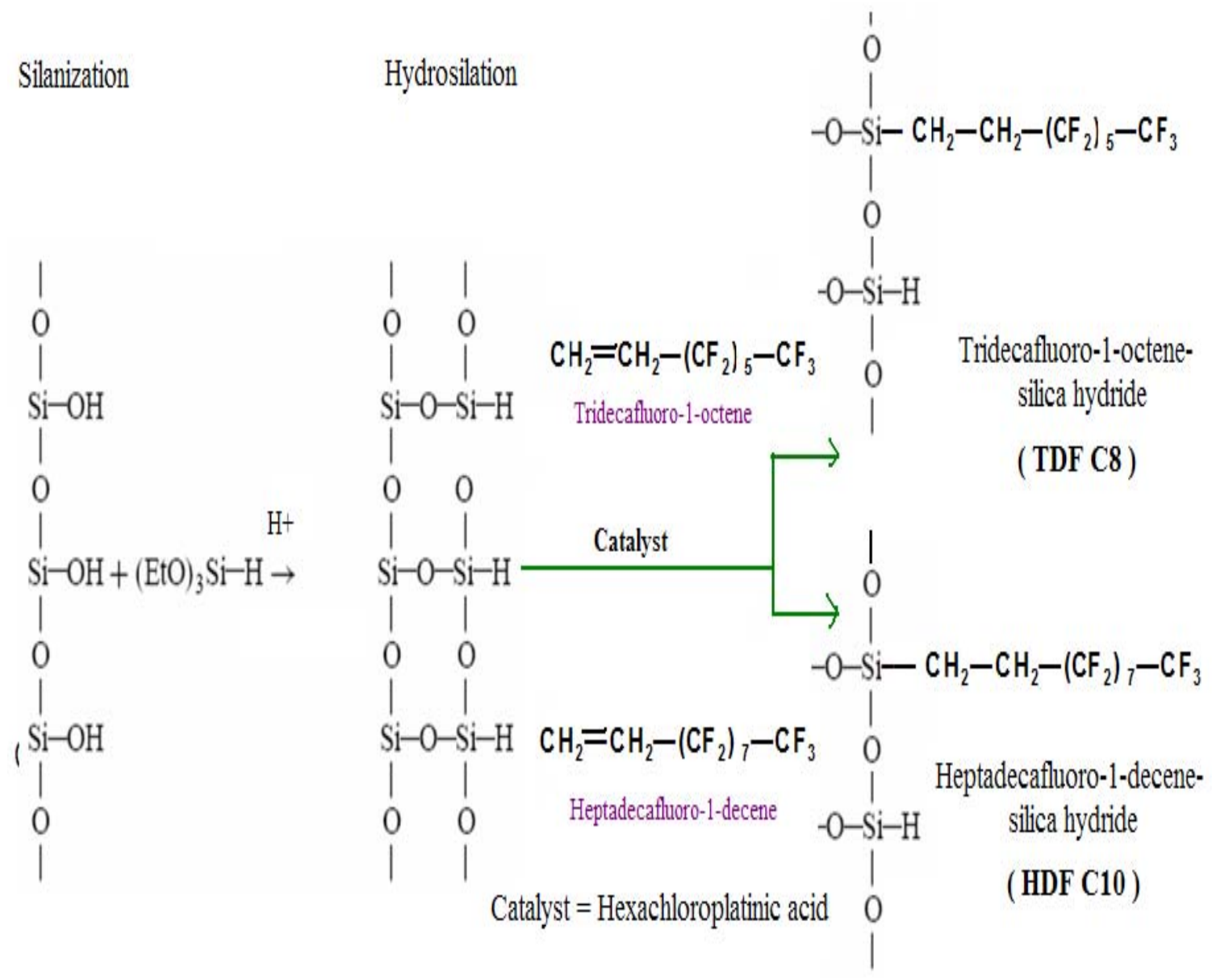

Figure 6. Synthesis of perfluoro hydride-based stationary phases using silanization and hydrosilation procedures. 
profile, the left side resembles reversed phase behavior and the right side resembles normal phase behavior [16]. This phenomenon of the fluorinated stationary phases resembles the silica hydride based aqueous normal phase mechanism used for polar basic retention. The notable difference between the fluorinated silica hydride phases in this study and those reported earlier in the literature would be the backbone of the silica, where to date the fluorinated phases studied are bonded to the silica with a significant number of silanols remaining on the surface. The hydride based columns have approximately $95 \%$ of the silanols replaced by the silica hydride compared to bare silica [13]. Therefore, this research has been designed to study the similar polar retentive mechanisms of fluorinated stationary phases on the hydrophobic silica hydride backbone using ANP chromatography with the hope of a synergistic outcome.

Figure 6 illustrates the silanization and hydrosilation procedures forming tridecafluoro-1-octene silica hydride and heptadecafluoro-1-decene silica hydride from the respective olefins. These phases are abbreviated as TDF C8 and HDF C10 respectively in the following sections.

\section{H. Characterization Techniques}

The silica hydride and hydride-based bonded stationary phases can be characterized using elemental analysis, Infrared (IR) and Nuclear Magnetic Resonance (NMR) spectroscopic techniques. The following sections describe the methods used in this investigation.

\section{Diffuse Reflectance Infrared Fourier Transform Spectroscopy (DRIFT)}

Diffuse reflectance infrared Fourier transform spectroscopy (DRIFT) is the semi- 
quantitative spectral analysis method that confirms the presence of the functional groups of the hydride and hydride based bonded phases. Nevertheless, the completion of the silanization and hydrosilation as well as the presence of adsorbed/bonded raw materials or final products can be explained qualitatively using DRIFT.

For solid, IR spectroscopy is a useful technique to characterize higher surface area materials. However, as the surface area decreases, it requires the Fourier transform method to improve the absorption sensitivity by signal averaging. Diffuse reflectance is another way of increasing the sensitivity of solid analyte absorption bands. In this study, DRIFT analysis is performed by irradiating the analytes mixed with $\mathrm{KBr}$ that is put in a small cup. The reflected light from the surface of the analytes is collected by a mirror and focused on to the detectors like mercury cadmium telluride (MCT) or deuterated triglycine sulfate (DTGS). The enhanced signal contains the fingerprint information of the analyte-bonded or adsorbed onto the surface [10].

\section{Carbon Elemental Analysis}

Elemental analysis is the combustion method that is used to determine the chemical composition of organic compounds. The silica-based bonded surfaces can be characterized quantitatively using the carbon elemental analysis. The fact that the silica moiety does not contain any traces of carbon means that if there is any carbon, it must be from the bonded moiety. Besides carbon, the other elements if present in the bonded organic moieties like nitrogen or fluorine can also be estimated using elemental analysis. In carbon elemental analysis, the amount of bonded moiety on the silica surface is 
calculated as the surface coverage $(\alpha)$ in the form of $\mu \mathrm{mol} / \mathrm{m}^{2}$ using the following Berendsen and de Galan equation:

$$
\alpha\left(\mu \mathrm{mol} / \mathrm{m}^{2}\right)=10^{6} p \mathrm{c} /\left(10^{2} \mathrm{M}_{\mathrm{C}} \mathrm{n}_{\mathrm{C}}-p_{\mathrm{C}} \mathrm{M}_{\mathrm{R}}\right) \mathrm{S}_{\mathrm{BET}}
$$

$p_{\mathrm{C}}-$ carbon $\%$ of the bonded material

$\mathrm{n}_{\mathrm{C}}$ - number of carbon atoms in the bonded organic group

$\mathrm{M}_{\mathrm{C}}$ - atomic weight of the carbon

$M_{R}$ - molecular weight of the organic compound

$\mathrm{S}_{\mathrm{BET}}$ - specific surface area of the silica material

It is a common trend to get lower surface coverage values as the size of the bonded organic moiety increases $[12,20]$.

\section{Nuclear Magnetic Resonance Spectroscopy (NMR)}

Nuclear Magnetic Resonance Spectroscopy (NMR) is a powerful spectroscopic tool that is mainly used for determining the structure of the organic compounds and for characterizing newly synthesized compounds. For the study of surfaces, solid state NMR is an accepted and frequently used method.

In general, solid state NMR gives a broad band for each NMR active nucleus of the same type due to its fixed magnetic orientations in the solid state in comparison with that in the liquid state. This broad range continuous chemical shift effect is known as chemical shift anisotropy (CSA) which can be overcome by utilization of magic angle spinning (MAS). Spinning the solid material very rapidly $(1-5 \mathrm{kHz})$ in the orientation of the diagonal of the cube (i.e., $55^{\circ}$, the magic angle), averages out the chemical shift variations; and the solid simulates as if it is a liquid, resulting in narrower bands. To 
study ${ }^{29} \mathrm{Si}$ and ${ }^{13} \mathrm{C}$ NMR active nuclei, the cross-polarization $(\mathrm{CP})$ criteria is required as well, which can transfer the NMR sensitivity from highly sensitive proton nuclei to ${ }^{29} \mathrm{Si}$ or ${ }^{13} \mathrm{C}$ nuclei that have lower sensitivity $[10,12]$. This research includes solid state CPMAS NMR study of carbon-13 as well as silicon-29 nuclei, the liquid state NMR of fluorine-19, and the slurry state NMR of H-1 to understand the changes occurring on the silica surface during the silanization and hydrosilation procedures.

\section{Types of Samples Used in the Evaluation of Columns}

In this research, small polar molecules are targeted as test probes because they exist in biomolecules and pharmaceutical compounds. Studying smaller molecules provides a better understanding of retention mechanisms which eventually may be applied to biological macromolecules [2]. Therefore, to study the ANP and RP dual retention behavior of the hydride based fluorinated columns, small polar molecules were selected that are described as follows.

First, amino acids were studied because they make up peptides and proteins. Amino acids exist as zwitterions at normal physiological $\mathrm{pH}(6-8)$ due to their one or more amino and carboxyl groups at the terminal positions. Due to this multiple polarity, they are hard to separate by reversed phase columns; pre-column or post-column derivatization are sometimes used to separate them on reversed phase columns. However, ANP's ability of separating polar and nonpolar molecules makes the amino acids an ideal choice of solute to study. On the basis of their net charge at neutral $\mathrm{pH}$, they can be categorized as acidic, basic or neutral amino acids [2]. In this study, basic arginine and 
histidine, acidic aspartic acid and glutamic acid, and neutral phenylalanine and tyrosine were analyzed using ELSD and MS detectors.

The other classes of compounds in this study include nucleobases, nucleosides and a nucleotide that are the main part of DNA and RNA. They have been reported to be separated by ion-exchange or reversed phase chromatography. However, the reversed phase mode gives very low retention and an ion-exchange mode utilizes aggressive buffers and salts in the mobile phase that are not suitable for using an MS detector [21]. Hence, they are very well suited solutes to try using ANP chromatography. These solutes were studied on ELSD and UV detectors.

The very polar and hard to separate creatine and creatinine were analyzed using ELSD. Creatinine is the breakdown product of creatine, mainly seen in the energy producing cycle of a human body. Creatine is known as an ergogenic acid -a nutritional supplement popular among athletes. They are the main components of human urine. Their levels in urine are commonly checked to evaluate human kidney function. Hence, they are good target solutes for ANP study [22, 23].

Another class of metabolites investigated is the small polar organic acids like maleic acid, fumaric acid, succinic acid and citric acid. They are highly polar and an essential part of biological metabolites. Since they are also challenging to separate, they are selected as a probe for ANP analysis using an MS system.

The final class of compounds tested is the carbohydrates. Monosaccharides (e.g., glucose, fructose, and ribose) and disaccharides (e.g., sucrose, lactose, maltose, trehalose, and turanose) were tested for ANP retention, and nonpolar disaccharide cellobiose 
octaacetate was tested for RP retention. Sugars are a difficult class of analytes to retain and to separate due to their high polarity and chemical structure similarities [2]. They are also a troublesome class of compounds to detect, due to lack of chromophores, and therefore they were detected with an MS system.

\section{J. Research Goals}

One objective of this research is to synthesize silica hydride based fluorinated stationary phases. Silanization synthesis was used to convert the silanols of silica to silica hydride. Subsequent hydrosilation of the silica hydride using perfluorinated C8 and C10 olefins introduced the fluorinated moieties on to it. Not all the hydrides were replaced by the fluorinated moieties due to steric reasons, and therefore these phases carry an underlying hydride monolayer contributing to the retention mechanisms.

Another goal includes the characterization of the bonded phases using spectral and elemental analyses. DRIFT spectral analysis validated the presence of hydride on the Astrosil silica surface after silanization. When the fluorinated moieties were attached, the intensity of the silica hydride band decreased and new bands appeared for $\mathrm{C}-\mathrm{H}$ stretching confirming the attachment of the fluorinated phases. Further, carbon elemental analysis estimated the amount of TDF C8 and HDF C10 attached to the silica hydride material. Finally, the attachment of hydride and fluorinated moieties on the silica matrix were confirmed qualitatively using proton-1, carbon-13, silicon-29, and fluorine-19 NMR studies. For the carbon and silicon spectra, ${ }^{29} \mathrm{Si}$ and ${ }^{13} \mathrm{C} \mathrm{CP}-\mathrm{MAS}$ solid state NMR were performed by our collaborator in Germany (Professor Klaus Albert, University of Tubingen). 
A third objective is the evaluation of the silica hydride based fluorinated bonded phases using ANP and RP dual modes using HPLC for polar basic analytes. The small polar molecules like amino acids, nucleobases, nucleotide, nucleosides, small polar organic acids, and carbohydrates were targeted to investigate their retention on these columns. Considering the detection requirements of these compounds and the availability of systems in the laboratory; ELSD, UV and MS detectors were used. Generally, ANP retention is demonstrated as the retention map of an individual solute by plotting its retention time versus concentration of mobile phase composition. Likewise, the retention of analytes on both columns (packed with TDF C8 and HDF C10) were studied and compared to predict whether the fluorine moiety or the hydride surface contributes to the retention. A reproducibility study was also performed to evaluate the column performance. 


\section{EXPERIMENTAL}

\section{A. Materials}

The chemicals used in the preparation of stationary phases and mobile phases utilized in this research are tabulated in this section. The small polar metabolites used in the evaluation of columns are also listed in the following tables.

Table 1. Chemicals used in the synthesis of stationary phases

\begin{tabular}{|c|c|}
\hline Chemical Name & Manufacturing Company \\
\hline Tridecafluoro-1-octene \\
(CAS\# 25291-17-2) & Aldrich Chemical Co. \\
St. Louis, MO \\
\hline Heptadecafluoro-1-decene & Aldrich Chemical Co. \\
(CAS\# 21652-58-4) & St. Louis, MO \\
\hline Astrosil Silica & Stellar Phases Inc. \\
& Langhorne, PA \\
\hline Hexachloroplatinic acid & Aldrich Chemical Co. \\
& St. Louis, MO \\
\hline Dioxane & Fisher Chemicals \\
& Fair Lawn, NJ \\
\hline Triethoxysilane & Aldrich Chemical Co. \\
& Milwaukee, WI \\
\hline Toluene & Fisher Chemicals \\
& Fair Lawn, NJ \\
\hline Diethyl ether & Fisher Chemicals \\
& Fair Lawn, NJ \\
\hline
\end{tabular}


Table 2. Chemicals used in the preparation of the mobile phases

\begin{tabular}{|c|c|}
\hline Chemical Name & Manufacturing Company \\
\hline Acetonitrile & Fisher Chemicals \\
& Fair Lawn, NJ \\
\hline Formic acid & $\begin{array}{c}\text { Spectrum Mfg. Corp. } \\
\text { Gardena, CA }\end{array}$ \\
\hline Ammonium formate & $\begin{array}{c}\text { Matheson Coleman \& Bell, } \\
\text { Rutherford, NJ }\end{array}$ \\
\hline
\end{tabular}

Table 3. Samples analyzed for the aqueous normal phase HPLC retention studies

3.1. Nucleobases, nucleosides, nucleotide, and ergogenic acids

\begin{tabular}{|c|c|}
\hline Chemical Name & Manufacturing Company \\
\hline Cytosine & Nutritional Biochemical Corp. \\
Cleveland, OH
\end{tabular}


3.2. Amino acids

\begin{tabular}{|c|c|}
\hline Chemical Name & Manufacturing Company \\
\hline Arginine & Matheson Coleman \& Bell \\
& Cincinnati, Ohio \\
\hline Histidine & $\begin{array}{c}\text { Sigma Chemical Co. } \\
\text { St. Louis, MO }\end{array}$ \\
\hline Aspartic acid & Mallinckrodt Inc. \\
& Paris, KY \\
\hline Glutamic acid & Sigma Chemical Co. \\
& St. Louis, MO \\
\hline Phenylalanine & J.T. Baker Chemical Co. \\
& Phillipsburg, NJ \\
\hline Tyrosine & Pierce chemical Co. \\
& Rockford, IL \\
\hline
\end{tabular}

\subsection{Organic acids}

\begin{tabular}{|c|c|}
\hline Chemical & Manufacturing Company \\
\hline Maleic acid & Sigma Chemical Co. \\
& St. Louis, MO \\
\hline Fumaric acid & Matheson Coleman \& Bell \\
& Cincinnati, Ohio \\
\hline Succinic acid & Mallinckrodt chemical works \\
& St. Louis, MO \\
\hline Citric acid & Mallinckrodt chemical works \\
& St. Louis, MO \\
\hline
\end{tabular}




\subsection{Carbohydrates}

\begin{tabular}{|c|c|}
\hline Chemical Name & Manufacturing Company \\
\hline Glucose & $\begin{array}{c}\text { Matheson Coleman \& Bell } \\
\text { Cincinnati, OH }\end{array}$ \\
\hline Fructose & $\begin{array}{c}\text { ICN Pharmaceuticals Inc. } \\
\text { Cleveland, OH }\end{array}$ \\
\hline Sucrose & J.T. Baker Chemical Co. \\
& $\begin{array}{c}\text { Phillipsburg, NJ } \\
\text { Lactose }\end{array}$ \\
\hline Maltose & Pfanstiehl Laboratories Inc. \\
& Waukegan, IL \\
\hline d-Ribose & J.T. Baker Chemical Co. \\
& Phillipsburg, NJ \\
\hline Turanose & Nutritional Biochemical Corp. \\
& Cleveland, OH \\
\hline d-Trehalose dehydrate & ICN Pharmaceuticals Inc. \\
& Cleveland, OH \\
\hline Cellobiose octaacetate & Matheson Coleman \& Bell \\
& Cincinnati, OH \\
\hline & ICN Pharmaceuticals Inc. \\
& Cleveland, OH \\
\hline
\end{tabular}




\section{B. Synthetic Procedures}

\section{Silanization}

The silanization synthesis was carried out to prepare silica hydride from silica. First, the required glassware was washed and dried in an oven overnight before the synthesis. Around the same time, $15.00 \mathrm{~g}$ of Astrosil silica was dried in the vacuum oven at $120{ }^{\circ} \mathrm{C}$ overnight. On the day of the synthesis, a $1000 \mathrm{~mL}$ three necked round bottom (RB) flask was fitted with a condenser with a drying tube, an addition funnel with a stopper, and a thermometer. This apparatus was put into the heating mantle which had been placed on a stir plate. The magnetic stir bar was put inside the RB flask. Later on, the dried silica was transferred to this RB flask, followed by an addition of $600 \mathrm{~mL}$ of dioxane and 19.44 $\mathrm{mL}$ of $2.3 \mathrm{M} \mathrm{HCl}$ solution, and lastly this reaction mixture was heated to $70{ }^{\circ} \mathrm{C}$. Meanwhile, $90.09 \mathrm{~mL}$ of dioxane was added into the addition funnel equipped with a stopper followed by addition of $20.91 \mathrm{~mL}$ of triethoxysilane (TES) in the presence of argon. Once the temperature remained constant at $70{ }^{\circ} \mathrm{C}$, the TES-dioxane mixture was added drop by drop into RB flask with constant stirring. After all the TES mixture was added, the temperature was raised to $90{ }^{\circ} \mathrm{C}$ and the reaction mixture was refluxed for 90 minutes.

Afterwards, this reaction product was allowed to cool down and transferred carefully into a medium size filter crucible attached to a filtering flask. The product was filtered using vacuum. This filtered product was washed two times with dioxane $(50 \mathrm{~mL}$ each time), three times with toluene ( $50 \mathrm{~mL}$ each time), and three times with diethyl ether (50 $\mathrm{mL}$ each time) using the same vacuum. Once washing was done, the final silica hydride 
product was dried at room temperature overnight to evaporate the ether. Finally, it was transferred into a beaker for drying in a vacuum oven at $110^{\circ} \mathrm{C}$ overnight.

\section{Hydrosilation}

The hydrosilation procedure was used to bond fluorinated moieties onto the silica hydride surface. All the required glassware for the synthesis was dried in the oven overnight. Then $2.00 \mathrm{~g}$ of silica hydride synthesized as described above was weighed and put in the vacuum oven at $110{ }^{\circ} \mathrm{C}$ overnight. A $250 \mathrm{~mL}$ three necked round bottom flask was attached to a condenser with a drying tube, a thermometer, and a stopper. This apparatus was put in a heating mantle which was placed on the magnetic stirrer in exactly the same way as discussed in the silanization procedure above. The magnetic stir bar was put inside the RB flask. Afterwards, $2.27 \mathrm{~mL}$ of tridecafluoro-1-octene $(1 \mathrm{H}, 1 \mathrm{H}, 2 \mathrm{H}$ perfluoro-1-octene) was dissolved in $80 \mathrm{~mL}$ of toluene and transferred into the RB flask. Subsequently, $1 \mathrm{~mL}$ of $10 \mathrm{mM}$ hexachloroplatinic acid in isopropanol was added to the reaction mixture into the $\mathrm{RB}$ flask and heated to $60{ }^{\circ} \mathrm{C}$ for 1 hour with constant stirring. Later on, the vacuum dried $2.00 \mathrm{~g}$ of silica hydride was added to the reaction mixture very slowly and the temperature was raised to $100{ }^{0} \mathrm{C}$. This temperature was maintained at $100{ }^{0} \mathrm{C}$ for 100 hours along with constant stirring. Once the reaction mixture was cooled down, it was filtered through a $40 \mathrm{M}$ size filter crucible using vacuum suction. The filtered solid was washed successively with $50 \mathrm{~mL}$ of toluene, $50 \mathrm{~mL}$ of dichloromethane and $50 \mathrm{~mL}$ of diethyl ether. This washed material (tridecafluoro-1octene silica hydride bonded phase) was kept at room temperature overnight to dry and later it was dried further in a vacuum oven at $110{ }^{0} \mathrm{C}$ for 24 hours. 
Similarly, $2.66 \mathrm{~mL}$ heptadecafluoro-1-decene $(1 \mathrm{H}, 1 \mathrm{H}, 2 \mathrm{H}$-perfluoro-1-decene) was mixed with $80 \mathrm{~mL}$ toluene and transferred to a $250 \mathrm{~mL}$ RB flask with exactly the same equipment described above. First, $1 \mathrm{~mL}$ of $10 \mathrm{mM}$ hexachloroplatinic acid in isopropanol was added into the reaction mixture and heated to $60{ }^{\circ} \mathrm{C}$ for 1 hour. Afterwards, $2.00 \mathrm{~g}$ of vacuum dried silica hydride was added carefully to it and the temperature was raised to $100{ }^{\circ} \mathrm{C}$ as described above. This reaction mixture was refluxed at $100{ }^{\circ} \mathrm{C}$ for 100 hours with constant stirring and later filtered and washed in a similar fashion. This heptadecafluoro-1-decene silica hydride bonded phase was kept at room temperature overnight to dry followed by vacuum oven drying at $100{ }^{0} \mathrm{C}$ for overnight.

\section{Instrumental Procedures}

\section{Diffuse Reflectance Infrared Fourier Transform Spectroscopy (DRIFT)}

To obtain DRIFT spectra, silica, silica hydride, and silica hydride based bonded phases as well as the reference material, $\mathrm{KBr}$, were first dried overnight in a vacuum oven at $110{ }^{0} \mathrm{C}$ prior to the analysis. The reference material $\mathrm{KBr}(5 \mathrm{mg})$ was mixed with the sample $(95 \mathrm{mg})$ by finely grinding them with a mortar and pestle. This mixture was transferred into a sample cup ( $3 \mathrm{~mm}$ diameter and $2 \mathrm{~mm}$ depth) which is a part of the diffuse reflectance accessory. The upper surface was smoothed with a spatula. Afterwards, this sample cup was placed inside the ATI Mattson Infinity Series FTIR ${ }^{\mathrm{TM}}$ spectrometer that is equipped with a deuterated triglycine sulfate (DTGS) detector. Before starting the analysis, the sample compartment was purged with nitrogen for 15 minutes to remove carbon dioxide and water. All the spectra were recorded using the 
WinFIRST $^{\mathrm{TM}}$ application software in the range of $4000-450 \mathrm{~cm}^{-1}$ and averaging 112 scans for each spectrum. First, $\mathrm{KBr}$ was scanned as a background reference. Then the spectra of Astrosil silica, silica hydride, tridecafluoro-1-octene silica hydride (TDF C8) and heptadecafluoro-1-decene silica hydride (HDF C10) were obtained.

\section{Carbon Elemental Analysis}

Small amounts ( 100 mg) of both fluorinated bonded phases, TDF C8 and HDF C10, obtained from the hydrosilation step, were sent to Columbia Analytical Services Inc., (Tucson, AZ) for carbon elemental analysis. A combustion method in the presence of a $\mathrm{WO}_{3}$ catalyst was used to determine the $\%$ carbon in the bonded phases. These values of $\% \mathrm{C}$ were used to calculate the surface coverage of the bonded phases on the silica material.

\section{Nuclear Magnetic Resonance Spectroscopy (NMR)}

Solid state CP-MAS ${ }^{29} \mathrm{Si}$ NMR and ${ }^{13} \mathrm{C}$ NMR for the synthesized tridecafluoro-1octene silica hydride (TDF C8) and heptadecafluoro-1-decene silica hydride (HDF C10) were performed by our collaborator in Germany (Professor Klaus Albert, University of Tubingen). Slurry state ${ }^{1} \mathrm{H}$ NMR and ${ }^{19} \mathrm{~F}$ NMR spectra were recorded on a Varian INOVA $400 \mathrm{MHz}$ NMR spectrometer system (with Glide software) to characterize Astrosit $^{\mathrm{TM}}$ silica, silica hydride, TDF C 8 and HDF C10. For slurry state analysis, $\sim 40 \mathrm{mg}$ of sample was mixed with deuterated methanol $\left(\mathrm{CD}_{4} \mathrm{OD}\right)$ and transferred to a $5 \mathrm{~mm}$ o.d. glass tube (Wilmad Glass Co., Inc.). This mixture was sonicated for 5 minutes to make the slurry. The glass tube holding the NMR sample slurry was carefully fixed in the sample spinner and was placed in the spectrometer to spin overnight before acquiring the 
data. The solvent, deuterated methanol-D4 (D 99.8\%, catalog number $=$ DLM-24) was obtained from Cambridge Isotope Laboratories Inc. (CIL, Andover, MA). Solution state

${ }^{19} \mathrm{~F}$ NMR was performed to check the purity of the substrate olefins: tridecafluoro-1octene and heptadecafluoro-1-decene, using deuterated methanol-D4 (D 99.8\%, catalog number $=$ DLM-24, CIL- Cambridge Isotope Laboratories Inc., Andover, MA). For each measurement, the NMR instrument was locked using the deuterium solvent signal for these qualitative studies.

\section{High Performance Liquid Chromatography (HPLC)}

The HPLC instrument used in this research consisted of a Hewlett Packard Interface 35900- a multichannel interface, a Waters In-Line degasser, an LCD/Milton Roy CM4000 multiple solvent delivery system, a Rheodyne model 7126 manual injector (with $20 \mu \mathrm{L}$ injection loop), and a SofTA Corporation model 400 evaporative light scattering detector (ELSD with Thermo-Split technology). The ELSD detection system used a continuous stream of nitrogen gas (65 psi pressure) as a nebulization gas to form an aerosol cloud of the HPLC effluents. The ELSD had a $670 \mathrm{~nm}$ laser diode source and hermetically sealed photodiode detection equipment. All of the samples that utilized the ELSD detector were analyzed using a $0.5 \mathrm{~mL} / \mathrm{min}$ flow rate. The nebulization chamber temperature was optimized to $25{ }^{\circ} \mathrm{C}$ and the evaporative zone temperature was optimized to $55{ }^{\circ} \mathrm{C}$.

This same HPLC system attached to a Shimadzu SPD-6A UV spectrophotometer detector was utilized for UV absorbing samples. For the purpose of instrument control and data collection, Chemstation software was used. An HP Deskjet 932C printer was 
used to print the chromatograms. The deionized water used for mobile phases, was prepared on a Milli- $\mathrm{Q}^{\mathrm{TM}}$ purification system (Millipore Corp., Bedford, MA). For a majority of the samples analyzed by HPLC-UV, the mobile phase flow rate was 0.5 $\mathrm{mL} / \mathrm{min}$.

The second HPLC instrument used was an HP Series II 1090 mainframe liquid chromatograph interfaced with an LC/MS/MS PE SCIEX/API 300 (Applied Biosystem / MDS Sciex) mass spectrometer. This mass spectrometer was equipped with a TURBO IONSPRAY electrospray ionization (ESI) source. Although this PE SCIEX API Triple Quadrupole series instrument was capable of performing complex MS/MS analysis, only a single LC-MS i.e., [Q1 MS (Q1)] or [Q3 MS (Q3)] scan type was used to detect the analytes on the basis of mass to charge ratio. The constant flow of LN2 Dewar nitrogen gas (100 psi) was used for ESI nebulization and nitrogen gas with 70 psi pressure was used for the purpose of degassing the mobile phase. The Turbolon Ion Spray gas flow rate was adjusted to $5000 \mathrm{cc} / \mathrm{min}$. The gas source related parameters were set as follows using a manual tuning mode: the nebulizer gas (NEB) 13, curtain gas (CUR) 10, ion spray voltage (IS) 5000, temperature (TEM) 350 . The API system was calibrated using a poly(propylene)glycol tuning solution for both quadrupole analyzers Q1 and Q3 in both the positive and negative ionization modes. The Analyst 1.3 software was used to control the LC-MS unit and the Chemstation software was used to control HPLC unit. In LC-MS, the Analyst 1.3 software controlled the successive modes of operations of tuning the instrument, calibrating it, optimizing it for the analyte compound, running the samples, manipulating the data and saving them as projects. The flow rate used for this 
study was $0.4 \mathrm{~mL} / \mathrm{min}$ for all of the samples. The results of mass analysis were printed using an HP LaserJet $4050 \mathrm{~N}$ printer. The mobile phases used for all the samples, were vacuum filtered using a $0.45 \mu \mathrm{m}$ Nylon $47 \mathrm{~mm}$ membrane filter (catalog \# 2024, Alltech, Deerfield, IL). Both fluorinated stationary phases were packed into $100 \mathrm{~cm} \times 4.6 \mathrm{~mm}$ i.d. stainless steel columns (catalog: 9449 C) at SJSU using Alltech hardware (Alltech, Waukegan Road, Deerfield, IL). 


\section{RESULTS AND DISCUSSION}

\section{A. DRIFT Spectroscopic Characterization}

The success of silanization and hydrosilation synthetic procedures can be confirmed using DRIFT spectroscopy. Figure 7 shows the DRIFT spectrum of the silica hydride intermediate. The presence of the sharp peak at $2250 \mathrm{~cm}^{-1}$ frequency is due to the stretching vibrations of the newly formed Si-H functional group. This intense Si-H band confirms the success of the silanization procedure on the Astrosil silica . The broad peak from $3800 \mathrm{~cm}^{-1}$ to $3000 \mathrm{~cm}^{-1}$ is due to the combined contribution of adsorbed water and hydrogen bonded silanols. The sharper band around $3750 \mathrm{~cm}^{-1}$ is due to non hydrogen bonding silanols. The other noticeable bands of the spectrum are attributed to the various fundamental vibrations of the silica matrix.

The DRIFT spectrum of the tridecafluoro-1-octene silica hydride bonded phase is shown in Figure 8. This spectrum shows the presence of an aliphatic $\mathrm{C}-\mathrm{H}$ stretching band around $3000-2800 \mathrm{~cm}^{-1}$ that is evidence of the introduction of the fluorinated carbon moiety onto the silica hydride material. The intensity of the characteristic Si-H stretching band at $2250 \mathrm{~cm}^{-1}$ has diminished. This loss of Si-H signal provides a good indication of the attachment of the fluoro organic bonded moiety onto the silica hydride surface. Here, one can expect C-F stretching bands somewhere in the range of $1150-950 \mathrm{~cm}^{-1}$ for the fluorinated bonded moiety, but unfortunately, this frequency range is dominated by very intense siloxane bending absorption of the silica matrix. Therefore, it was not possible to observe any peak for C-F functional group. 
Similar results are observed for heptadecafluoro-1-decene silica hydride bonded phase as shown in the Figure 9. The reduced intensity of the Si-H peak at $2250 \mathrm{~cm}^{-1}$ and the appearance of $3000-2800 \mathrm{~cm}^{-1} \mathrm{C}-\mathrm{H}$ aliphatic peaks are indicative of the attachment of the heptadecafluoro-1-decene moiety to the silica hydride surface.

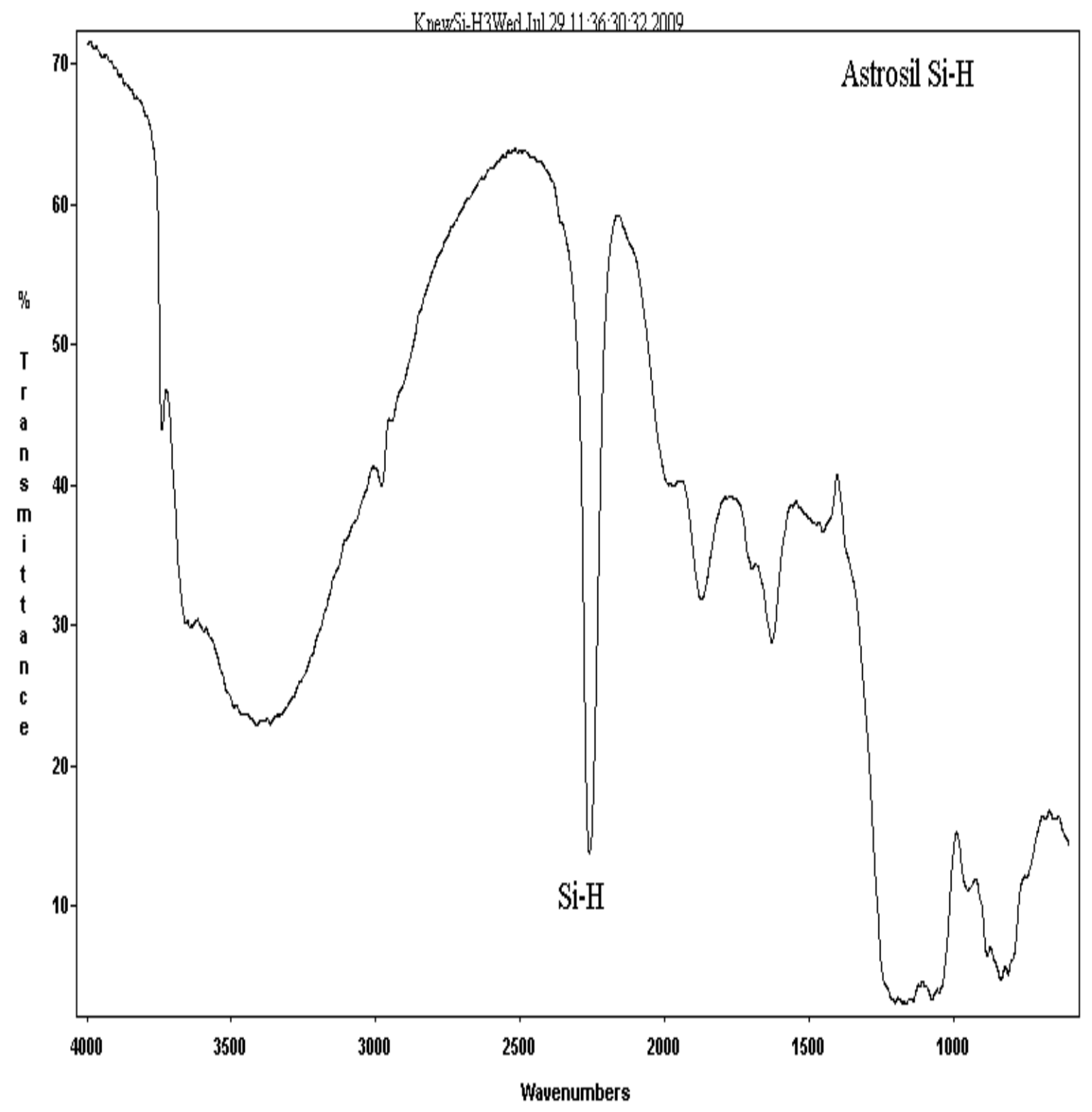

Figure 7. DRIFT spectrum of Astrosil silica hydride. 


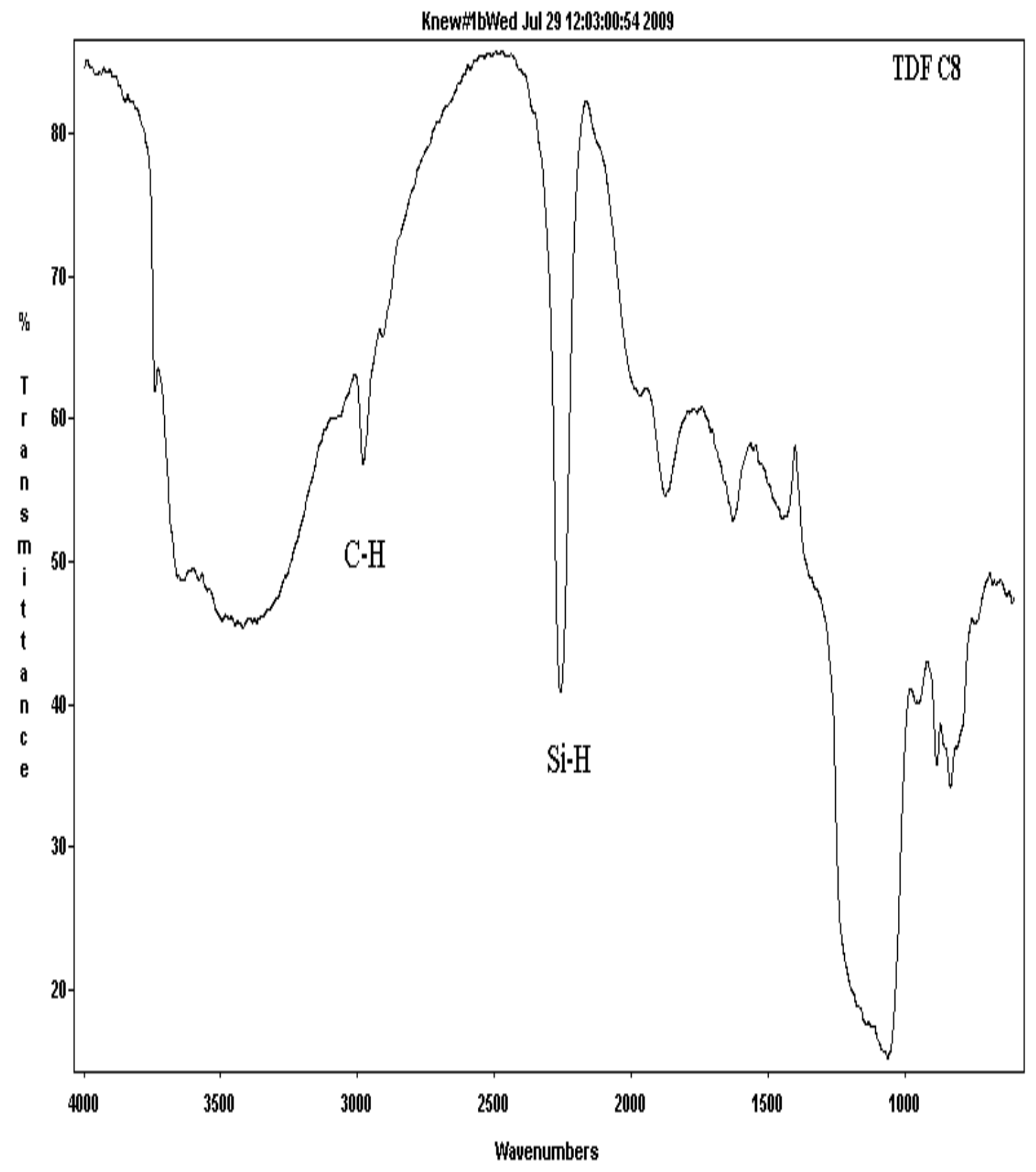

Figure 8. DRIFT spectrum of tridecafluoro-1-octene silica hydride. 


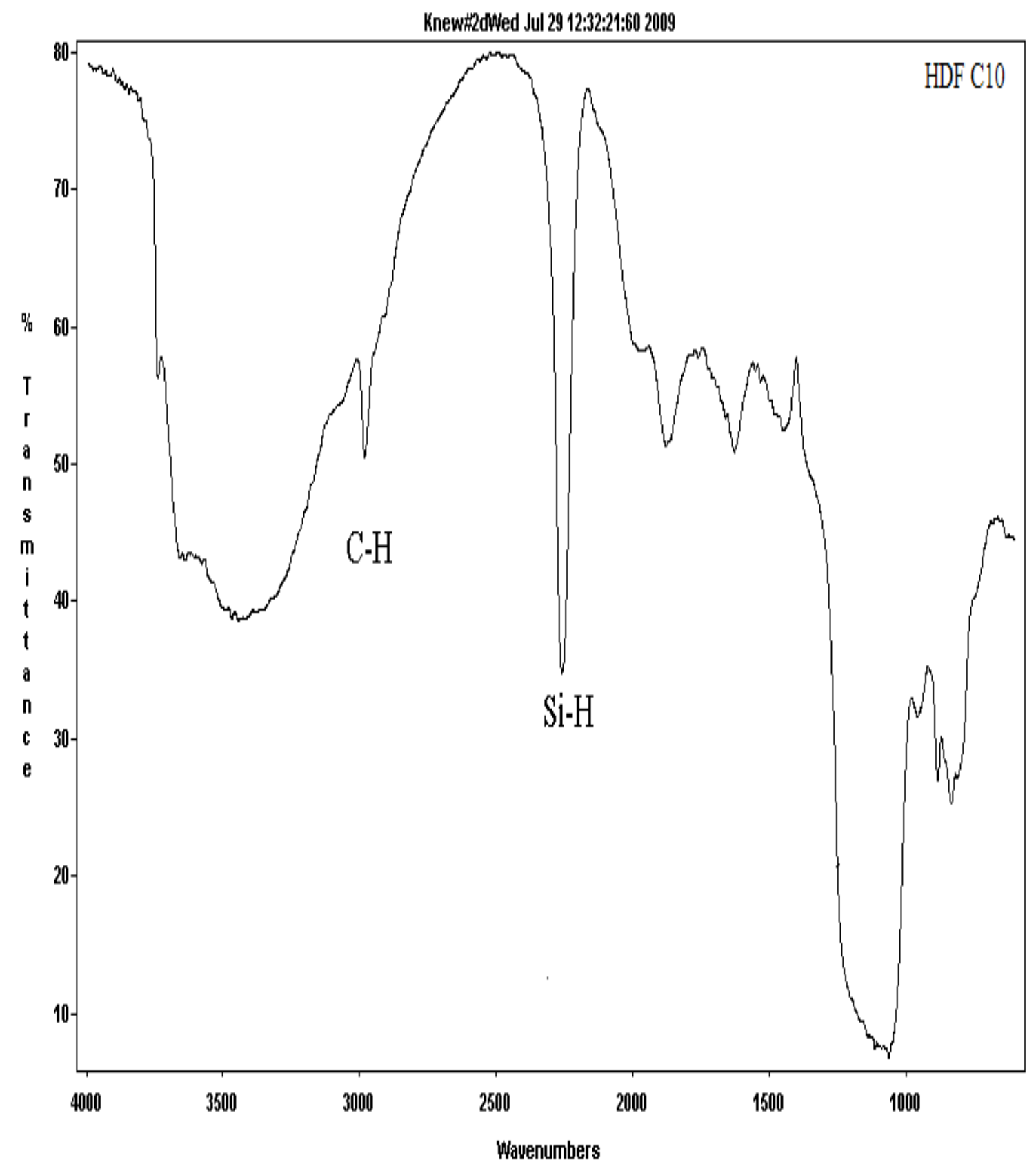

Figure 9. DRIFT spectrum of heptadecafluoro-1-decene silica hydride. 


\section{B. Carbon Elemental Analysis Predictions}

Carbon elemental analysis has been used to confirm the attachment of organic moieties as well as to estimate the amount of these bonded groups quantitatively. Table 4 shows the specific surface area value for Astrosil silica, the molecular formulas of the fluorinated olefins that have been attached to silica hydride, the percentage of carbon loading, and the surface coverage values in $\mu \mathrm{mol} / \mathrm{m}^{2}$ for tridecafluoro-1-octene silica hydride (TDF C8) and heptadecafluoro-1-decene silica hydride (HDF C10) fluorinated bonded phases. These surface coverage values were calculated using the previously described Berendsen and de Galan equation. The molecular weights of tridecafluoro-1octene and heptadecafluoro-1-decene used were $346.09 \mathrm{~g} / \mathrm{mol}$ and $446.11 \mathrm{~g} / \mathrm{mol}$ respectively.

Table 4. Surface coverage values of bonded organic moieties

\begin{tabular}{|c|c|c|c|c|c|}
\hline Silica & $\begin{array}{c}\mathrm{S}_{\mathrm{BET}} \\
\left(\mathrm{m}^{2} / \mathrm{g}\right)\end{array}$ & Bonding moiety & Molecular formula & $\mathbf{\% C}$ & $\begin{array}{c}\text { Surface } \\
\text { coverage } \\
\mu \mathrm{mol} / \mathrm{m}^{2}\end{array}$ \\
\hline Astrosil & 350 & $\begin{array}{c}\text { Tridecafluoro- } \\
\text { 1-octene }\end{array}$ & CF3-(CF2)5-CH=CH2 & 1.9 & 0.606 \\
\hline Astrosil & 350 & $\begin{array}{c}\text { Heptadecafluoro- } \\
\text { 1-decene }\end{array}$ & $\mathrm{CF} 3-(\mathrm{CF} 2) 7-\mathrm{CH}=\mathrm{CH} 2$ & 1.9 & 0.486 \\
\hline
\end{tabular}

Under the applied reaction conditions, the surface coverage values calculated for TDF C8 and HDF C10 bonded moieties are $0.606 \mu \mathrm{mol} / \mathrm{m}^{2}$ and $0.486 \mu \mathrm{mol} / \mathrm{m}^{2}$ respectively. The higher surface coverage value of TDF C8 than that of HDF C10 
supports previous results indicating that smaller olefins have higher bonding density than larger molecules. Because tridecafluoro-1-octene is smaller in size when compared with the heptadecafluoro-1-decene, it has relatively lower steric hindrance mainly due to the fluorine atoms resulting in higher amount of bonding on the silica hydride surface. Consequently, due to the fact that TDF C8 has a higher amount of organic fluoro moiety attached, it will have a lower amount of hydride contributing for possible interaction/ retention compared to HDF $\mathrm{C} 10$ bonded phase. Therefore, it is likely that the underlying silica hydride plays a greater role in the retention mechanism for the HDF C10 bonded phase than the TDF C8 bonded phase.

\section{Nuclear Magnetic Resonance Spectroscopic Confirmations 1. $\quad{ }^{29} \mathrm{Si} \mathrm{NMR}$}

The solid state NMR spectra of chemically modified silica surfaces have provided very useful information about the linkages formed between the modifying group and the silicon oxide surface. Figure 10 represents the silicon-29 CP-MAS NMR spectrum of the tridecafluoro-1-octene silica hydride bonded phase material. The readily observable peaks represent the silica matrix and the silica hydride layer. As shown in the spectrum, the highest field peak Q4 at -110 ppm is due to silicon with four siloxane linkages (i.e., the silica backbone). The Q3 peak at -101 ppm can be attributed to the isolated silanol moieties. The very intense peak around $-85 \mathrm{ppm}$ is due to the silicon atom attached to the hydride and three inner siloxane backbone linkages. This apparently intense peak of Si-H supports the formation of the silica hydride intermediate after the silanization procedure. Hence, it also confirms the DRIFT result of silica hydride formation. The peak at -75 
ppm is due to silicon atom attached to the hydride, a silanol and the siloxane linkages.

However, the most interesting peak at $-16 \mathrm{ppm}$ indicates the presence of fluorinated carbon organic moiety as Si-C linkage.

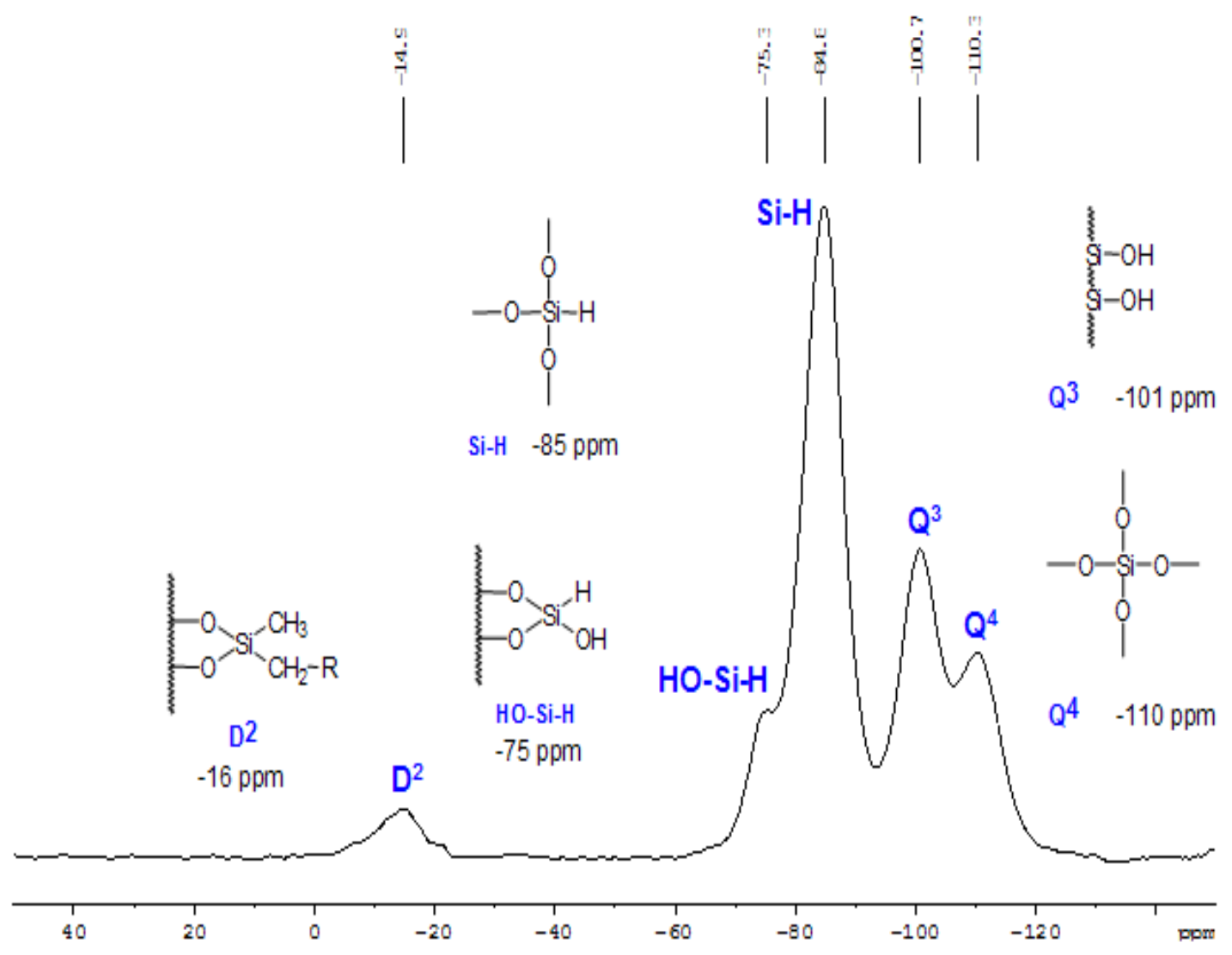

Figure 10. Silicon-29 CP-MAS NMR spectrum of TDF C8. 
Similar silicon-29 CP-MAS NMR chemical shifts have been observed for heptadecafluoro-1-decene silica hydride bonded phase material as shown in Figure 11. These results also collectively confirm the linkages of the siloxane backbone, the silanol, the hydride and the fluorinated carbon moiety attached to the silicon atoms of fluorinated bonded phases.

Heptadecafluoro-1-decene on Si-H

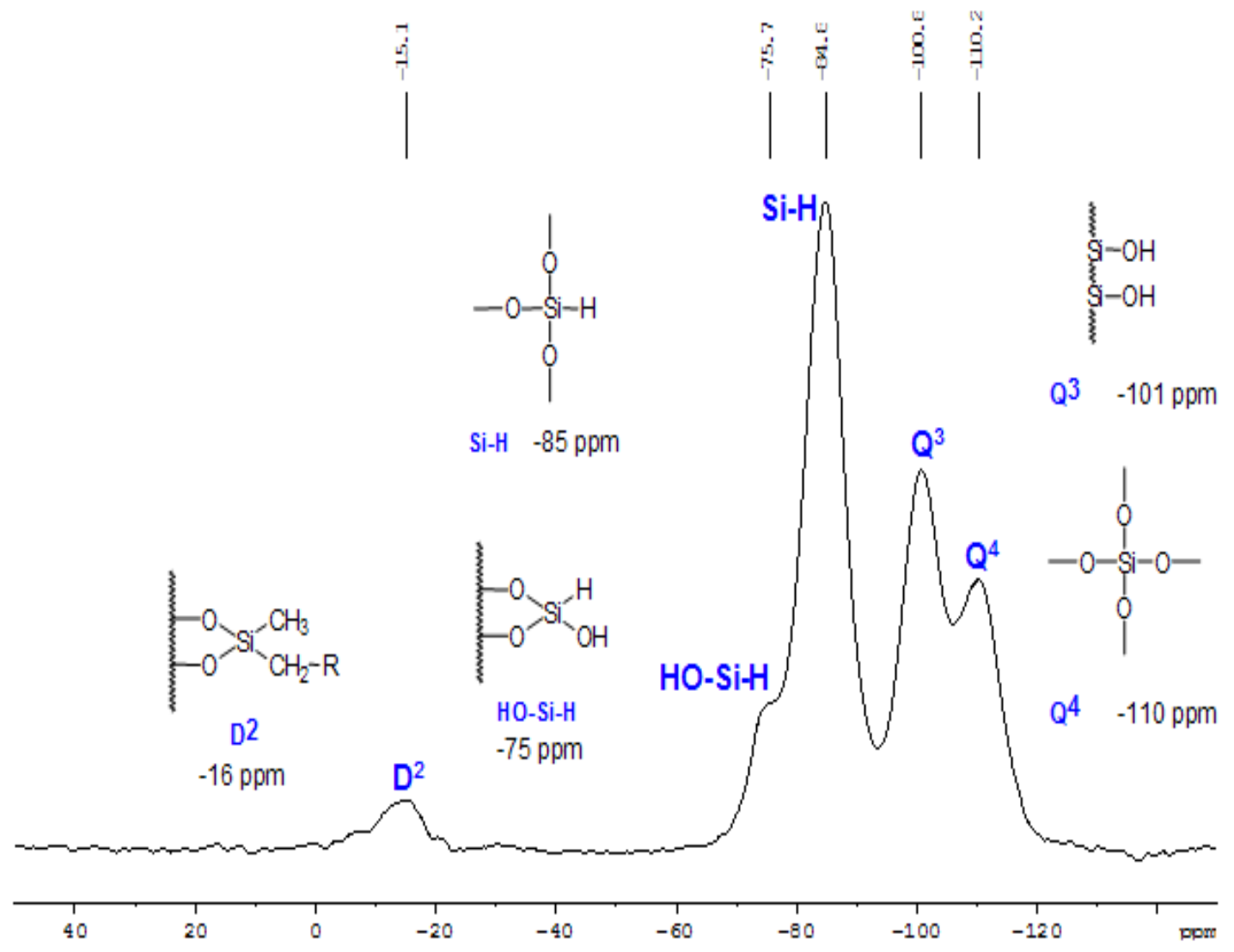

Figure 11. Silicon-29 CP-MAS NMR spectrum of HDF C10. 


\section{2. $\quad{ }^{13} \mathrm{C}$ NMR}

The carbon-13 CP-MAS NMR spectra of tridecafluoro-1-octene silica hydride and heptadecafluoro-1-decene silica hydride as shown in Figure 12 and Figure 13 respectively represent the resonances of individual carbon atoms of the hydrogenated part of the fluorinated organic bonded moiety. Although the peak widths are not quite as

\section{Tridecaluoro-1-octene on Si-H}
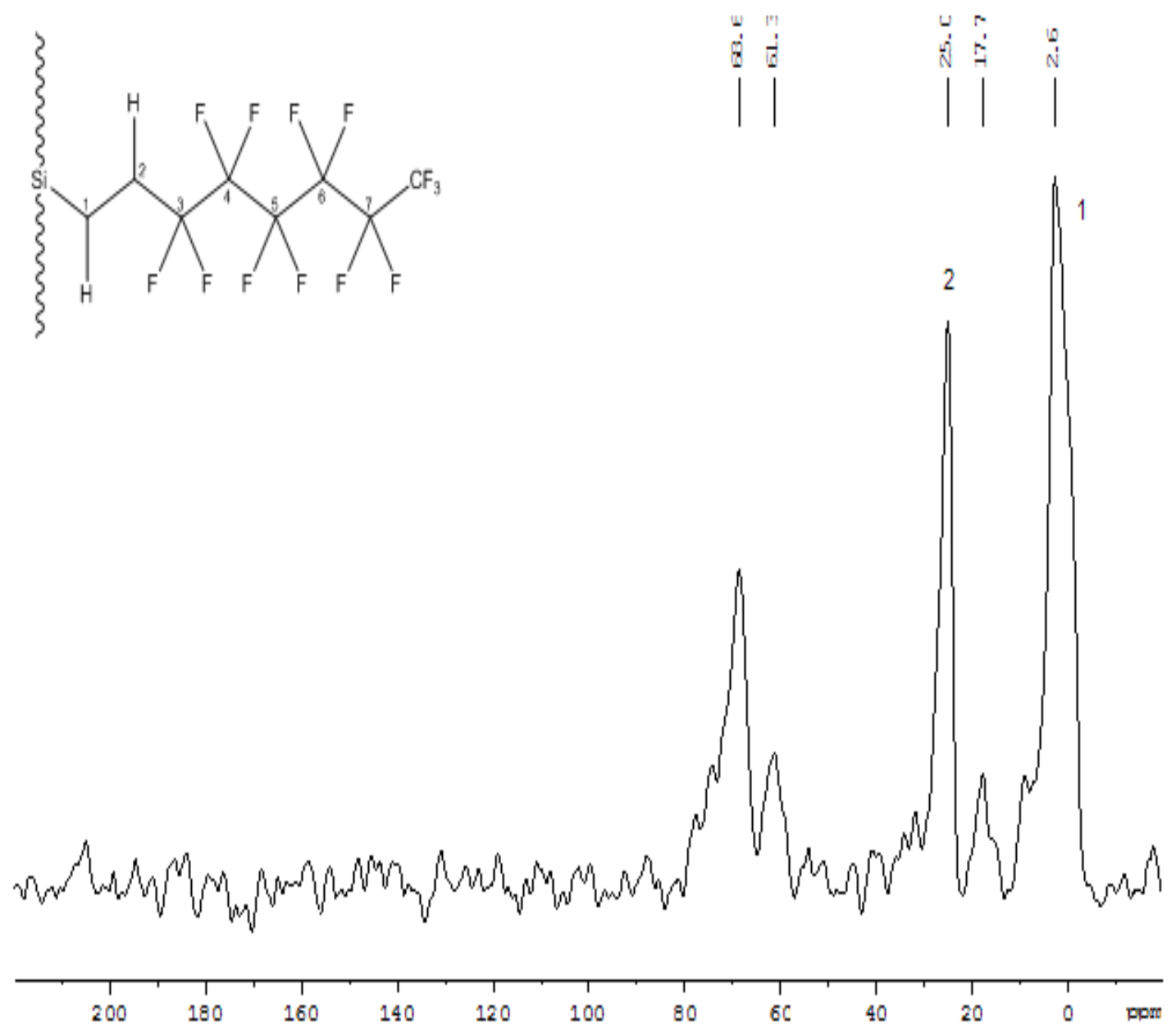

Figure 12. Carbon-13 CP-MAS NMR spectrum of TDF C8. 
Heptadecafluoro-1-decene on Si-H
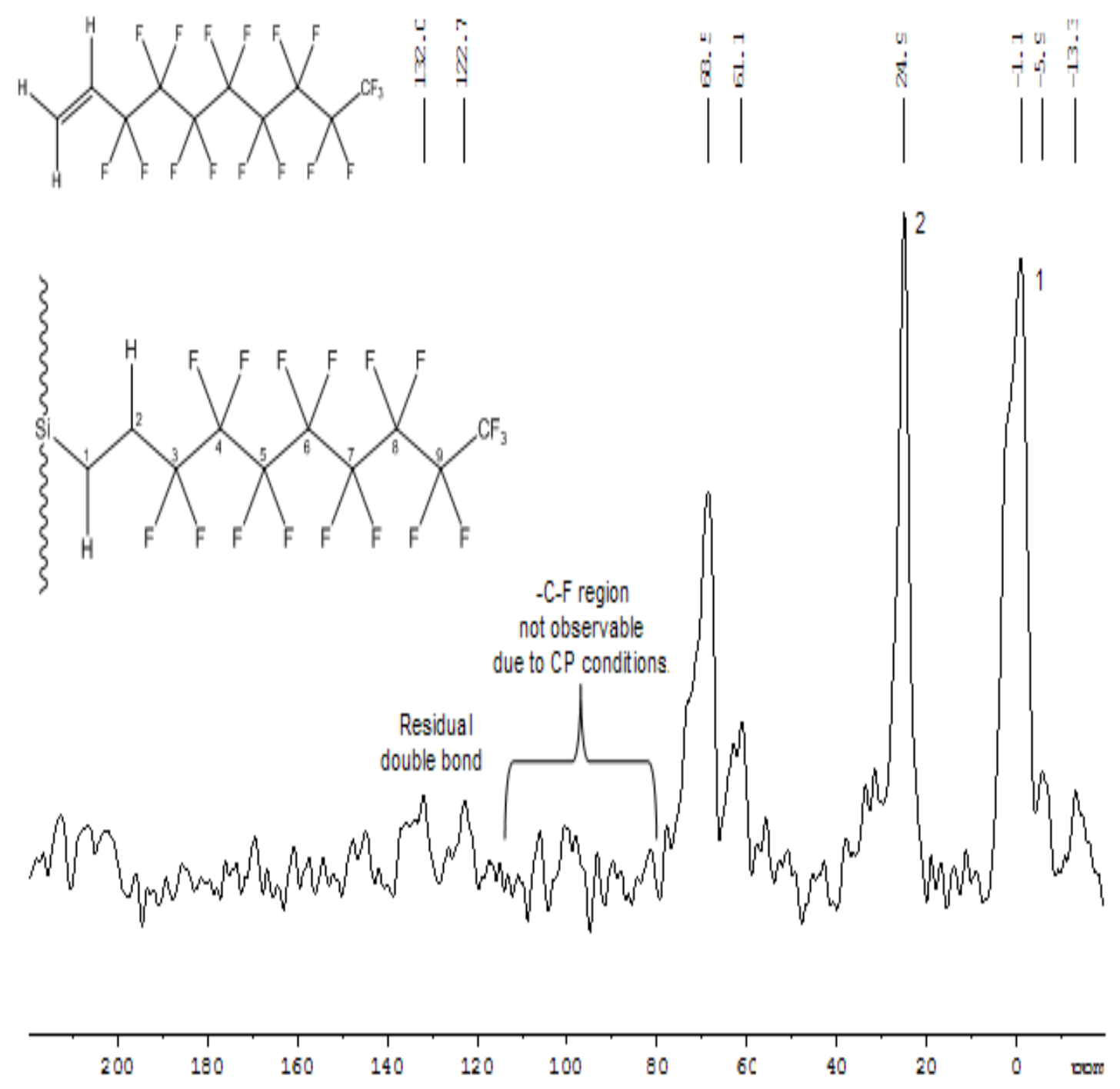

Figure 13. Carbon-13 CP-MAS NMR spectrum of HDF C10. 
narrow as those found in the solution spectra, the observed peaks are useful to identify the correct carbon environment confirming the hydrosilation bonding of the fluorinated carbon moiety on the silica hydride intermediate. Since the structures of TDF C8 and HDF C10 bonded moieties carry only two carbons with protons (they are the spacer carbons of the fluorinated bonded phases), only two obvious peaks are observed. The upfield peak 1 is due to the carbon atom $\mathrm{C} 1$ which is directly bonded to a silicon atom, and peak 2 is due to a carbon atom $\mathrm{C} 2$ positioned next to $\mathrm{C} 1$. The carbon-13 NMR can observe only proton bearing carbon atoms because the low abundance carbon nuclei have been irradiated with proton frequencies. Therefore Figure 12 of TDF C8 and Figure 13 of HDF $\mathrm{C} 10$ do not show any C-F chemical shifts. The additional comparatively small downfield peaks can be attributed to the residual ethoxy moiety of TES used in the silanization and the olefin substrate used in the hydrosilation. Thus, these carbon-13 NMR spectra also qualitatively confirm the bonding of the TDF C8 and the HDF C10 onto the silica hydride material.

\section{3. $\quad{ }^{1} \mathbf{H}$ NMR}

Due to the fact that proton-1 nucleus has higher sensitivity, a very dilute solution should be sufficient to reveal the structural information of organic compounds. The solid state proton-1 NMR on the other hand is more difficult to obtain as it requires application of different methods to remove the proton dipole-dipole interactions and the chemical shift anisotropy effects. However, this research has tried slurry state proton-1 NMR of silica, silica hydride, and the fluorinated organic bonded moiety for the first time. It 
should qualitatively confirm the success of the silanization and hydrosilation procedures on silica material.

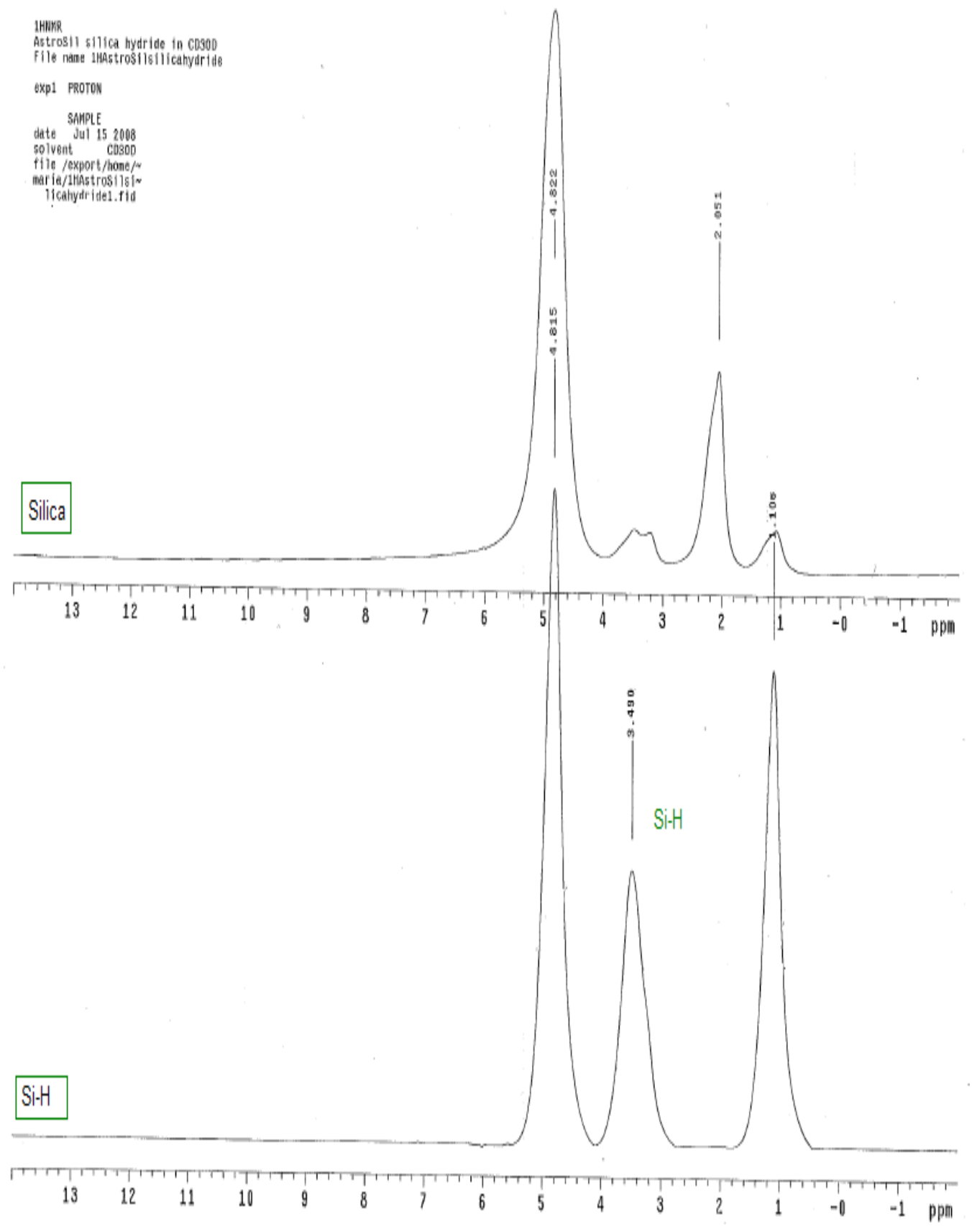

Figure 14. Proton-1 slurry state NMR spectra of Astrosil silica and silica hydride. 
Figure 14 represents the overlay of slurry state proton-1 NMR spectra of the Astrosil silica (on the top) and the silica hydride (on the bottom). The broad intense band at 4.82 ppm is due to the proton of water adsorbed onto the silica surface. The slurry was prepared in deuterated methanol that might have other solvent impurities like methanol, water or other proton carrying solvents. Hence the peak at $1.10 \mathrm{ppm}$ can be attributed to the proton of the impurity solvents. The middle peak at $2.05 \mathrm{ppm}$ can be assigned to the isolated silanol groups. The most important peak to note is a peak at $3.49 \mathrm{ppm}$ on the bottom part which is the peak of silica hydride group. As can be seen from the spectra, this peak is absent on the top spectrum of Astrosil silica, its appearance in the bottom spectrum confirms the attachment of hydride on the silica after the silanization step. The small peak at 3-4 ppm in the silica spectrum might be due to some solvent impurities. Figure 15 represents the slurry state proton NMR spectra of bonded material TDF C8 (on the top) and silica hydride (on the bottom). Both spectra show the bands for the silica hydride proton at around $3.30 \mathrm{ppm}$, adsorbed water proton at $4.80 \mathrm{ppm}$, and an impurity methanol proton at $1.20 \mathrm{ppm}$ as discussed earlier. The interesting new peak to monitor here is the peak at $2.13 \mathrm{ppm}$ which is absent in the hydride spectra. This new peak can be attributed to the protons of the spacer carbons of the tridecafluoro-1-octene olefin attached to silica hydride after the hydrosilation step. A similar fluoro bonded moiety proton band can be observed at $2.14 \mathrm{ppm}$ in the slurry state proton NMR of HDF C10 (Figure 16). One more point to observe is the intensity of the silica hydride band in Figure 15; it decreases in the top spectrum of TDF C8 when compared to the bottom silica hydride spectrum. This fact is indicative of the utilization of the silica hydride 


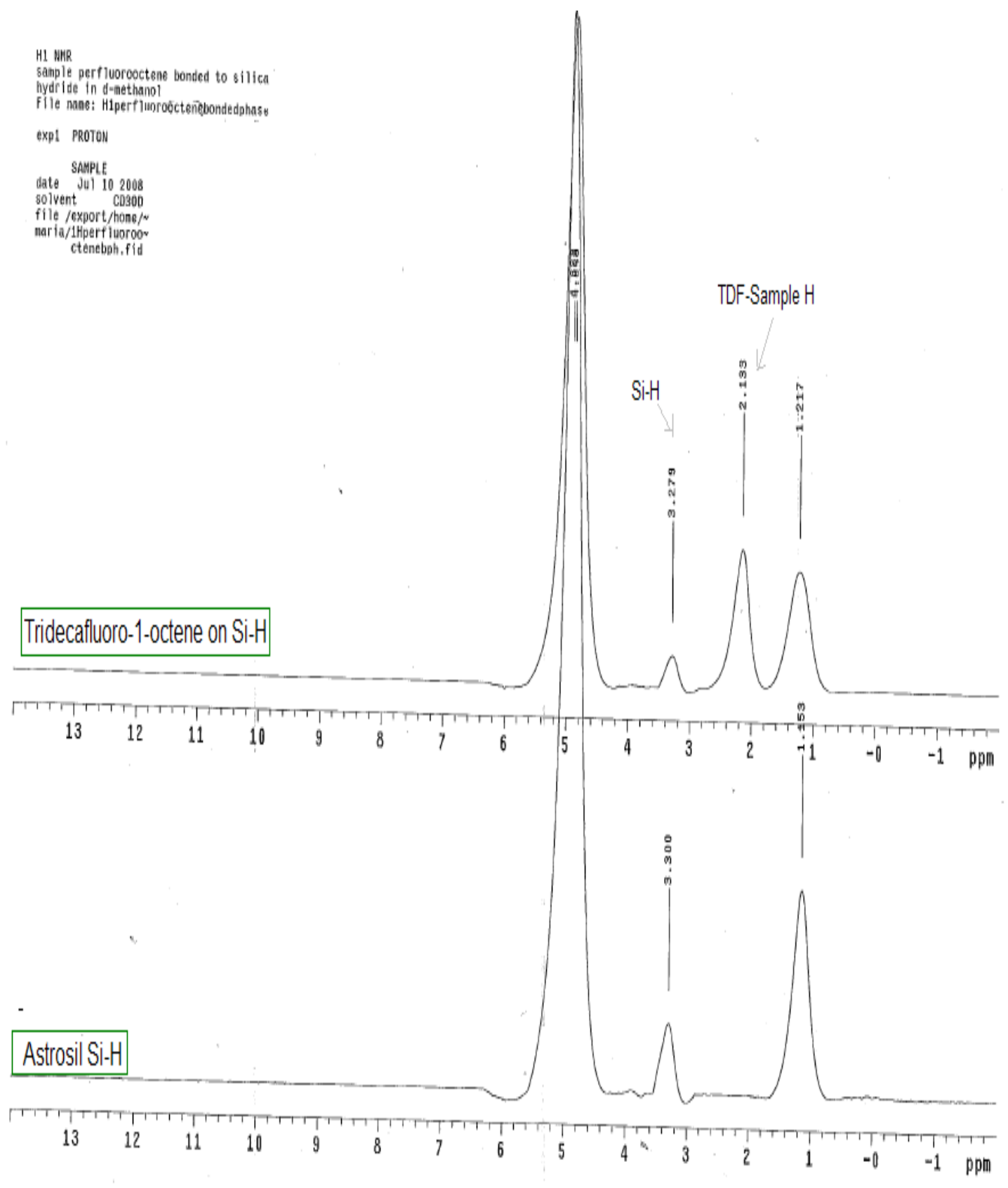

Figure 15. Proton-1 slurry state NMR spectra of TDF C8 and silica hydride. 


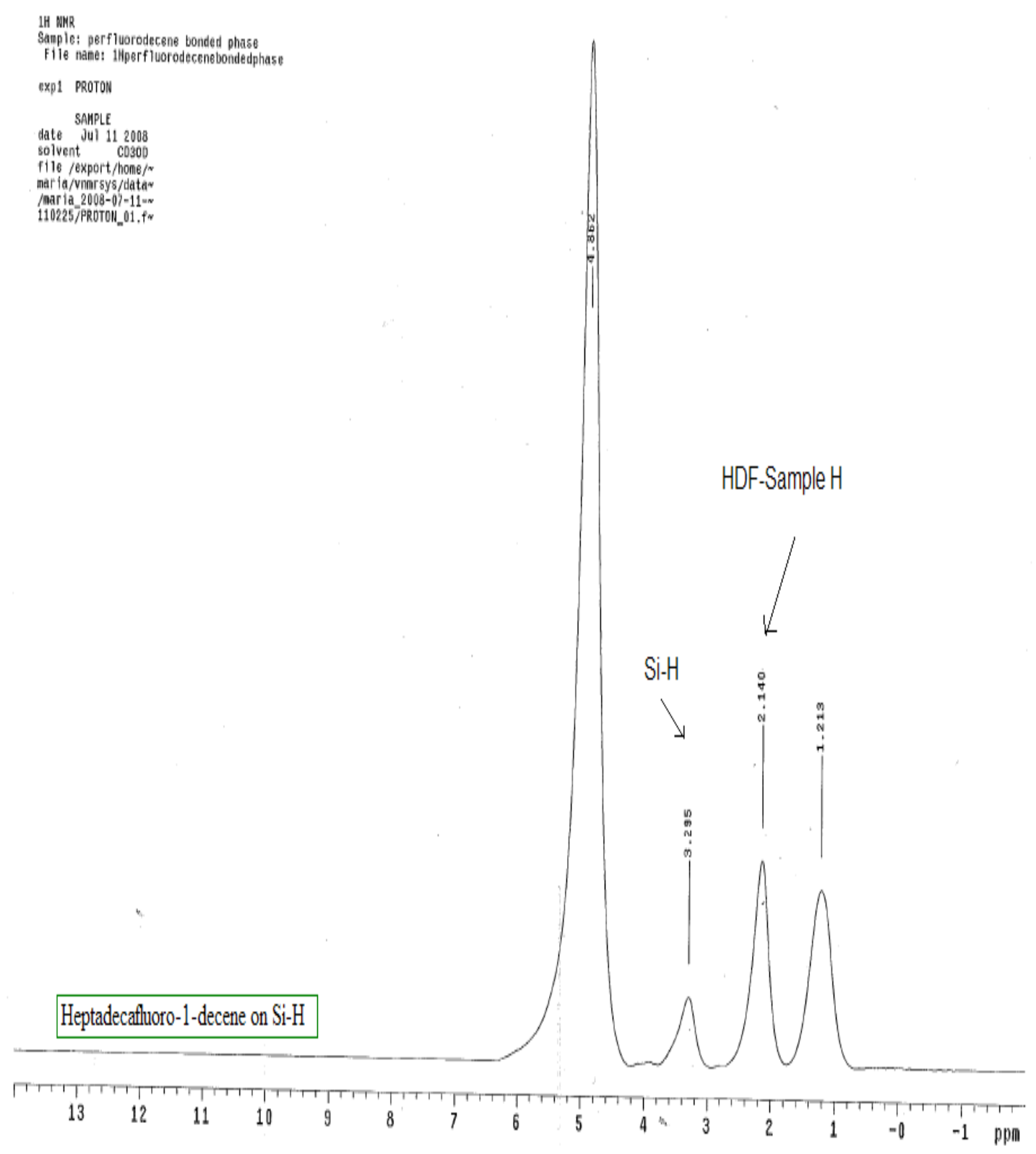

Figure 16. Proton-1 slurry state NMR spectrum of HDF C10.

layer for the formation of TDF C8 bonded phase. These slurry state proton NMR data collectively validates the fact that hydride as well as fluorinated organic functional groups have been bonded to the silica surface. 


\section{4. $\quad{ }^{19}$ F NMR}

For the hydrosilation step, tridecafluoro-1-octene and heptadecafluoro-1-decene were obtained from Sigma Aldrich. These fluorinated olefins were subjected to solution-state fluorine-19 NMR analysis and very narrow bands were obtained as shown in Figure 17. The upper spectrum is of heptadecafluoro-1-decene and the lower spectrum is of tridecafluoro-1-octene. The chemical shifts are not exact as the bands were not referenced in this analysis; however one can qualitatively predict the purity of starting substrate. The upper spectrum has eight peaks representing the eight carbon atoms carrying fluorine in heptadecafluoro-1-decene and the lower spectrum has six narrow peaks for the six carbon atoms carrying fluorine in the tridecafluoro-1-octene. Hence, the fluorine -19 solution-state spectra ensures the purity of the olefins before its utilization in the hydrosilation procedure.

Afterwards, slurry state fluorine-19 NMR analyses were performed for silica hydride, TDF C8 and HDF C10 materials to check for any indication of fluorine related peaks. Figure 18 shows the slurry state fluorine-19 NMR spectrum of silica hydride material. There is no peak observed for silica hydride which is an expected result as silica hydride does not have any fluorine moiety. However, the slurry state fluorine-19 NMR spectra of tridecafluoro-1-octene as shown in Figure 19 and heptadecafluoro-1decene as shown in Figure 20 give one small but identifiable peak individually. Along with the reasoning that the fluorine-19 NMR can give a peak only if the sample material does possess any fluorine-containing moiety, it can be concluded that the TDF C8 and HDF C10 bonded phases do have fluorinated moieties attached to silica hydride 
stationary phases. These spectra further establish the success of the hydrosilation procedure confirmed by DRIFT spectroscopic and carbon elemental analyses.

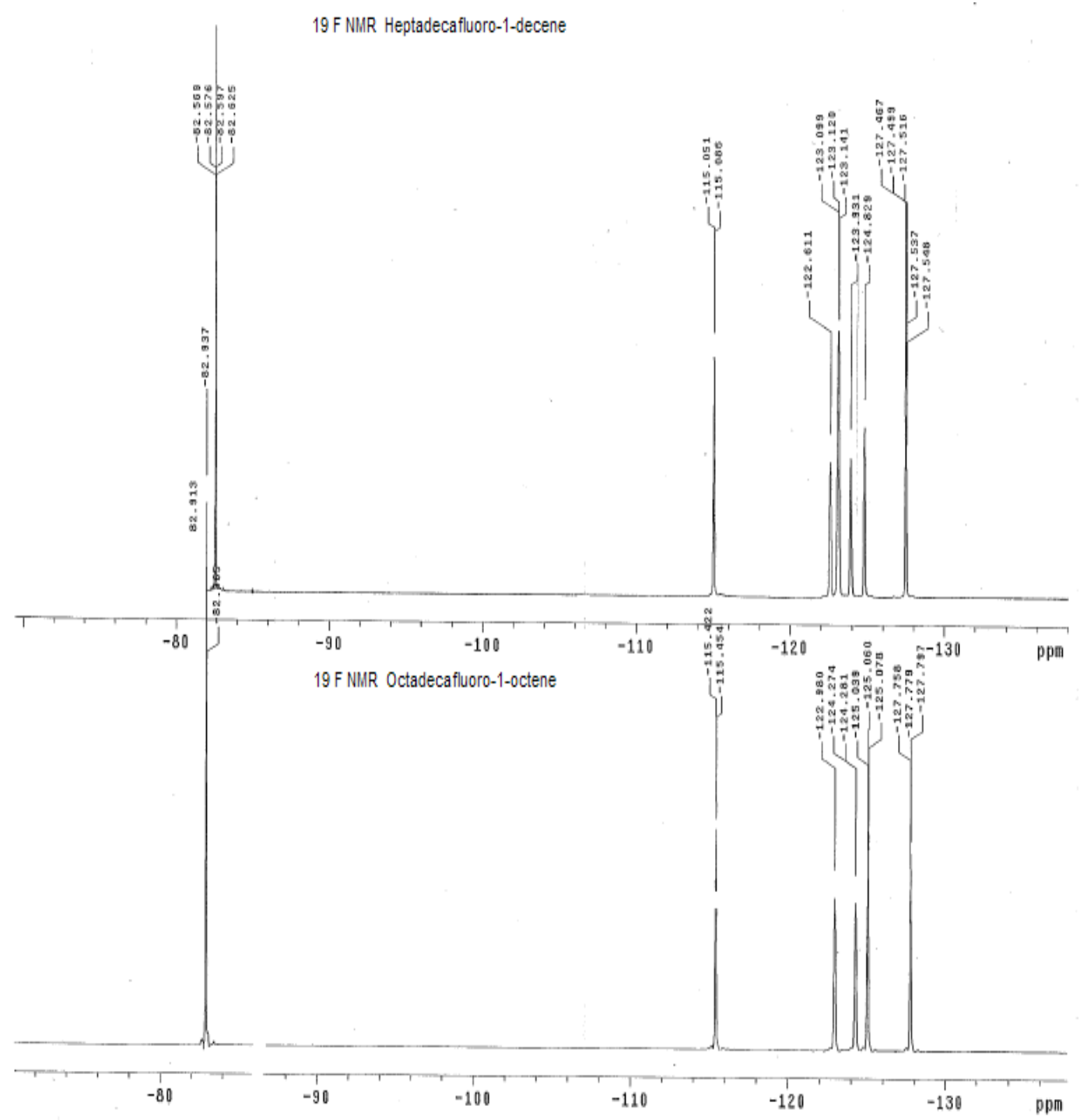

Figure 17. Fluorine-19 solution state NMR of heptadecafluoro-1-decene and tridecafluoro-1-octene olefins. 
19F NAR

Astrosil lived its in d-nethano

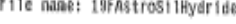

exp1 FLURImt

Sicint

SAKPLt
date Ju1 152008
80lvent C0.900
filent

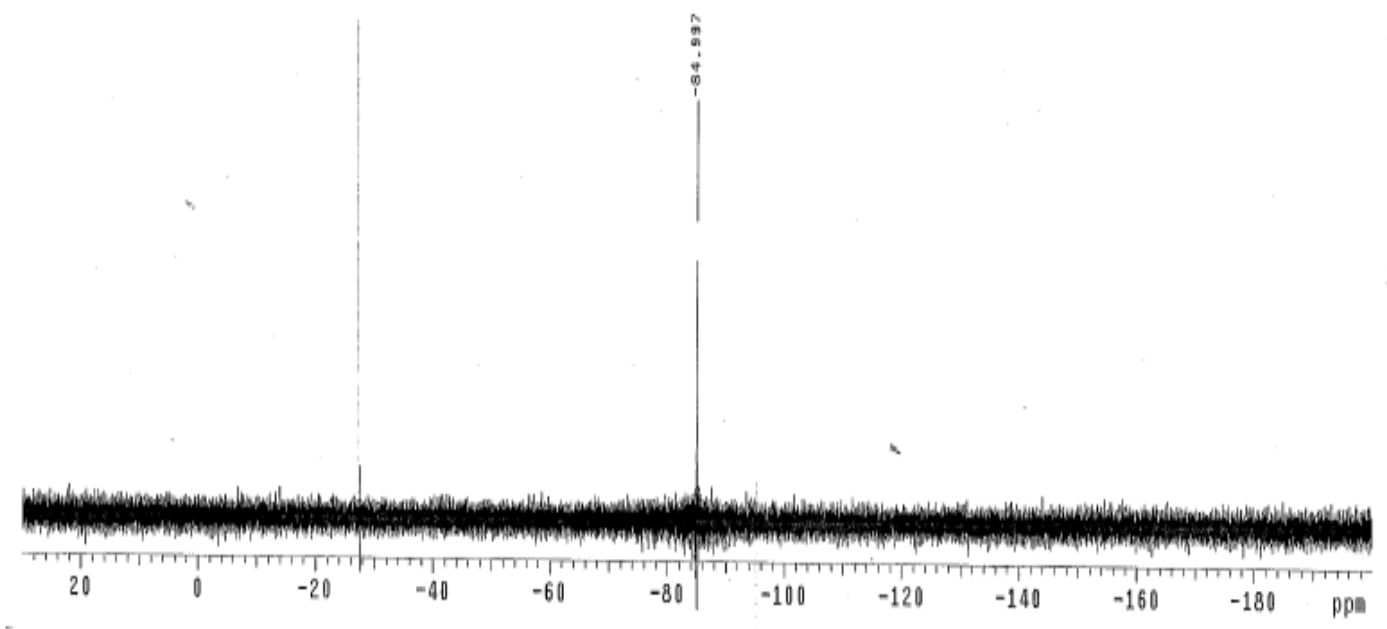

Figure 18. Fluorine-19 slurry state NMR of Astrosil silica hydride. 

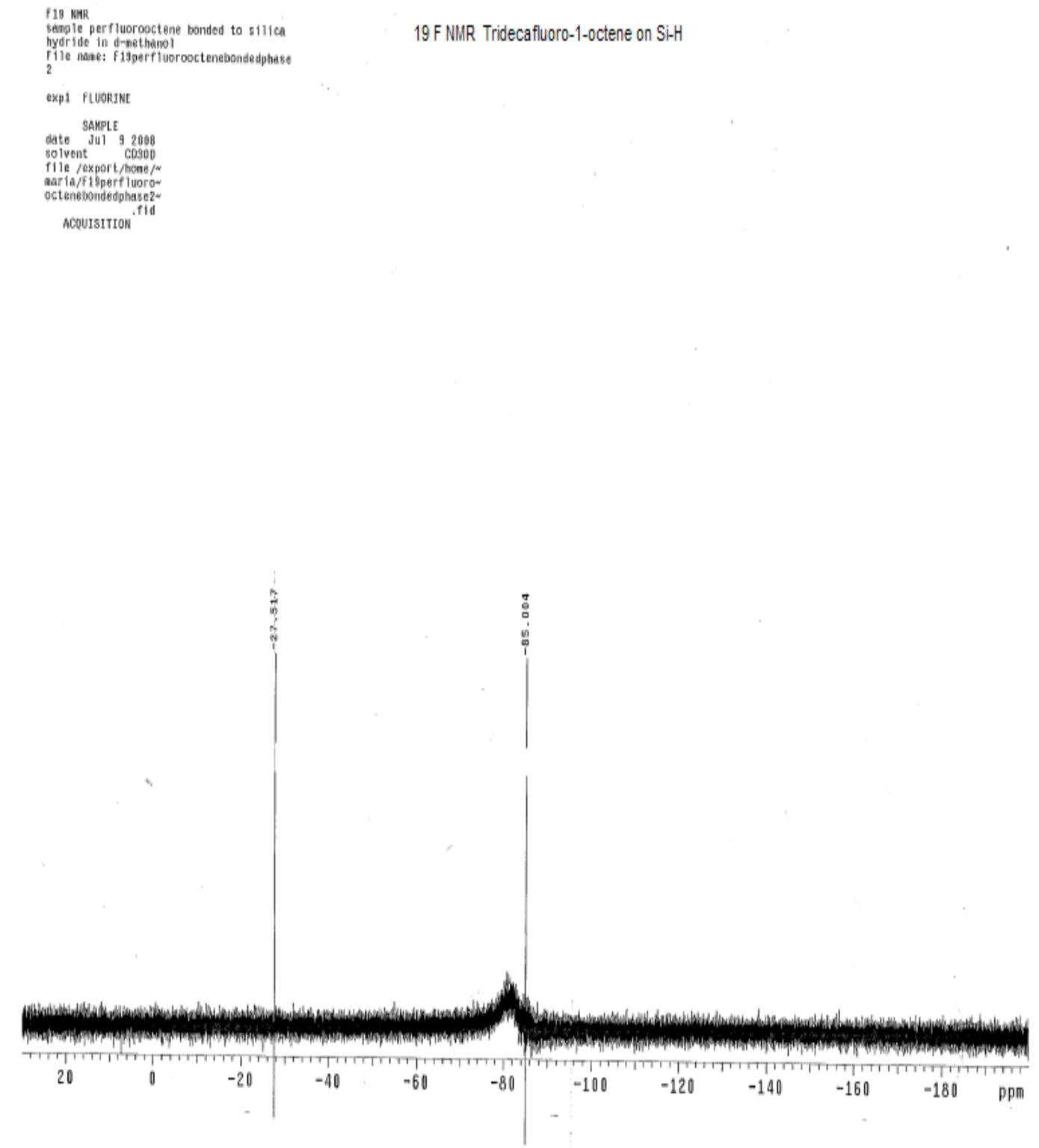

Figure 19. Fluorine-19 slurry state NMR of TDF C8. 
19F NMR Heptadecafluoro-1-decene on Si-H
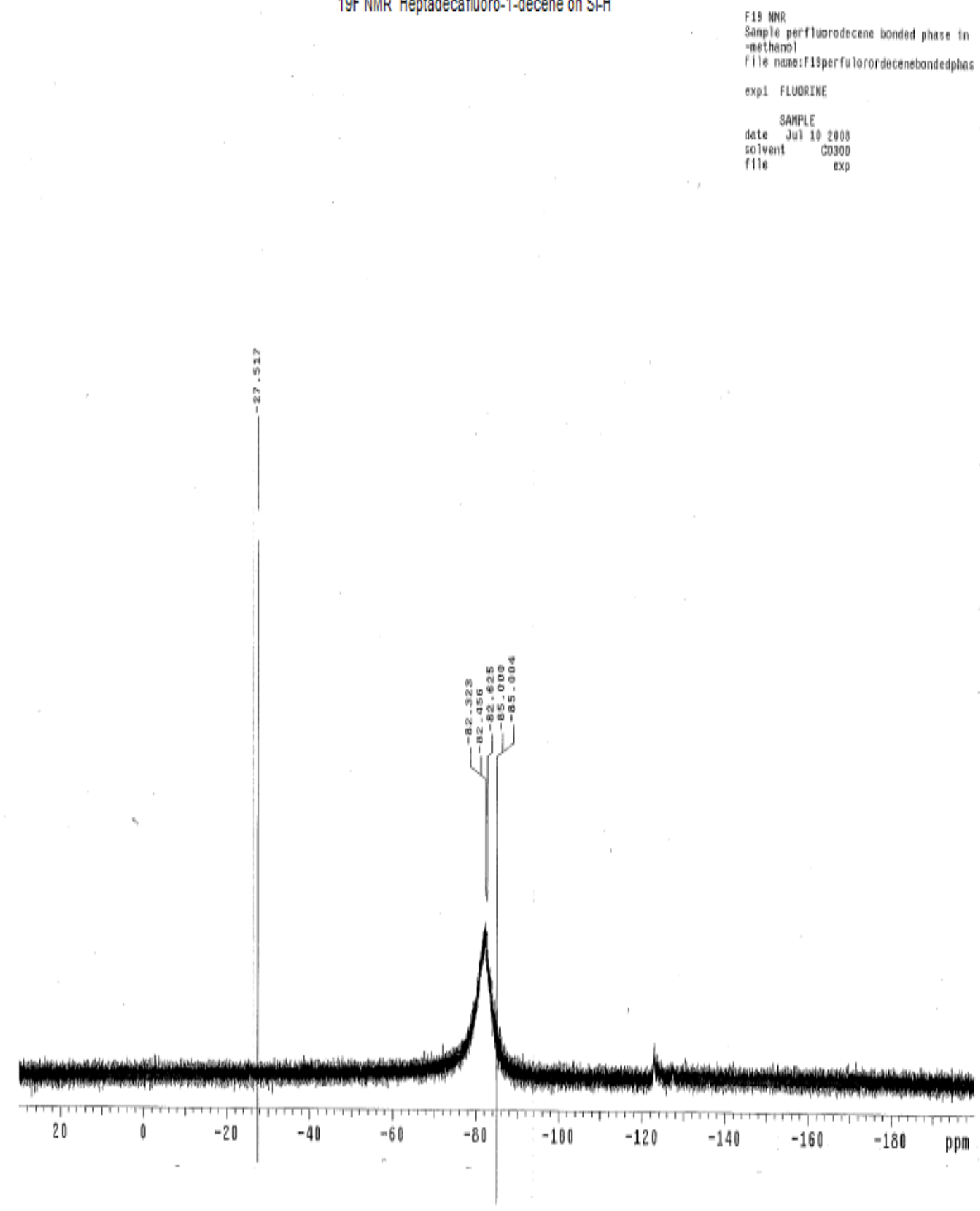

Figure 20. Fluorine-19 slurry state NMR of HDF C10. 


\section{HPLC Chromatographic Evaluation of Columns}

The main goal of the research was to establish the ANP retention behavior of the novel fluorinated stationary phases. The stainless steel columns with $100 \mathrm{~cm} \times 4.6 \mathrm{~mm}$ i.d. dimensions were packed with tridecafluoro-1-octene silica hydride (TDF C8) and heptadecafluoro-1-decene silica hydride (HDF C10) bonded phases. Since ANP retention is mainly observed for the polar or ionic molecules, different small polar compounds were analyzed on TDF C8 and HDF C10 columns using HPLC connected to ELSD, UV and MS detectors. Duplicates of each HPLC run were performed and documented for reference. The retention performance of these columns is discussed in the following sections.

\section{Retention Studies Using ELSD Detection}

First, the newly packed TDF C8 and HDF C10 columns were conditioned using binary solvents 100:0 acetonitrile:water for 1 hour followed by 90:10, 80:20, 70:30, 60:40, 50:50 acetonirtile:water for a half an hour each. During the optimization of the HPLC-ELSD experimental conditions mode, the flow rate of the HPLC runs was carefully selected to maintain the pressure of the pump between 500-800 psi. A few trials using higher sample concentrations than $1 \mathrm{mg} / \mathrm{mL}$ resulted in significant peak tailing and shorter retention times; hence a $1 \mathrm{mg} / \mathrm{mL}$ concentration was selected to make sample solutions for all amino acids, nucleobases, nucleosides, and ergogenic acids analyzed by ELSD. The flow rate was optimized to $0.5 \mathrm{~mL} / \mathrm{min}$ and the injection volume was set to $20 \mu \mathrm{L}$ for all of these solutes. For the ELSD detector, the flow rate of the nebulization gas (nitrogen gas) was set to 65 psi as described in the instrument specifications. 
Since the model 400 ELSD specifies a lower spray chamber temperature for aqueous mobile phases and higher drift tube temperature for a volatile mobile phase, the spray chamber temperature was kept at $25{ }^{\circ} \mathrm{C}$ and the drift tube temperature was optimized to $55^{0} \mathrm{C}$ for ANP retention studies. When the temperature of the drift tube was increased above $65{ }^{\circ} \mathrm{C}$, reduced intensity chromatographic peaks with splitting as the acetonitrile concentration increased was observed. However, in the drift tube temperature range of $55 \pm 5{ }^{0} \mathrm{C}$, no noticeable change in the peak shape or in the retention times was seen.

\subsection{Amino Acids}

To understand the ANP retention of different polarity amino acids; a pair of acidic, basic and neutral amino acids were selected for the retention analysis as shown in Table 5 . The sample solutions were prepared at $1 \mathrm{mg} / \mathrm{mL}$ using 50:50 acetonitrile:water premixed with $0.1 \%$ Formic acid (FA). The arginine and histidine amino acids were readily dissolved, whereas the aspartic acid and tyrosine were hard to dissolve to make $1 \mathrm{mg} / \mathrm{mL}$ sample solutions. Before starting each run, columns were conditioned for 15-20 minutes using 50:50 acetonitrile:water premixed with $0.1 \%$ FA mobile phases. Six different polarity amino acids were run on both columns at different concentrations of acetonitirle.

Table 5 shows the retention times of amino acids and the percentage composition of acetonitirle:water which is the ANP binary solvent system for polar solutes analyzed on HDF C10 column. As the concentration of acetonitrile increases from $50 \%$ to $90 \%$, the retention times for all the amino acids increases gradually. The graphical representation 
of ANP retention is plotted as $\%$ acetonitirle vs. retention time for each amino acid which is referred to as a retention map. Figure 21 shows the retention maps of all the amino acids analyzed on HDF C10 column along with their chemical structures. The basic arginine and histidine amino acids are the most retained; followed by neutral polar tyrosine and phenylalanine, and acidic glutamic acid and aspartic acid. As the concentration of acetonitrile increases from $50 \%$ to $90 \%$, the amino acids show significant ANP retention on the HDF $\mathrm{C} 10$ column. The arginine and aspartic acid amino acids were also run to check their retention for reversed phase conditions of 10:90, 20:80, 30:70 and 40:60 acetonitrile:water mobile phases but for each combination they eluted at the void volume and showed no retention with acetonitrile lower than $50 \%$. They start retaining as the $\%$ of acetonitrile increases from $50 \%$ and above. This result establishes the ANP behavior of the HDF C10 column.

Table 6 shows the ANP retention time data of the amino acids run on the TDF C8 column. Similar ANP retention for each amino acid has been observed on this column as well. Figure 22 shows the retention maps of amino acids analyzed on the TDF C8. The amino acids are retained on the TDF C8 as the concentration of acetonitrile increased from $50 \%$ to $90 \%$ showing the ANP behavior of the column. This column also has shown similar higher retention for basic amino acids followed by neutral and acidic amino acids. The comparison of retention maps of polar neutral tyrosine and phenylalanine amino acids on TDF C8 is plotted in Figure 23. Since the tyrosine has an additional $-\mathrm{OH}$ polar functional group it shows higher ANP retention compared to 
phenylalanine as the acetonitrile concentration increases. Thus, this data supports the idea that as the polarity of the solute increases, greater ANP retention is obtained.

Table 5. Retention times of amino acids on the HDF C10 column using ELSD

\begin{tabular}{|c|c|c|c|c|c|c|c|}
\hline \multicolumn{2}{|c|}{$\begin{array}{c}\text { Concentration } \\
\text { (\%) }\end{array}$} & \multicolumn{5}{c|}{$\begin{array}{r}\text { Retention time } \\
\text { (min) }\end{array}$} \\
\hline $\begin{array}{c}\text { Acetonitrile } \\
\text { with } \\
0.1 \% \text { FA }\end{array}$ & $\begin{array}{c}\text { DI water } \\
\text { with } \\
0.1 \% \text { FA }\end{array}$ & $\begin{array}{c}\text { ARG } \\
\text { (basic) }\end{array}$ & $\begin{array}{c}\text { HIS } \\
\text { (basic) }\end{array}$ & $\begin{array}{c}\text { ASP } \\
\text { (acidic) }\end{array}$ & $\begin{array}{c}\text { GLU } \\
\text { (acidic) }\end{array}$ & $\begin{array}{c}\text { PHE } \\
\text { (neutral) }\end{array}$ & $\begin{array}{c}\text { TYR } \\
\text { (neutral) } \\
\text { (polar) }\end{array}$ \\
\hline 50 & 50 & 3.384 & 3.214 & 2.544 & 2.626 & 3.043 & 2.841 \\
\hline 60 & 40 & 4.213 & 4.006 & 2.763 & 2.916 & 3.183 & 3.205 \\
\hline 70 & 30 & 6.430 & 6.646 & 3.385 & 3.666 & 3.848 & 4.143 \\
\hline 75 & 25 & 9.484 & 10.020 & 4.094 & 4.431 & 4.480 & 5.094 \\
\hline 80 & 10 & - & - & 16.315 & 21.773 & 19.680 & 25.916 \\
\hline 85 & 15 & - & - & 8.183 & 8.937 & 9.020 & 11.482 \\
\hline
\end{tabular}

\begin{tabular}{|l|l|}
\hline $\mathrm{ARG}=$ Arginine & GLU $=$ Glutamic acid \\
\hline HIS $=$ Histidine & PHE $=$ Phenylalanine \\
\hline ASP $=$ Aspartic acid & TYR $=$ Tyrosine \\
\hline
\end{tabular}




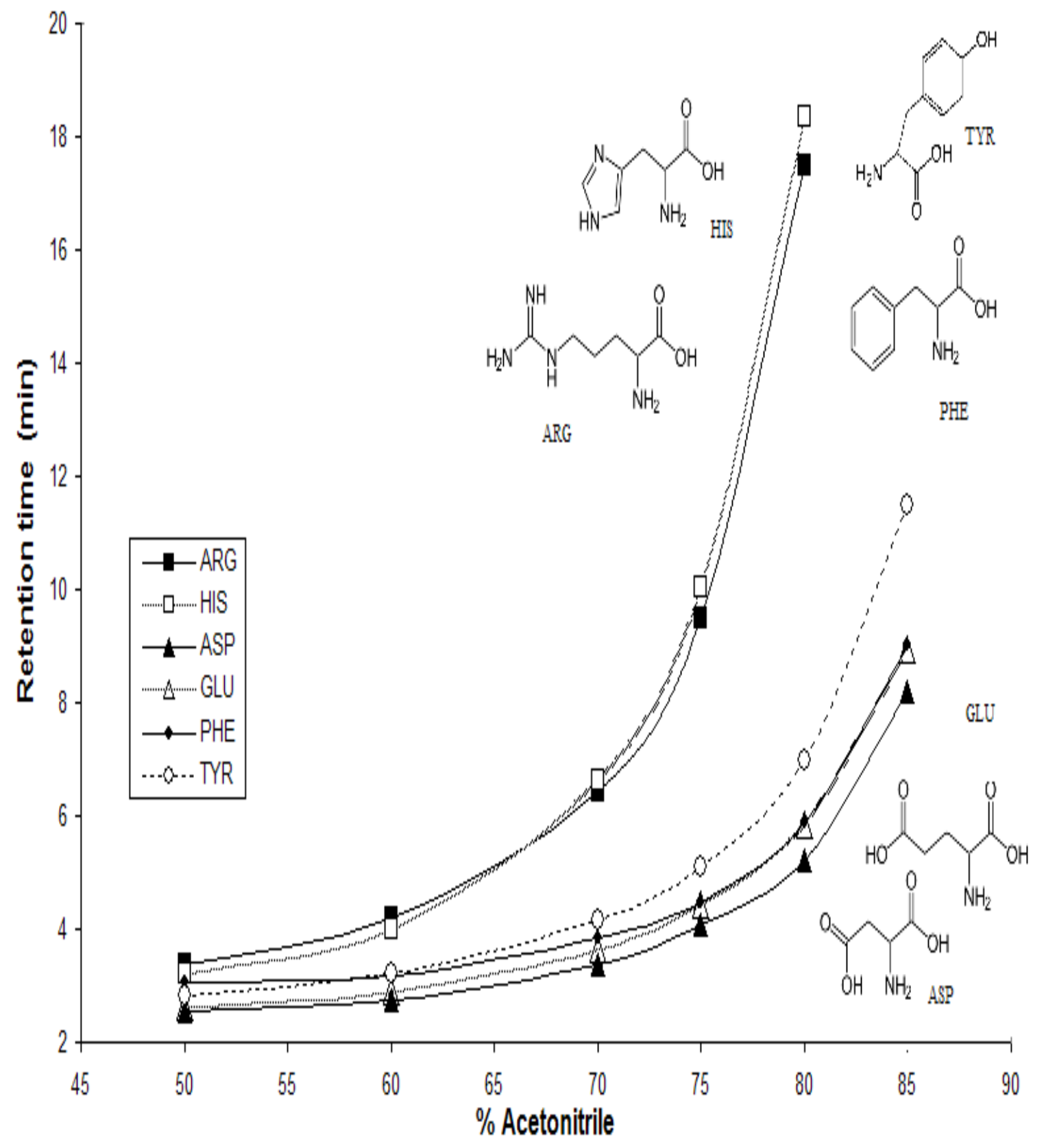

Figure 21. Retention map of amino acids on the HDF C10 column using ELSD. 
Table 6. Retention times of amino acids on the TDF C8 column using ELSD

\begin{tabular}{|c|c|c|c|c|c|c|c|}
\hline \multicolumn{2}{|c|}{$\begin{array}{c}\text { Concentration } \\
\text { (\%) }\end{array}$} & \multicolumn{5}{|c|}{$\begin{array}{c}\text { Retention time } \\
\text { (min) }\end{array}$} \\
\hline $\begin{array}{c}\text { Acetonitrile } \\
\text { with } \\
0.1 \% \text { FA }\end{array}$ & $\begin{array}{c}\text { DI water } \\
\text { with } \\
0.1 \% \text { FA }\end{array}$ & $\begin{array}{c}\text { ARG } \\
\text { (basic) }\end{array}$ & $\begin{array}{c}\text { HIS } \\
\text { (basic) }\end{array}$ & $\begin{array}{c}\text { ASP } \\
\text { (acidic) }\end{array}$ & $\begin{array}{c}\text { GLU } \\
\text { (acidic) }\end{array}$ & $\begin{array}{c}\text { PHE } \\
\text { (neutral) }\end{array}$ & $\begin{array}{c}\text { TYR } \\
\text { (neutral) } \\
\text { (polar) }\end{array}$ \\
\hline 50 & 50 & 3.276 & 3.261 & 2.456 & 2.539 & 2.940 & 2.964 \\
\hline 60 & 40 & 4.170 & 4.362 & 2.685 & 2.814 & 3.209 & 3.456 \\
\hline 70 & 30 & 6.940 & 7.416 & 3.366 & 3.578 & 3.885 & 4.531 \\
\hline 75 & 25 & 10.057 & 11.012 & 3.986 & 4.458 & 4.796 & 5.676 \\
\hline 80 & 20 & 18.186 & 21.884 & 5.208 & 5.856 & 6.344 & 8.022 \\
\hline 90 & 10 & - & - & 16.688 & 21.556 & 16.963 & 28.961 \\
\hline
\end{tabular}




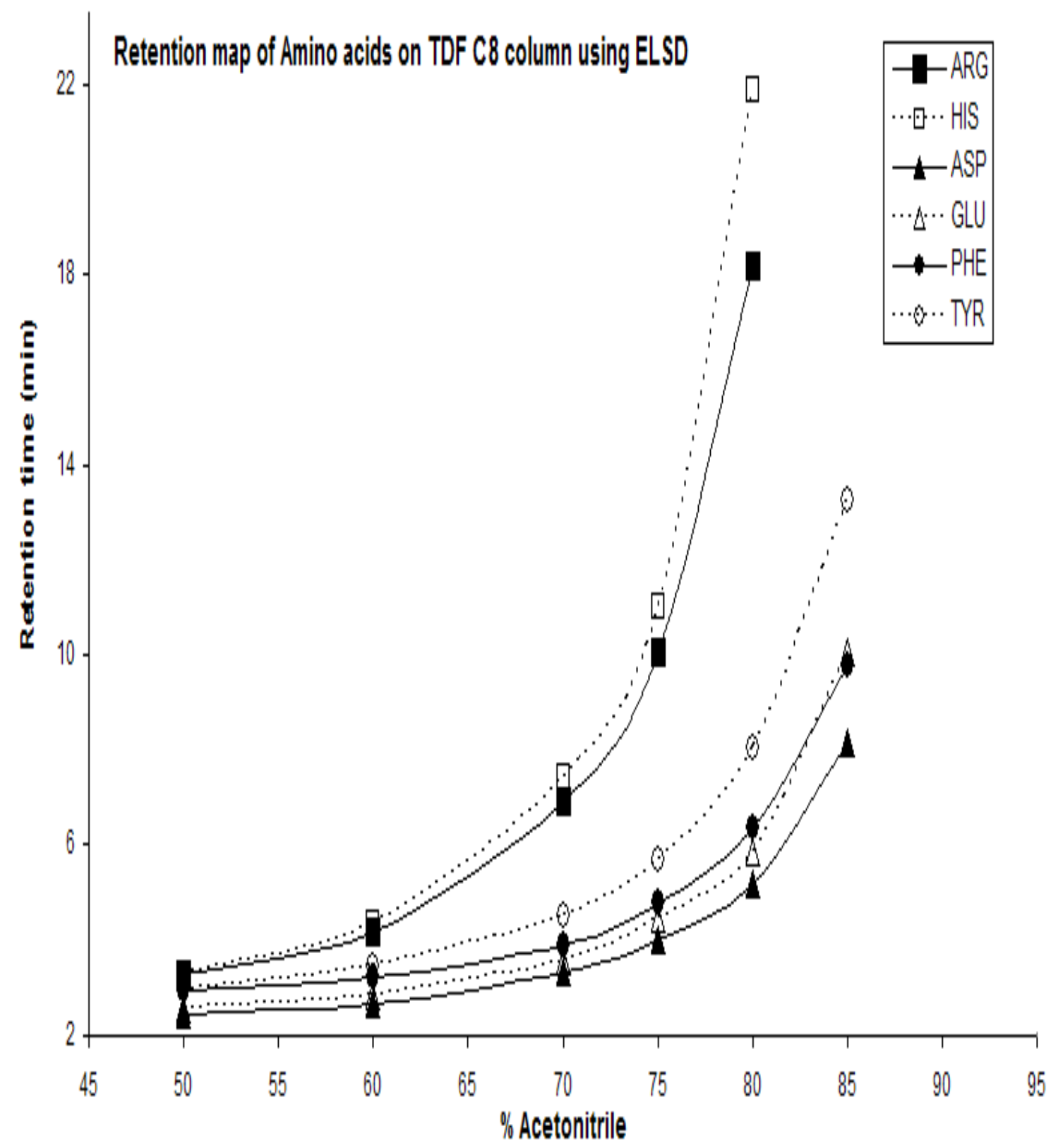

Figure 22. Retention map of amino acids on the TDF C8 column using ELSD. 


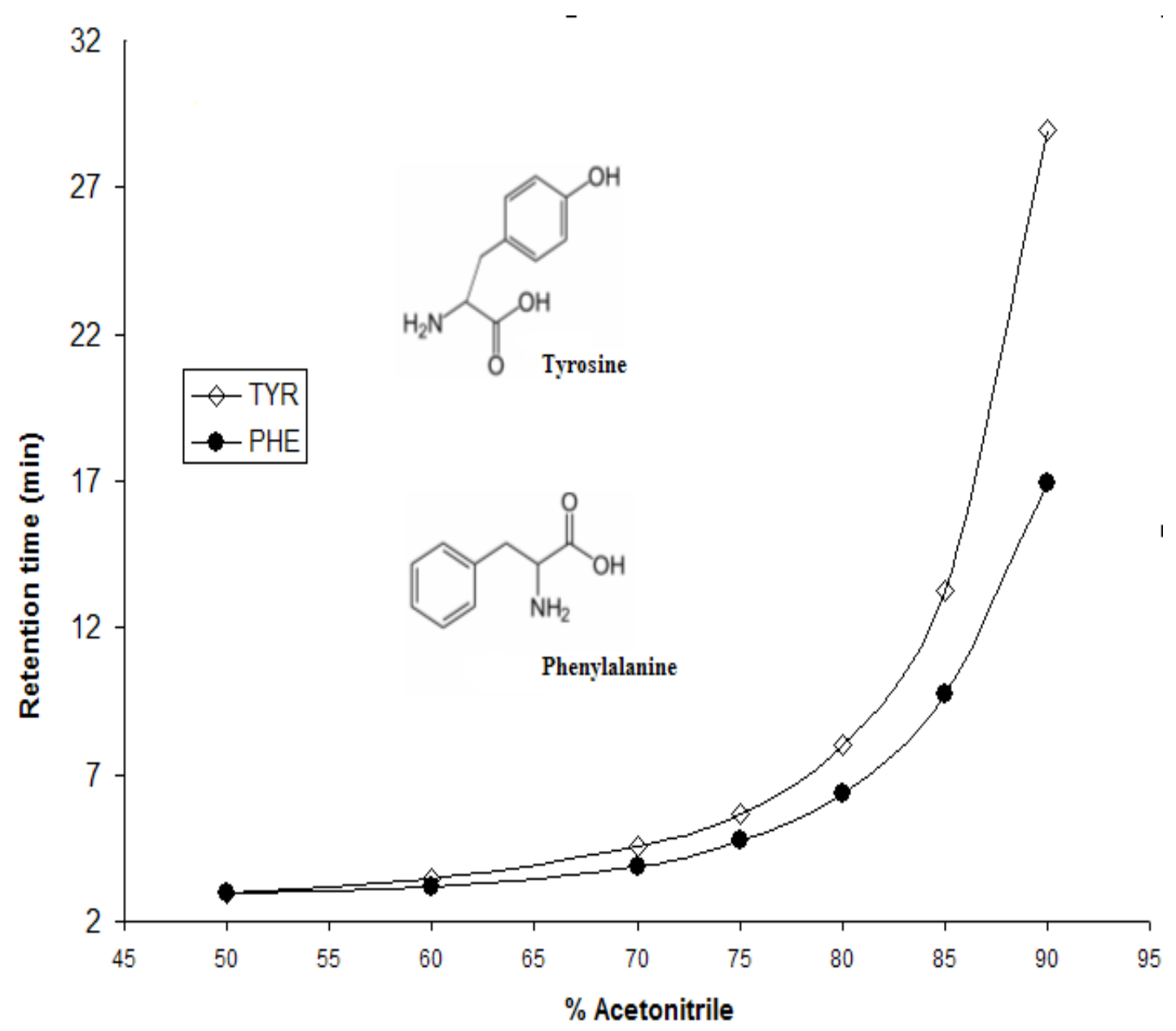

Figure 23. Comparison of retention maps of tyrosine and phenylalanine on the TDF C8 using ELSD.

When the ANP retention of these amino acids on two columns (TDF C8 and HDF C10) is compared, several interesting observations can be made. One can easily distinguish the retention time differences of the strongly basic arginine and histidine, and neutral polar tyrosine and phenylalanine on TDF C 8 and HDF C10 as shown in Table 5 and Table 6 . The TDF C 8 column retained arginine, histidine and tyrosine more than the HDF C10 column because as determined in the carbon elemental analysis, the TDF C8 
column has somewhat more of the fluorinated stationary phase responsible for retention of the polar amino acids. Phenylalanine being less polar, retains more on the HDF C10 which has more nonpolar silica hydride layer exposed which is responsible for hydrophobic retention. The graphical presentation of this fact can be seen as a comparison of retention maps on the two columns as shown in Figures 24a, b, c, and d for arginine, histidine, tyrosine, and phenylalanine respectively.

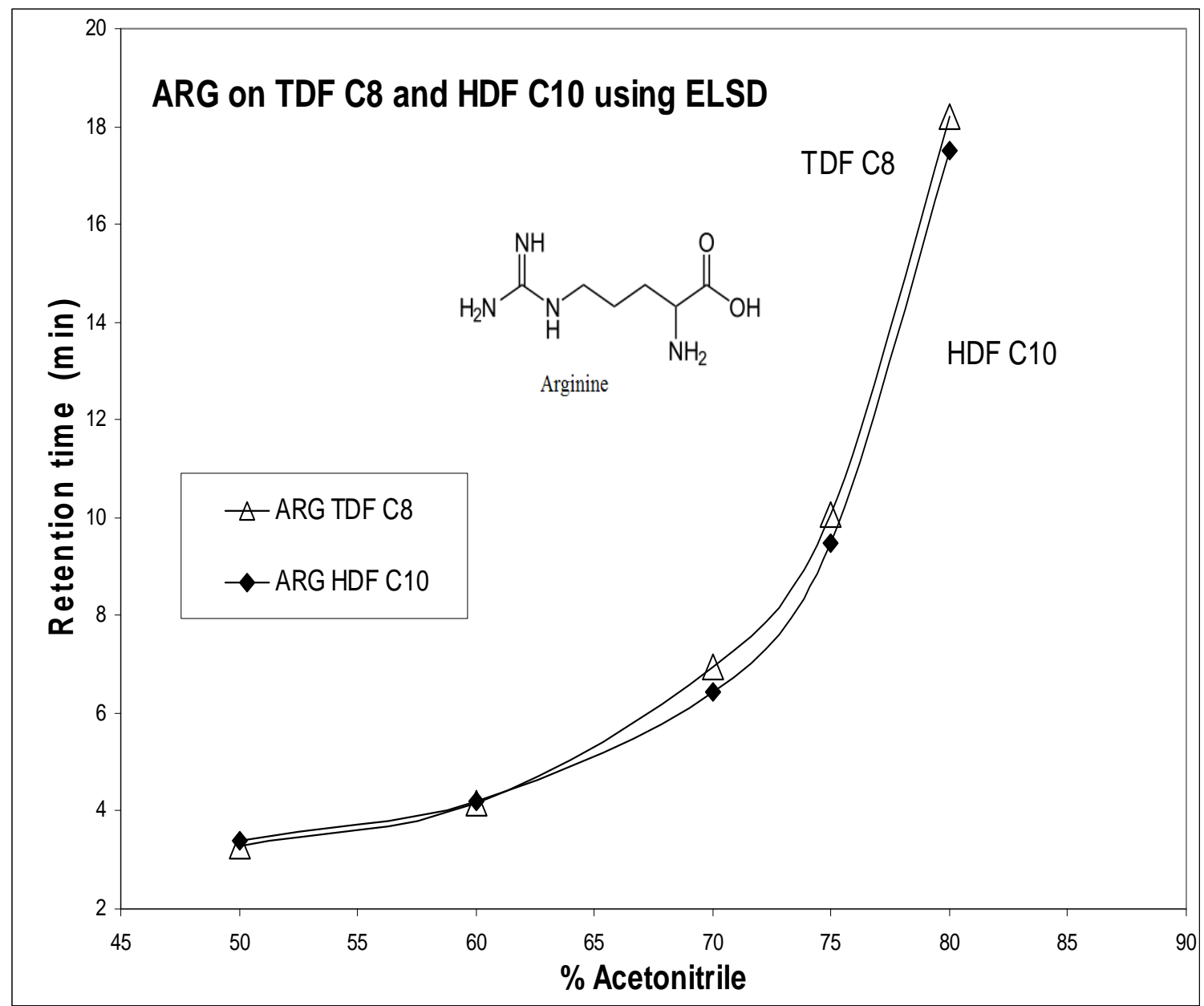

Figure 24a. Comparison of retention maps of arginine analyzed on two columns. 


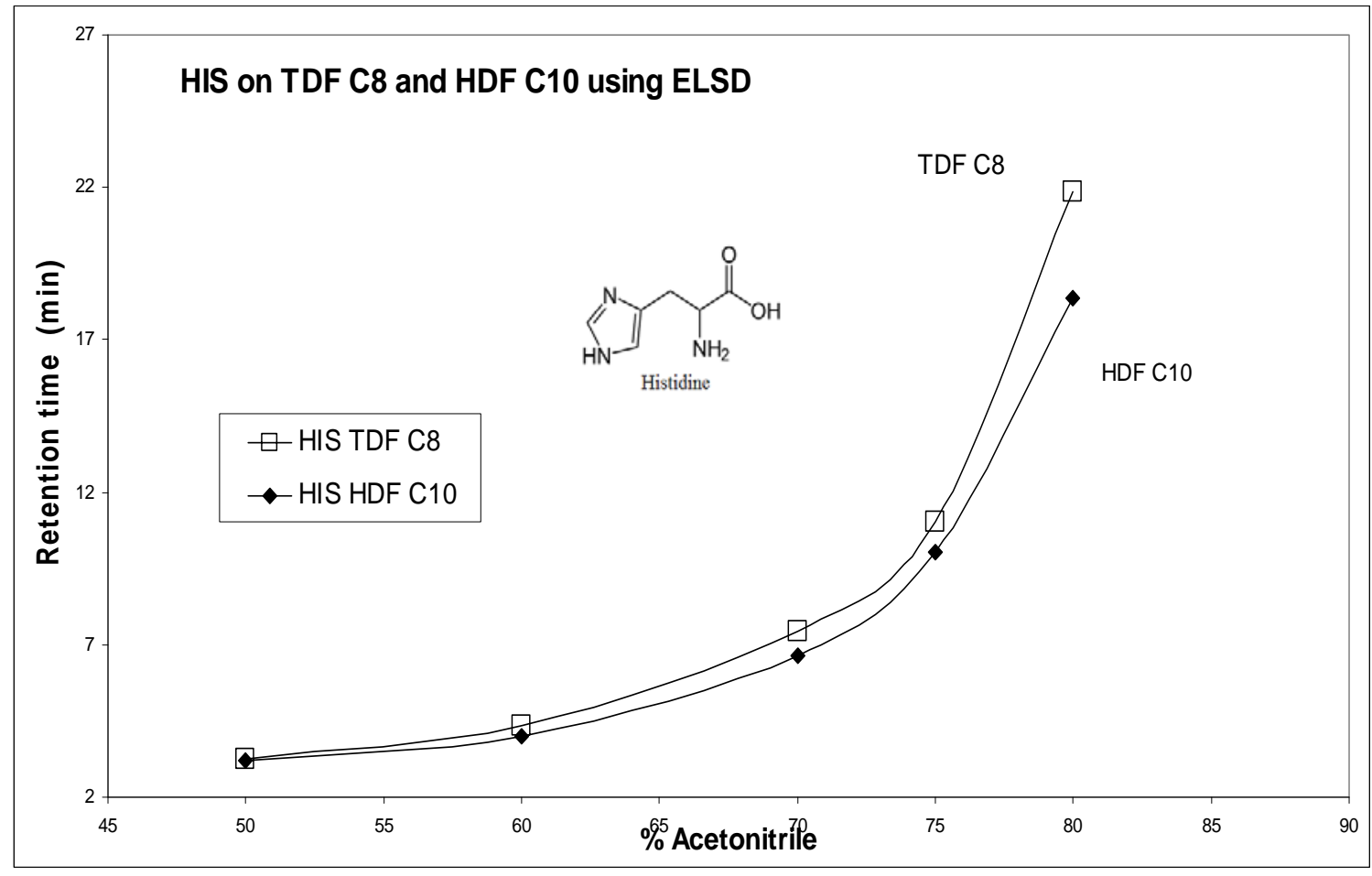

Figure 24b. Comparison of retention maps of histidine analyzed on two columns.

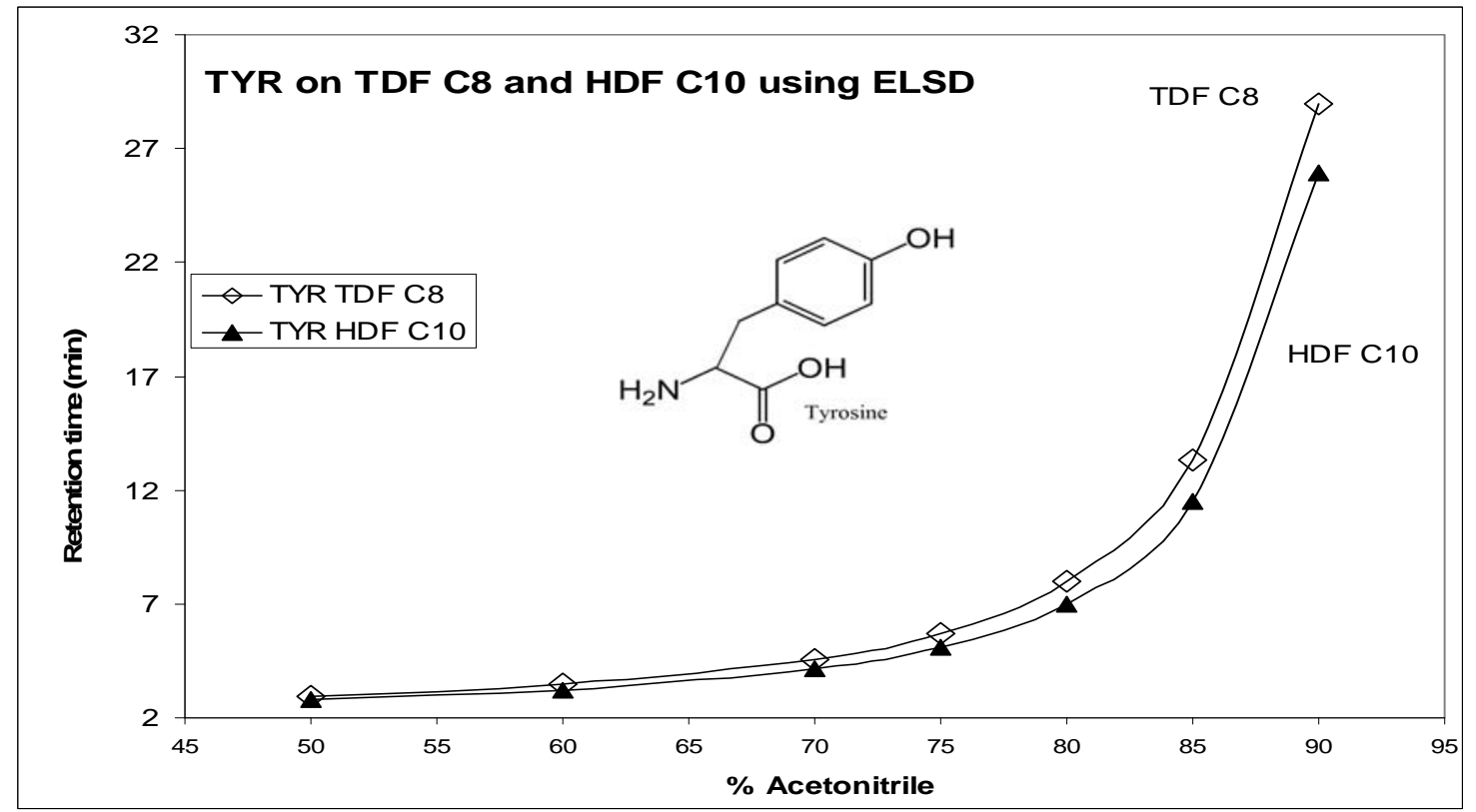

Figure 24c. Comparison of retention maps of tyrosine analyzed on two columns. 


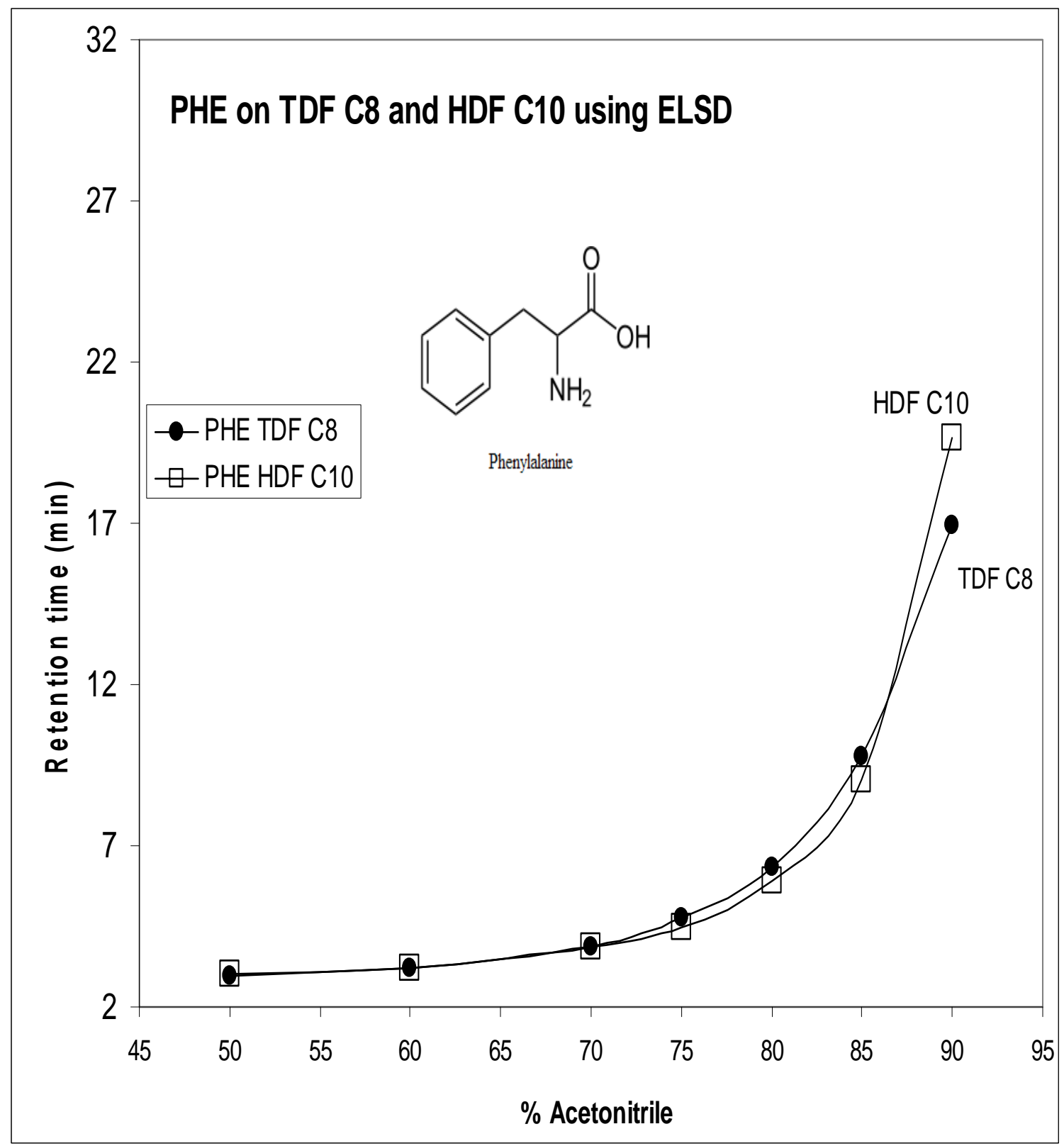

Figure 24d. Comparison of retention maps of phenylalanine on two columns.

In general, as for each mobile phase composition, different amino acids show different retention times on a single column, they could be separated from one another using gradient development. Since many proteins and peptides have amino acids in their 
building block units, it is possible that they can be separated on these columns based on the retention data of amino acids reported here.

\subsubsection{Reproducibility Study (\% RSD)}

The reproducibility study was conducted on both columns for arginine and tyrosine using 70:30 acetonitrile:water mobile phase combination premixed with $0.1 \%$ FA. As shown in Table 7, ten consecutive injections of arginine and tyrosine were performed and the retention times of each on both TDF C 8 and HDF C10 columns were recorded to calculate $\%$ RSD values. The range of \% RSD values obtained is $0.19-0.77$ which is remarkable since values less than 1 are difficult to obtain even for reversed-phase retention. Hence, these columns promise a very high degree of precision for ANP retention data.

\subsection{Nucleobases}

The nucleobases cytosine, guanine and uracil were analyzed under ANP solvent conditions on both columns. Table 8 and Table 9 show the retention times at various $\%$ concentrations of acetonitrile:water with $0.1 \%$ FA. Cytosine was retained very strongly as the concentration of the acetonitrile increased. Guanine and uracil also had considerable retention thus confirming the ANP behavior of this class of compounds. Guanine was difficult to dissolve. It could only be dissolved in a $200 \mu \mathrm{L} \mathrm{HCl}$ solution containing 50:50 acetonitrile:water premixed with $0.1 \%$ FA to make $1 \mathrm{mg} / \mathrm{mL}$ sample solution. It is important to note that uracil is a highly polar compound and is generally used to determine the void volume in reversed phase chromatography. However, in the 
ANP mode, it was retained on both the TDF C8 and HDF C10 columns with distinguishable retention time differences as the \% acetonitrile increases.

Table 7. Reproducibility for 10 injections of two amino acids using ELSD

\begin{tabular}{|c|c|c|c|c|}
\hline \multirow{3}{*}{$\begin{array}{l}\text { Number of } \\
\text { Injections }\end{array}$} & \multicolumn{4}{|c|}{ Retention Time (min.) } \\
\hline & $\begin{array}{c}\text { Aceton } \\
\text { (premixe }\end{array}$ & $\begin{array}{l}\text { DI Water } \\
0.1 \% \text { FA) }\end{array}$ & $\begin{array}{l}\text { Aceton } \\
\text { (premix }\end{array}$ & $\begin{array}{l}\text { e } \\
\text { I Water } \\
0.1 \% \text { FA) }\end{array}$ \\
\hline & TDF C8 & HDF C10 & TDF C8 & HDF C10 \\
\hline 1 & 6.93 & 6.39 & 4.38 & 4.15 \\
\hline 2 & 7.04 & 6.38 & 4.38 & 4.14 \\
\hline 3 & 6.93 & 6.42 & 4.36 & 4.14 \\
\hline 4 & 6.94 & 6.45 & 4.37 & 4.13 \\
\hline 5 & 6.86 & 6.44 & 4.36 & 4.14 \\
\hline 6 & 6.94 & 6.48 & 4.33 & 4.14 \\
\hline 7 & 6.84 & 6.46 & 4.33 & 4.15 \\
\hline 8 & 6.94 & 6.36 & 4.38 & 4.14 \\
\hline 9 & 6.94 & 6.38 & 4.35 & 4.16 \\
\hline 10 & 6.91 & 6.46 & 4.32 & 4.15 \\
\hline$\%$ RSD & 0.77 & 0.66 & 0.49 & 0.19 \\
\hline
\end{tabular}


Table 8. Retention times of nucleobases on the TDF C8 column using ELSD

\begin{tabular}{|c|c|c|c|c|}
\hline \multicolumn{2}{|c|}{$\begin{array}{c}\text { Concentration } \\
\text { (\%) }\end{array}$} & \multicolumn{3}{|c|}{$\begin{array}{l}\text { Retention time } \\
\text { (min) }\end{array}$} \\
\hline $\begin{array}{c}\text { Acetonitrile } \\
\text { with } \\
0.1 \% \text { FA }\end{array}$ & $\begin{array}{c}\text { DI Water } \\
\text { with } \\
0.1 \% \mathrm{FA}\end{array}$ & Cytosine & Guanine & Uracil \\
\hline 50 & 50 & 3.30 & 2.24 & 2.42 \\
\hline 60 & 40 & 4.01 & 2.33 & 2.45 \\
\hline 70 & 30 & 5.50 & 2.44 & 2.55 \\
\hline 75 & 25 & 6.86 & - & - \\
\hline 80 & 20 & 9.24 & 2.85 & 2.74 \\
\hline 85 & 15 & 14.48 & 3.30 & 2.87 \\
\hline 90 & 10 & - & 4.50 & 3.07 \\
\hline 95 & 05 & - & - & 3.22 \\
\hline
\end{tabular}

Figure 25 and Figure 26 represent the characteristic ANP retention maps of these three nucleobases on the TDF C8 and HDF C10 columns respectively showing very good ANP retention. Although cytosine was retained on both columns, the combination of 
70:30 and 75:25 acetonitrile:water on HDF C10 column resulted in unusually similar retention times of 6.10 minutes and 6.13 minutes respectively. This partly constant retention of cytosine is observed only on the HDF C10 column which may be the result of the characteristic behavior for the fluorinated C10 type of stationary phase. It is worthwhile to state that the retention times for $70 \%$ and $75 \%$ acetonitrile were reproducible and cytosine in general showed highly efficient symmetric chromatographic peaks on both columns.

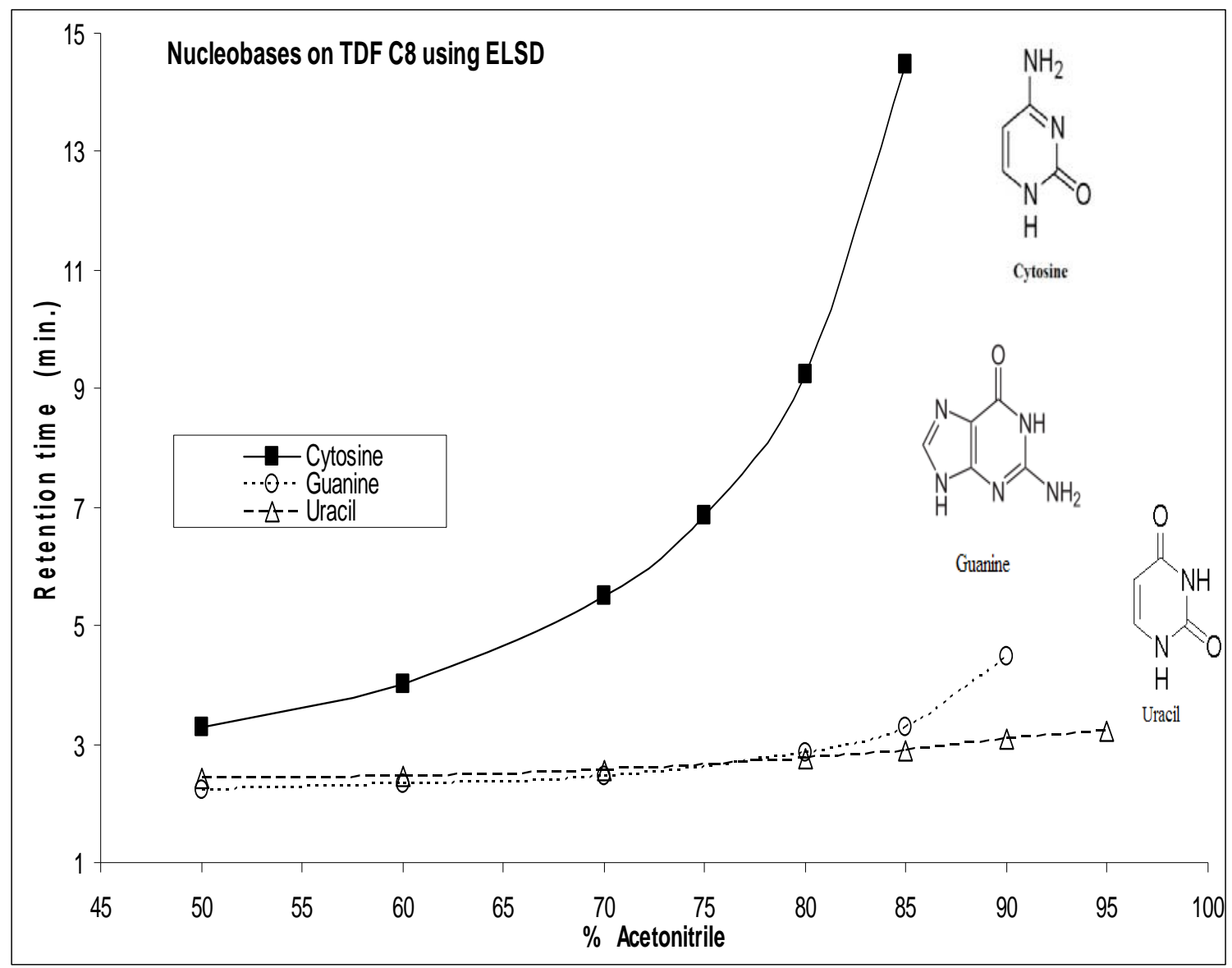

Figure 25. Retention map of nucleobases on the TDF C8 column using ELSD. 
Table 9. Retention times of nucleobases on the HDF C10 column using ELSD

\begin{tabular}{|c|c|c|c|c|}
\hline \multicolumn{2}{|c|}{$\begin{array}{c}\text { Concentration } \\
\text { (\%) }\end{array}$} & \multicolumn{3}{|c|}{$\begin{array}{l}\text { Retention time } \\
\text { (min) }\end{array}$} \\
\hline $\begin{array}{c}\text { Acetonitrile } \\
\text { with } \\
0.1 \% \text { FA }\end{array}$ & $\begin{array}{c}\text { DI Water } \\
\text { with } \\
0.1 \% \text { FA }\end{array}$ & Cytosine & Guanine & Uracil \\
\hline 50 & 50 & 3.27 & 2.33 & 2.46 \\
\hline 60 & 40 & 3.83 & 2.44 & 2.51 \\
\hline 70 & 30 & 6.10 & 2.70 & 2.61 \\
\hline 75 & 25 & 6.13 & - & - \\
\hline 80 & 20 & 8.00 & 3.24 & 2.79 \\
\hline 85 & 15 & 12.07 & 3.89 & 2.86 \\
\hline 90 & 10 & 23.03 & 5.58 & 3.05 \\
\hline 95 & 05 & - & - & 3.26 \\
\hline
\end{tabular}




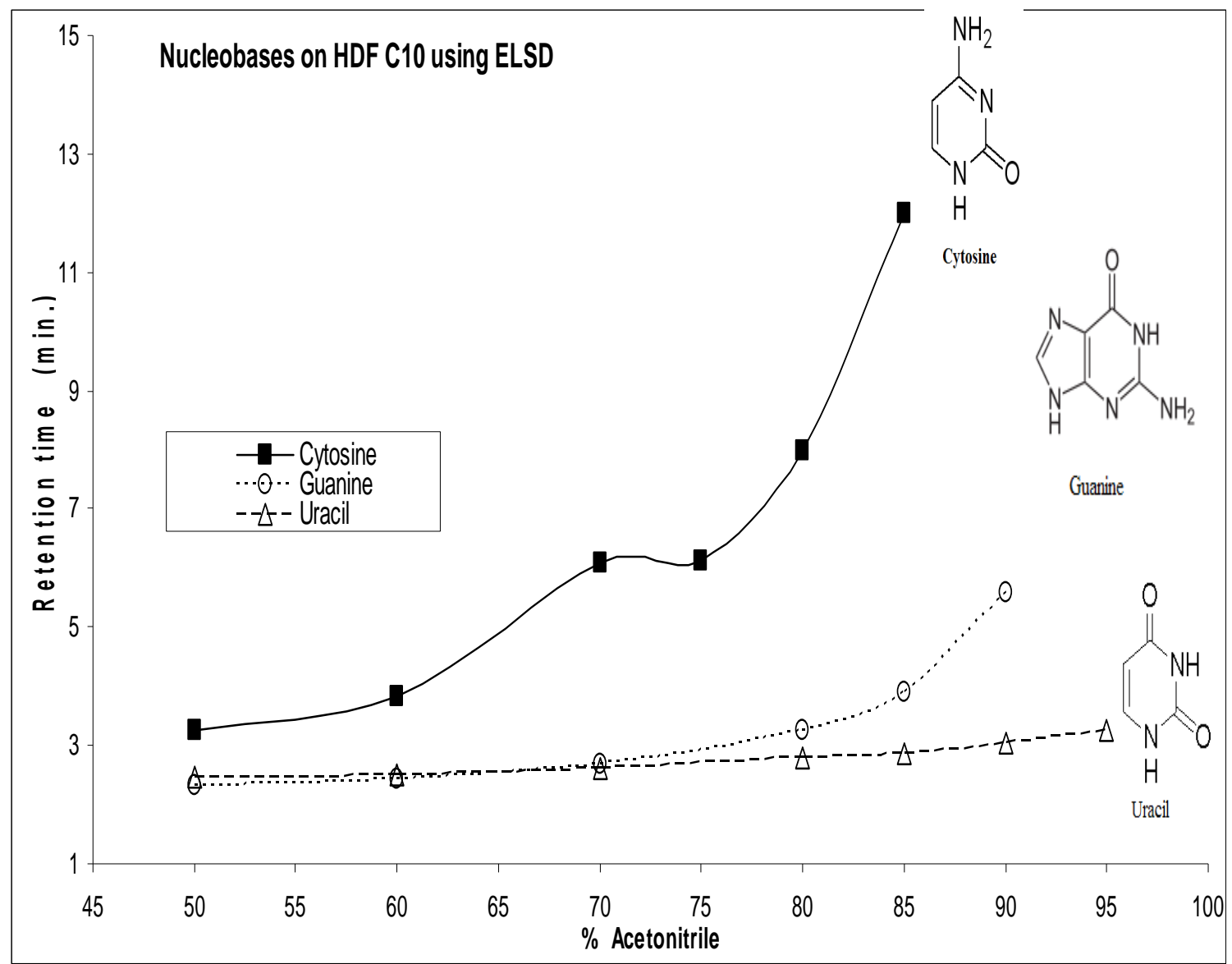

Figure 26. Retention map of nucleobases on HDF C10 column using ELSD.

\subsection{Nucleosides}

Table 10 and Table 11 report the retention times of adenosine and thymidine nucleosides as the concentration of acetonitrile increases from $50 \%$ to $90 \%$. In comparing nucleoside ANP retention on the TDF C8 and HDF C10 columns, similar retention maps of these compounds are obtained on both columns as presented in Figure 27 and Figure 28 respectively. Therefore, in the case of nucleosides one can estimate comparable contributions of the fluorinated moiety and the silica hydride surface for retaining these compounds. However, when comparing the two nucleosides, adenosine 
with a purine ring shows higher ANP retention than thymidine with a pyrimidine ring.

Hence, this observation also supports the fact that a higher polarity of the solute results in stronger ANP retention.

Table 10. Retention times of nucleosides on the TDF C8 column using ELSD

\begin{tabular}{|c|c|c|c|}
\hline \multicolumn{2}{|c|}{$\begin{array}{c}\text { Concentration } \\
(\%)\end{array}$} & \multicolumn{2}{|c|}{$\begin{array}{c}\text { Retention time } \\
\text { (min) }\end{array}$} \\
\hline $\begin{array}{c}\text { Acetonitrile } \\
\text { with } \\
0.1 \% \text { FA }\end{array}$ & $\begin{array}{c}\text { DI Water } \\
\text { with } \\
0.1 \% \text { FA }\end{array}$ & Adenosine & Thymidine \\
\hline 50 & 50 & 2.99 & 2.35 \\
\hline 60 & 40 & 3.26 & 2.37 \\
\hline 70 & 30 & 3.72 & 2.50 \\
\hline 80 & 20 & 4.93 & 2.70 \\
\hline 85 & 15 & 6.34 & 2.88 \\
\hline 90 & 10 & 8.97 & 3.12 \\
\hline 95 & 05 & - & - \\
\hline
\end{tabular}




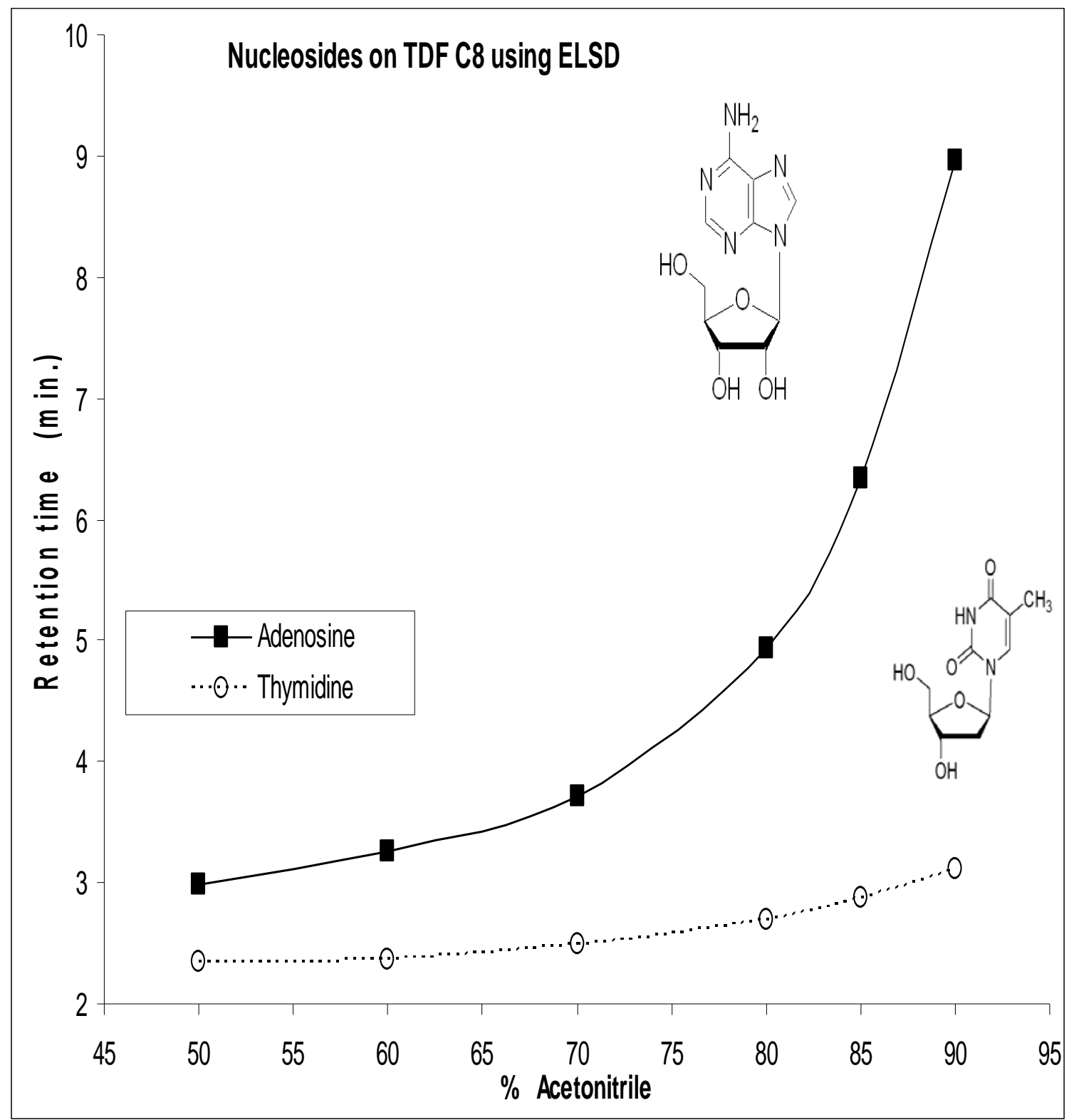

Figure 27. Retention maps of nucleosides on the TDF C8 column using ELSD. 
Table 11. Retention times of nucleosides on the HDF C10 column using ELSD

\begin{tabular}{|c|c|c|c|}
\hline \multicolumn{2}{|c|}{$\begin{array}{c}\text { Concentration } \\
(\%)\end{array}$} & \multicolumn{2}{|c|}{$\begin{array}{c}\text { Retention time } \\
\text { (min) }\end{array}$} \\
\hline $\begin{array}{c}\text { Acetonitrile } \\
\text { with } \\
0.1 \% \mathrm{FA}\end{array}$ & $\begin{array}{c}\text { DI Water } \\
\text { with } \\
0.1 \% \text { FA }\end{array}$ & Adenosine & Thymidine \\
\hline 50 & 50 & 3.23 & 2.41 \\
\hline 60 & 40 & 3.34 & 2.42 \\
\hline 70 & 30 & 3.92 & 2.56 \\
\hline 80 & 20 & 5.04 & 2.74 \\
\hline 85 & 15 & 6.22 & 2.89 \\
\hline 90 & 10 & 8.60 & 3.06 \\
\hline 95 & 05 & - & - \\
\hline
\end{tabular}




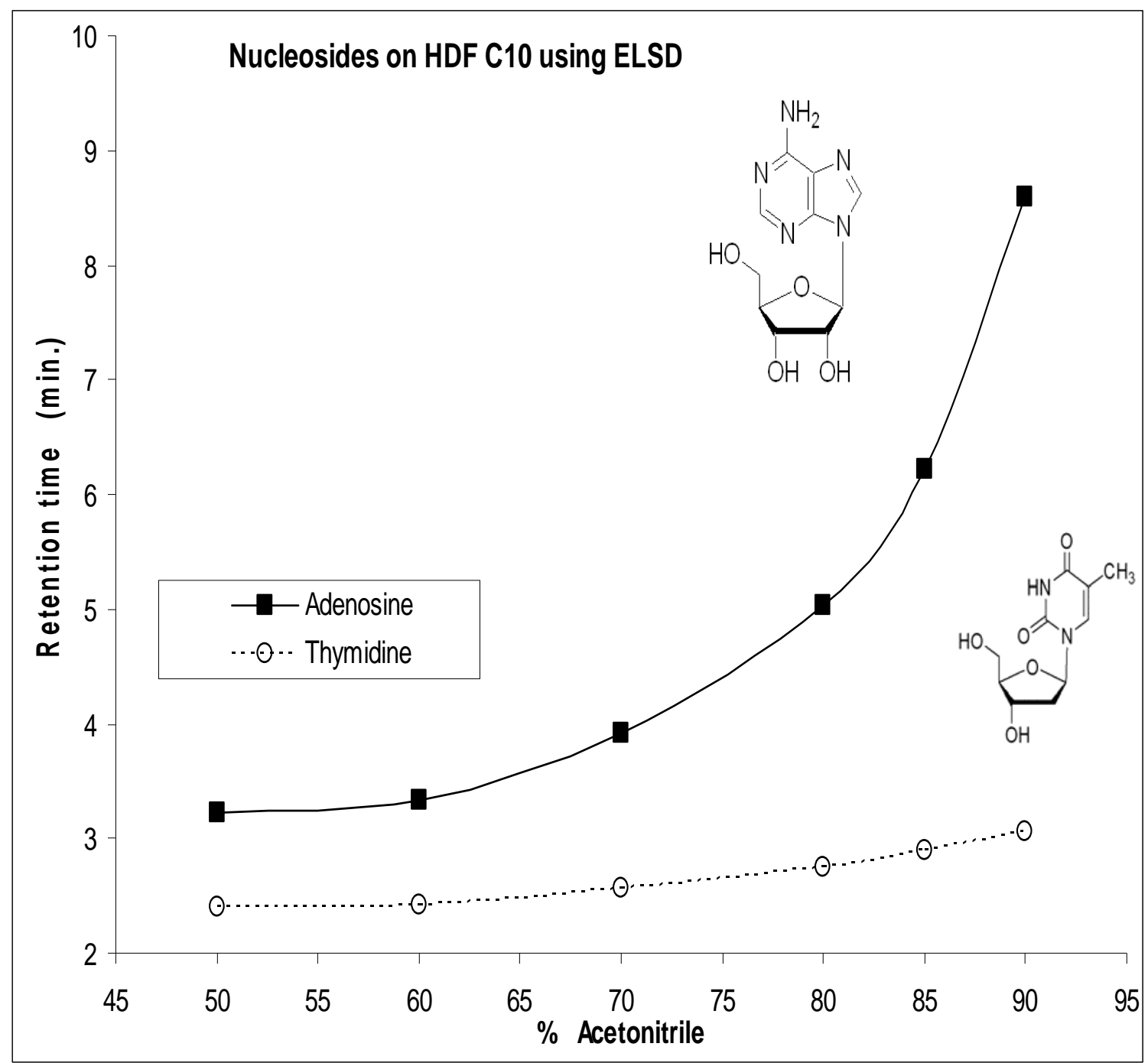

Figure 28. Retention maps of nucleosides on the HDF C10 column using ELSD.

\subsection{Ergogenic Acids}

Table 12 and Table 13 show retention times of creatine and creatinine ergogenic acids respectively with the increasing concentration of acetonitrile solvent from $50 \%$ to 90\%. Figure 29 and Figure 30 are the characteristic ANP retention maps of creatine and 
creatinine respectively. Both analytes display typical ANP retention on the two columns. Creatinine is a cyclic form of creatine which is mainly found in skeletal muscles. As creatine has an open structure with its hydrophilic groups exposed, it exhibits more polarity and shows stronger ANP retention on both columns when compared with the retention of cyclic creatinine.

Table 12. Retention times of ergogenic metabolites on the TDF C8 column using ELSD

\begin{tabular}{|c|c|c|c|}
\hline \multicolumn{2}{|c|}{$\begin{array}{c}\text { Concentration } \\
(\%)\end{array}$} & \multicolumn{2}{|c|}{$\begin{array}{c}\text { Retention time } \\
\text { (min) }\end{array}$} \\
\hline $\begin{array}{c}\text { Acetonitrile } \\
\text { with } \\
0.1 \% \text { FA }\end{array}$ & $\begin{array}{c}\text { DI Water } \\
\text { with } \\
0.1 \% \text { FA }\end{array}$ & Creatine hydrate & Creatinine \\
\hline 50 & 50 & 3.16 & 3.22 \\
\hline 60 & 40 & 3.86 & 3.87 \\
\hline 70 & 30 & 5.22 & 5.14 \\
\hline 75 & 25 & 6.35 & 6.39 \\
\hline 80 & 20 & 8.32 & 8.35 \\
\hline 85 & 15 & 15.02 & 12.90 \\
\hline 90 & 10 & 26.97 & 24.65 \\
\hline
\end{tabular}




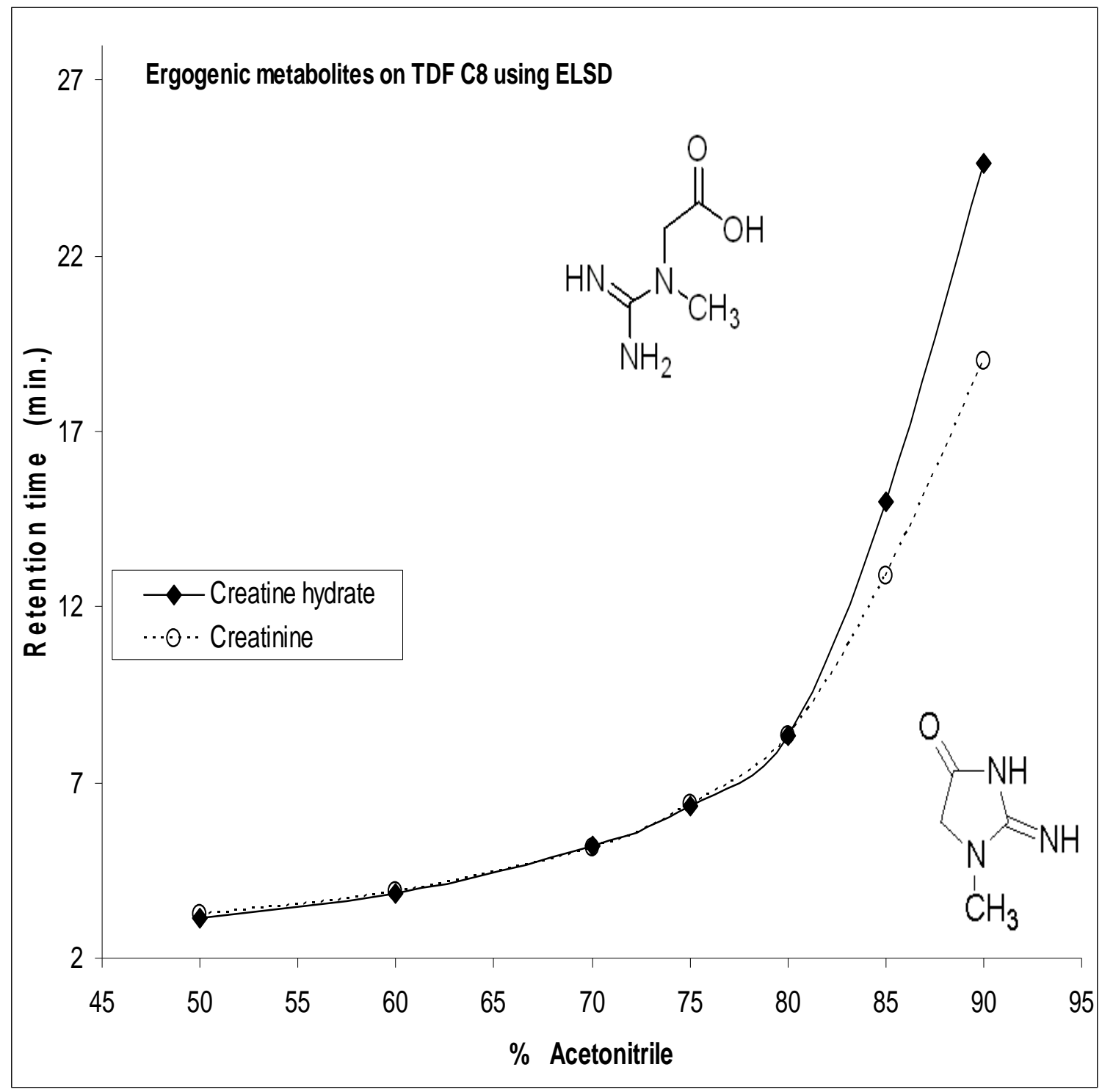

Figure 29. Retention maps of ergogenic metabolites on the TDF C8 column using ELSD. 
Table 13. Retention times of ergogenic metabolites on the HDF C10 column using ELSD

\begin{tabular}{|c|c|c|c|}
\hline \multicolumn{2}{|c|}{$\begin{array}{c}\text { Concentration } \\
\text { (\%) }\end{array}$} & \multicolumn{2}{|c|}{$\begin{array}{l}\text { Retention time } \\
\text { (min) }\end{array}$} \\
\hline $\begin{array}{c}\text { Acetonitrile } \\
\text { with } \\
0.1 \% \text { FA }\end{array}$ & $\begin{array}{l}\text { DI Water } \\
\text { with } \\
0.1 \% \text { FA }\end{array}$ & Creatine hydrate & Creatinine \\
\hline 50 & 50 & 3.12 & 3.09 \\
\hline 60 & 40 & 3.67 & 3.52 \\
\hline 70 & 30 & 4.87 & 4.43 \\
\hline 75 & 25 & 6.16 & 5.36 \\
\hline 80 & 20 & 8.95 & 7.03 \\
\hline 85 & 15 & 13.93 & 10.57 \\
\hline 90 & 10 & - & 19.02 \\
\hline
\end{tabular}

Furthermore, Figure 31 compares the retention of creatinine on the TDF C 8 and HDF C10 columns. Creatinine displays comparatively higher retention on the TDF C8 which has somewhat more of the fluorine moiety influence for retention mechanism than that of HDF C10 column. Hence, it can be postulated that may be the molecules like creatinine show ANP retention due to the polarity of fluorinated moiety. 


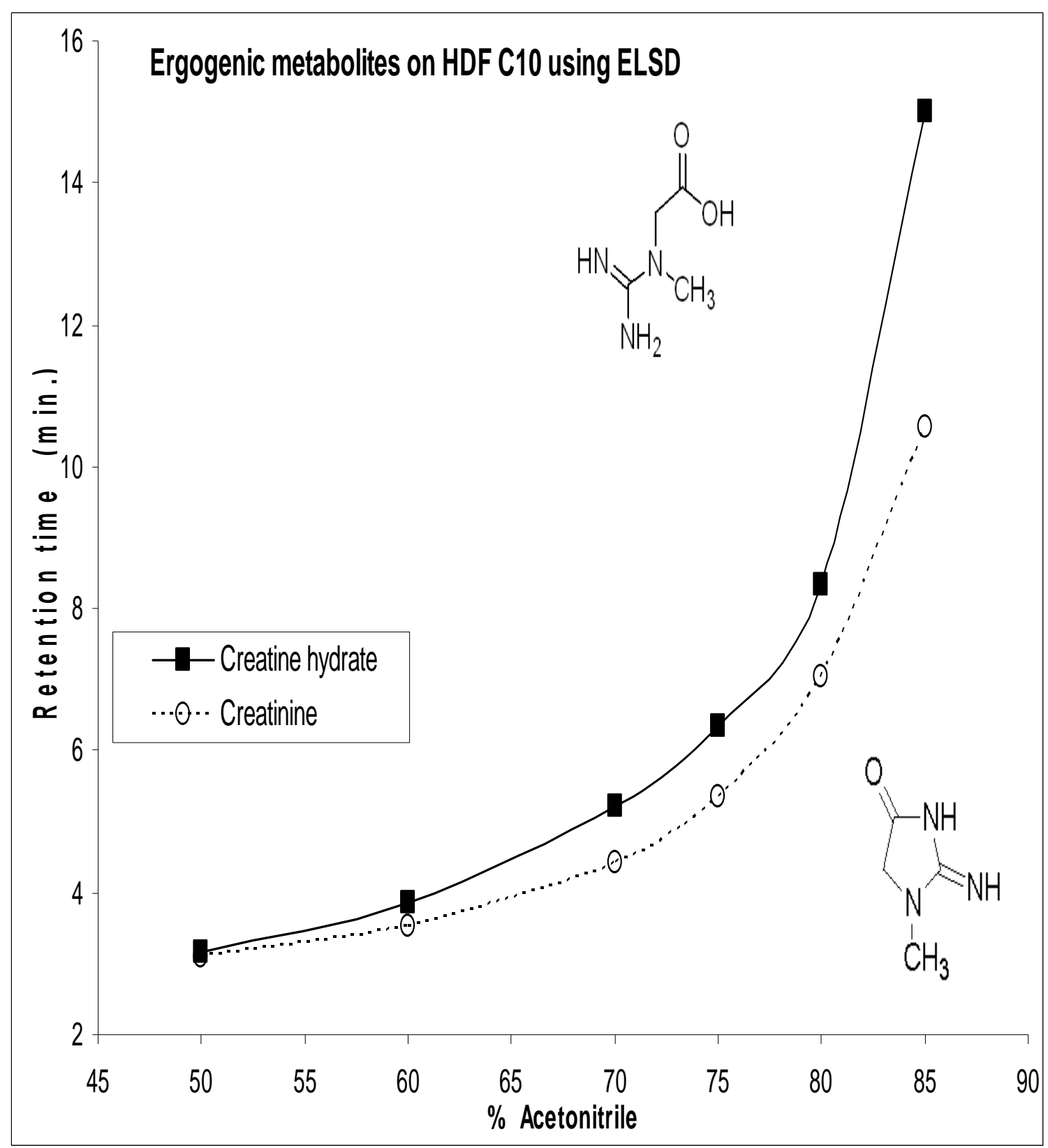

Figure 30. Retention maps of ergogenic metabolites on the HDF C10 column using ELSD. 


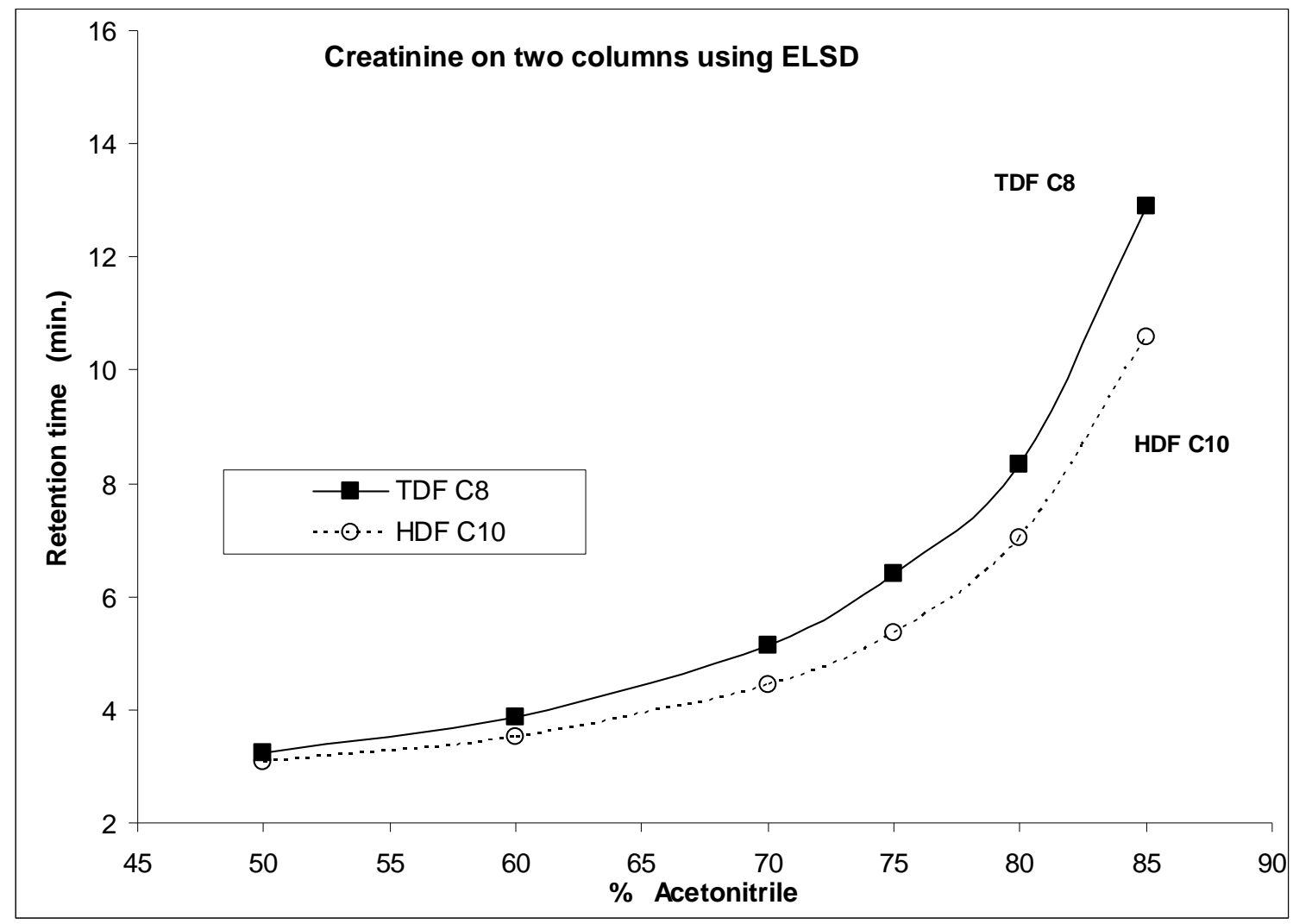

Figure 31. Comparison of retention maps of creatinine on the TDF C8 and HDF C10 columns.

\subsection{Organic Acids}

The small polar fumaric acid and citric acid were tested in order to check their ANP retention using $0.1 \% \mathrm{FA}$ on the HDF $\mathrm{C} 10$ and TDF C8 columns respectively as shown in Table 14. For organic acids, the addition of $0.1 \%$ FA did not result in significant ANP retention although the solutes are highly polar compounds. However, their minute but measurable retention should not be overlooked and can be attributed due to the ANP 
behavior of the columns as shown in Figure 32.

Besides these two organic acids, maleic acid was also analyzed using $0.1 \%$ FA in the similar fashion, but it did not retain at all on both columns eluting at the void volume for all the increasing acetonitrile concentrations. The insignificant or zero ANP retention of these organic acids in the presence of $0.1 \% \mathrm{FA}$ is likely due to the fact that the additive formic acid protonates the organic acids and lowers its ionic character for possible ANP retention. Subsequently, ammonium formate was added to form anions of the organic acids and checked for their possible ANP retention using UV detection. Hence, it is important to choose the proper additive when formulating the ANP binary mobile phase system.

Table 14. Retention times of organic acids for 0.1\%FA additive using ELSD

\begin{tabular}{|c|c|c|c|}
\hline \multicolumn{2}{|c|}{$\begin{array}{c}\text { Concentration } \\
(\%)\end{array}$} & \multicolumn{2}{c|}{$\begin{array}{c}\text { Retention time } \\
\text { (min) }\end{array}$} \\
\hline Acetonitrile & DI Water & Fumaric acid & Citric acid \\
$(0.1 \%$ & $(0.1 \%$ & $\begin{array}{c}\text { (HDF C10 } \\
\text { column) } \\
\text { Formic acid) }\end{array}$ & $\begin{array}{c}\text { Folumn }) \\
\text { Formic acid) }\end{array}$ \\
\hline 50 & 50 & 2.34 & 2.14 \\
\hline 60 & 40 & 2.39 & 2.20 \\
\hline 70 & 30 & 2.42 & 2.33 \\
\hline 80 & 20 & 2.48 & 2.41 \\
\hline 85 & 15 & 2.58 & 2.47 \\
\hline 90 & 10 & 2.59 & 2.59 \\
\hline
\end{tabular}




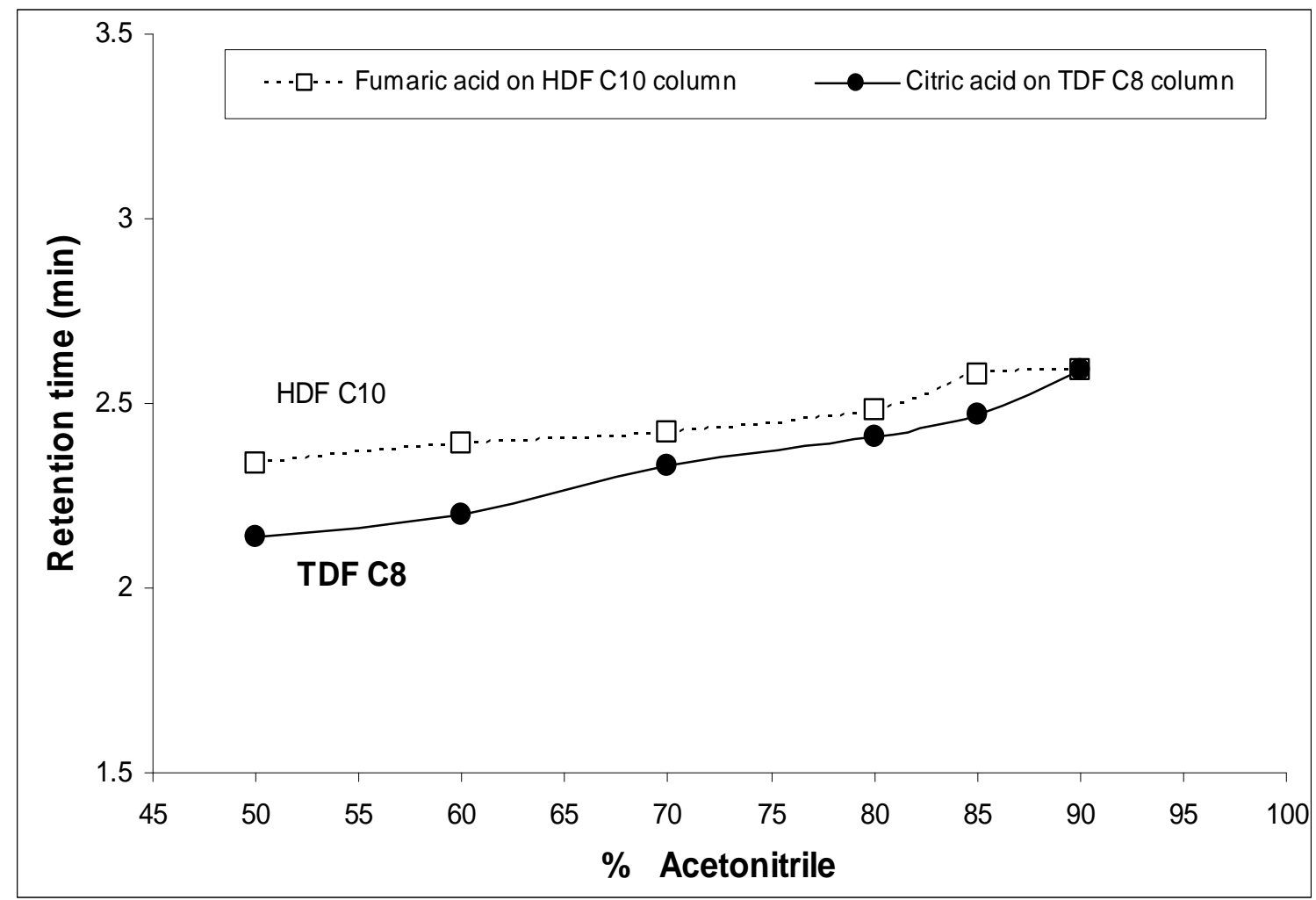

Figure 32. ANP retention of fumaric acid and citric acid with $0.1 \%$ FA additive on HDF C10 column and TDF C8 column respectively using ELSD.

\section{Retention Studies Using UV Detection}

Due to the unavailability of the ELSD, further ANP retention evaluation of the TDF C8 and HDF C10 columns was performed using a UV detector. Different classes of compounds e.g., nucleotide, organic acids and carbohydrates were analyzed to establish their characteristic ANP retention on the fluorinated columns. When fumaric acid, citric acid and maleic acid were analyzed using the ELSD, they were not retained to any extent using $0.1 \% \mathrm{FA}$ as an additive. Therefore, another goal using UV detection was to test 
different types and concentration of additives to achieve higher ANP retention of these solutes.

The use of a higher $\mathrm{pH}$ buffer would deprotonate the organic acids and provide anions for possible ANP retention as the acetonitrile concentration increases. With this consideration, various solvent formulations were made to achieve an appropriate organic acid sample solution and corresponding mobile phase solution with a pH around 6 and not more than 7 using 10 to $15 \mathrm{mM}$ ammonium formate. Whenever the ammonium formate was used, the sample solution and the mobile phase solution were filtered using $0.45 \mu \mathrm{m}$ nylon membrane filter. After checking different concentrations, $15 \mathrm{mM}$ ammonium formate was optimized to make the water mobile phase with a $\mathrm{pH} \sim 6$. For making a $\mathrm{pH} \sim 6$ acetonitrile mobile phase, it was necessary to use $10 \%$ water for dissolving the ammonium formate first and then adding the remainder of the acetonitrile solvent. The dissolution of ammonium formate in lower than $10 \%$ water content recrystalizes it when making the $\mathrm{pH} 6$ acetonitrile mobile phase. Hence, $15 \mathrm{mM}$ ammonium formate was always dissolved in $10 \%$ milliQ water and $90 \%$ acetonitrile to get a $\mathrm{pH} \sim 6$ acetonitrile mobile phase. This water and acetonitrile binary mobile phase system with a $\mathrm{pH} \sim 6$ was used in the following sections for the ANP retention studies of acidic polar molecules.

The organic acids analyzed using UV detection were maleic acid, fumaric acid and succinic acid. They were analyzed using a wavelength of $220 \mathrm{~nm}$ for detection and $0.5 \mathrm{~mL} / \mathrm{min}$ mobile phase flow rate. A 1:40 dilution of the $1 \mathrm{mg} / \mathrm{mL}$ solution made in 
50:50 acetonitrile:water at $\mathrm{pH} \sim 6$ mobile phase condition was used as a sample solution. This sample solution was manually injected to evaluate both the TDF C8 and HDF C10 columns using 50:50, 60:40, 70:30, 80:20, 85:15, 90:10, and 95:05 acetonitrile:water at $\mathrm{pH} \sim 6$ mobile phase compositions. However, surprisingly all three organic acids did not show retention more than 2.5 minutes. As they were injected manually, the run time was kept between 5-8 minutes only, which might be one of the possible reasons for not being able to observe the real sample peak that might have retained longer and have eluted a lot later on. The second possibility is that only the solvent front peaks might have been noted in each case and the HPLC run was stopped for the next injection before the real sample peak appears. The third possibility of not being able to locate the sample peak is that the organic acids might not be absorbing at $220 \mathrm{~nm}$. The fourth possibility is that the absorbance of the additive ammonium formate might be masking the organic acid's sample peaks. So, to overcome the first possibility, all three organic acids were analyzed a second time with 10 minutes or longer run time on both columns. For this set of conditions as well, the same solvent front peak was apparent with the retention time not higher than 2.50 minutes even for 95:05 acetonitrile:water at $\mathrm{pH} \sim 6$ composition. Therefore the conclusion was made that they either were not retained on these columns using the stated experimental conditions or may not be visible using UV detection.

The availability of an MS detection system has provided another choice to check for the retention behavior of the organic acids as in this case the detection is solely based on the molecular weight recognition as determined by the mass/charge ratio. Hence, the 
HPLC-MS analyses of organic acids, subsequently performed, are discussed in the MS detection part of the thesis.

\section{$2.1 \quad$ Nucleotide}

ATP, a nucleotide, displayed good ANP retention on both the TDF C8 and HDF C10

columns. However, the optimized conditions were obtained after many trials as follows. First, $1 \mathrm{mg} / \mathrm{mL}$ sample solution of adenosine-5 ${ }^{`}$ - triphosphate (ATP) was made in 50:50 acetonitrile:water $\mathrm{pH} \sim 6$ solution. Samples at 1:10, 1:20, 1:30, and 1:40 dilution were tested in order to obtain a good chromatographic peak for the 50:50 acetonitrile:water $\mathrm{pH} \sim 6$ mobile phase condition. The 1:20 dilution of a $1 \mathrm{mg} / \mathrm{mL}$ sample solution was optimized and used for the retention studies. A UV wavelength of $254 \mathrm{~nm}$ was selected to monitor the peaks. The mobile phase flow rate was set to $0.5 \mathrm{~mL} / \mathrm{min}$. When this sample solution was run on the TDF C8 and HDF C10 columns with increasing acetonitrile:water from 50:50 to 95:05 compositions, it had a retention time no longer than 2.54 minutes. Later on it was realized that the run time of each injection was not sufficient enough to track the real sample peak that had been retained much longer. So as described earlier in the case of organic acids, the retention times of solvent front peak were monitored. Therefore, further analyses were carried out having a run time of at least 15 minutes or longer in order to check for highly retained peaks when the acetonitrile concentration increases.

In the second trial, a 1:20 dilution of the $1 \mathrm{mg} / \mathrm{mL}$ ATP sample solution made in 50:50 acetonitrile:water at $\mathrm{pH} \sim 6$, was analyzed for 50:50, 60:40, 70:30, and 80:20 mobile phase compositions on both columns with run time up to 15 minutes. The resultant 
peaks were confusing, as the sample peak was being obscured by the solvent front in each trial. It has also been observed that as the concentration of acetonitrile increases, the intensity of the solvent front peak increased 2-3 fold in comparison with the intensity of the retained sample peak.

Finally, a 1:20 dilution of the 1mg/mL ATP dissolved in 80:20 acetonitrile:water at $\mathrm{pH} \sim 6$ sample solution provided very symmetric peaks. Whenever the sample peaks displayed shoulders or split, they were confirmed by analyzing the sample for that mobile phase condition with a different UV wavelength of $220 \mathrm{~nm}$ instead of $254 \mathrm{~nm}$. For $220 \mathrm{~nm}$, the absence of ATP absorption was exhibited as a negative peak which was the sample peak and it continues to retain as the acetonitrile concentration increases. Accordingly the confirmed positive sample peaks were studied for ATP using a wavelength of $254 \mathrm{~nm}$ on both the TDF C8 and HDF C10 columns.

Table 15 gives the retention times of ATP as the concentration of acetonitrile increases. ATP displayed very high ANP retention up to approximately 42 minutes for $90 \%$ acetonitrile on the HDF C10 column. The right hand side part of Table 15 represents the analysis of ATP on the HDF C10 column using 1:5 dilution of a $1 \mathrm{mg} / \mathrm{mL}$ sample solution. In this case, a very sharp ANP retention was observed for the short range of $70 \%$ to $88 \%$ acetonitrile, and is plotted in Figure 33 .

The left hand side part of Table 15 represents the analysis of ATP using a 1:20 dilution of the $1 \mathrm{mg} / \mathrm{mL}$ sample solution on both columns. Figure 34 shows the characteristic retention maps of ATP on both columns as the amount of acetonitrile 
increases. The aqueous normal phase retention maps of ATP analyzed on TDF C8 and HDF C10 are completely overlapping. This fact confirms the comparable contribution of the fluorinated moiety and the silica hydride layer in the aqueous normal phase retention mechanism of ATP.

Table 15. Retention times of ATP on both TDF C8 and HDF C10 columns using UV detection

\begin{tabular}{|c|c|c|c|c|c|c|}
\hline \multicolumn{2}{|c|}{$\begin{array}{c}\text { Concentration } \\
(\%)\end{array}$} & \multicolumn{2}{|c|}{$\begin{array}{l}\text { Retention time } \\
\text { (min.) }\end{array}$} & \multicolumn{2}{|c|}{$\begin{array}{c}\text { Concentration } \\
\%\end{array}$} & \multirow{2}{*}{$\begin{array}{c}\begin{array}{c}\text { Retention } \\
\text { time } \\
\text { (min) }\end{array} \\
\text { HDF } \\
\text { C10 } \\
\text { (200 ppm } \\
\text { ATP } \\
\text { solution) }\end{array}$} \\
\hline $\begin{array}{c}\text { Acetonitrile } \\
+ \\
(15 \mathrm{mM} \\
\text { Ammonium- } \\
\text { formate })\end{array}$ & $\begin{array}{c}\text { DI Water } \\
+ \\
(15 \mathrm{mM} \\
\text { Ammonim- } \\
\text { formate })\end{array}$ & $\begin{array}{c}\text { TDF C8 } \\
\\
\text { (50 ppm } \\
\text { ATP } \\
\text { solution) }\end{array}$ & $\begin{array}{c}\text { HDF } \\
\text { C10 } \\
\text { (50 ppm } \\
\text { ATP } \\
\text { solution) }\end{array}$ & $\begin{array}{c}\text { Acetonitrile } \\
+ \\
(15 \mathrm{mM} \\
\text { Ammonium - } \\
\text { formate })\end{array}$ & $\begin{array}{c}\text { DI water } \\
+ \\
(15 \mathrm{mM} \\
\text { Ammonium } \\
\text { formate })\end{array}$ & \\
\hline 50 & 50 & 1.69 & 1.67 & 70 & 30 & 2.11 \\
\hline 60 & 40 & 1.76 & 1.78 & 75 & 25 & 2.39 \\
\hline 70 & 30 & 1.96 & 2.03 & 80 & 20 & 3.65 \\
\hline 75 & 25 & 2.40 & 2.45 & 82 & 18 & 4.78 \\
\hline 80 & 20 & 3.25 & 3.19 & 84 & 16 & 6.86 \\
\hline 83 & 17 & 5.75 & 6.38 & 86 & 14 & 11.42 \\
\hline 85 & 15 & 8.81 & 9.61 & 88 & 12 & 23.44 \\
\hline 87 & 13 & 15.51 & 16.55 & - & - & - \\
\hline 90 & 10 & 38.91 & 42.40 & - & - & - \\
\hline
\end{tabular}




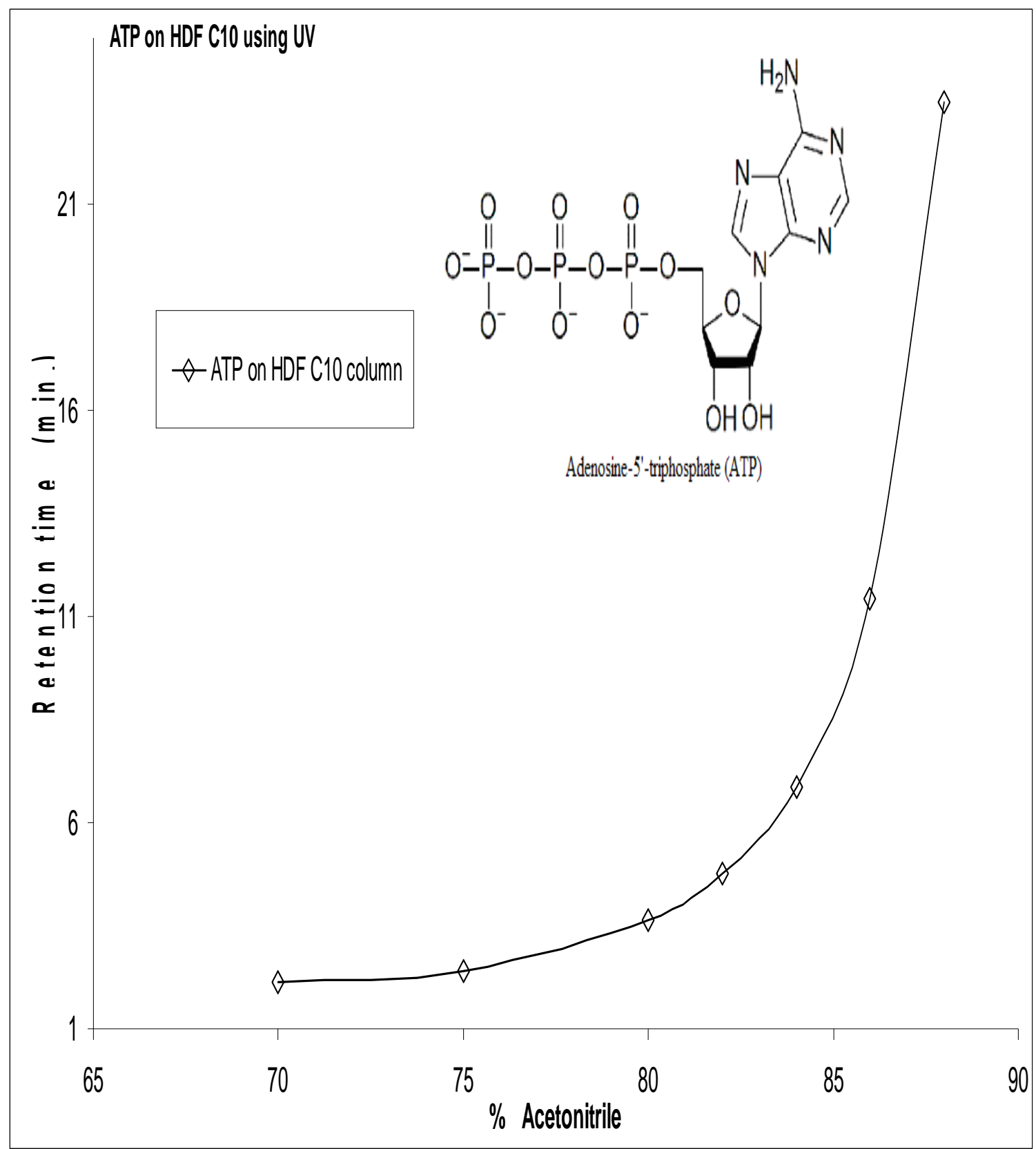

Figure 33. ANP retention of ATP on the HDF C10 column using UV detection. 


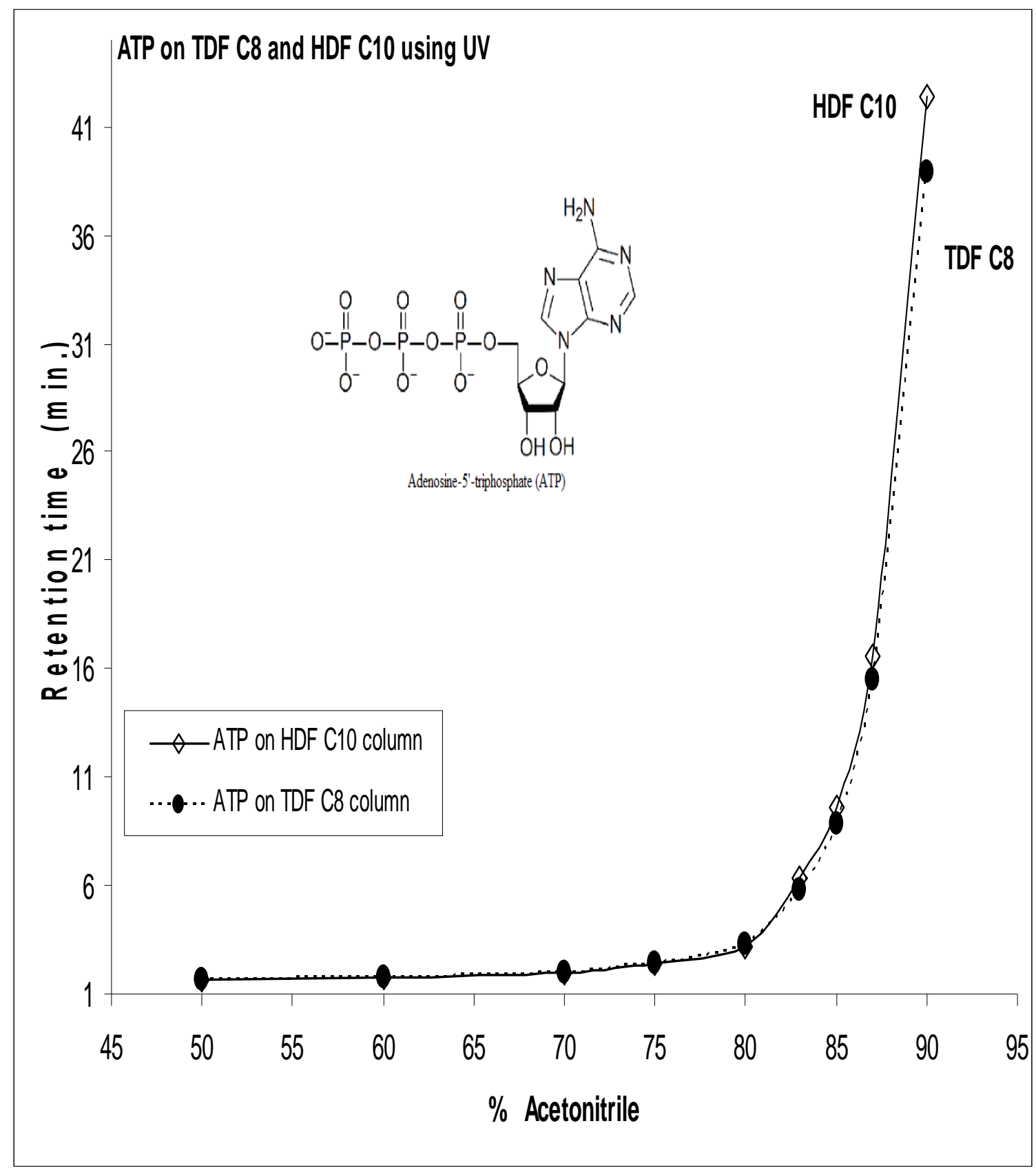

Figure 34. Comparison of ANP retention of ATP on the HDF C10 and TDF C8 columns using UV detection. 


\subsection{Carbohydrates}

Carbohydrates were another class of compounds analyzed to check their possible ANP retention on the fluorinated columns. Specially, the monosaccharides glucose and fructose were run to optimize the experimental conditions using UV detection. First, they were run using a $0.1 \% \mathrm{FA}$ additive and afterwards $15 \mathrm{mM}$ ammonium formate buffered ( $\mathrm{pH} 6$ ) mobile phase conditions.

As shown in Table 16, under the conditions of $\mathrm{pH} \sim 6$, glucose and fructose both have ANP retention as the concentration of acetonitrile increases on the TDF C8 column. A similar trend of ANP retention was obtained on the HDF C10 column as well. For analyzing glucose and fructose, a $220 \mathrm{~nm}$ UV wavelength was used. Since carbohydrates do not carry UV active chromophores, the lack of absorption as a negative peak was monitored as a chromatogram where the background mobile phase was absorbing in this region. The monitoring of the negative sample peaks showed some ANP retention in the case of $0.1 \% \mathrm{FA}$ and very high ANP retention when the $\mathrm{pH} \sim 6$ mobile phase was used on both columns. The mobile phase flow rate was set to $0.5 \mathrm{~mL} / \mathrm{min}$ and $20 \mu \mathrm{L}$ of the sample was injected for each run in this analysis. For the studies with the $0.1 \% \mathrm{FA}$ additive, glucose and fructose were dissolved to make a $1 \mathrm{mg} / \mathrm{mL}$ sample solution in 50:50 acetonitrile:water premixed with $0.1 \% \mathrm{FA}$. In the case of the $\mathrm{pH} \sim 6$ conditions, glucose and fructose were dissolved to make a $1 \mathrm{mg} / \mathrm{mL}$ sample solution using 50:50 acetonitrile:water $\mathrm{pH} \sim 6$ mobile phase.

Table 16 presents the retention times for ANP retention on the TDF C8 column using $0.1 \% \mathrm{FA}$ and $15 \mathrm{mM}$ ammonium formate additives and Figure 35 illustrates the 
increasing ANP retention of glucose and fructose when $15 \mathrm{mM}$ ammonium formate was used instead of $0.1 \% \mathrm{FA}$.

Table 16. Retention times of glucose and fructose on the TDF C8 column with $0.1 \%$ FA and 15mM ammonium formate additives using UV detection

\begin{tabular}{|c|c|c|c|c|c|c|c|}
\hline \multicolumn{2}{|c|}{$\begin{array}{c}\text { Concentration } \\
\text { (\%) }\end{array}$} & \multicolumn{2}{|c|}{$\begin{array}{l}\text { Retention time } \\
\text { (min) }\end{array}$} & \multicolumn{2}{|c|}{$\begin{array}{c}\text { Concentration } \\
\text { (\%) }\end{array}$} & \multicolumn{2}{|c|}{$\begin{array}{l}\text { Retention time } \\
\text { (min) }\end{array}$} \\
\hline $\begin{array}{c}\text { Acetonitrile } \\
(0.1 \% \mathrm{FA})\end{array}$ & $\begin{array}{c}\text { DI } \\
\text { Water } \\
(0.1 \% \\
\text { FA) }\end{array}$ & Glucose & Fructose & $\begin{array}{l}\text { Acetonitrile } \\
\text { (15mM } \\
\text { Ammonium } \\
\text { formate) }\end{array}$ & $\begin{array}{l}\text { DI Water } \\
\text { (15mM } \\
\text { ammonium } \\
\text { formate })\end{array}$ & Glucose & Fructose \\
\hline 50 & 50 & 2.21 & 2.21 & 50 & 50 & 2.33 & 2.25 \\
\hline 60 & 40 & 2.23 & 2.13 & 60 & 40 & 2.34 & 2.38 \\
\hline 70 & 30 & 2.18 & 2.16 & 70 & 30 & 2.48 & 2.52 \\
\hline 80 & 20 & 2.50 & 2.52 & 80 & 20 & 2.97 & 3.02 \\
\hline 85 & 15 & 2.58 & 2.57 & 85 & 15 & 3.54 & 3.58 \\
\hline 90 & 10 & 2.72 & 2.68 & 90 & 10 & 4.59 & 4.62 \\
\hline 95 & 05 & 2.85 & 2.81 & 95 & 05 & 6.88 & 6.96 \\
\hline
\end{tabular}




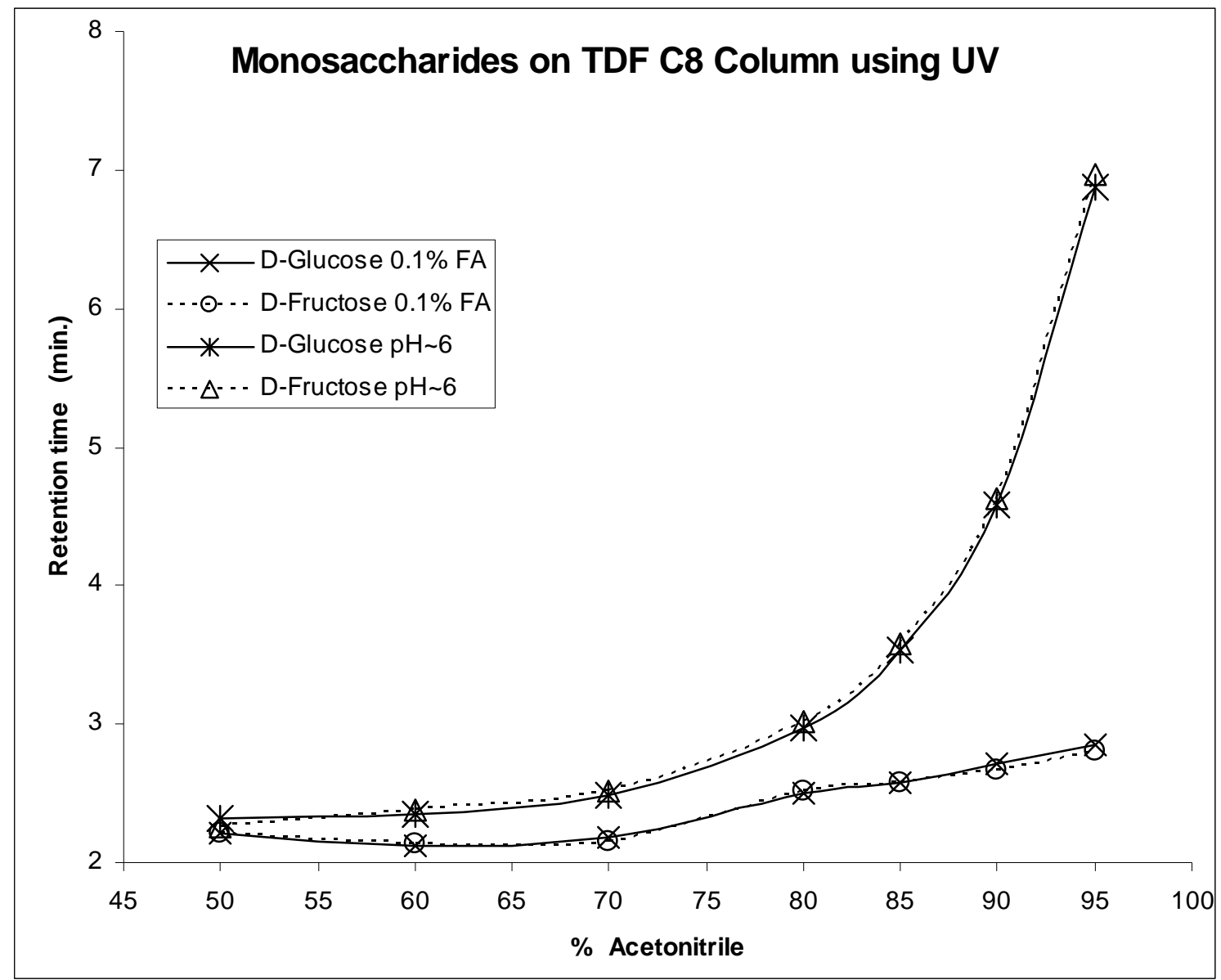

Figure 35. Retention maps of glucose and fructose with $0.1 \%$ FA and $15 \mathrm{mM}$ ammonium formate additives using UV detection.

Table 17 lists the retention times and Figure 36 demonstrates the corresponding characteristic retention maps of glucose and fructose observed on the TDF C8 and HDF C10 columns using the $15 \mathrm{mM}$ ammonium formate mobile phase conditions. As can be seen, both monosaccharides retain slightly more on the HDF C10 column which might be due to more of the underlying silica hydride contribution to the ANP retention mechanism of glucose and fructose than the attached fluorinated moiety. 
Table 17. Retention times of glucose and fructose on both the TDF C8 and HDF C10 columns with $15 \mathrm{mM}$ ammonium formate additive using UV detection

\begin{tabular}{|c|c|c|c|c|c|}
\hline \multirow{2}{*}{\multicolumn{2}{|c|}{$\begin{array}{c}\text { Concentration } \\
(\%)\end{array}$}} & \multicolumn{4}{|c|}{$\begin{array}{l}\text { Retention time } \\
\text { (min) }\end{array}$} \\
\hline & & \multicolumn{2}{|c|}{ TDF C8 } & \multicolumn{2}{|c|}{ HDF C10 } \\
\hline $\begin{array}{l}\text { Acetonitrile } \\
\text { (15mM } \\
\text { Ammonium } \\
\text { formate })\end{array}$ & $\begin{array}{c}\text { DI Water } \\
\text { (15mM } \\
\text { Ammonium } \\
\text { formate) }\end{array}$ & Glucose & Fructose & Glucose & Fructose \\
\hline 50 & 50 & 2.33 & 2.25 & 2.22 & 2.19 \\
\hline 60 & 40 & 2.34 & 2.38 & 2.35 & 2.36 \\
\hline 70 & 30 & 2.48 & 2.52 & 2.61 & 2.60 \\
\hline 80 & 20 & 2.97 & 3.02 & 3.24 & 3.25 \\
\hline 90 & 10 & 4.59 & 4.62 & 5.37 & 5.36 \\
\hline
\end{tabular}




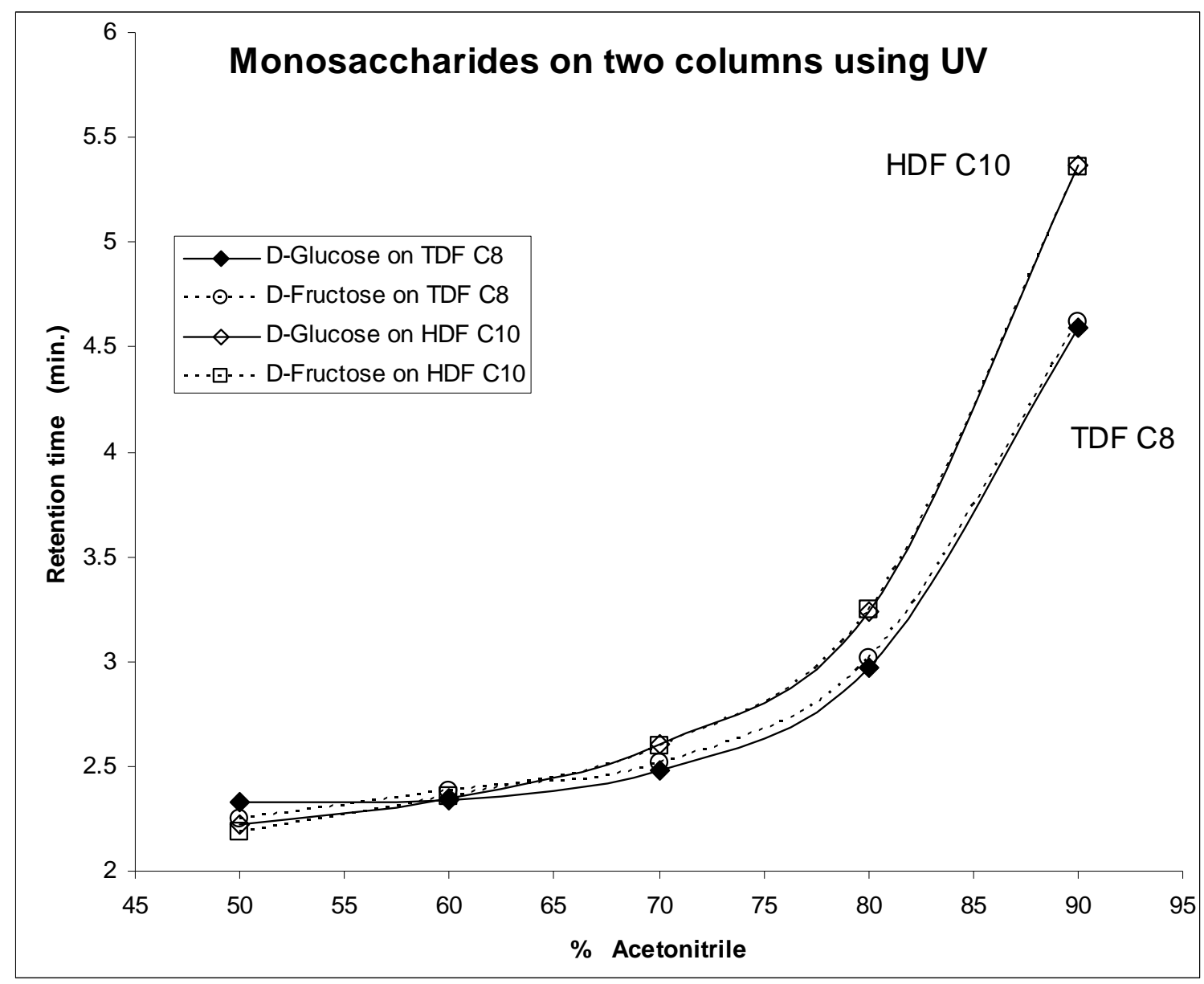

Figure 36. Comparison of retention maps of glucose and fructose on the TDF C8 and HDF C10 columns using UV detection.

The disaccharides turanose and trehalose were also analyzed on the TDF C8 column using UV detection. The flow rate was set to $0.5 \mathrm{~mL} / \mathrm{min}$, and a UV absorption wavelength of $220 \mathrm{~nm}$ was used. A sample solution at a concentration of $3 \mathrm{mg} / \mathrm{mL}$ was made using 50:50 acetonitrile:water $\mathrm{pH} \sim 6$ mobile phase composition. The injection volume was $20 \mu \mathrm{L}$. The negative peaks were measured to record the ANP retention times as shown in Table 18. Both turanose and trehalose displayed good ANP retention 
on the TDF C8 column and the same trend would occur on the HDF C10 column as well. The retention maps using the UV detection are plotted along with the retention maps obtained using MS detection in a subsequent section of the thesis. The ANP retention data of turanose and trehalose obtained using UV detection were validated by comparing them against the ANP retention data obtained by MS detection discussed in the later section.

Table 18. Retention times of disaccharides on the TDF C8 column using UV detection

\begin{tabular}{|c|c|c|c|}
\hline \multicolumn{2}{|c|}{$\begin{array}{c}\text { Concentration } \\
(\%)\end{array}$} & \multicolumn{2}{|c|}{$\begin{array}{c}\text { Retention time } \\
\text { (min) }\end{array}$} \\
\hline & & \multicolumn{2}{|c|}{$\begin{array}{c}\mathbf{U V} \\
(0.5 \mathrm{~mL} / \mathrm{min})\end{array}$} \\
\hline $\begin{array}{l}\text { Ammonium } \\
\text { formate) }\end{array}$ & $\begin{array}{l}\text { Ammonium } \\
\text { formate) }\end{array}$ & Turanose & Trehalose \\
\hline 50 & 50 & 2.23 & 2.21 \\
\hline 60 & 40 & 2.32 & 2.33 \\
\hline 70 & 30 & 2.54 & 2.54 \\
\hline 80 & 20 & 3.15 & 3.15 \\
\hline 90 & 10 & 5.24 & 5.29 \\
\hline
\end{tabular}




\section{Retention Studies Using LC-MS Detection}

The main purpose of using a mass spectrometer was to check the ANP retention of organic acids and carbohydrates in the evaluation of the TDF C8 and HDF C10 columns. Since these solutes showed longer retention under the $\mathrm{pH} \sim 6$ condition of the mobile phase, they were analyzed using $15 \mathrm{mM}$ ammonium formate. In parallel, these solutes were also analyzed to establish ANP retention using acetone as the organic solvent component of the ANP binary mobile phase. Finally, some of the ANP retention data of amino acids and carbohydrates obtained using ELSD and UV detectors were verified using the more sensitive and universal MS detector.

In the mass spectrometer, the retained analytes from the HPLC were ionized using an electrospray ionization (ESI) source and the molecular ions were scanned by a quadrupole mass analyzer for detection. The quadrupole mass analyzer was set to scan over a mass range from $40 \mathrm{amu}$ to $400 \mathrm{amu}$ for all solutes except for cellobiose octaacetate for which scanning was set from $40 \mathrm{amu}$ to $1000 \mathrm{amu}$ as it has higher molecular weight. The Q1 MS scan mode was used for the negative ion polarity scanning and Q3 MS scan mode was used for the positive ion polarity scanning. The data acquisition time interval was set to 10 minutes (it was extended for longer retaining solutes) and the flow rate of the mobile phase was set to $0.4 \mathrm{~mL} / \mathrm{min}$. Lower flow rates of $0.2 \mathrm{~mL} / \mathrm{min}$ and $0.3 \mathrm{~mL} / \mathrm{min}$ gave poor signal to noise ratio with peak broadening, hence $0.4 \mathrm{~mL} / \mathrm{min}$ was the optimized condition. Q1 and Q3 analyzers were set to provide unit resolution for the analysis. 
Table 19. Molecular weights and molecular ions monitored for ANP retention study of (A) organic acids (B) amino acids, and (C) carbohydrates

(A)

\begin{tabular}{|c||c|c|}
\hline $\begin{array}{c}\text { Organic } \\
\text { acids }\end{array}$ & $\begin{array}{c}\text { M.Wt. } \\
\text { g/mol }\end{array}$ & $\begin{array}{c}\text { [M-1] }^{-} \\
\text {focused }\end{array}$ \\
\hline Maleic acid & 116.10 & 115.0 \\
\hline Fumaric acid & 116.07 & 115.0 \\
\hline Succinic acid & 118.09 & 117.1 \\
\hline Citric acid & 192.12 & 190.9 \\
\hline
\end{tabular}

(B)

\begin{tabular}{|c|c|c|}
\hline Amino acids & $\begin{array}{c}\text { M.Wt. } \\
\text { g/mol }\end{array}$ & $\begin{array}{c}\mathbf{M}^{\mathbf{M}+\mathbf{1}} \mathbf{f}^{+} \\
\text {focused }\end{array}$ \\
\hline ARG & 174.20 & 175.70 \\
\hline \hline ASP & 133.10 & 134.10 \\
\hline PHE & 165.19 & 166.80 \\
\hline TYR & 181.19 & 182.60 \\
\hline
\end{tabular}

(C)

\begin{tabular}{|c|c|c|}
\hline Carbohydrates & $\begin{array}{c}\text { M. Wt. } \\
\text { g/mol }\end{array}$ & $\begin{array}{c}\text { [M+Na }^{+} \\
\mathbf{N a}=\mathbf{2 3 g} / \mathbf{m o l}\end{array}$ \\
\hline \hline Fructose & 180.16 & 203.00 \\
\hline Glucose & 180.16 & 203.00 \\
\hline \hline Ribose & 150.13 & 173.13 \\
\hline \hline Sucrose & 342.30 & 365.30 \\
\hline Lactose & 342.30 & 365.30 \\
\hline Maltose & 342.30 & 365.30 \\
\hline Turanose & 342.30 & 365.30 \\
\hline $\begin{array}{c}\text { Trehalose } \\
\text { anhydrous }\end{array}$ & 342.30 & 365.30 \\
\hline $\begin{array}{l}\text { Cellobiose } \\
\text { octaacetate }\end{array}$ & 678.20 & 701.2 \\
\hline
\end{tabular}


Sample solution concentrations of $0.1 \mathrm{mg} / \mathrm{mL}$ to $1 \mathrm{mg} / \mathrm{mL}$ were tried for each class of solutes; however, lower concentrations than $1 \mathrm{mg} / \mathrm{mL}$ with higher injection volumes varying from $5 \mu \mathrm{L}$ to $20 \mu \mathrm{L}$ resulted in peak splitting and lower signal to noise ratio. Hence, a concentration of $1 \mathrm{mg} / \mathrm{mL}$ was optimized for all the samples and the injection volume was varied from $0.5 \mu \mathrm{L}$ to $5 \mu \mathrm{L}$ with the desired value of the threshold being from 50 to 80 to achieve symmetric high efficiency peaks. The acquired data were monitored as total ion current versus time (TIC chromatogram), from which the full mass spectrum displaying different molecular ions was obtained. Once the parent molecular ion or related adduct ion $\mathrm{m} / \mathrm{z}$ (mass to charge) ratio was determined, that mass could be selected to get single ion monitoring versus time (SIM chromatogram) in order to track the ANP retention of that particular solute on both the fluorinated columns. The width of SIM window was set to $0.5 \mathrm{amu}$ with a value of 3 cycles per second (CPS) monitoring frequency for all the results discussed in the following sections. The SIM gave better signal to noise ratio for the selected molecular ion than the TIC.

Table 19 presents the molecular weights and the molecular ions monitored to study ANP and reversed phase (RP) behavior of the TDF C8 and HDF C10 columns using isocratic mobile phase conditions. For the analysis of organic acids, their $[\mathrm{M}-1]^{-1}$ molecular ions were monitored using the Q1 MS negative ion mode; and for the analysis of amino acids, their $[\mathrm{M}+1]^{+1}$ molecular ions were detected using the Q3 MS positive ion mode. In the case of carbohydrates, their sodium adducts as $[\mathrm{M}+\mathrm{Na}]^{+1}$ ions were monitored using the Q3 MS positive ion mode. In the case of amino acids and organic acids, the use of $0.1 \% \mathrm{FA}$ and $15 \mathrm{mM}$ ammonium formate additives respectively 
provided the required protons for the formation of the molecular ions. However, in the case of carbohydrates, the glassware used or the solvent being used were believed to be able to provide sufficient sodium ions to produce sodium adducts of monosaccharide and disaccharide solutes monitored as the $[\mathrm{M}+\mathrm{Na}]^{+1}$ adduct. Secondly, it is necessary to point out that during the MS analysis of carbohydrates and amino acids, the Q3 MS positive polarity mode was off calibrated by 3 to $4 \mathrm{amu}$, and hence the mass spectra of these molecules were obtained as $[\mathrm{M}+1]^{+1}+4$ amu for amino acids and $[\mathrm{M}+\mathrm{Na}]^{+1}+3$ amu for carbohydrates. This off calibration was overcome later on by recalibrating this scan mode. Therefore, for the purpose of the ease of illustration, some of the recorded values of $\mathrm{m} / \mathrm{z}$ mass units describing amino acids' and carbohydrates' molecular ions are edited by subtracting from them 4 and 3 mass units respectively and these values are placed in the tables and in the figures of the following sections.

\subsection{Organic Acids}

The small polar organic acids were analyzed using $15 \mathrm{mM}$ ammonium formate to determine their ANP retention on both the TDF C8 and HDF C10 columns. The sample solution was made as $1 \mathrm{mg} / \mathrm{mL}$ using 50:50 acetonitrile:water with $15 \mathrm{mM}$ ammonium formate to achieve a $\mathrm{pH}$ between 6-7. The flow rate of the mobile phase was kept at $0.4 \mathrm{~mL} / \mathrm{min}$ and the injection volume was set to $1.0 \mu \mathrm{L}$. The threshold value was set from 50 to 80 to achieve sharp and symmetric peaks appropriate to study ANP retention. For the binary mobile phase system, acetonitrile was made by dissolving 15 
$\mathrm{mM}$ ammonium formate in $10 \%$ water which was then brought to the correct volume by adding acetonitrile and water was made by dissolving $15 \mathrm{mM}$ ammonium formate in milliQ water.

First, the total ion chromatogram was recorded for each organic acid using a higher concentration; this sample gives a low intensity broad peak indicating the presence of the solute. From this TIC chromatogram, the mass spectrum was collected to find the $\mathrm{m} / \mathrm{z}$ value of $[\mathrm{M}-1]^{-1}$ molecular ion. Table 19 (A) lists the molecular weight values of the organic acids and the $\mathrm{m} / \mathrm{z}$ values of their $[\mathrm{M}-1]^{-1}$ molecular ions. The Q1 MS negative ion mode was accurately calibrated and hence gave very close values to the expected [M$1]^{-1}$ molecular ions.

Figure 37 shows the mass spectra of maleic acid, fumaric acid, succinic acid, and citric acid with $\mathrm{m} / \mathrm{z}$ values of $115.0 \mathrm{amu}, 115.0 \mathrm{amu}, 117.1 \mathrm{amu}$, and $190.9 \mathrm{amu}$ respectively for their $[\mathrm{M}-1]^{-1}$ molecular ions collected in the ESI negative polarity scan mode. On the basis of these $\mathrm{m} / \mathrm{z}$ values of molecular ions, single ion monitoring (SIM) chromatograms were obtained to study the ANP retention of the organic acids. Figure 38 shows an example of the mass spectroscopic data reporting format for succinic acid at 50:50 acetonitrile:water $\mathrm{pH} \sim 6$ ANP mobile phase condition. The top plot represents the TIC chromatogram, the middle one is the mass spectrum of succinic acid identifying the $117.0 \mathrm{~m} / \mathrm{z}$ value, and the last plot is the SIM chromatographic peak that is used to monitor the solute as the $\%$ of acetonitrile increases in the mobile phase composition. Similarly, a TIC chromatogram, a mass spectrum and a SIM chromatogram for the ANP retention study were obtained for all the solutes analyzed using the MS detector. 


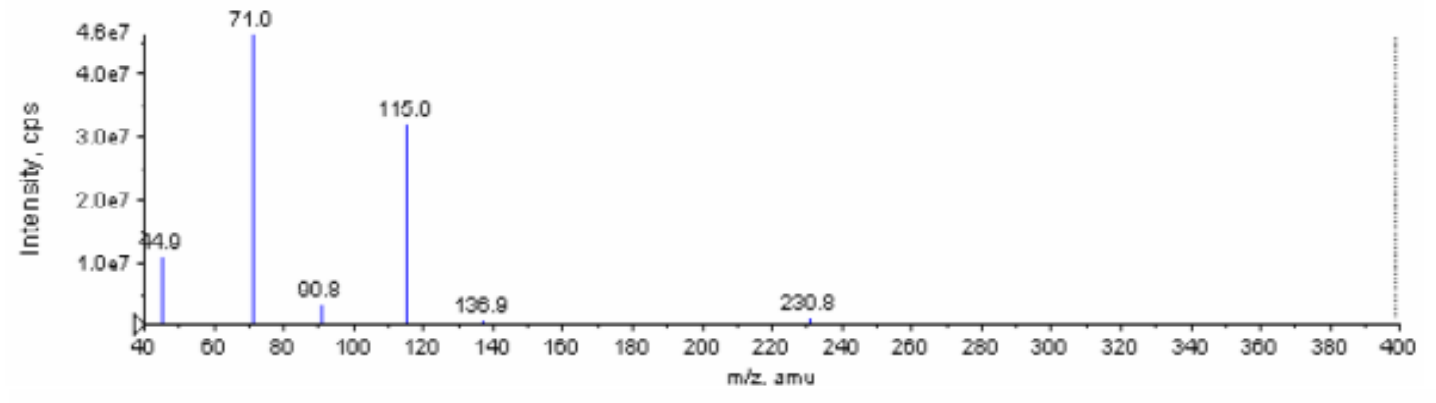

D. Q1:2.304 min from Sample 1 (FUM-1) of \$1FUMneg001TIC5050.wiff

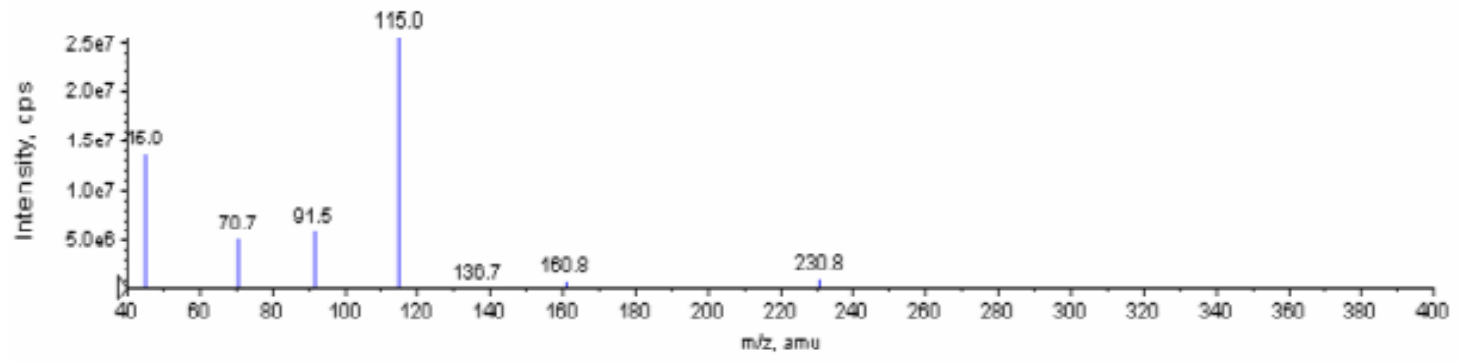

- -Q1:2.505 min from Sample 2 (SUC-2) of \#1SUCneg001TIC5050 miff

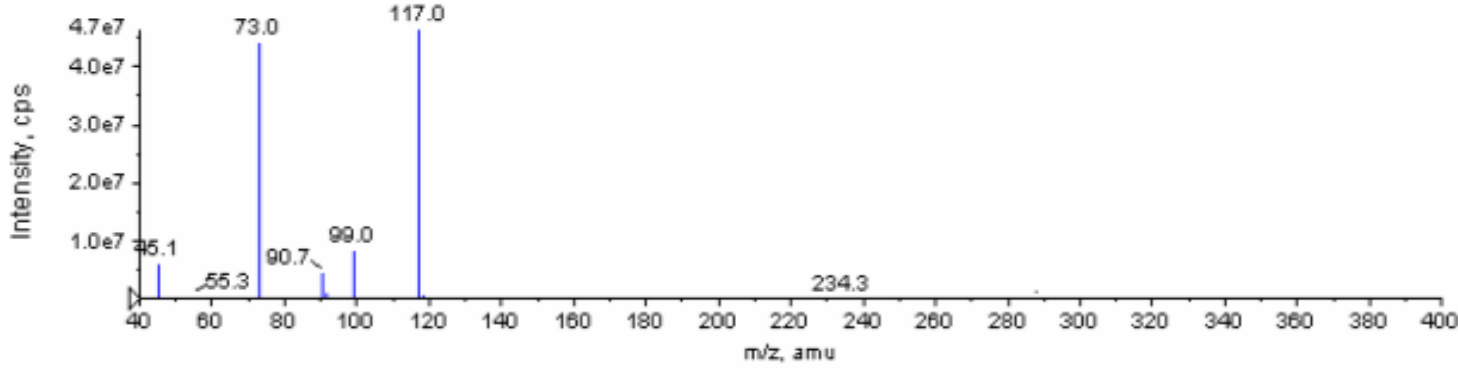

-Q1: 2.454 min from Sample 2 (CIT-2) of \#1CITne g001TIC5050 wiff

$\operatorname{Max} 4.2 \mathrm{e} 7 \mathrm{cps}$

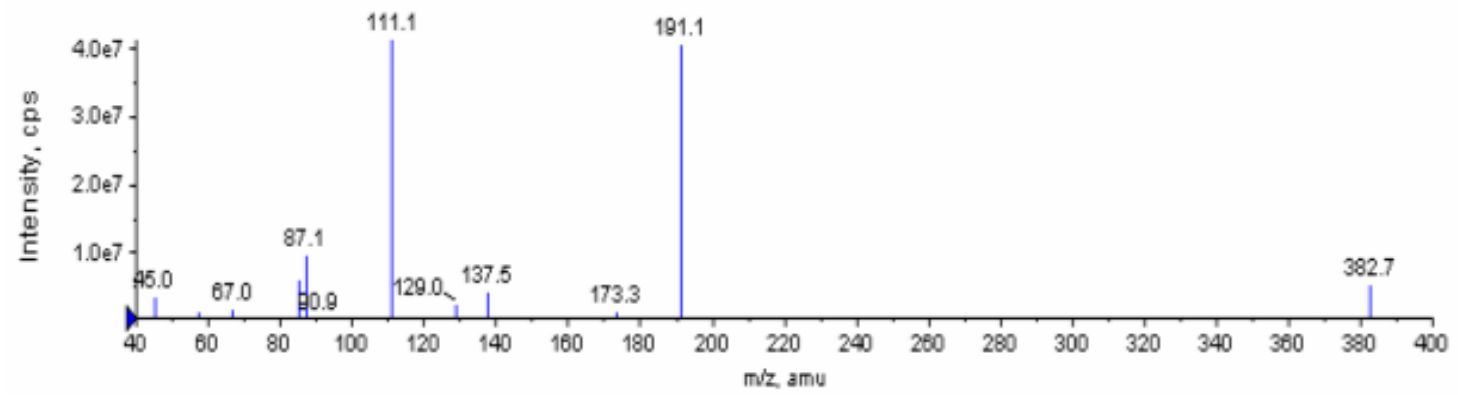

Figure 37. ESI mass spectra of maleic acid, fumaric acid, succinic acid, and citric acid as $[\mathrm{M}-1]^{-1}$ molecular ion recorded using negative polarity mode. 


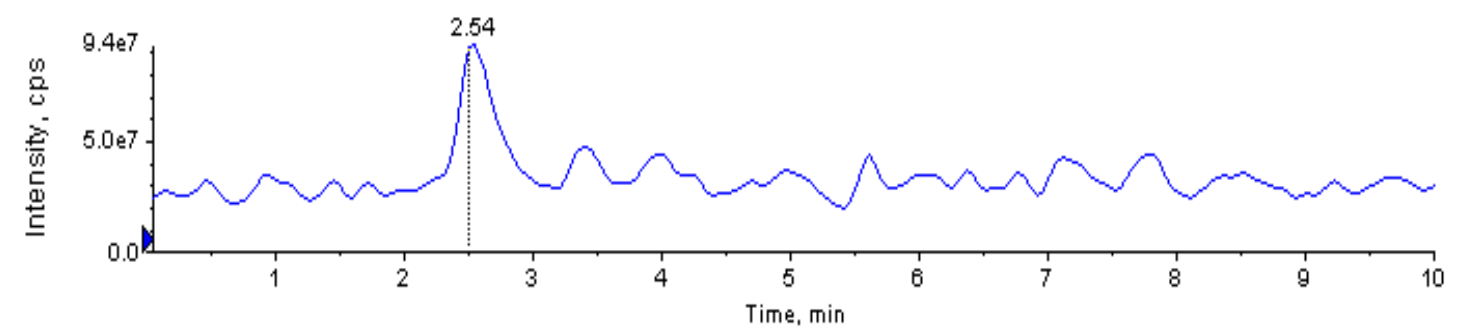

.Q1: 2.505 min from Sample 2 (SUC-2) of \#1SUCneg001TIC5050.wiff Max. 4.7e $7 \mathrm{cps}$.

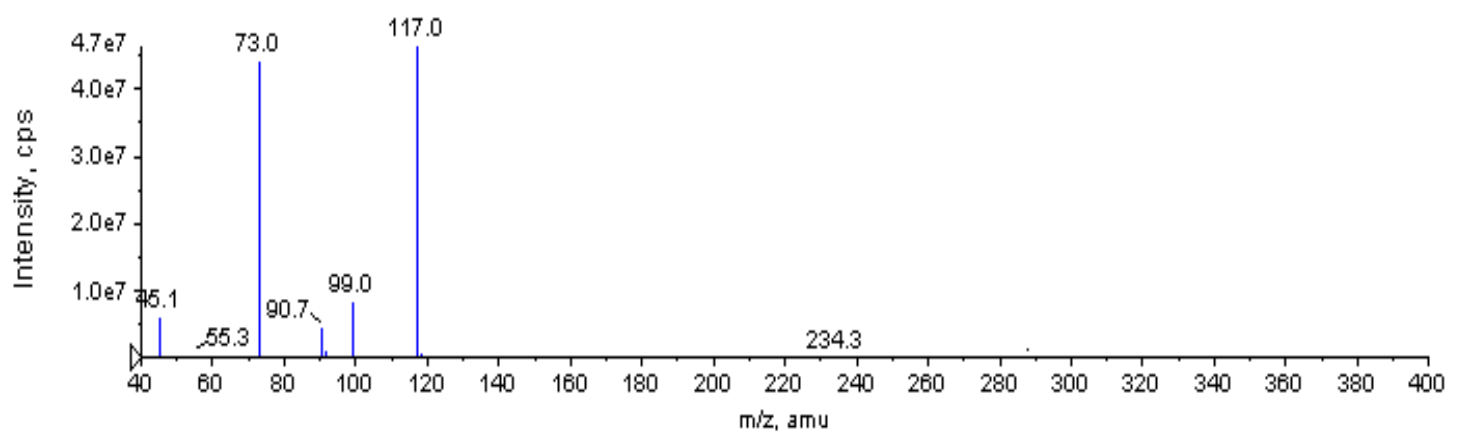

TIC of -01: from Sample 4 (SUC-4) of \#1SUCneg001center5050. wiiff Max. 1.1e7 cps.

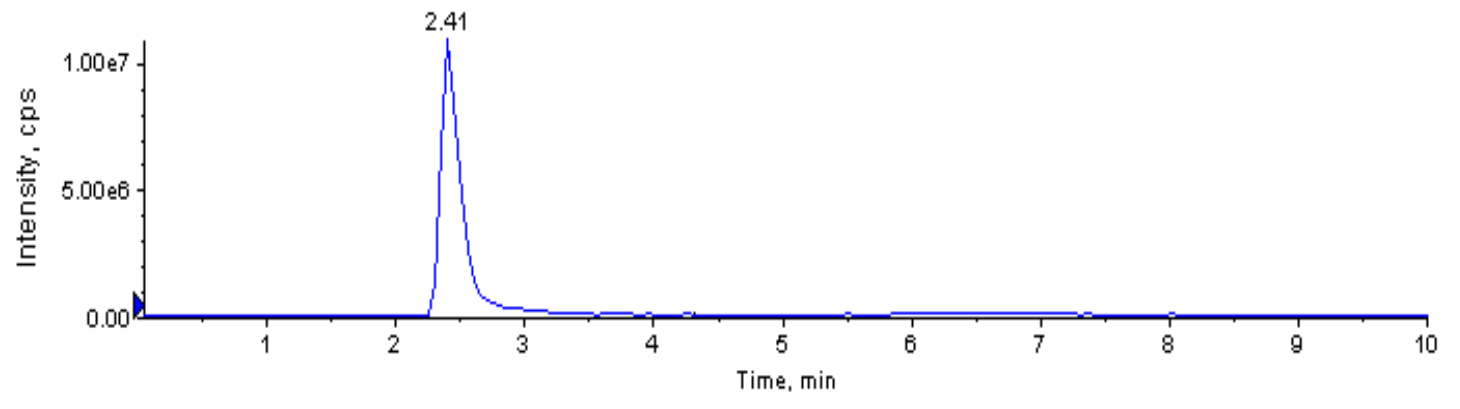

Figure 38. Succinic acid: total ion current, ESI (-) mass spectrum of molecular ion $(M-1)^{-1}=117$ amu, and SIM chromatogram on TDF C8 for 50 : 50 acetonitrile : water (at $\mathrm{pH}$ 6). 
Table 20. Retention times of organic acids on the TDF C8 column using LCMS

\begin{tabular}{|c|c|c|c|c|c|}
\hline \multicolumn{2}{|c|}{$\begin{array}{c}\text { Concentration } \\
(\%)\end{array}$} & \multicolumn{4}{|c|}{$\begin{array}{l}\text { Retention time } \\
\text { (min) }\end{array}$} \\
\hline $\begin{array}{l}\text { Acetonitrile } \\
(15 \mathrm{mM} \\
\text { Ammonium- } \\
\text { formate })\end{array}$ & $\begin{array}{l}\text { DI Water } \\
(15 \mathrm{mM} \\
\text { Ammonim- } \\
\text { formate })\end{array}$ & Maleic acid & $\begin{array}{l}\text { Fumaric } \\
\text { acid }\end{array}$ & $\begin{array}{l}\text { Succinic } \\
\text { acid }\end{array}$ & Citric acid \\
\hline 50 & 50 & 2.41 & 2.24 & 2.41 & 2.22 \\
\hline 60 & 40 & 2.48 & 2.36 & 2.55 & 2.32 \\
\hline 70 & 30 & 2.57 & 2.62 & 2.86 & 2.61 \\
\hline 80 & 20 & 2.73 & 3.66 & 3.82 & 3.89 \\
\hline 85 & 15 & 2.87 & 5.05 & 5.07 & 6.62 \\
\hline 90 & 10 & 3.00 & 9.97 & 8.78 & 19.15 \\
\hline
\end{tabular}

As given in Table 20, all the organic acids show good ANP retention as the acetonitrile concentration increases in the mobile phase compositions 50:50, 60:40, 70:30, 80:20, 85:15, and 90:10 acetonitrile:water $\mathrm{pH} \sim 6$ analyzed on the TDF C8 column. Citric acid is retained the most and maleic acid is retained the least which is explained by the fact that the higher the molecule polarity, the higher is the ANP retention when greater 


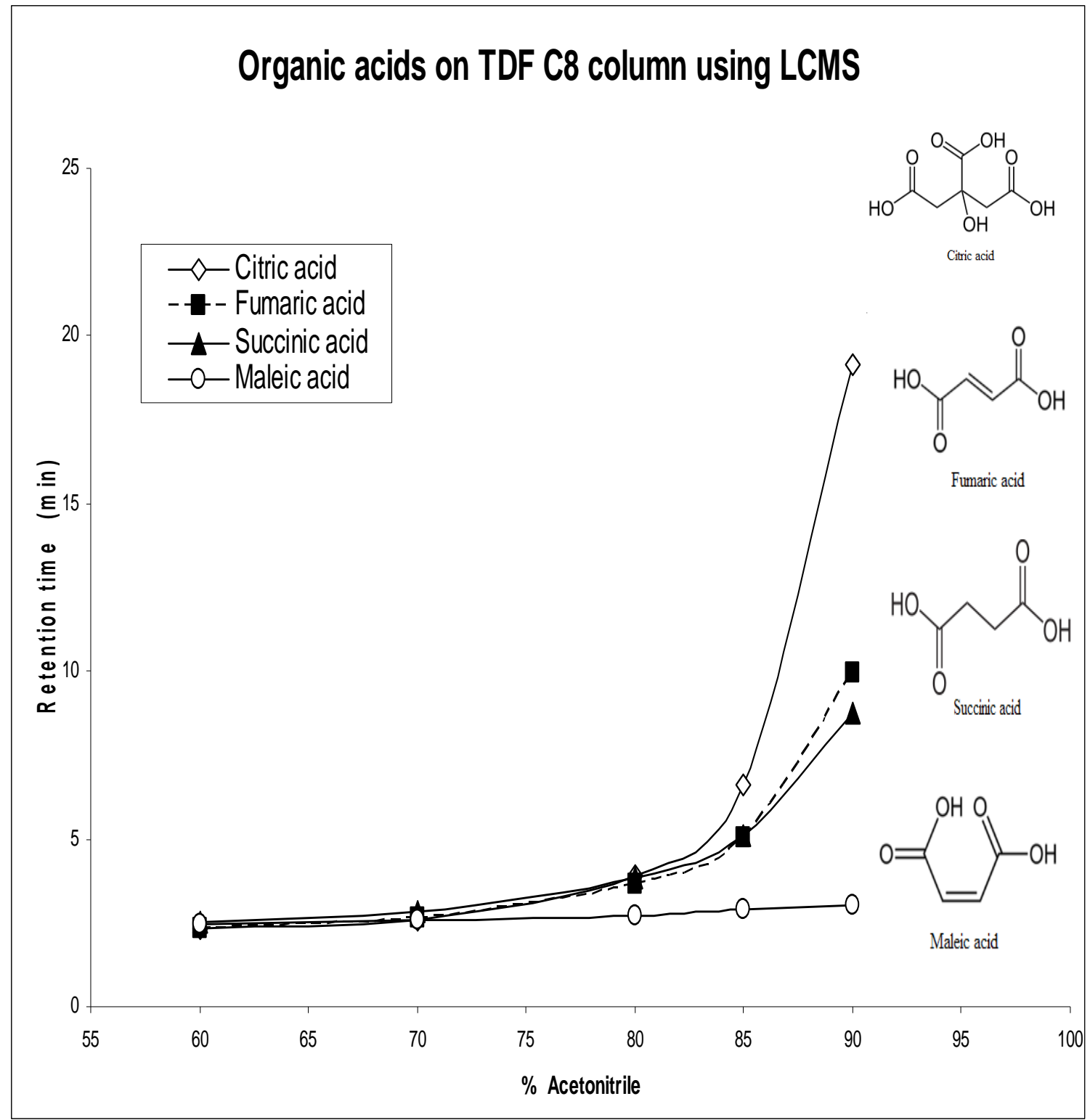

Figure 39. Retention map of organic acids on the TDF C8 column using LCMS.

amount of nonpolar solvent is run in the mobile phase. Figure 39 shows the characteristic retention maps on the TDF C8 column of all four organic acids as a function of the concentration of acetonitrile. These retention maps demonstrate excellent ANP retention 
of organic acids on the fluorinated TDF C8 column. Moreover, the distinct retention maps seen in the range of $80 \%$ to $90 \%$ acetonitrile concentration show the ability to provide a high degree of separation from one another if the analysis using a gradient is considered. Table 21 shows the retention time data of organic acids as the concentration of acetonitrile increases on the HDF C10 column and Figure 40 presents the related retention maps of the four organic acids. The trend of the ANP retention on HDF C10 is similar to that found on the TDF C8 column.

Table 21. Retention times of organic acids on the HDF C10 column using LCMS

\begin{tabular}{|c|c|c|c|c|c|}
\hline \multicolumn{2}{|c|}{$\begin{array}{c}\text { Concentration } \\
(\%)\end{array}$} & \multicolumn{4}{|c|}{$\begin{array}{l}\text { Retention time } \\
\text { (min) }\end{array}$} \\
\hline $\begin{array}{c}\text { Acetonitrile } \\
+ \\
(15 \mathrm{mM} \\
\text { Ammonium- } \\
\text { formate })\end{array}$ & $\begin{array}{c}\text { DI Water } \\
+ \\
(15 \mathrm{mM} \\
\text { Ammonim- } \\
\text { formate })\end{array}$ & Maleic acid & $\begin{array}{l}\text { Fumaric } \\
\text { acid }\end{array}$ & $\begin{array}{l}\text { Succinic } \\
\text { acid }\end{array}$ & Citric acid \\
\hline 50 & 50 & 2.37 & 2.18 & 2.35 & 2.15 \\
\hline 60 & 40 & 2.41 & 2.26 & 2.42 & 2.24 \\
\hline 70 & 30 & 2.52 & 2.56 & 2.77 & 2.50 \\
\hline 80 & 20 & 2.69 & 3.38 & 3.69 & 3.66 \\
\hline 85 & 15 & 2.79 & 4.97 & 5.02 & 6.70 \\
\hline 90 & 10 & 3.00 & 9.98 & 8.67 & 18.94 \\
\hline
\end{tabular}




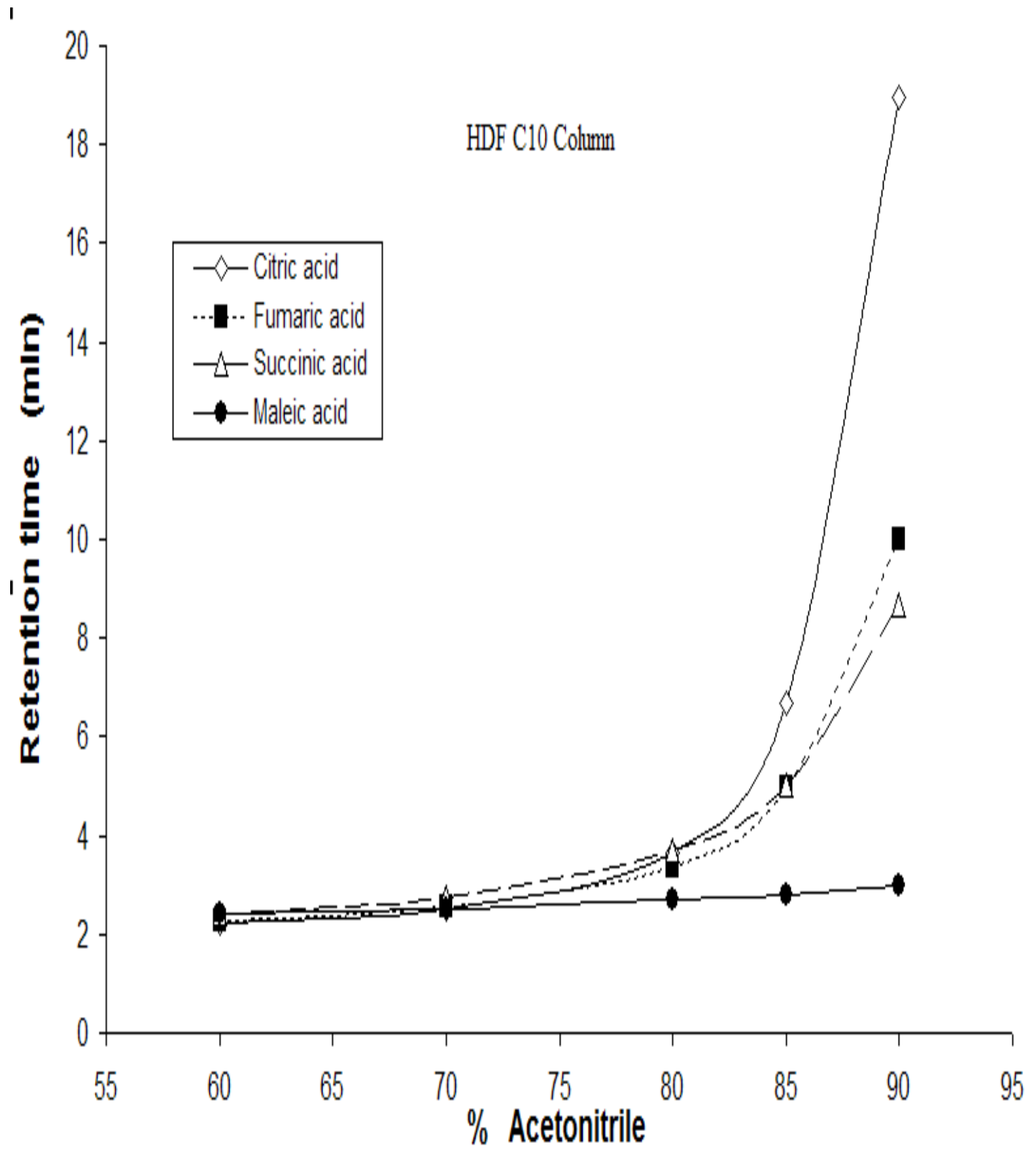

I

Figure 40. Retention map of organic acids on the HDF C10 column using LCMS. 


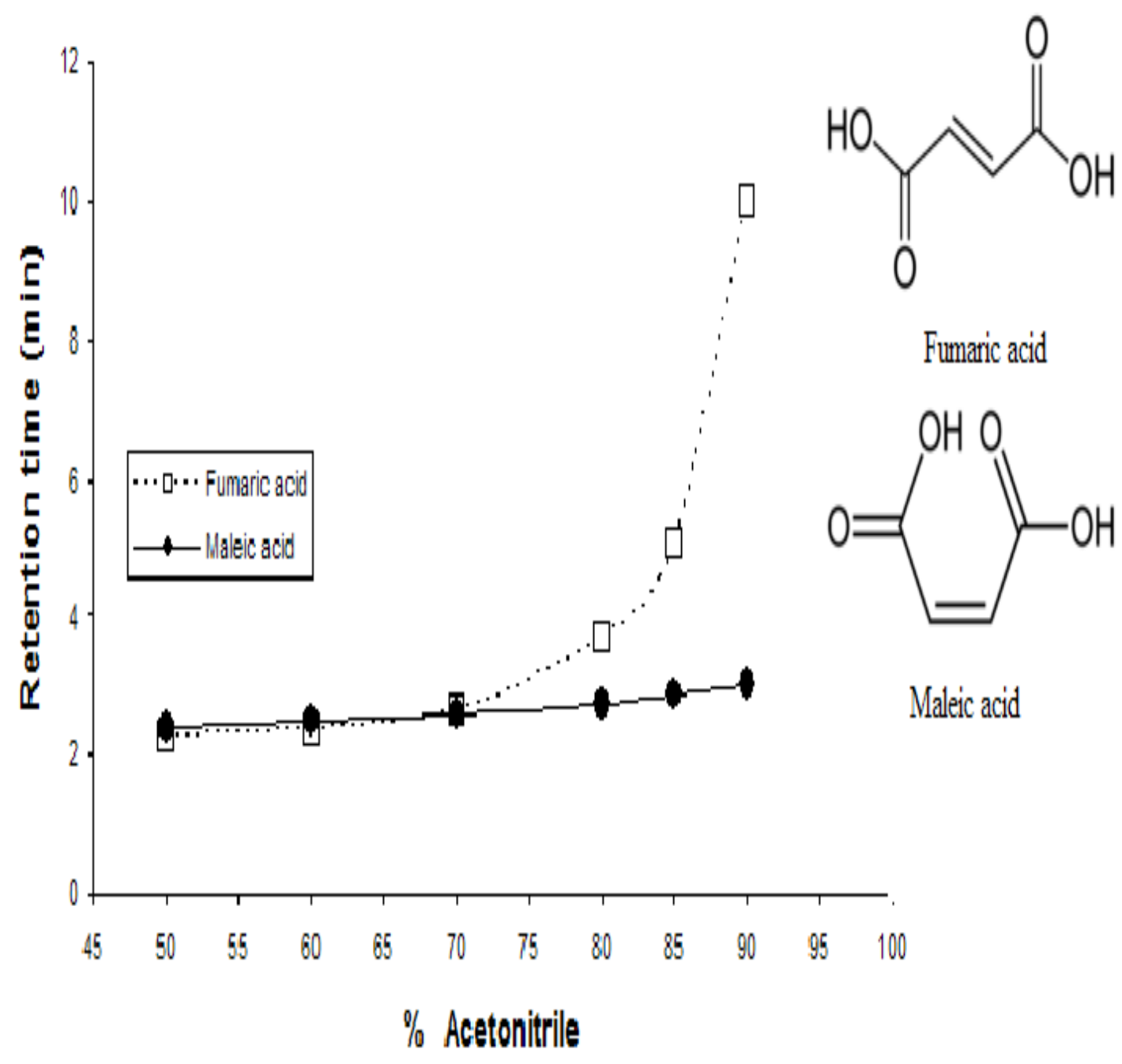

Figure 41. Retention map of isobaric fumaric acid and maleic acid on the TDF C8 column using LCMS.

Figure 41 shows the retention map of isobaric maleic acid and fumaric acid analyzed on the TDF C8 column. The only difference between these two conformers is their spatial arrangements which make fumaric acid more polar contributing to higher ANP retention than that of maleic acid as the $\%$ of acetonitrile increases. 


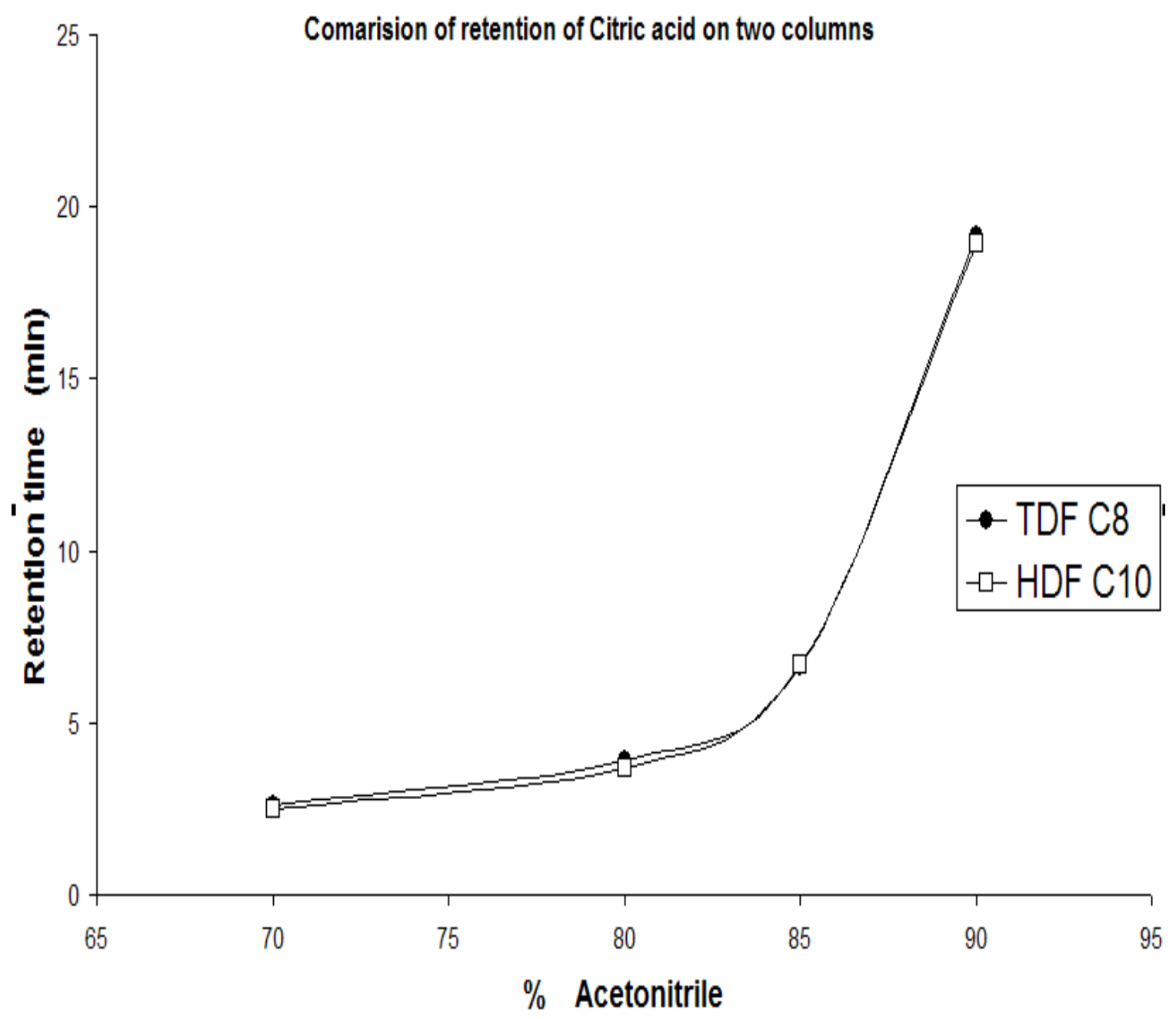

Figure 42. Comparison of retention maps of citric acid on the TDF C8 and HDF C10 columns.

Figure 42 is the comparison of the retention maps of citric acid on the TDF C8 and HDF C10 columns. The exact overlap of the retention maps implies that in this case both the underlying silica hydride layer and the chemically attached fluorinated bonded moiety play an equal role in the ANP retention of citric acid. 


\subsection{Carbohydrates}

The ANP behavior of the TDF C8 and HDF C10 columns using LC-MS was further assessed for glucose, fructose and ribose as monosaccharide probes and sucrose, lactose, maltose, trehalose and turanose as disaccharide probes. All these carbohydrate solutes are highly polar and not easy to retain using classic reversed phase chromatography. LCMS is the most suitable detector for these compounds and this system is compatible with the use of $15 \mathrm{mM}$ ammonium formate that gives higher ANP retention on the columns. In parallel, the nonpolar cellobiose octaacetate disaccharide was also analyzed to check the reversed phase retention behavior of the columns.

Table 19 (C) lists the molecular weight of the carbohydrates that have been analyzed and the $\mathrm{m} / \mathrm{z}$ values of their sodium adduct used for obtaining the SIM chromatograms. The $[\mathrm{M}+\mathrm{Na}]^{+1}$ adducts of carbohydrates were scanned using the ESI positive ion mode of detection. The mobile phase flow rate was set to $0.4 \mathrm{~mL} / \mathrm{min}$ for all the carbohydrate solutes analyzed using LC-MS.

\subsubsection{Monosaccharides}

The monosaccharides glucose, fructose, and ribose were analyzed on both the TDF C8 and HDF C10 columns and they displayed ANP behavior with an increase of acetonitrile concentration. For the retention analysis of glucose and fructose, the sample was dissolved to make a $1 \mathrm{mg} / \mathrm{mL}$ solution using 50:50 acetonitrile:water at $\mathrm{pH} \sim 6$ using $15 \mathrm{mM}$ ammonium formate. The injection volume was optimized to $0.5 \mu \mathrm{L}$ and the threshold was set to 50 for both columns. 
Figure 43 is an example of MS plots of D-glucose analyzed on the TDF C8 column. The top frame is the TIC chromatogram of glucose obtained for 70:30 acetonitrile:water ( $\mathrm{pH}$ ). The middle frame is the mass spectrum of glucose showing the $\mathrm{m} / \mathrm{z} 203.1 \mathrm{amu}$ of $[\mathrm{M}+\mathrm{Na}]^{+1}$ sodium adduct ion. The last frame is the SIM chromatogram of the glucose sodium adduct ion with a mobile phase consisting of 95:05 acetonitrile:water $(\mathrm{pH} 6)$. The SIM chromatogram shows a very symmetrical ANP retention peak for glucose at 6.55 minutes on the TDF C 8 column. Similarly, retention times for glucose were recorded using 50:50, 60:40, 70:30, 80:20, 90:10, and 95:05 acetonitrile:water $\mathrm{pH} \sim 6$ mobile phase system and tabulated as shown in Table 22.

D-Fructose has the same molecular weight as D-glucose, hence the same value of the sodium adduct ion $(\mathrm{m} / \mathrm{z} 203.1 \mathrm{amu})$ was observable and used to scan for the SIM chromatograms. However, in the case of D-ribose, the sodium adduct ion of $173.13 \mathrm{amu}$ was scanned to get the SIM peaks. Table 22 also lists the SIM retention data monitored for fructose and ribose as the acetonitrile concentration increases.

For the retention analysis of ribose, the sample solution was made as a $1 \mathrm{mg} / \mathrm{mL}$ solution in 50:50 acetonitrile:water $\mathrm{pH} \sim 6$ mobile phase and an injection volume of 5.0 $\mu \mathrm{L}$ was used to get sharp symmetric peaks with the threshold set to 50 . Peak splitting was observed at 70:30 and 80:20 acetonitrile:water $\mathrm{pH} \sim 6$ mobile phase compositions for ribose on both columns. This splitting might be overcome if the sample solution is made in the same composition as the mobile phase running. However, it is worthwhile to mention that for 90:10 and 95:05 acetonitrile:water $\mathrm{pH} \sim 6$, very sharp peaks with good signal to noise were observed for d-ribose. 
TIC of +03: from Sample 1 (GSE-1) of \#1GSEpoS003TIC7030.witf

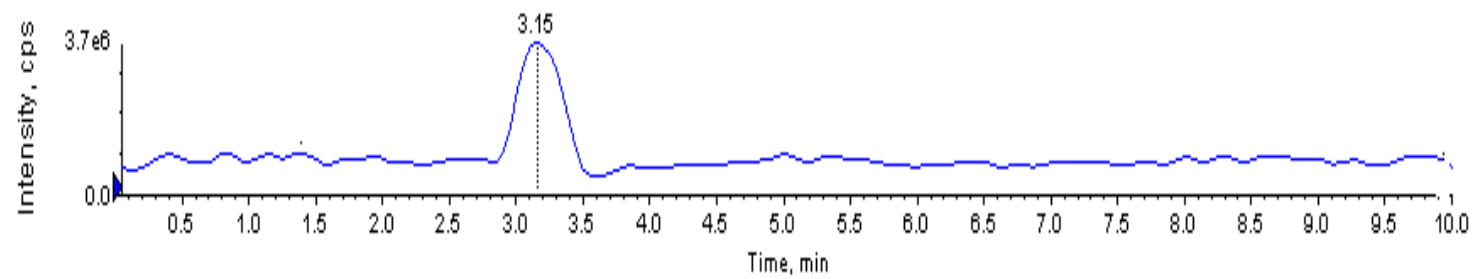

+03: 3.156 min from Sample 1 (GSE-1) of \#1GSEpos003TIC7030.wift

Max. $2.2 .6 \mathrm{cps}$

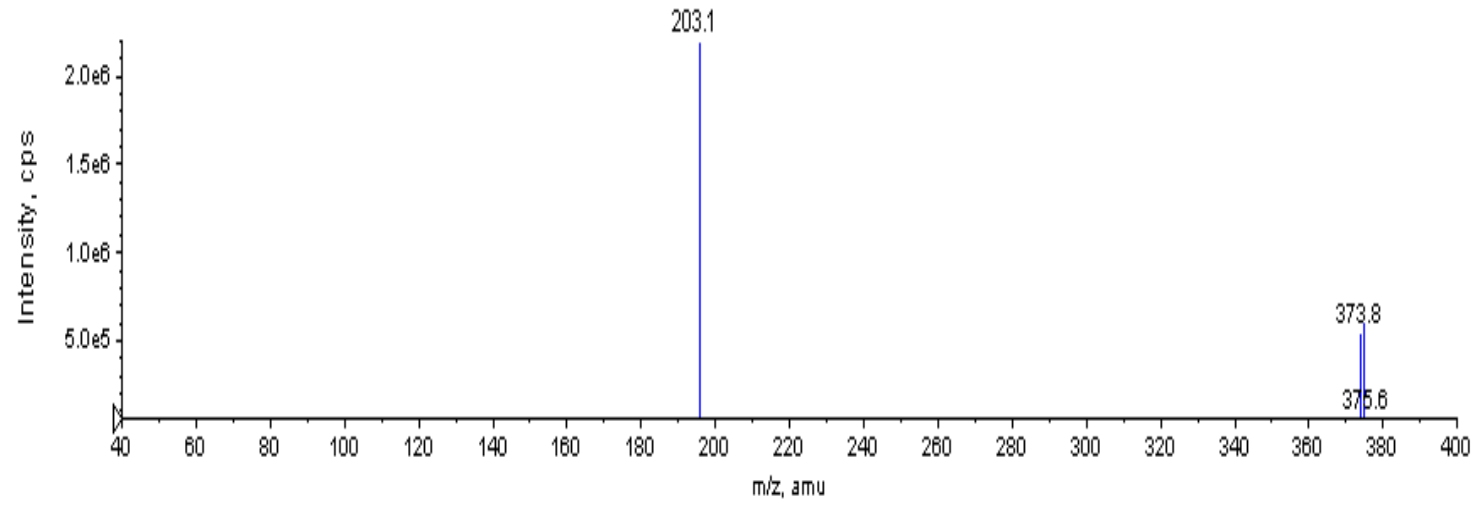

TIC of +03: from Sample 1 (GSE-1) of \#1GSEpos003center 9505 . witf

Max. 1.1.e5 cps.

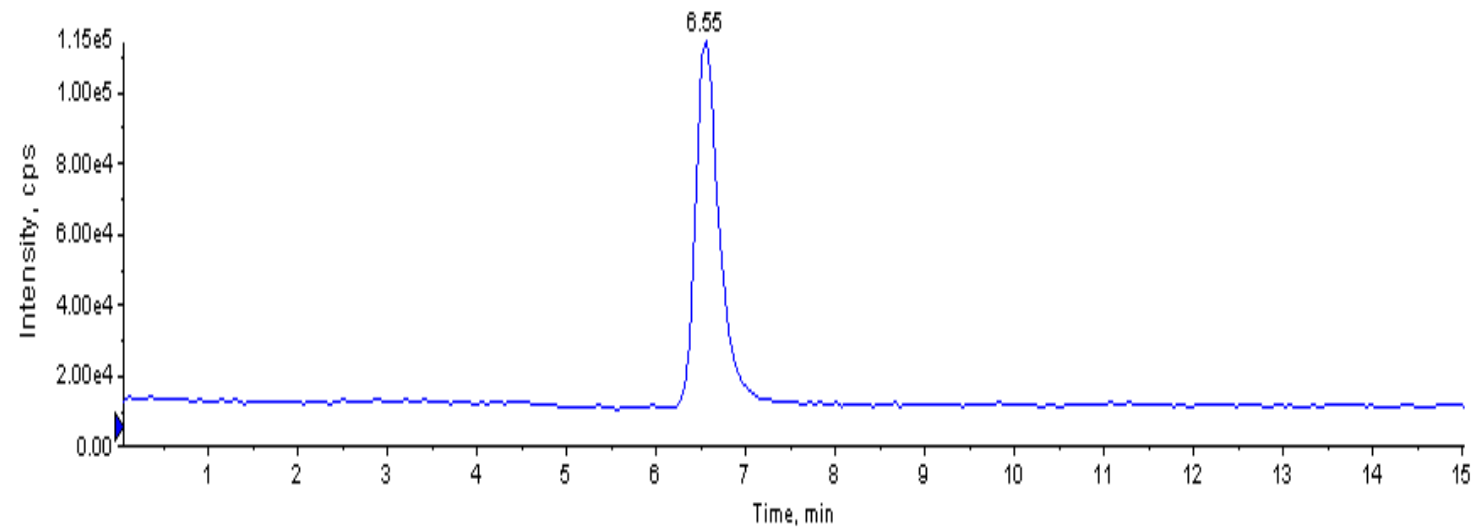

Figure 43. Glucose: total ion current, ESI (+) mass spectrum of sodium adduct $(\mathrm{M}+\mathrm{Na})^{+1}=203.1 \mathrm{amu}$, and SIM chromatogram on TDF C8 for 95: 05 acetonitrile : water (at $\mathrm{pH} 6$ ). 
Table 22. Retention times of monosaccharides on the TDF C8 column using LCMS

\begin{tabular}{|c|c|c|c|c|}
\hline \multicolumn{2}{|c|}{$\begin{array}{c}\text { Concentration } \\
(\%)\end{array}$} & \multicolumn{3}{|c|}{$\begin{array}{l}\text { Retention time } \\
\text { (min) }\end{array}$} \\
\hline $\begin{array}{c}\text { Acetonitrile } \\
+ \\
(15 \mathrm{mM} \\
\text { Ammonium- } \\
\text { formate })\end{array}$ & $\begin{array}{c}\text { DI Water } \\
+ \\
(15 \mathrm{mM} \\
\text { Ammonim- } \\
\text { formate })\end{array}$ & D-Ribose & D-Glucose & D-Fructose \\
\hline 50 & 50 & 3.07 & 2.92 & 2.96 \\
\hline 60 & 40 & 3.16 & 2.96 & 3.02 \\
\hline 70 & 30 & 3.35 & 3.15 & 3.22 \\
\hline 80 & 20 & 3.60 & 3.65 & 3.70 \\
\hline 90 & 10 & 4.20 & 4.83 & 4.76 \\
\hline 95 & 05 & 4.70 & 6.55 & 6.25 \\
\hline
\end{tabular}

As depicted in Table 22, all three monosaccharide solutes show very good ANP retention with increasing acetonitrile concentration on the TDF C8 column. The retention of ribose is somewhat less than that of glucose and fructose. Figure 44, Figure 45, and Figure 46 show an individual ANP retention map of d-glucose, d-fructose, and dribose respectively on the TDF C8 column. They are plotted together in a single graph for comparative purposes as shown in Figure 47. 


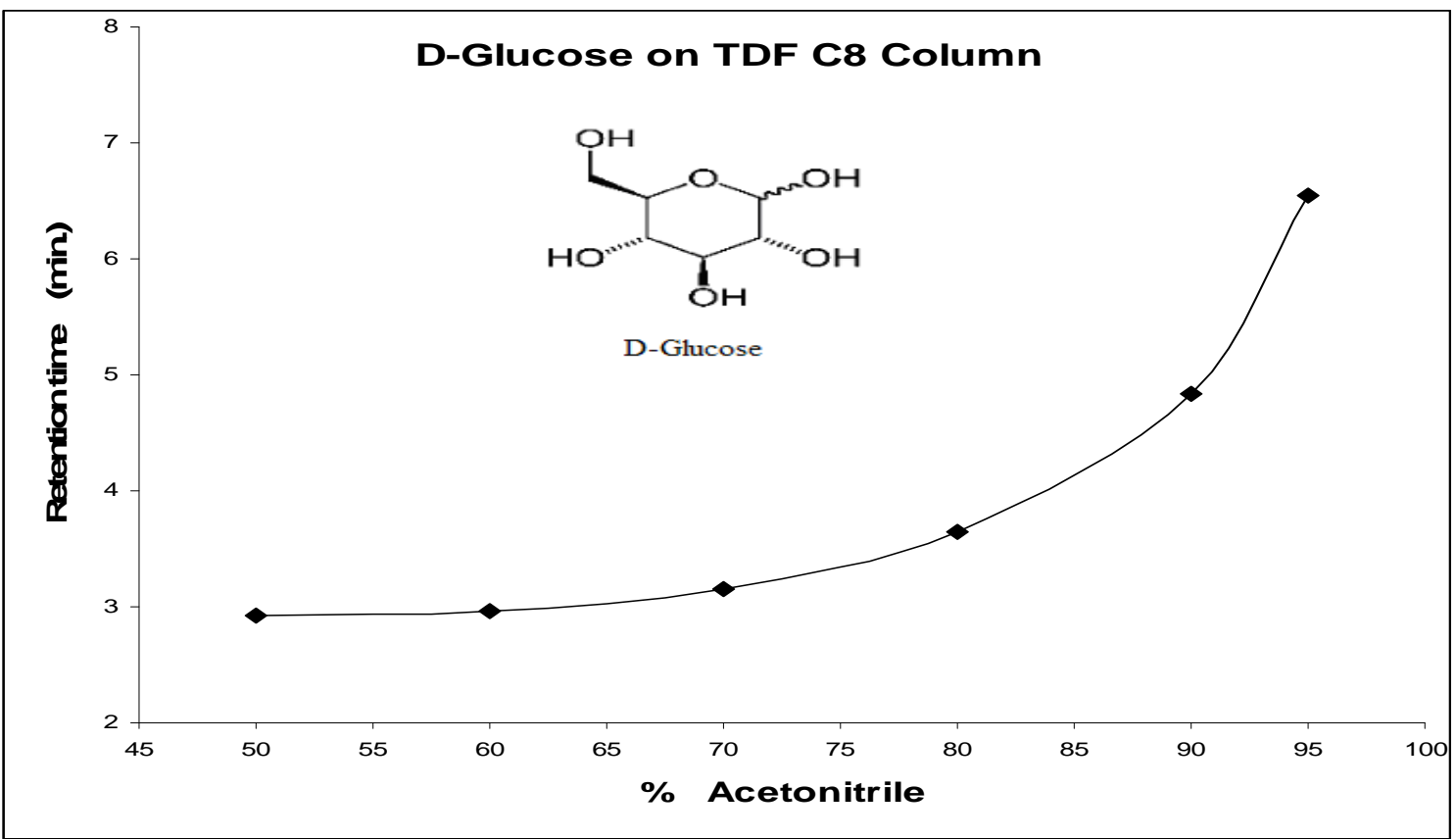

Figure 44. ANP retention of D-glucose on the TDF C8 column.

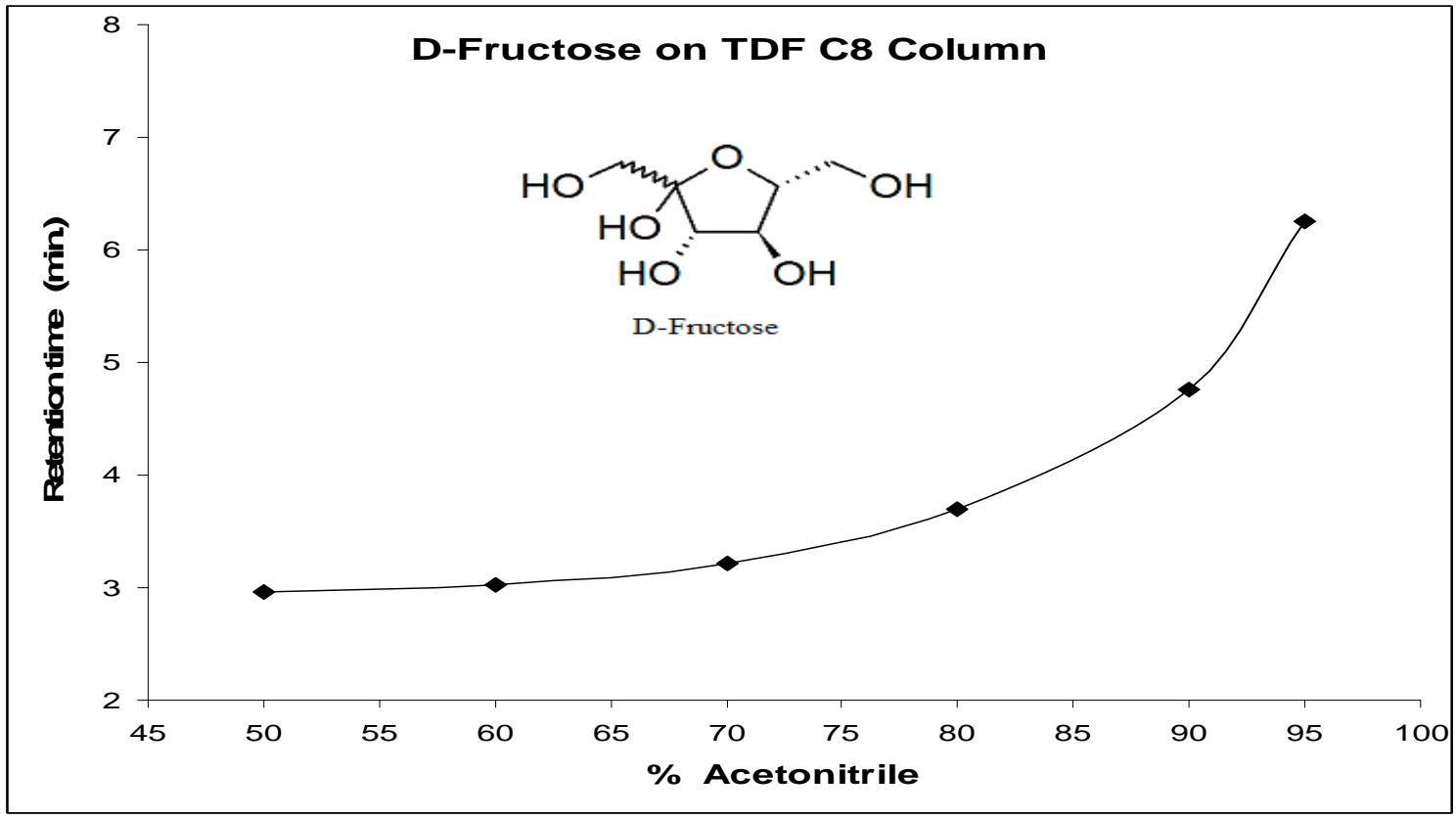

Figure 45. ANP retention of D-fructose on the TDF C8 column. 


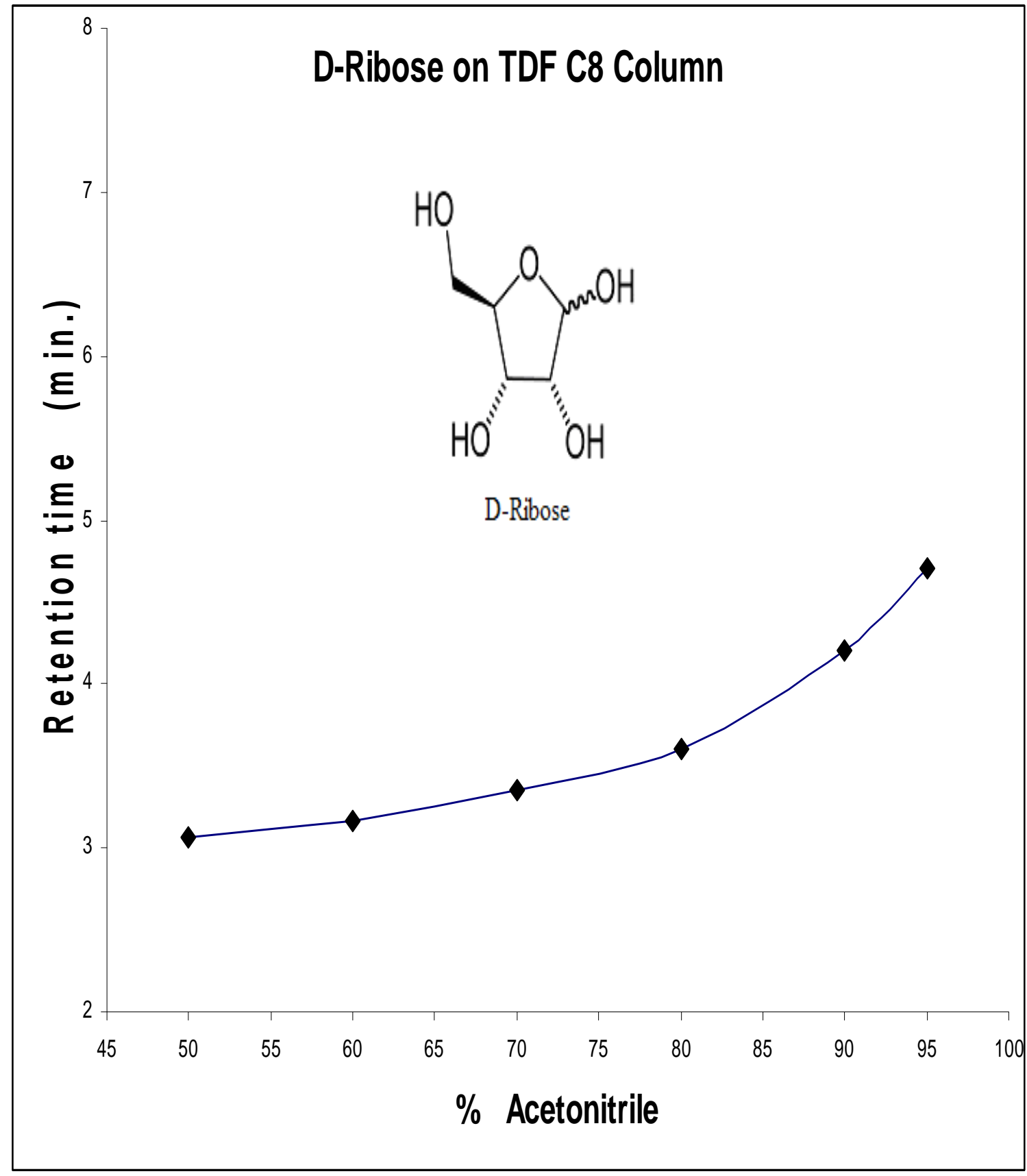

Figure 46. ANP retention of D-ribose on the TDF C8 column. 


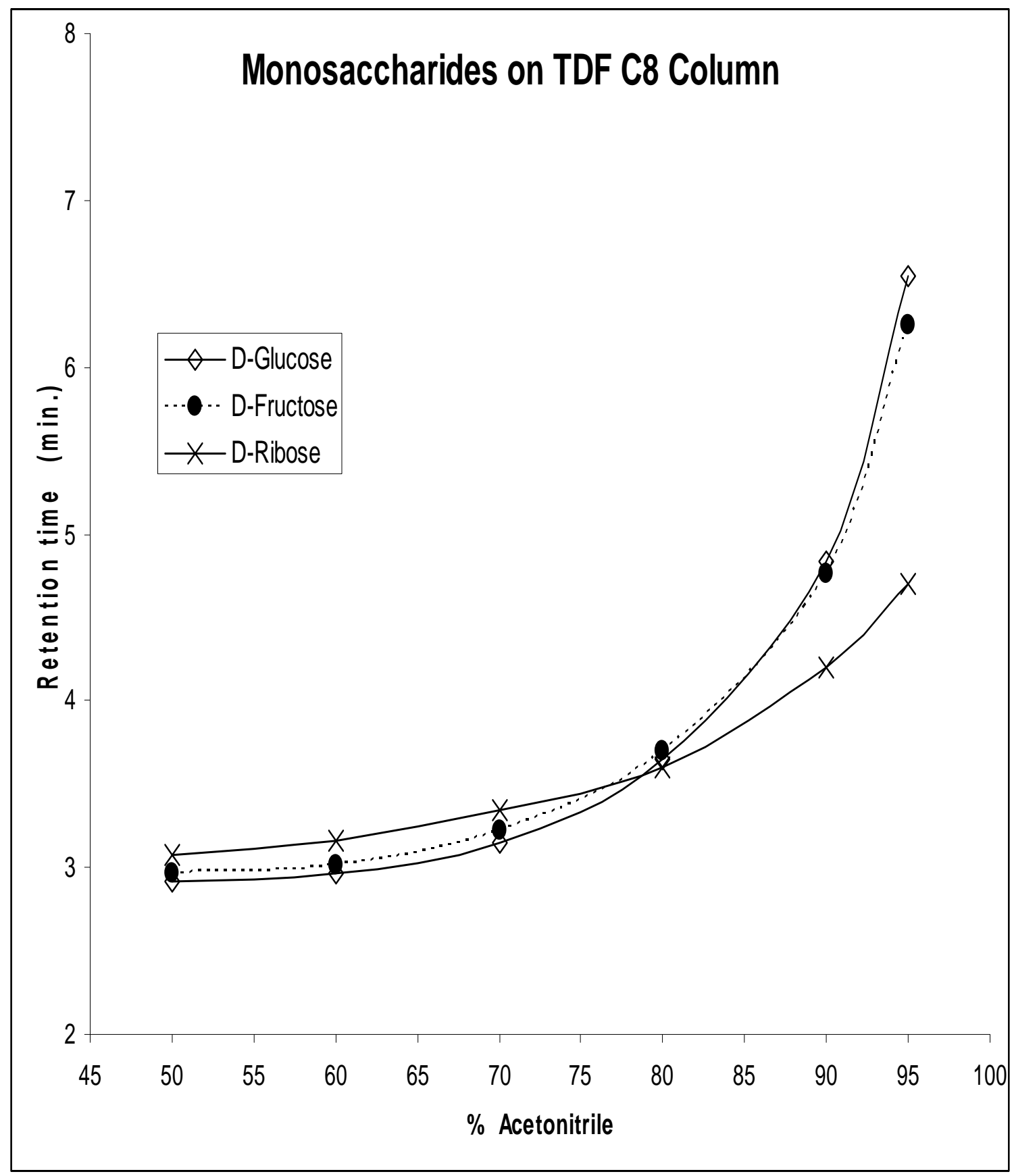

Figure 47. Retention map of monosaccharides on the TDF C8 column. 
Table 23. Retention times of monosaccharides on the HDF C10 column using LCMS

\begin{tabular}{|c|c|c|c|c|}
\hline \multicolumn{2}{|c|}{$\begin{array}{c}\text { Concentration } \\
\text { (\%) }\end{array}$} & \multicolumn{3}{c|}{$\begin{array}{c}\text { Retention time } \\
\text { (min) }\end{array}$} \\
\hline $\begin{array}{c}\text { Acetonitrile } \\
+ \\
(15 \mathrm{mM} \\
\text { Ammonium- } \\
\text { formate) }\end{array}$ & $\begin{array}{c}\text { DI Water } \\
+ \\
(15 \mathrm{mM} \\
\text { Ammonim- } \\
\text { formate })\end{array}$ & D-Ribose & D-Glucose & D-Fructose \\
\hline 50 & 50 & 2.99 & $2 . .86$ & 2.92 \\
\hline 60 & 40 & 3.16 & 2.95 & 3.00 \\
\hline 70 & 30 & 3.16 & 3.17 & 3.20 \\
\hline 80 & 20 & 3.47 & 3.56 & 3.65 \\
\hline 90 & 10 & 4.05 & 4.75 & 4.70 \\
\hline 95 & 05 & 4.61 & 6.38 & 6.12 \\
\hline
\end{tabular}

Table 23 lists their retention times and in Figure 48 are plots of their ANP retention maps on the HDF C10 column under similar conditions. If one compares the ANP retention on the TDF C8 and HDF C10 columns, the plots of each individual analyte would completely overlap indicating equal contribution of the hydride layer and fluorinated moieties in the retention mechanism. 


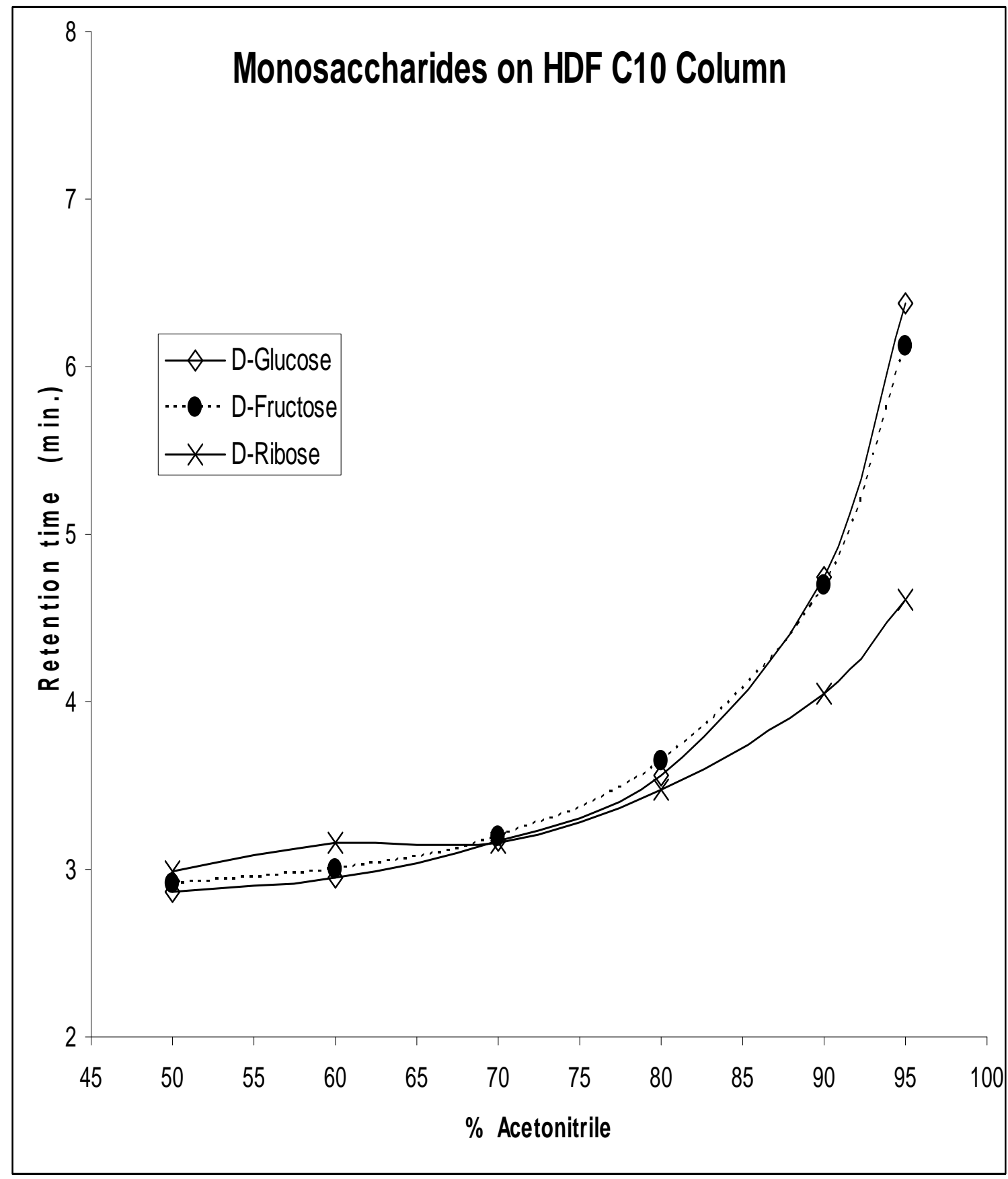

Figure 48. Retention map of monosaccharides on the HDF C10 column. 


\subsubsection{Disaccharides}

The disaccharides sucrose, lactose, maltose, trehalose, and turanose are polar molecules and each one has the same molecular weight i.e., $342.30 \mathrm{amu}$. Their mass spectra yielded the exact same value of $\mathrm{m} / \mathrm{z} 365.30 \mathrm{amu}$ for their respective sodium

Table 24. Retention times of disaccharides on the TDF C8 column using LCMS

\begin{tabular}{|c|c|c|c|c|}
\hline \multicolumn{2}{|c|}{$\begin{array}{c}\text { Concentration } \\
\text { (\%) }\end{array}$} & \multicolumn{3}{|c|}{$\begin{array}{c}\text { Retention time } \\
\text { (min) }\end{array}$} \\
\hline $\begin{array}{c}\text { Acetonitrile } \\
+ \\
(15 \mathrm{mM} \\
\text { Ammonium- } \\
\text { formate })\end{array}$ & $\begin{array}{c}\text { DI Water } \\
+ \\
(15 \mathrm{mM} \\
\text { Ammonim- } \\
\text { formate })\end{array}$ & Sucrose & Lactose & Maltose \\
\hline 50 & 50 & 2.86 & 2.91 & 2.85 \\
\hline 60 & 40 & 2.93 & 2.91 & 2.94 \\
\hline 70 & 30 & 3.15 & 3.20 & 3.19 \\
\hline 80 & 20 & 3.70 & 3.90 & 3.78 \\
\hline 90 & 10 & 5.54 & 6.19 & 5.62 \\
\hline 95 & 05 & 8.85 & 9.27 & 10.67 \\
\hline
\end{tabular}


adduct ions. Hence, $\mathrm{m} / \mathrm{z}$ of $365.30 \mathrm{amu}$ was monitored to obtain the SIM of all these disaccharides using the compositions of 50:50, 60:40, 70:30, 80:20, 90:10, and 95:05 acetonitrile:water at $\mathrm{pH} \sim 6$ mobile phase conditions and their ANP retention times were tabulated for both columns.

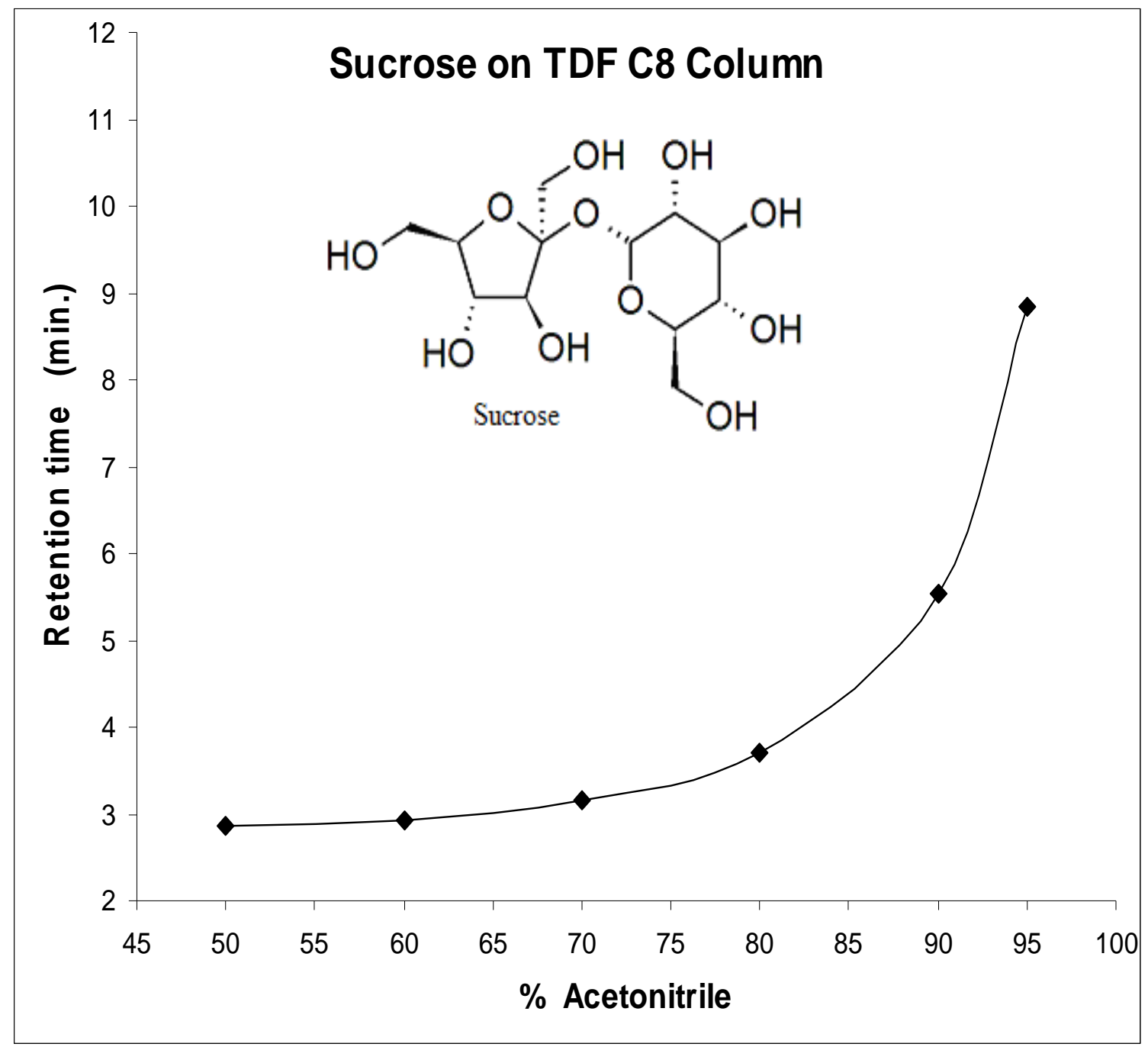

Figure 49. ANP retention of sucrose on the TDF C8 column. 


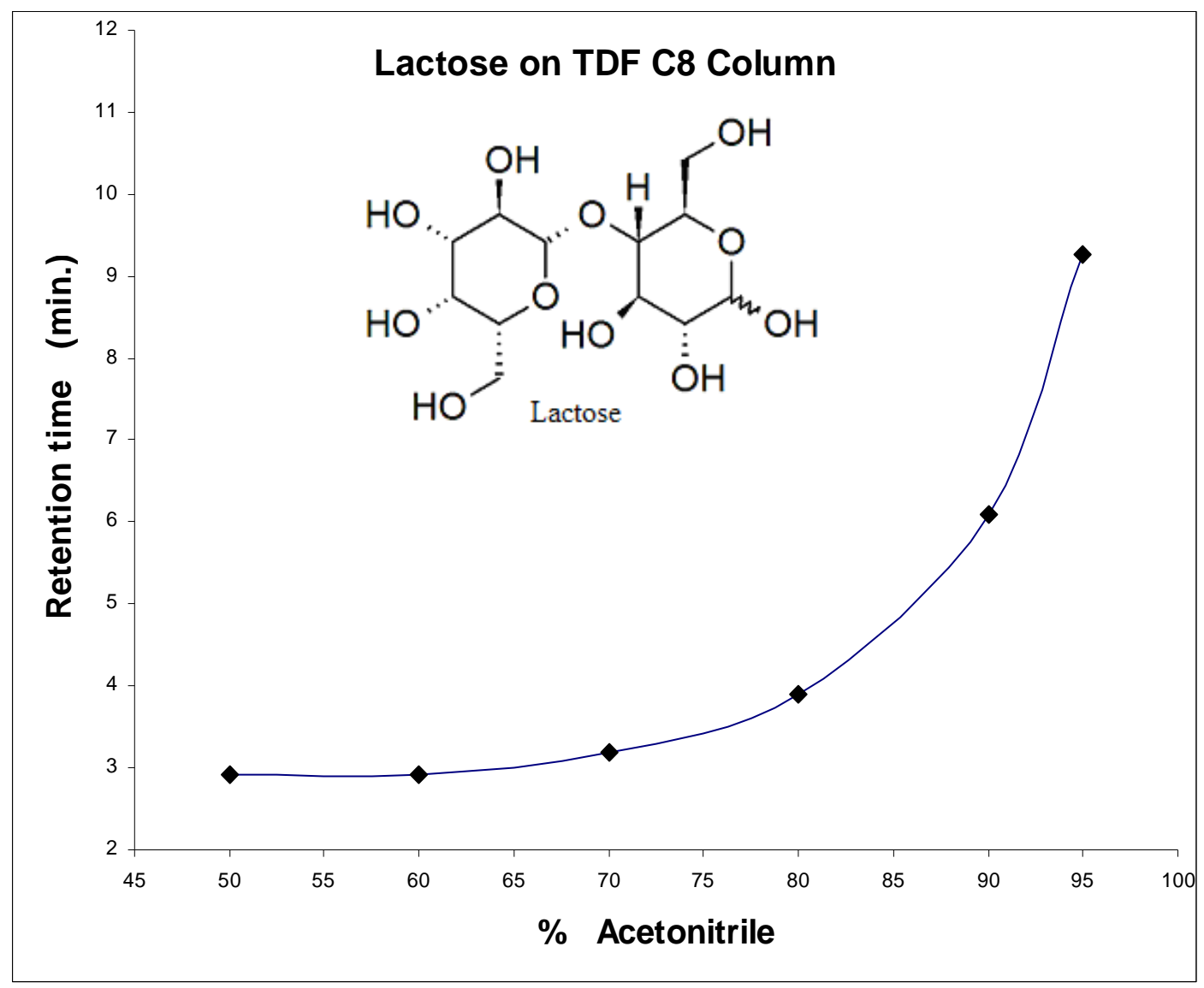

Figure 50. ANP retention of lactose on the TDF C8 column.

For the MS analysis of sucrose and lactose, a $0.5 \mu \mathrm{L}$ injection volume of a $1 \mathrm{mg} / \mathrm{mL}$ sample solution was used on both of the columns to produce sharp symmetric peaks. Whereas for maltose, the use of a $2.0 \mu \mathrm{L}$ injection volume of a $1 \mathrm{mg} / \mathrm{mL}$ sample solution resulted in good efficiency symmetric peaks. The threshold value was adjusted to 50 for all the analyses. Table 24 lists the retention times observed on the TDF C8 column and Table 25 gives the retention times observed on the HDF C10 column for sucrose, lactose, and maltose with increasing concentration of acetonitrile at $\mathrm{pH} \sim 6$ mobile phase conditions. Figure 49, Figure 50, and Figure 51 show the individual ANP 
retention maps of sucrose, lactose, and maltose respectively on the TDF C8 column. All of the retention maps are overlaid on the same plot as shown in Figure 52 for comparative understanding of the ANP retention of these solutes on the TDF C8 column. In parallel, the retention maps of sucrose, lactose, and maltose analyzed on the HDF C10 column are plotted all together as shown in Figure 53.

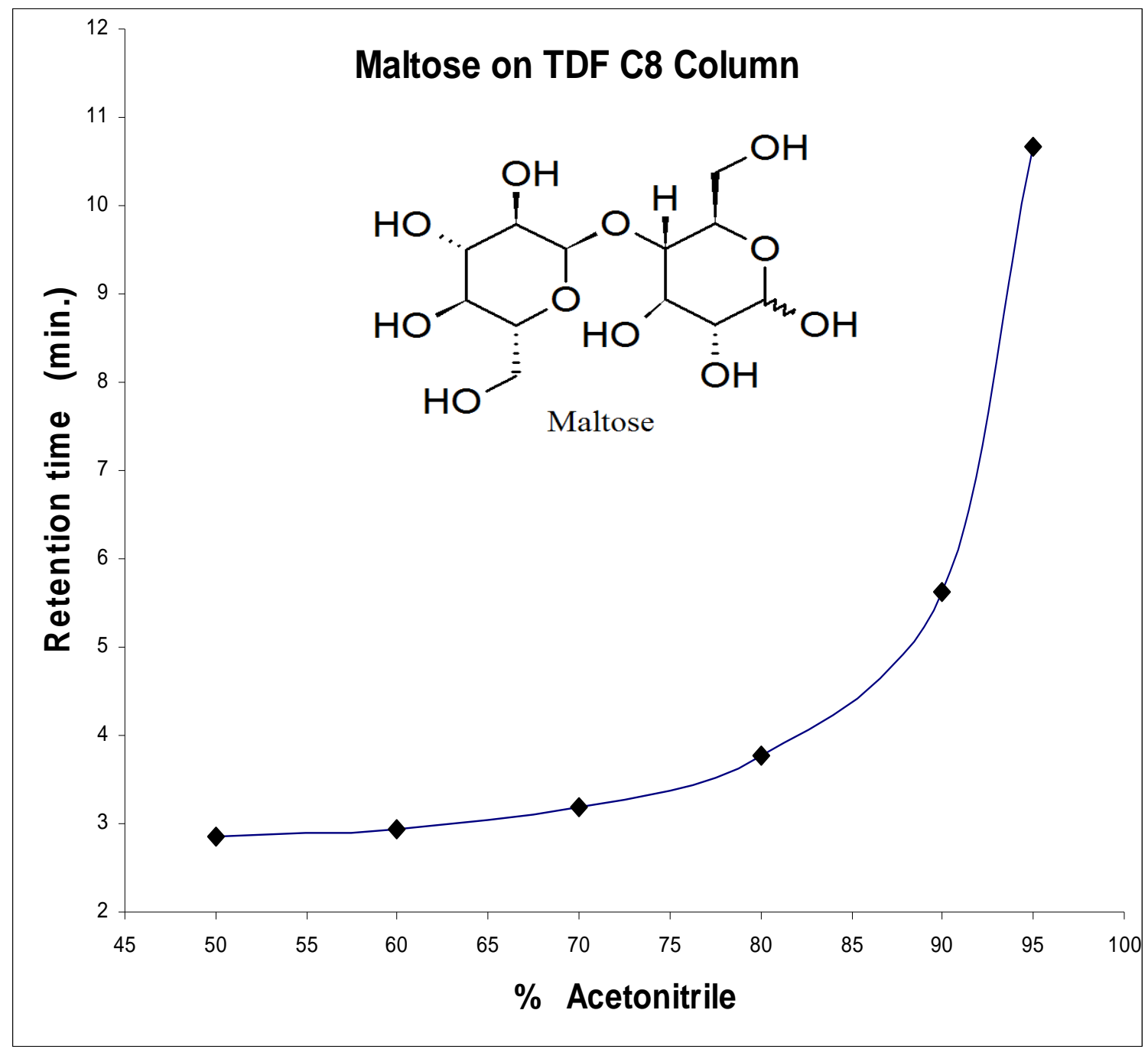

Figure 51. ANP retention of maltose on the TDF C8 column. 


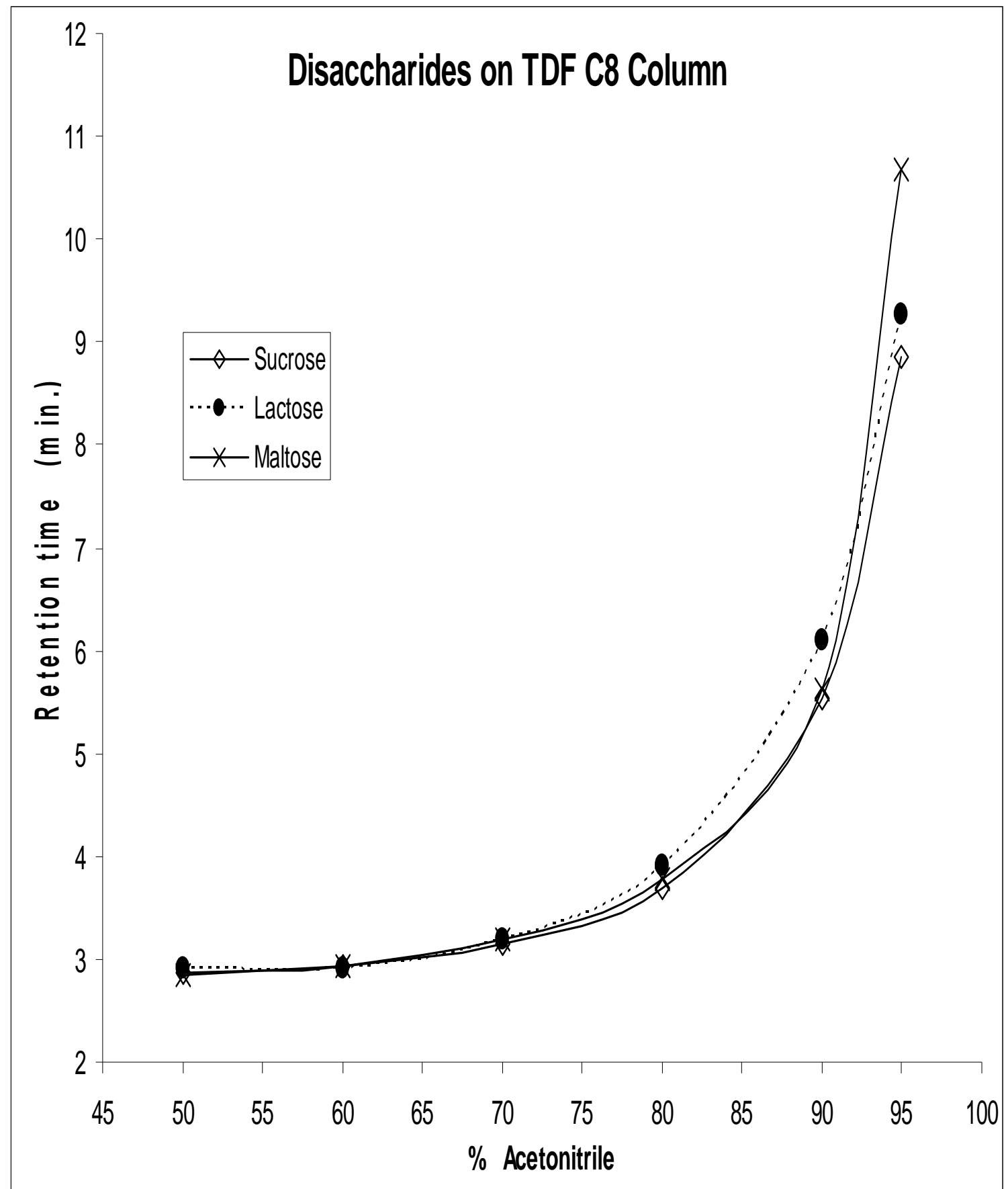

Figure 52. Retention map of disaccharides on the TDF C8 column. 
Table 25. Retention times of disaccharides on the HDF C10 column using LCMS

\begin{tabular}{|c|c|c|c|c|}
\hline \multicolumn{2}{|c|}{$\begin{array}{c}\text { Concentration } \\
\text { (\%) }\end{array}$} & \multicolumn{3}{|c|}{$\begin{array}{c}\text { Retention time } \\
\text { (min) }\end{array}$} \\
\hline $\begin{array}{c}\text { Acetonitrile } \\
+ \\
(15 \mathrm{mM} \\
\begin{array}{c}\text { Ammonium- } \\
\text { formate) }\end{array}\end{array}$ & $\begin{array}{c}\text { DI Water } \\
+ \\
(15 \mathrm{mM} \\
\text { Ammonim } \\
\text { - formate) }\end{array}$ & Sucrose & Lactose & Maltose \\
\hline 50 & 50 & 2.77 & 2.82 & 2.80 \\
\hline 60 & 40 & 2.86 & 2.88 & 2.91 \\
\hline 70 & 30 & 3.10 & 3.12 & 3.10 \\
\hline 80 & 20 & 3.66 & 3.87 & 3.67 \\
\hline 90 & 10 & 5.32 & 6.09 & 5.57 \\
\hline 95 & 05 & 8.48 & 10.62 & 9.15 \\
\hline
\end{tabular}




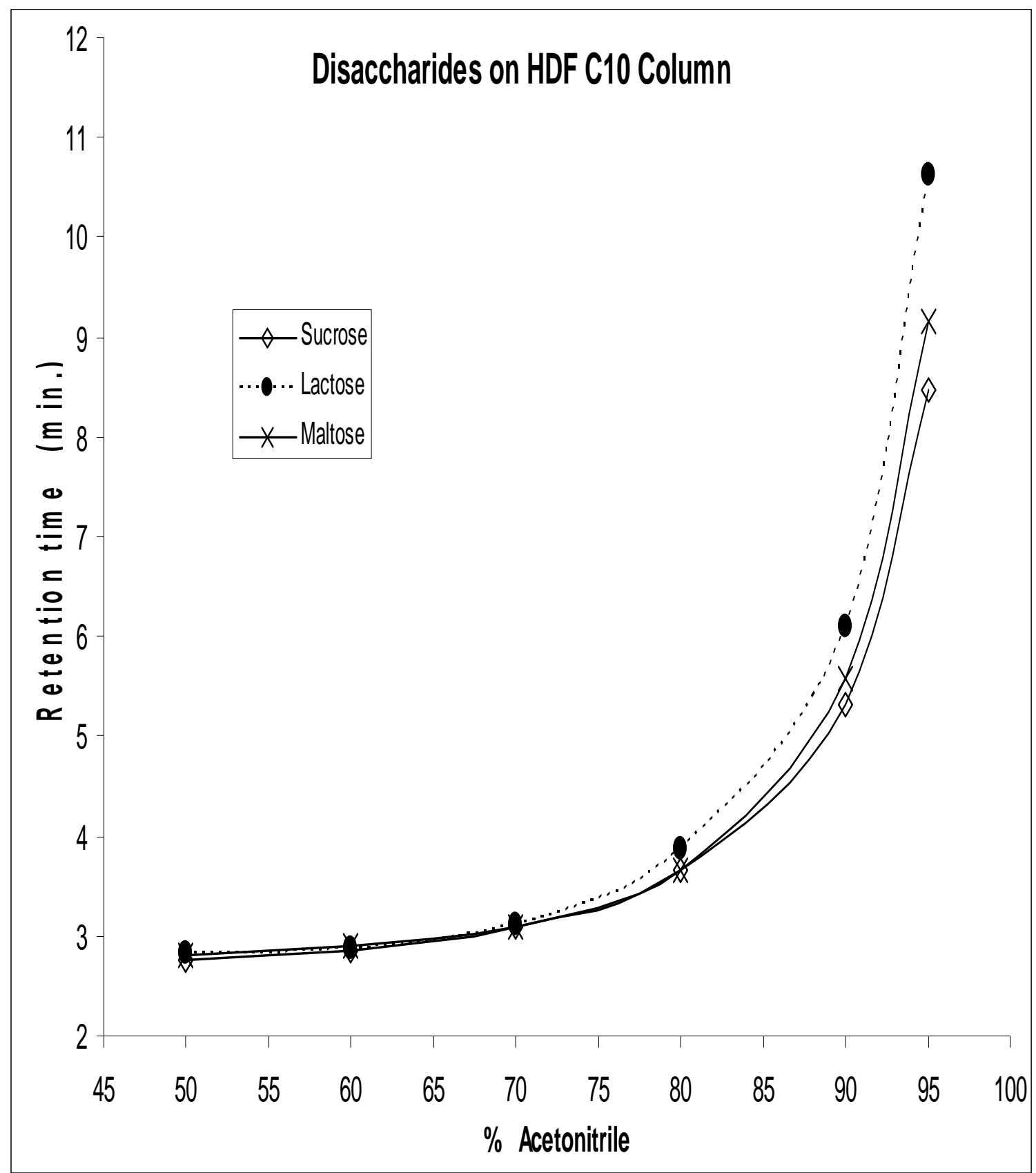

Figure 53. Retention map of disaccharides on the HDF C10 column. 


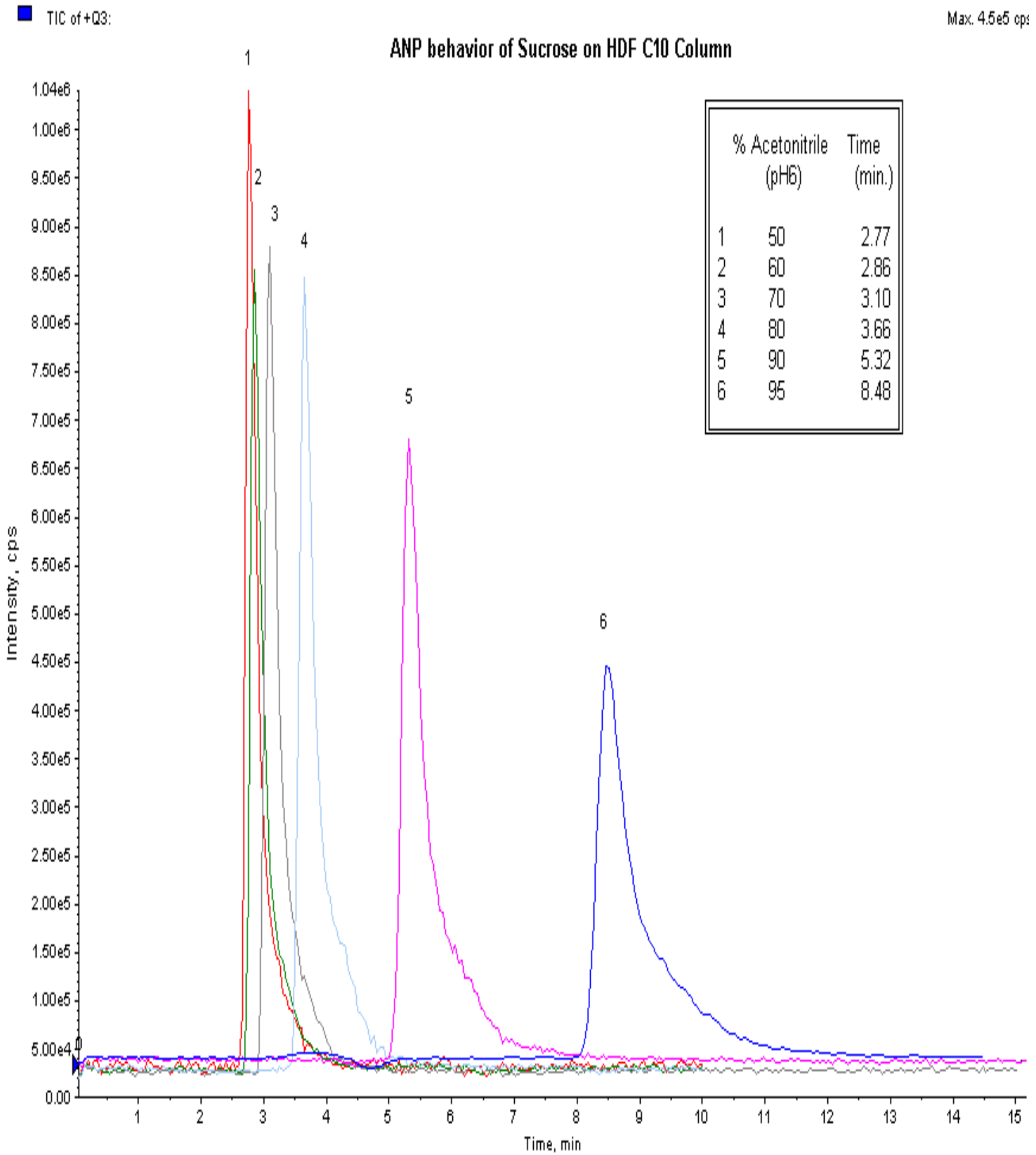

Figure 54. Aqueous normal phase chromatographic retention of sucrose as acetonitrile part of mobile phase increases from $50 \%$ to $95 \%$ on the HDF C10 column. 
Figure 54 shows the chromatographic peak for the ANP retention of sucrose on the HDF C10 column as the acetonitrile concentration increases from $50 \%$ to $95 \%$. As the retention time increases, the peak gets broader and the intensity of the peak decreases with increasing acetonitrile concentration. The observed tailing could be reduced by using a lower concentration sample solutions on the LC-MS system. This chromatographic peak shape could be improved through the use of gradient methods. The use of a gradient can easily overcome the problem of peak tailing due to the slow mass transfer kinetics of the ANP mechanism.

Furthermore, the polar disaccharides trehalose and turanose were analyzed by LCMS in parallel with the nonpolar disaccharide cellobiose octaacetate to study simultaneous ANP and reversed phase behavior of the TDF C8 and HDF C10 columns. Due to the fact that trehalose and turanose also have the exact same molecular weight as sucrose, lactose, and maltose [as shown in Table 19 (C)], the exact same $\mathrm{m} / \mathrm{z}$ of 365.30 amu sodium adduct ions were obtained using the ESI positive ion mode scanning. This $\mathrm{m} / \mathrm{z}$ was scanned to record the SIM chromatogram for trehalose and turanose on both columns. For cellobiose octaacetate which has molecular weight $678.3 \mathrm{amu}$, the $\mathrm{m} / \mathrm{z}$ $701.2 \mathrm{amu}$ of its sodium adduct ion was observed, and this ion was scanned to obtain the SIM chromatogram for the study of reversed phase retention on the TDF C8 and HDF C10 columns.

For trehalose and turanose, a $0.5 \mu \mathrm{L}$ injection volume of a $1 \mathrm{mg} / \mathrm{mL}$ sample solution was used for the retention analysis on the TDF C8 and HDF C10 columns. For dissolving the samples of trehalose and turanose to $1 \mathrm{mg} / \mathrm{mL}$, a 50:50 acetonitrile:water 
at $\mathrm{pH} \sim 6$ was used. The cellobiose octaacetate was undissolved in 50:50

acetonitrile:water at $\mathrm{pH} \sim 6$. After several trials with different mobile phase compositions, a 90:10 acetonitrile:water with $\mathrm{pH} \sim 6$ was used to dissolve the cellobiose octaacetate to make the $1 \mathrm{mg} / \mathrm{mL}$ sample solution. A $0.5 \mu \mathrm{L}$ injection volume was used which resulted in very sharp peaks.

Table 26 presents the retention time data of trehalose, turanose and cellobiose octa acetate analyzed on the TDF C8 column. It is a very interesting set of retention data obtained using an isocratic run under same experimental conditions on the same column. Both trehalose and turanose being polar disaccharides, start retaining from 50:50 acetonitrile:water $\mathrm{pH} \sim 6$ mobile phase condition up to a very high ANP retention of 10.66 minutes and 9.25 minutes respectively for $95 \%$ acetonitrile. However, cellobiose octaacetate is a nonpolar disaccharide which shows a decrease in retention as the concentration of the acetonitrile increases. When cellobiose octaacetate was run using 20:80 acetonitrile:water $\mathrm{pH} \sim 6$ reversed phase mobile phase condition, it showed a significant retention of 10.33 minutes on the TDF C8 column. These analyses successfully elucidate that the TDF C8 column can retain both polar as well nonpolar saccharides showing ANP and reversed phase behavior.

Figure 55 shows the SIM chromatographic peak shape for the ANP retention of turanose at 50:50, 60:40, 70:30, 80:20, 95:05 acetonitrile:water $\mathrm{pH} \sim 6$ mobile phase compositions. Figure 56 and Figure 57 are the individual ANP retention maps of trehalose and turanose respectively. All the peaks obtained in the retention study of 
trehalose, turanose and cellobiose octaacetate were highly symmetric and sharp with very good signal to noise ratio.

Table 26. Retention times of polar and nonpolar disaccharides on the TDF C8 column using LCMS

\begin{tabular}{|c|c|c|c|c|}
\hline \multicolumn{2}{|c|}{$\begin{array}{c}\text { Concentration } \\
(\%)\end{array}$} & \multicolumn{3}{|c|}{$\begin{array}{l}\text { Retention time } \\
\text { (min) }\end{array}$} \\
\hline $\begin{array}{c}\text { Acetonitrile } \\
+ \\
(15 \mathrm{mM} \\
\text { Ammonium- } \\
\text { formate })\end{array}$ & $\begin{array}{c}\text { DI Water } \\
+ \\
(15 \mathrm{mM} \\
\text { Ammonim- } \\
\text { formate })\end{array}$ & $\begin{array}{c}\text { Trehalose } \\
\text { anhydrous }\end{array}$ & Turanose & $\begin{array}{l}\text { Cellobiose } \\
\text { octaacetate } \\
\text { (nonpolar) }\end{array}$ \\
\hline 50 & 50 & 2.87 & 2.95 & 3.61 \\
\hline 60 & 40 & 2.97 & 2.96 & 3.24 \\
\hline 70 & 30 & 3.22 & 3.17 & 2.96 \\
\hline 80 & 20 & 3.86 & 3.83 & 2.78 \\
\hline 90 & 10 & 6.07 & 5.71 & 2.76 \\
\hline 95 & 05 & 10.66 & 9.25 & 2.76 \\
\hline 20 & 80 & - & - & 10.30 \\
\hline
\end{tabular}




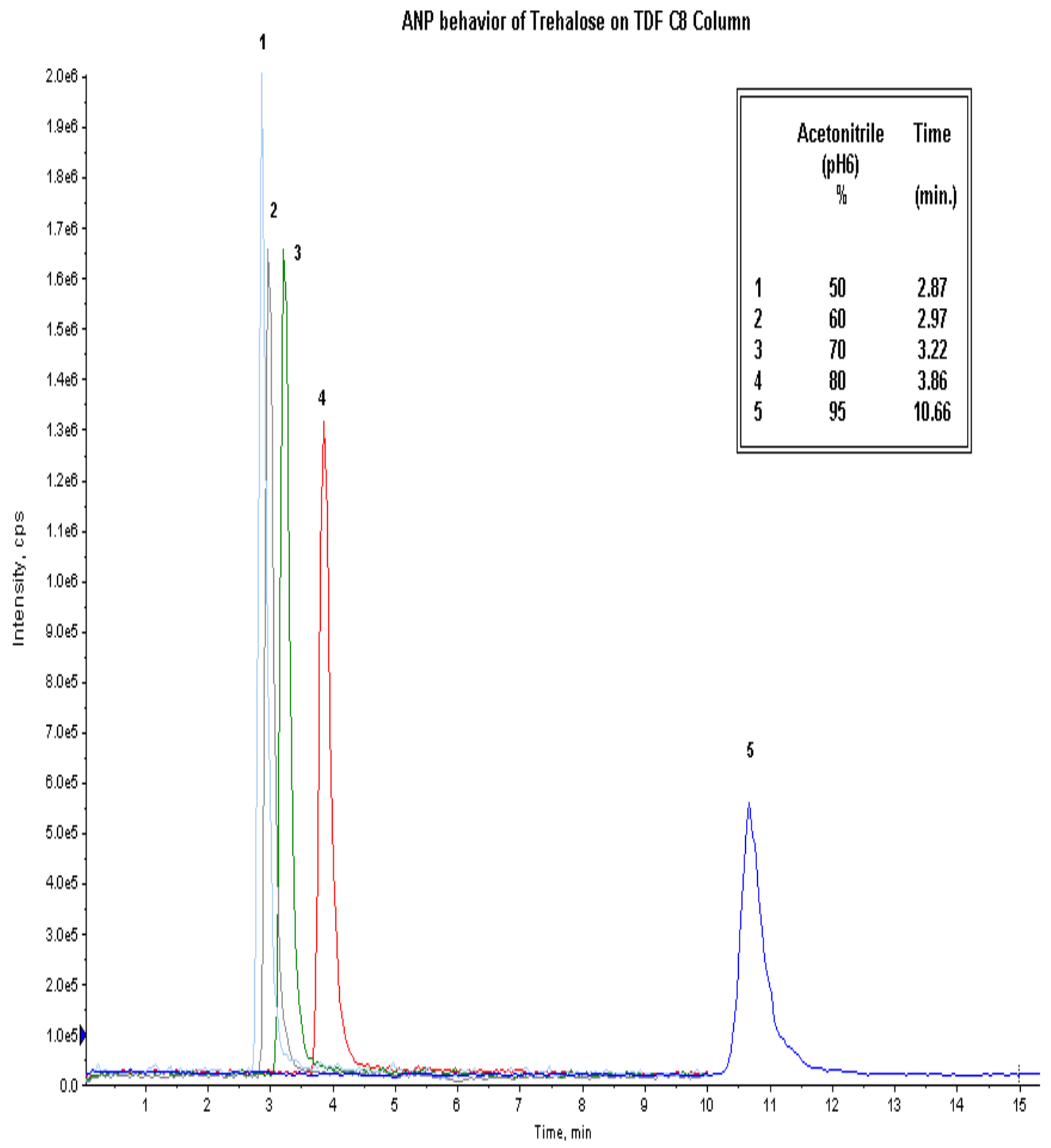

Figure 55. Aqueous normal phase chromatographic retention of trehalose as acetonitrile part of mobile phase increases from $50 \%$ to $95 \%$ on the TDF C8 column. 


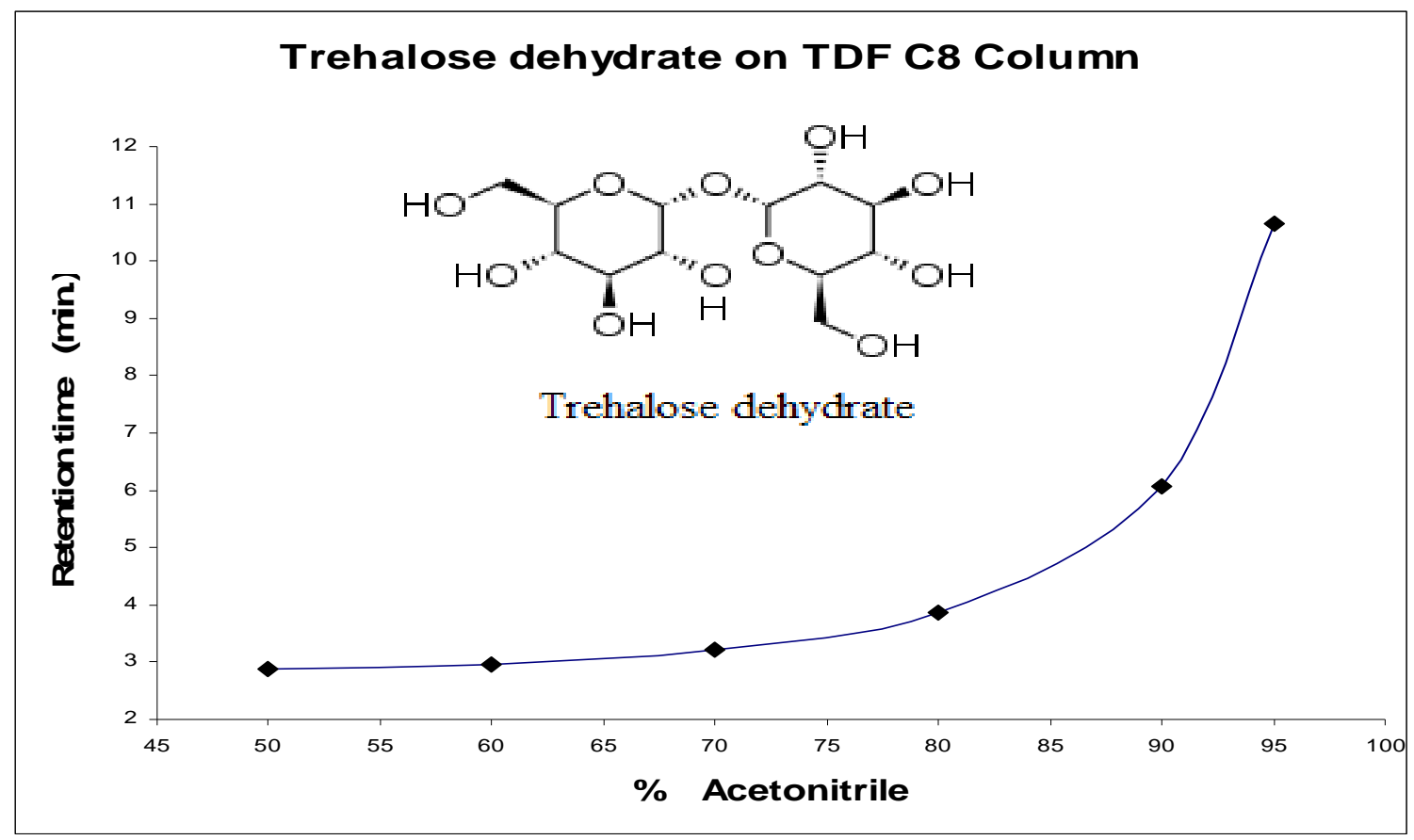

Figure 56. ANP retention of trehalose dehydrate on the TDF C8 column.

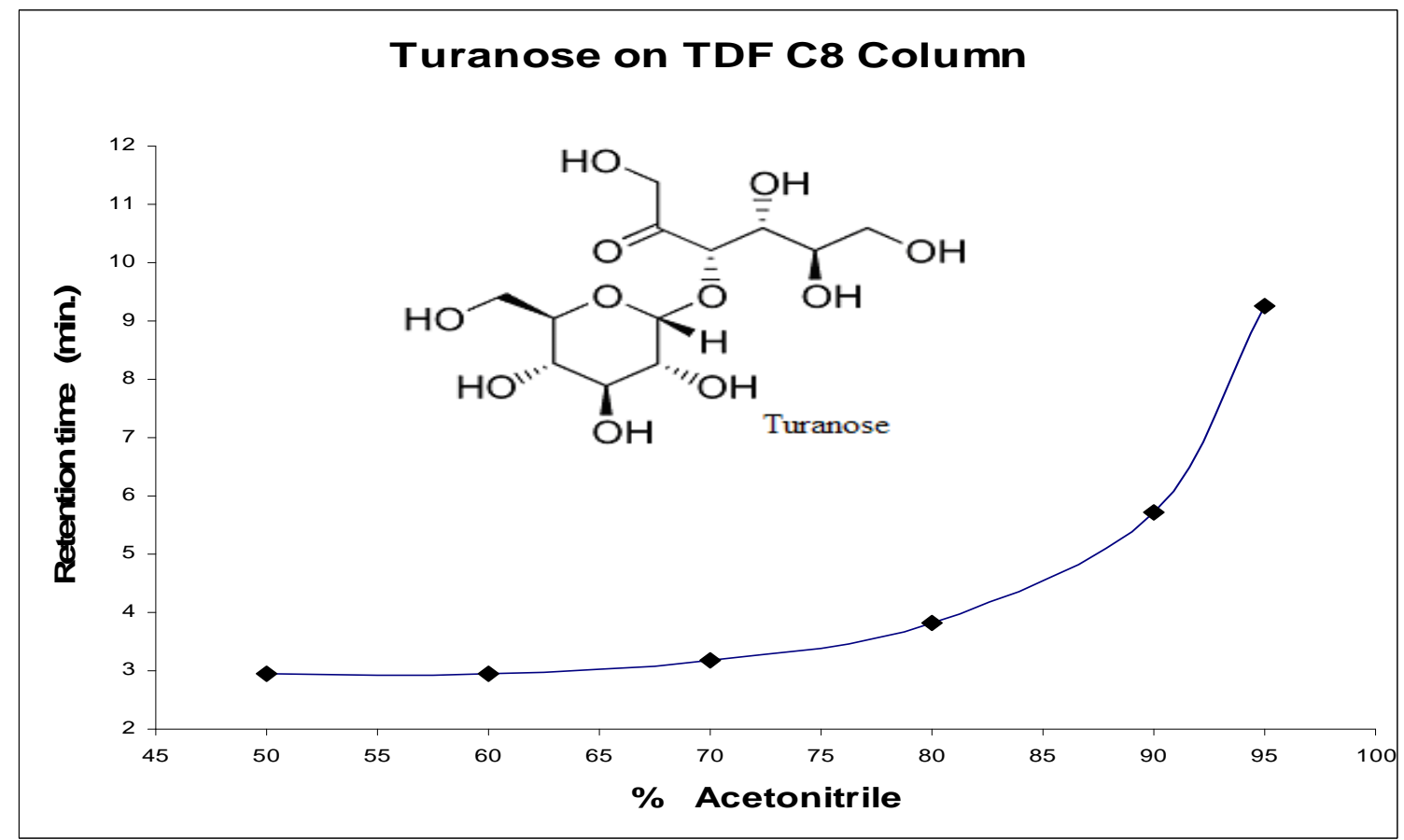

Figure 57. ANP retention of turanose on the TDF C8 column. 
TIC of +Q3: from Sample 2 (CEL-2) of \#1CELPos:01 center5050.wiff

Max. 6.1e6 cps.

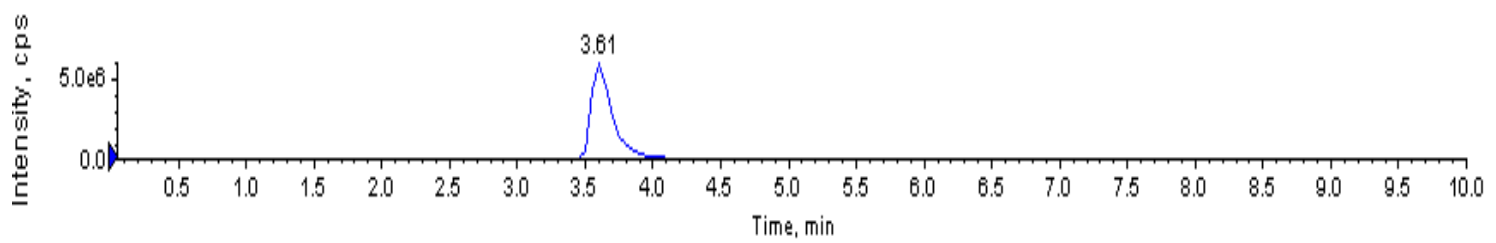

TIC of +03: from Sample 1(CEL) of \#1CELpos0101 centerf040.wiff

Max. $1.0 \mathrm{e} 7 \mathrm{cps}$

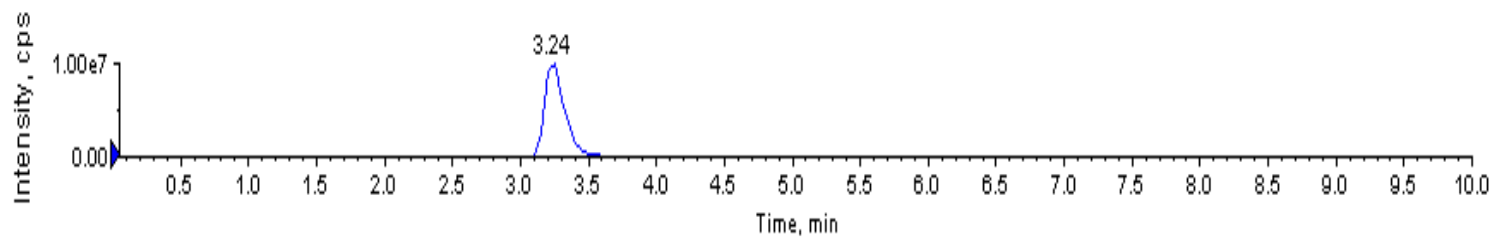

TIC of +03: from Sample 1 (CEL-1) of \#1CELPos0101 center8020.wiff

Max. $1.5 \mathrm{e} 7 \mathrm{cps}$

告

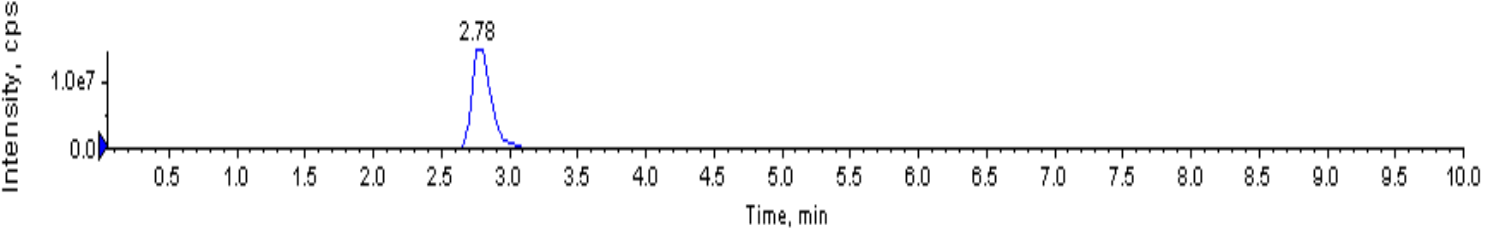

TIC of +03: from Sample 1 (CEL-1) of \#1CELPos0101 center8010.wiff

Max.7.5e6 cps

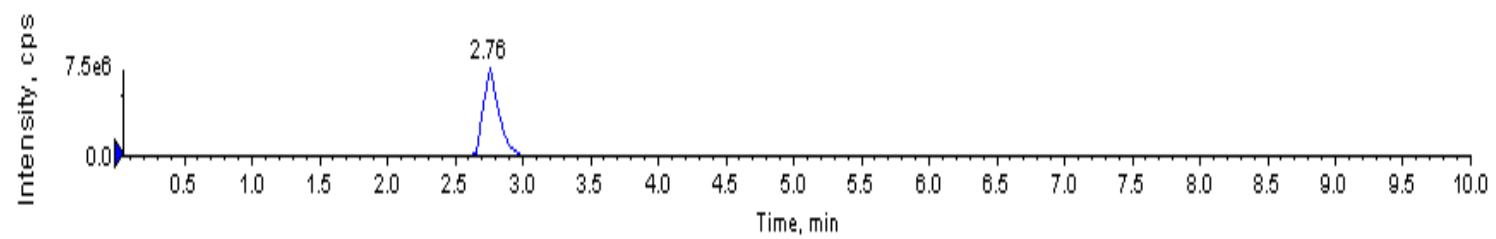

TIC of +03: from Sample 1 (CEL-1) of \#1CELPOS001 center2080.wiff

Max. $3.5 .5 \mathrm{cps}$

0
$\frac{0}{0}$
0
$\vdots$
0
0
0
0
0

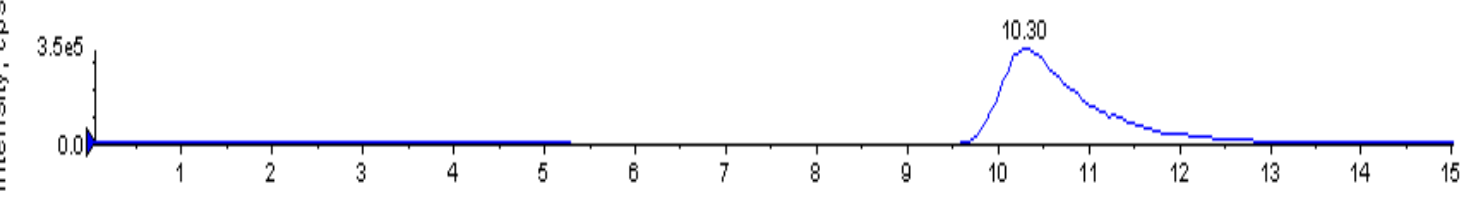

Figure 58. Reversed phase retention of nonpolar cellobiose octaacetate on the TDF C8 column in the form of SIM of $(\mathrm{M}+\mathrm{Na})^{+}$adduct $=701.2 \mathrm{amu}$ using ESI (+) mode MS analysis. 
The first four panels of Figure 58 show the SIM chromatograms demonstrating the $\mathrm{RP}$ retention time of cellobiose octaacetate for 50:50, 60:40, 80:20, and 90:10 acetonitrile:water $\mathrm{pH} \sim 6$ mobile phase compositions on the TDF C8 column. The last panel represents the SIM chromatogram of the reversed phase retained chromatographic peak of cellobiose octaacetate at 20:80 acetonitrile:water $\mathrm{pH} \sim 6$ mobile phase condition. As the concentration of acetonitrile increases from $50 \%$ to $90 \%$, the retention time decreases from 3.61 minutes to 2.76 minutes and it elutes faster with the higher amount of nonpolar acetonitrile which is evidence of the RP behavior of the column. At the composition of 20:80 acetonitrile:water $\mathrm{pH} \sim 6$ mobile phase condition, the higher amount of water in the mobile phase system retains the nonpolar solute cellobiose octaacetate on the TDF C 8 column for 10.66 minutes. This retention is further evidence for the reversed phase retention of this compound.

The exact same reversed phase retention behavior was also observed for the nonpolar cellobiose octaacetate on the HDF C10 column under similar experimental conditions. Table 27 lists the retention times for polar trehalose and turanose disaccharides along with the nonpolar cellobiose octaacetate disaccharide analyzed on HDF C10 column using similar mobile phase conditions. On the HDF C10 column, cellobiose octaacetate was retained up to 7.49 minutes using reversed phase conditions. The higher reversed phase retention of this nonpolar solute observed on the TDF C8 column (10.66 minutes) than the HDF C10 column (7.49 minutes) is due to the fact that the TDF C8 has a higher surface coverage than the HDF C10. 
Table 27. Retention times of polar and nonpolar disaccharides on the HDF C10 column using LCMS

\begin{tabular}{|c|c|c|c|c|}
\hline \multicolumn{2}{|c|}{$\begin{array}{c}\text { Concentration } \\
(\%)\end{array}$} & \multicolumn{3}{|c|}{$\begin{array}{l}\text { Retention time } \\
\text { (min) }\end{array}$} \\
\hline $\begin{array}{c}\text { Acetonitrile } \\
+ \\
(15 \mathrm{mM} \\
\text { Ammonium- } \\
\text { formate })\end{array}$ & $\begin{array}{c}\text { DI Water } \\
+ \\
(15 \mathrm{mM} \\
\text { Ammonim- } \\
\text { formate })\end{array}$ & $\begin{array}{l}\text { Trehalose } \\
\text { anhydrous }\end{array}$ & Turanose & $\begin{array}{c}\text { Cellobiose } \\
\text { octaacetate } \\
\text { (nonpolar) }\end{array}$ \\
\hline 50 & 50 & 2.76 & 2.85 & 3.53 \\
\hline 60 & 40 & 2.82 & 2.91 & 3.12 \\
\hline 70 & 30 & 3.12 & 3.13 & 2.81 \\
\hline 80 & 20 & 3.80 & 3.77 & 2.71 \\
\hline 90 & 10 & 6.10 & 5.67 & 2.66 \\
\hline 95 & 05 & 10.74 & 9.35 & 2.65 \\
\hline 20 & 80 & - & - & 7.49 \\
\hline
\end{tabular}




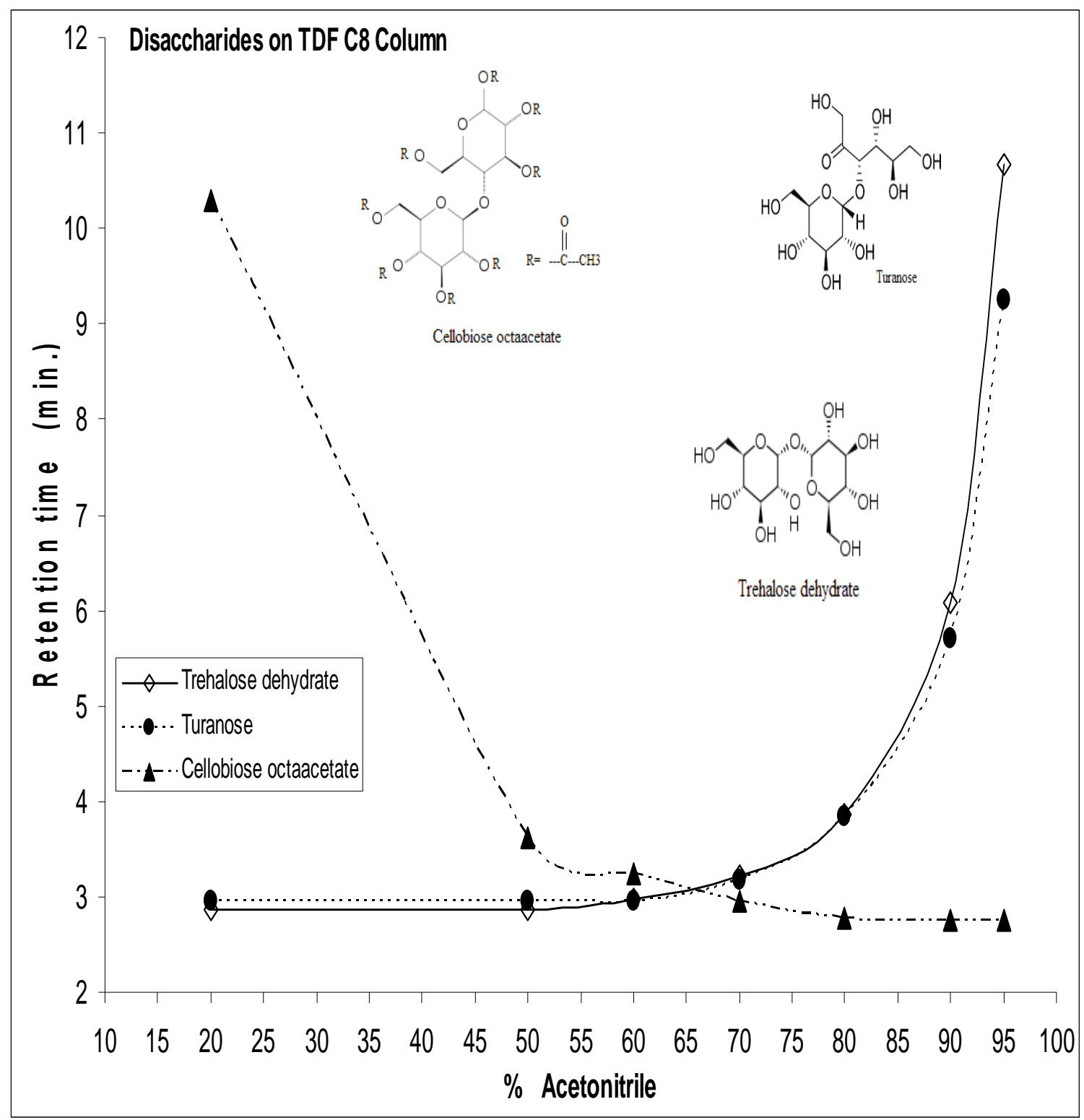

Figure 59. Retention map of polar and nonpolar disaccharides on the TDF C8 column using isocratic run. 


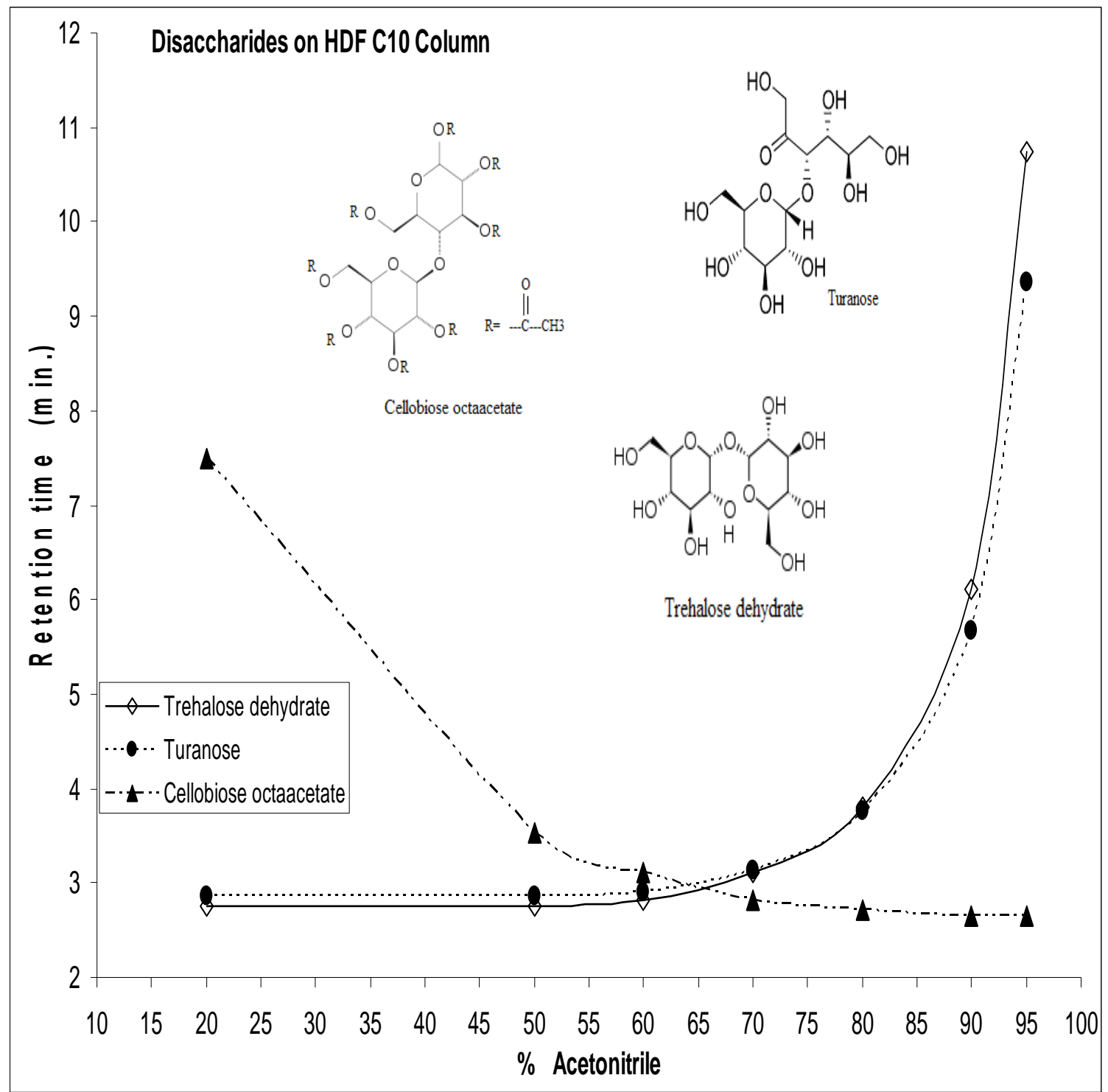

Figure 60. Retention map of polar and nonpolar disaccharides on the HDF C10 column using isocratic run.

Figure 59 and Figure 60 represent the ANP retention maps of polar trehalose and turanose along with the reversed phase retention map of nonpolar cellobiose octaacetate for the TDF C8 column and the HDF C10 column respectively with varying acetonitrile 
concentration. As already pointed out earlier in the ANP reference literature, both of these plots indicate points at which polar and nonpolar solutes could be expected to be retained if that particular mobile phase condition is tried. This ability of the silica hydride based columns to be able to have both aqueous normal phase and reversed phase behavior can be very useful for the retention of multipolarity biomolecules.

\subsection{Amino Acids}

The amino acids were analyzed on LC-MS for the purpose of validating the retention data obtained on ELSD detector. The same amino acids, arginine, aspartic acid, phenylalanine, and tyrosine, were analyzed on the TDF C8 and HDF C10 columns. The experimental conditions and results obtained are discussed in the following section.

\section{Verification of Retention Data Using Different Detectors}

An interesting comparison of two modes of detection was conducted by overlaying the ANP retention data obtained using different detectors. A set of amino acids analyzed previously using ELSD were analyzed again on both the TDF C8 and HDF C10 columns using the LC-MS system. Similarly, the ANP retention data of fructose obtained on UV were compared with the results obtained using LC-MS for the TDF C8 column. Furthermore, the ANP retention data of trehalose and turanose analyzed previously using UV were compared with the results obtained using LC-MS on the TDF C8 column.

\subsection{Amino Acids on ELSD and LCMS}

The experimental conditions for the amino acids were reported in the section above describing the analysis using the ELSD detector. For analyzing amino acids using the LC-MS system, a $0.4 \mathrm{~mL} / \mathrm{min}$ flow rate was used. For each amino acid, the sample 
solution of $1 \mathrm{mg} / \mathrm{mL}$ was prepared using 50:50 acetonitarile:water premixed with $0.1 \%$ FA. The injection volume for arginine, aspartic acid, phenylalanine, and tyrosine was 0.5 $\mu \mathrm{L}$ on both columns. The $[\mathrm{M}+1]^{+1}$ molecular ions of the amino acids were recorded using the Q3 MS positive polarity scan mode. As presented in Table 19 (B), the $\mathrm{m} / \mathrm{z}$ values of the $[\mathrm{M}+1]^{+1}$ molecular ions of arginine, aspartic acid, phenylalanine, and tyrosine were $175.20 \mathrm{amu}, 134.10 \mathrm{amu}, 166.19 \mathrm{amu}$, and $182.19 \mathrm{amu}$ respectively. For the molecular ions to record the SIM chromatograms, the SIM window width was kept at $0.5 \mathrm{amu}$ for all the amino acids except for the arginine for which the width was kept at $0.3 \mathrm{amu}$ in order to receive sharp peaks.

Table 28 and Table 29 list the retention times of these amino acids observed on the TDF C8 and the HDF C10 columns for the mobile phase compositions of 50:50, 60:40, 70:30, and 80:20 acetonitrile:water premixed with $0.1 \% \mathrm{FA}$. The strong basic characteristic of arginine results in very high ANP retention as the concentration of acetonitirle increases from $50 \%$ to $70 \%$. To accommodate the ANP retention time data obtained using ELSD and LC-MS on the same plot, the retention times of only aspartic acid, phenylalanine, and tyrosine were plotted as shown in Figure 63 and Figure 64 for the TDF C8 and HDF C10 columns respectively. The results for arginine were not plotted due to its very high ANP retention at a $0.4 \mathrm{~mL} / \mathrm{min}$ flow rate.

The retention maps of the amino acids analyzed using ELSD and LC-MS are comparable. Although the flow rates and other experimental factors might be different for each analysis, the trend of ANP retention for phenylalanine, aspartic acid and tyrosine 
on both columns are comparable. Hence, the readings taken on LC-MS validate all the ELSD data recorded for both columns.

Table 28. Retention times of amino acids on the TDF C8 column using LCMS

\begin{tabular}{|c|c|c|c|c|c|}
\hline \multicolumn{2}{|c|}{$\begin{array}{c}\text { Concentration } \\
\text { (\%) }\end{array}$} & \multicolumn{4}{|c|}{$\begin{array}{c}\begin{array}{c}\text { Retention time } \\
(\mathrm{min})\end{array} \\
\text { (for } 0.4 \mathrm{~mL} / \mathrm{min} \text { flow rate) }\end{array}$} \\
\hline $\begin{array}{c}\text { Acetonitrile } \\
\text { with } \\
0.1 \% \mathrm{FA}\end{array}$ & $\begin{array}{c}\text { DI water } \\
\text { with } \\
0.1 \% \mathrm{FA}\end{array}$ & PHE & ASP & TYR & $\mathrm{ARG}$ \\
\hline 50 & 50 & 4.57 & 3.63 & 4.31 & 8.47 \\
\hline 60 & 40 & 5.22 & 4.16 & 5.11 & 12.68 \\
\hline 70 & 30 & 6.46 & 5.35 & 6.42 & 24.90 \\
\hline 80 & 20 & 9.83 & 8.21 & 10.52 & - \\
\hline 85 & 15 & 13.73 & 12.37 & 13.77 & - \\
\hline
\end{tabular}


Table 29. Retention times of amino acids on the HDF C10 column using LCMS

\begin{tabular}{|c|c|c|c|c|c|}
\hline \multicolumn{2}{|c|}{$\begin{array}{c}\text { Concentration } \\
\text { (\%) }\end{array}$} & \multicolumn{4}{|c|}{$\begin{array}{c}\begin{array}{c}\text { Retention time } \\
(\mathrm{min})\end{array} \\
\text { (for } 0.4 \mathrm{~mL} / \mathrm{min} \text { flow rate) }\end{array}$} \\
\hline $\begin{array}{c}\text { Acetonitrile } \\
\text { with } \\
0.1 \% \mathrm{FA}\end{array}$ & $\begin{array}{c}\text { DI water } \\
\text { with } \\
0.1 \% \mathrm{FA}\end{array}$ & PHE & ASP & TYR & $\mathrm{ARG}$ \\
\hline 50 & 50 & 4.46 & 3.56 & 4.21 & 8.32 \\
\hline 60 & 40 & 5.06 & 4.10 & 4.88 & 12.81 \\
\hline 70 & 30 & 6.31 & 5.21 & 6.45 & 25.08 \\
\hline 80 & 20 & 9.75 & 8.32 & 9.44 & - \\
\hline 85 & 15 & 13.21 & 11.77 & 14.28 & - \\
\hline
\end{tabular}




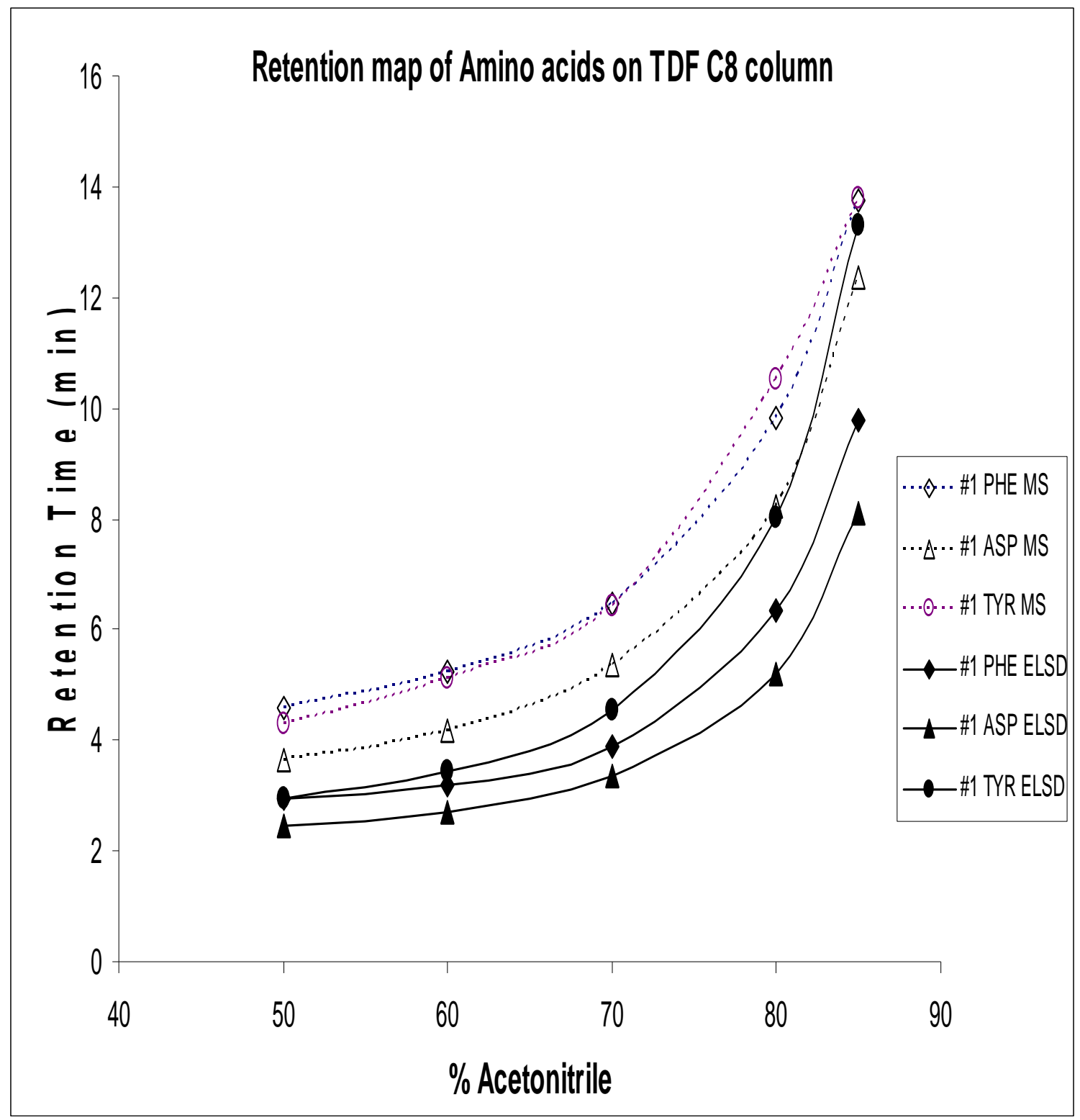

Figure 61. Verification of the retention maps of amino acids on the TDF C8 column using ELSD and MS detectors: $0.4 \mathrm{~mL} / \mathrm{min}$ flow rate was used in HPLC-MS analysis and $0.5 \mathrm{~mL} / \mathrm{min}$ flow rate was used in HPLC/ELSD analysis. 


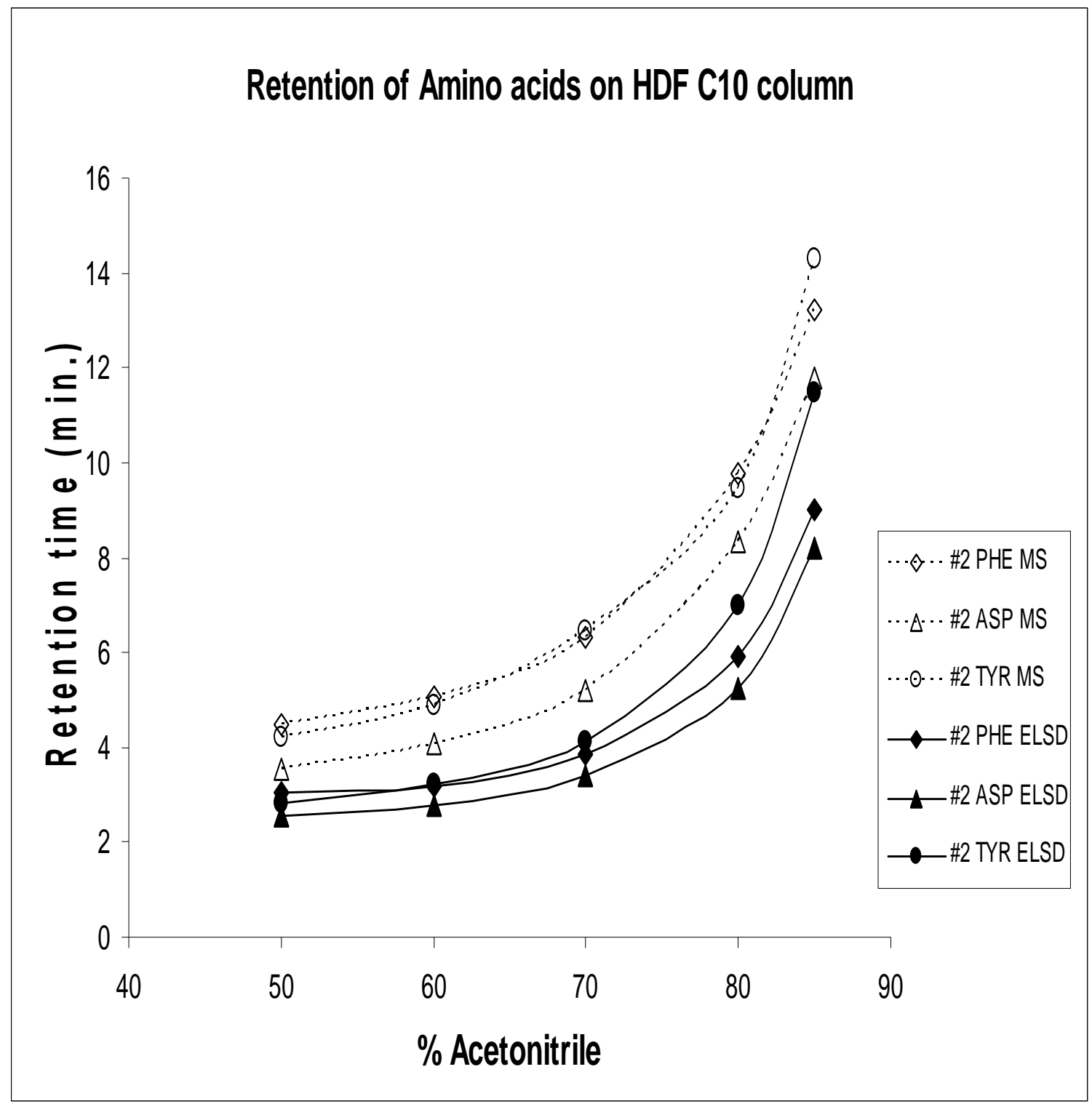

Figure 62. Verification of the retention maps of amino acids on the HDF C10 column using ELSD and MS detectors: $0.4 \mathrm{~mL} / \mathrm{min}$ flow rate was used in HPLC-MS analysis and $0.5 \mathrm{~mL} / \mathrm{min}$ flow rate was used in HPLC/ELSD analysis. 


\subsection{Monosaccharide and disaccharides on UV and LC-MS}

The ANP retention data of D-fructose analyzed by UV using a $0.3 \mathrm{~mL} / \mathrm{min}$ flow rate are plotted with the data analyzed on LC-MS using a $0.4 \mathrm{~mL} / \mathrm{min}$ flow rate. Table 30 gives the retention times of fructose analyzed on the TDF C8 column using both UV and LC-MS modes of detection. Figure 69 shows the ANP retention maps obtained using UV and LC-MS detectors. Although the retention times are different along with the other experimental conditions, the shapes of the retention maps obtained on both the detectors are comparable to validate the retention data of fructose obtained on UV.

Table 30. Retention times of monosaccharide on the TDF C8 column using LCMS and UV

\begin{tabular}{|c|c|c|c|}
\hline \multicolumn{2}{|c|}{$\begin{array}{c}\text { Concentration } \\
\text { (\%) }\end{array}$} & \multicolumn{2}{c|}{$\begin{array}{c}\text { Retention time of Fructose } \\
\text { (min) }\end{array}$} \\
\hline $\begin{array}{c}\text { Acetonitrile } \\
(15 \mathrm{mM} \\
\begin{array}{c}\text { Ammonium } \\
\text { formate })\end{array}\end{array}$ & $\begin{array}{c}\text { Ammonium } \\
\text { formate })\end{array}$ & LCMS & $(0.3 \mathrm{~mL} / \mathrm{min})$ \\
\hline 50 & 50 & 2.92 & 4.01 \\
\hline 60 & 40 & 3.00 & 3.98 \\
\hline 70 & 30 & 3.20 & 4.40 \\
\hline 80 & 20 & 3.65 & 5.21 \\
\hline 90 & 10 & 4.70 & 7.88 \\
\hline
\end{tabular}




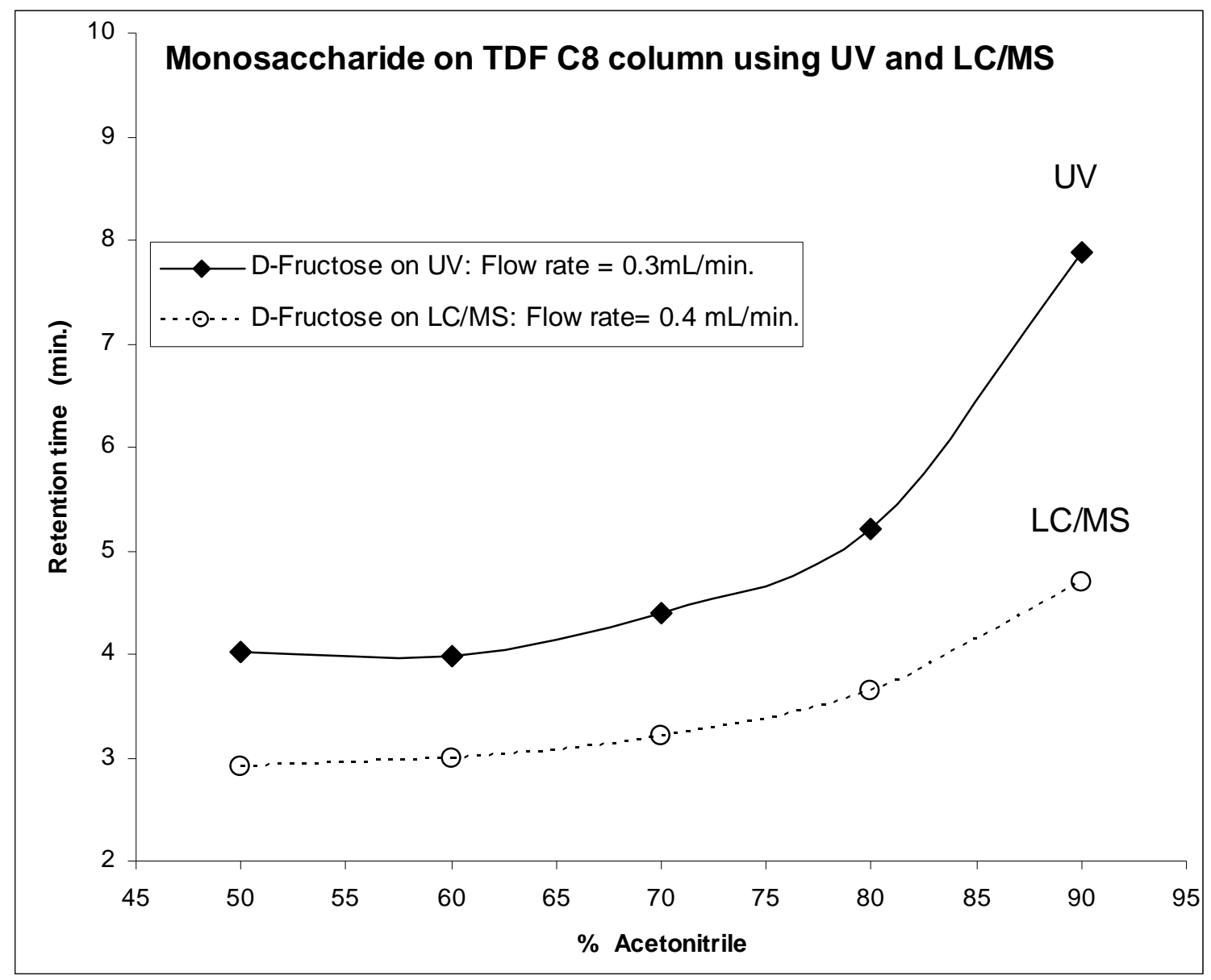

Figure 63. Verifying ANP retention of monosaccharide on UV by LCMS.

The disaccharides turanose and trehalose analyzed on the TDF C8 column using UV were also used for a verification study. The experimental conditions for UV analysis are as follows. The flow rate was set to $0.5 \mathrm{~mL} / \mathrm{min}$, and the UV absorption wavelength was $220 \mathrm{~nm}$. The sample solution at a concentration of $3 \mathrm{mg} / \mathrm{mL}$ was made using 50:50 acetonitrile:water $\mathrm{pH} \sim 6$. An injection volume of $20 \mu \mathrm{L}$ was used. The negative peaks were measured to record the ANP retention times and they are tabulated in Table 31 along with the comparable retention time data obtained using LC-MS. Figure 62 shows 
the retention maps of turanose and trehalose demonstrating the comparable shapes analyzed on UV and LC-MS. These similar shaped retention maps validate the retention data analyzed on UV.

Table 31. Retention times of disaccharides on the TDF C8 column using LCMS and UV

\begin{tabular}{|c|c|c|c|c|c|}
\hline \multicolumn{2}{|c|}{$\begin{array}{c}\text { Concentration } \\
(\%)\end{array}$} & \multicolumn{4}{|c|}{$\begin{array}{l}\text { Retention time } \\
\qquad \text { (min) }\end{array}$} \\
\hline \multirow{2}{*}{$\begin{array}{c}\text { Acetonitrile } \\
\text { (15mM Ammonium } \\
\text { formate) }\end{array}$} & \multirow{2}{*}{$\begin{array}{l}\text { DI Water } \\
\text { (15mM } \\
\text { Ammonium } \\
\text { formate })\end{array}$} & \multicolumn{2}{|c|}{$\begin{array}{c}\text { LCMS } \\
(0.4 \mathrm{~mL} / \mathrm{min})\end{array}$} & \multicolumn{2}{|c|}{$\begin{array}{c}\mathbf{U V} \\
(0.5 \mathrm{~mL} / \mathrm{min})\end{array}$} \\
\hline & & Turanose & Trehalose & Turanose & Trehalose \\
\hline 50 & 50 & 2.95 & 2.87 & 2.23 & 2.21 \\
\hline 60 & 40 & 2.96 & 2.97 & 2.32 & 2.33 \\
\hline 70 & 30 & 3.17 & 3.22 & 2.54 & 2.54 \\
\hline 80 & 20 & 3.83 & 3.86 & 3.15 & 3.15 \\
\hline 90 & 10 & 5.71 & 6.07 & 5.24 & 5.29 \\
\hline
\end{tabular}




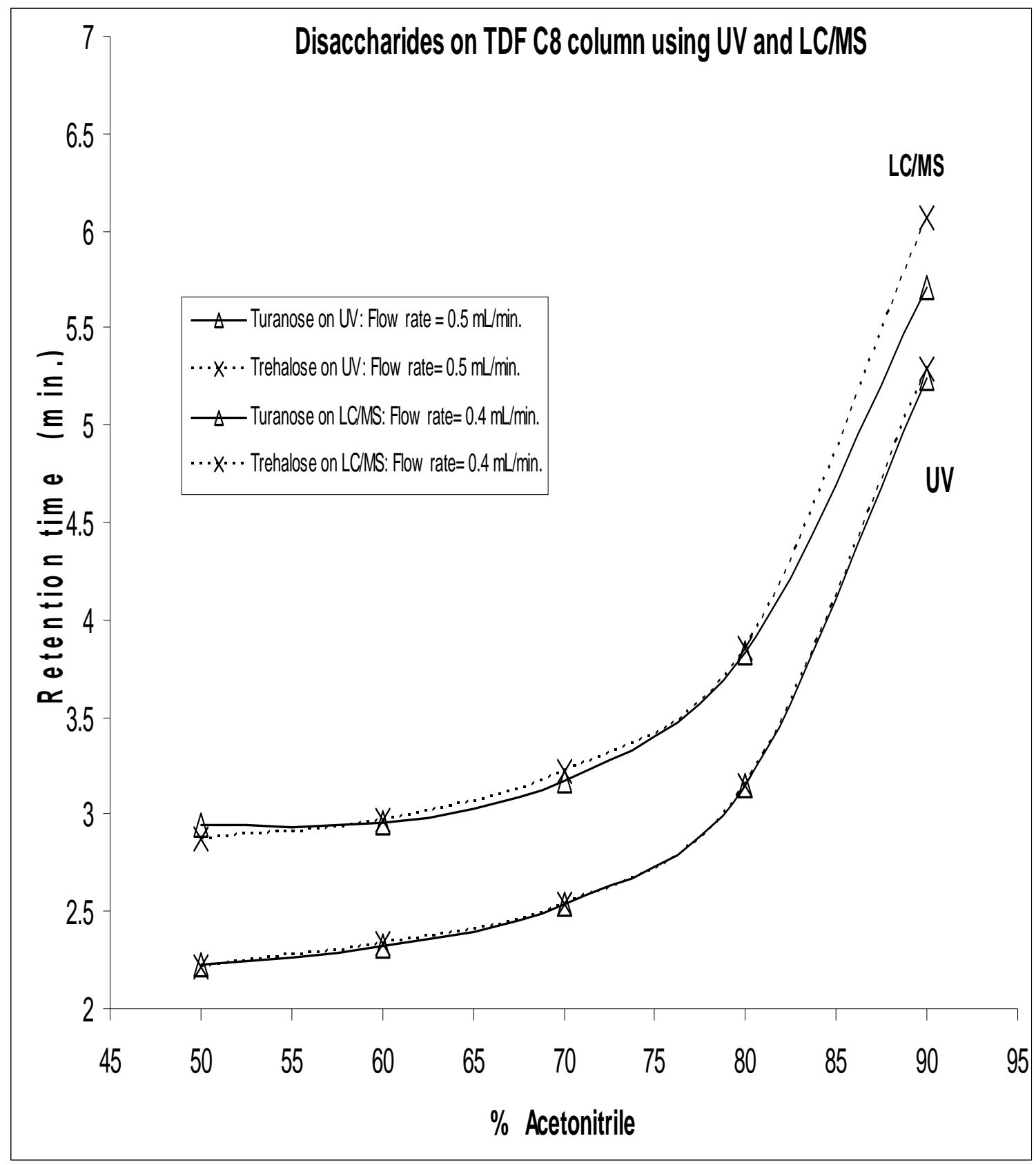

Figure 64. Verifying ANP retention of disaccharides on UV by LCMS. 


\section{Acetone Based ANP Retention}

An effort was made to analyze some well retained polar solutes using acetone as the nonpolar part of the ANP binary solvent system. Since acetonitrile can be difficult to handle, difficult to dispose, and expensive to get (due to global shortage of acetonitrile observed in 2009), establishing ANP retention using acetone would be worthwhile.

Fumaric acid and citric acid were analyzed on the TDF C8 column using $15 \mathrm{mM}$ ammonium formate. A $2 \mathrm{mg} / \mathrm{mL}$ sample solution was made by dissolving these organic acids into 50:50 acetone:water at $\mathrm{pH} \sim 7.20$. To achieve a $\mathrm{pH}$ in the range of 6 to 7 , a 15 $\mathrm{mM}$ ammonium formate was dissolved in $10 \%$ of water and then acetone was added to make the desired volume. $\mathrm{A} \mathrm{pH} \sim 7$ water mobile phase was made by dissolving ammonium formate to get a concentration of $15 \mathrm{mM}$. An injection volume of $2.0 \mu \mathrm{L}$ was used for the analysis on the TDF C8 column. SIM peaks were monitored using a threshold value of 50, and the SIM window width was kept to $0.5 \mathrm{amu}$. In the case of fumaric acid and citric acid, m/z 114.95 amu and 190.9 amu respectively for the $[\mathrm{M}-1]^{-1}$ molecular ion were selected using ESI negative polarity scanning. Both had good peaks with a high signal to noise ratio. The appearance of some tailing as the acetone concentration increases can be overcome by developing gradient methods. In the case of citric acid, minor peak splitting was apparent for 90:10 acetone:water $\mathrm{pH} \sim 7$ mobile phase condition. In the case of peak splitting, the use of a lower injection volume for that particular mobile phase composition helped achieve single symmetric peak.

At the same time, the nonpolar cellobiose octaacetate was also analyzed using acetone as part of the ANP solvent system on the TDF C8 column. A $1 \mathrm{mg} / \mathrm{mL}$ sample 
solution was made in 90:10 acetone:water at $\mathrm{pH} \sim 7.0$ and a $2.0 \mu \mathrm{L}$ injection volume was used with a threshold value set to 50 . A m/z value of 701.0 amu for the $[\mathrm{M}+\mathrm{Na}]^{+1}$ sodium adduct was monitored for SIM chromatograms using different acetone-water mobile phase conditions.

Table 32 lists the retention time data for fumaric acid, citric acid, and nonpolar cellobiose octaacetate for 20:80, 50:50, 60:40, 70:30, 80:20, 85:15, 90:10, 95:05 acetone:water at $\mathrm{pH} \sim 7$ mobile phase compositions on the TDF C8 column. The increase in concentration from $50 \%$ to $95 \%$ acetone shows an increase in retention times for organic acids and a decrease in retention times for cellobiose octaacetate which is expected ANP behavior of the TDF C8 column for the polar analytes. Another interesting data is the retention time at 20:80 acetone:water $\mathrm{pH} \sim 7$. For this condition, cellobiose octaacetate has a retention time of 7.33 minutes showing TDF C8's complimentary reversed phase behavior for the same run. Fumaric acid and citric acid show the exact same retention time for $20 \%$ and $50 \%$ acetone concentrations. This proves that like acetonitrile, acetone as well could not retain polar compound up to $50 \%$ concentration and starts retaining from $60 \%$ or greater concentration. Figure 65 shows the ANP retention maps of fumaric acid and citric acid on the TDF C8 column using acetone. The ANP and reversed phase dual retention behavior of the TDF C8 column using acetone can be seen in Figure 66.

Another interesting comparison of acetone- and acetonitrile- based retention of organic acids is presented in Table 33. Figure 67 shows ANP retention maps for fumaric acid and citric acid analyzed using acetonitrile and acetone nonpolar solvents on the TDF 
C8 column. Regardless of differences in the retention time data and other experimental conditions, the retention maps in both the cases of ANP solvents are comparable.

Table 32. Retention times for polar and nonpolar solutes using acetone as a binary organic mobile phase solvent on the TDF C8 column using LCMS

\begin{tabular}{|c|c|c|c|c|}
\hline \multicolumn{2}{|c|}{$\begin{array}{c}\text { Concentration } \\
\text { (\%) }\end{array}$} & \multicolumn{3}{|c|}{$\begin{array}{c}\text { Retention time } \\
\text { (min) }\end{array}$} \\
\hline $\begin{array}{c}\text { Acetone } \\
+ \\
(15 \mathrm{mM} \\
\text { Ammonium- } \\
\text { formate })\end{array}$ & $\begin{array}{c}\text { DI Water } \\
+ \\
(15 \mathrm{mM} \\
\text { Ammonim- } \\
\text { formate })\end{array}$ & Fumaric acid & Citric acid & $\begin{array}{c}\text { Cellobiose } \\
\text { octaaacetate }\end{array}$ \\
\hline 20 & 80 & 2.46 & 2.43 & 7.33 \\
\hline 50 & 50 & 2.46 & 2.43 & 3.64 \\
\hline 60 & 40 & 2.59 & 2.49 & 3.40 \\
\hline 70 & 30 & 2.73 & 2.61 & 3.09 \\
\hline 80 & 20 & 3.00 & 2.92 & 2.96 \\
\hline 85 & 15 & 3.22 & 3.24 & 2.91 \\
\hline 90 & 10 & 4.73 & 6.15 & 2.83 \\
\hline 95 & 05 & 8.07 & 14.44 & 2.80 \\
\hline
\end{tabular}




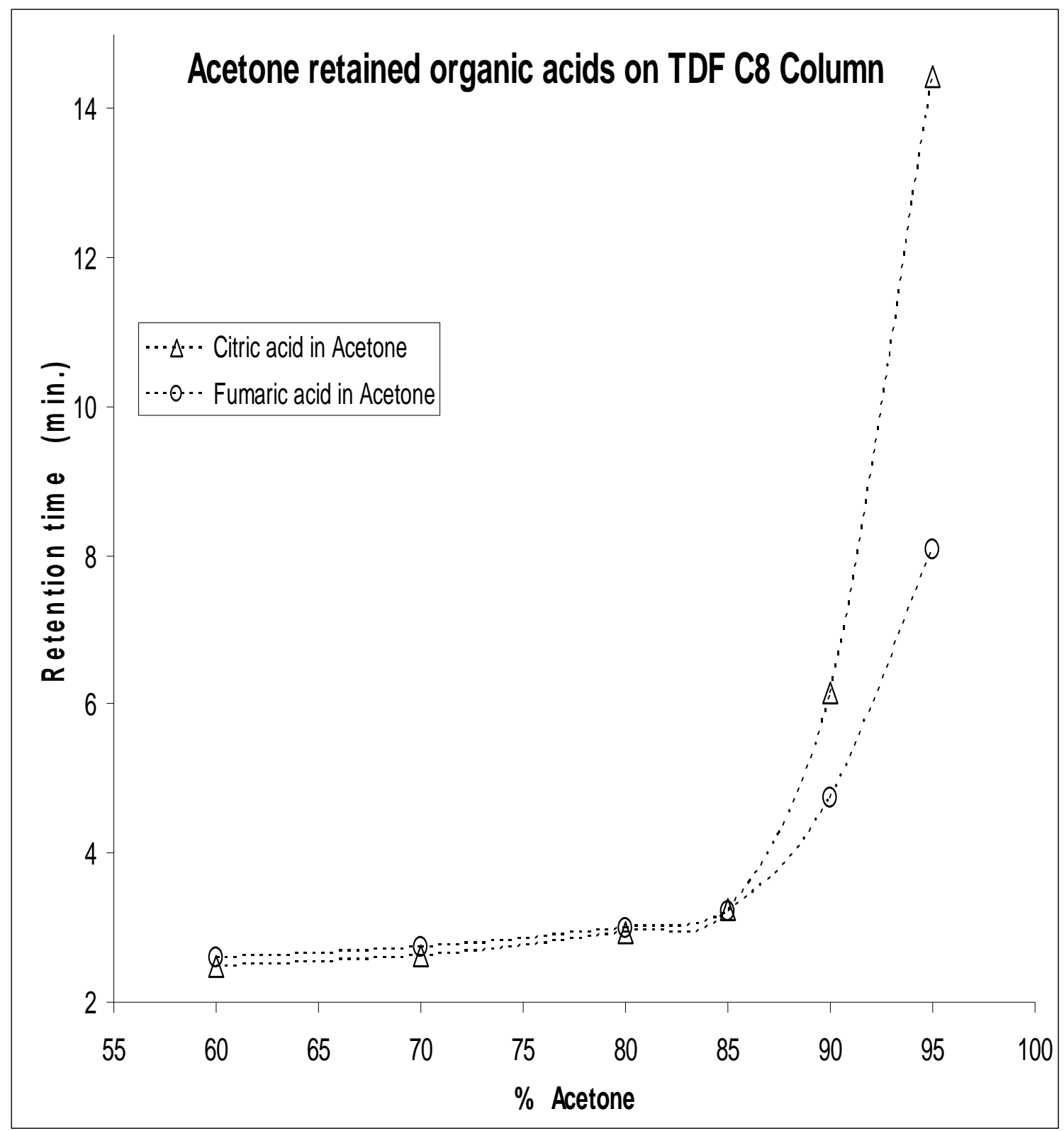

Figure 65. Acetone based ANP retention of organic acids on the TDF C8 column using MS. 


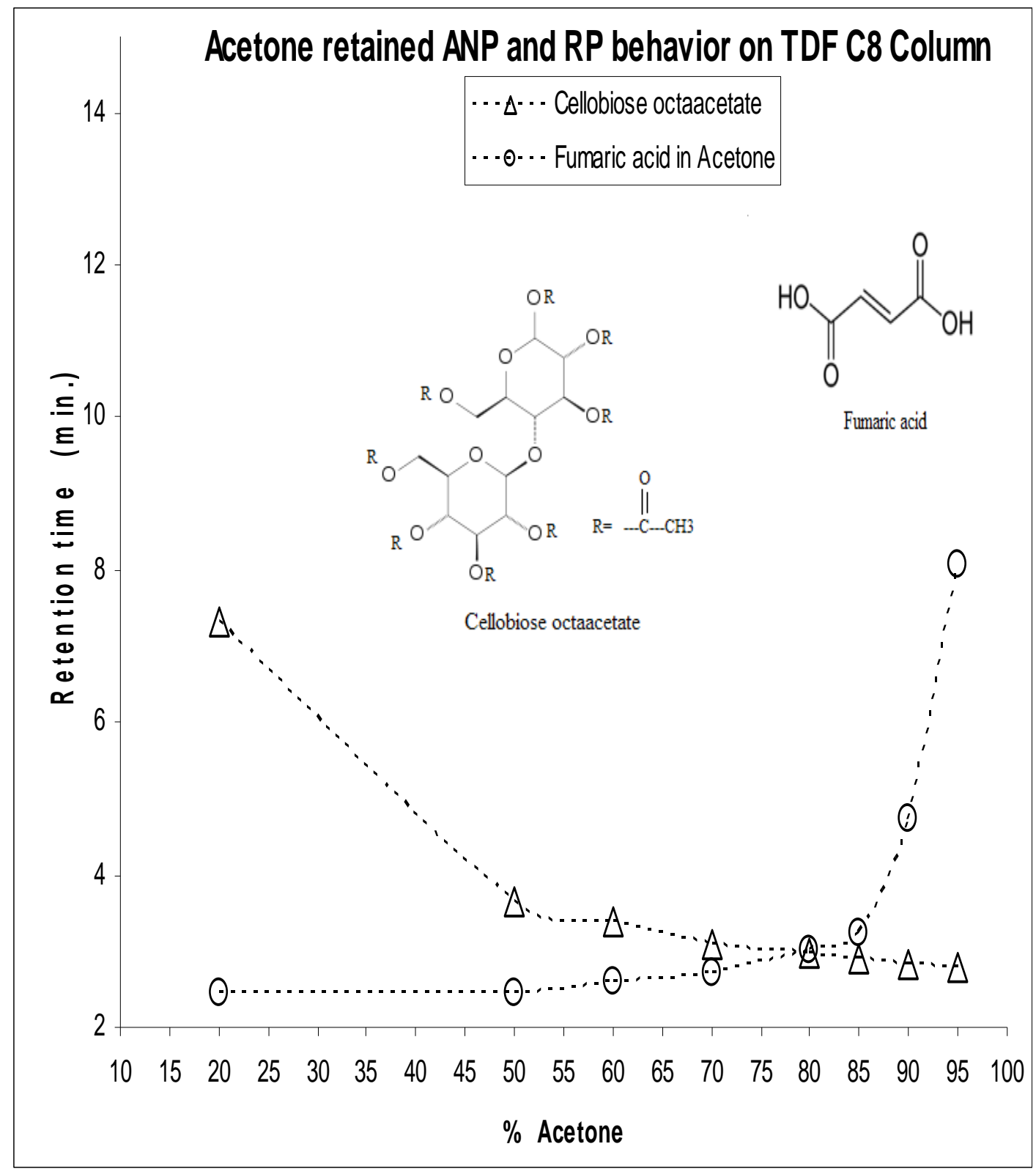

Figure 66. Acetone based ANP behavior of fumaric acid and RP behavior of cellobiose octaacetate on the same column TDF C8. 
Table 33. Comparison of acetonitrile- and acetone- based aqueous normal phase retention of organic acids on the TDF C8 column using LCMS

\begin{tabular}{|c|c|c|c|c|c|}
\hline $\begin{array}{c}\text { Concentration } \\
(\%)\end{array}$ & \multicolumn{2}{|c|}{$\begin{array}{l}\text { Retention time } \\
\text { (min) }\end{array}$} & \multirow{2}{*}{$\begin{array}{c}\begin{array}{c}\text { Concentration } \\
(\%)\end{array} \\
\\
\text { Acetone } \\
+ \\
(15 \mathrm{mM} \\
\text { Ammonium } \\
\text { formate })\end{array}$} & \multicolumn{2}{|c|}{$\begin{array}{l}\text { Retention time } \\
\text { (min) }\end{array}$} \\
\hline $\begin{array}{c}\text { Acetonitrile } \\
+ \\
(15 \mathrm{mM} \\
\text { Ammonium- } \\
\text { formate })\end{array}$ & $\begin{array}{l}\text { Fumaric } \\
\text { acid }\end{array}$ & $\begin{array}{l}\text { Citric } \\
\text { acid }\end{array}$ & & $\begin{array}{l}\text { Fumaric } \\
\text { acid }\end{array}$ & $\begin{array}{l}\text { Citric } \\
\text { acid }\end{array}$ \\
\hline 50 & 2.24 & 2.22 & 50 & 2.46 & 2.43 \\
\hline 60 & 2.36 & 2.32 & 60 & 2.59 & 2.49 \\
\hline 70 & 2.62 & 2.61 & 70 & 2.73 & 2.61 \\
\hline 80 & 3.66 & 3.89 & 80 & 3.00 & 2.92 \\
\hline 85 & 5.05 & 6.62 & 85 & 3.22 & 3.24 \\
\hline 90 & 9.97 & 19.15 & 90 & 4.73 & 6.15 \\
\hline 95 & - & - & 95 & 8.07 & 14.44 \\
\hline
\end{tabular}




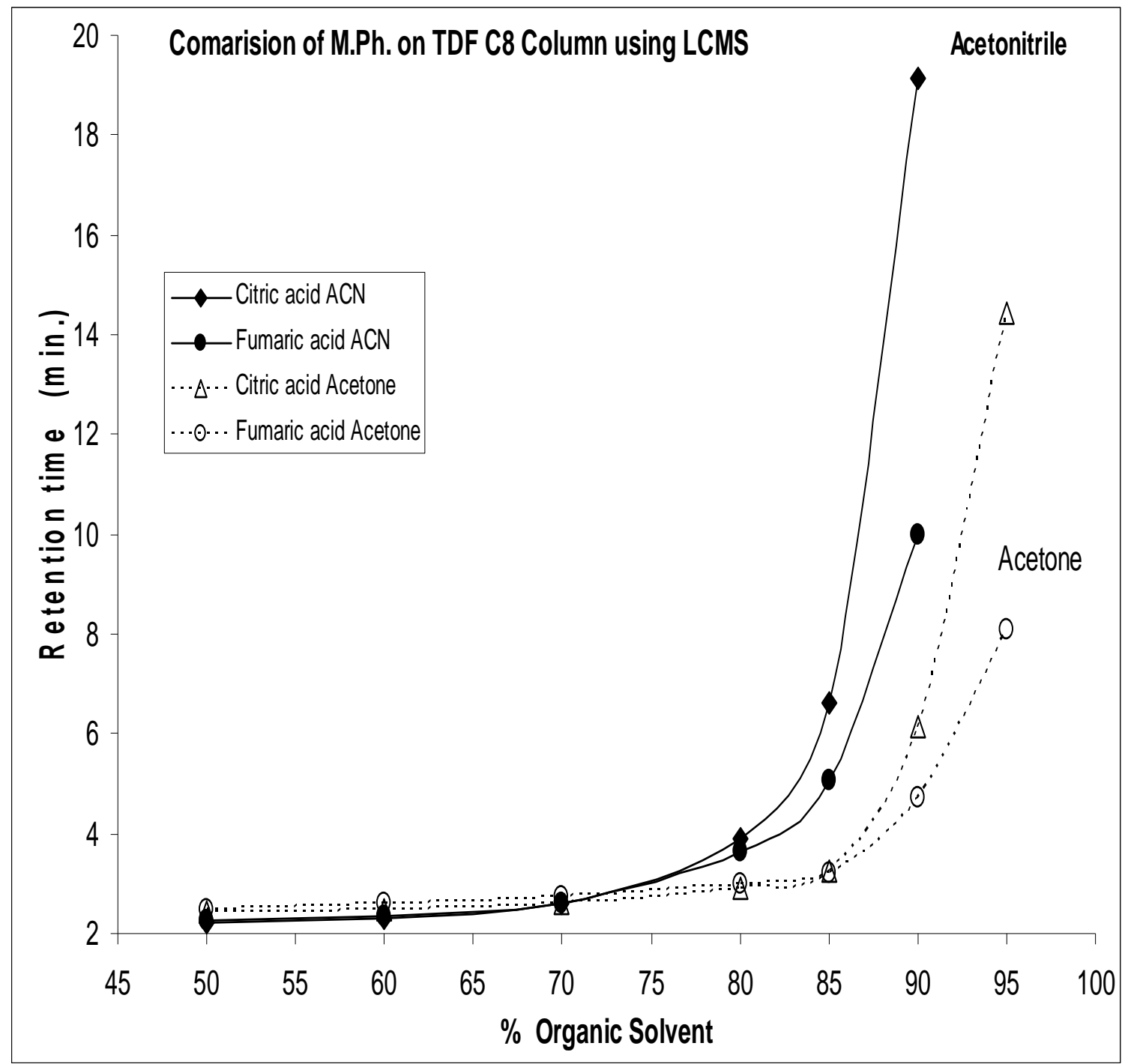

Figure 67. Comparable acetonitrile- based and acetone- based ANP retention maps of organic acids on the TDF C8 column.

Using acetone as the ANP solvent, glucose, fructose, ribose and sucrose were also analyzed on the TDF C8 column for 50:50 to 95:05 acetone:water $\mathrm{pH} \sim 7$ mobile phase composition. However, their SIM chromatograms had very poor signal/noise ratio. 
Concentrations from 0.5 to $3 \mathrm{mg} / \mathrm{mL}$ with varying injection volumes and threshold values were also tried. The nebulization temperature as well as ionization temperature was varied to check for good shaped peaks. Regardless of all the conditions performed on the TDF C8 column, the experimental conditions were not optimized enough to study their ANP retention using acetone. 


\section{CONCLUSIONS}

The synthetic procedures of silanization and hydrosilation have successfully produced silica hydride based fluorinated stationary phases: tridecafluoro-1-octene silica hydride (TDF C8) and heptadecafluoro-1-decene silica hydride (HDF C10). The DIRFT and NMR spectroscopic results have qualitatively confirmed the bonding of the hydride on silica material and the bonding of the carbonaceous fluorinated moiety onto the silicon hydride. The novel slurry state proton-NMR confirmed the replacement of the silanols, the formation of the silica hydride, and the bonding of the fluorocarbonaceous moiety of the TDF C8 and HDF C10 stationary phase materials. The slurry state proton-NMR peaks are consistent with the characteristic predictions for the $\mathrm{Si}-\mathrm{H}$ and $\mathrm{C}-\mathrm{H}$ groups and thus further substantiated the successful bonding of the fluorinated moieties. In addition, these results confirm the results obtained in the DRIFT spectra.

The fluorine-19 slurry state NMR spectra showed a small fluorine peak indicative for both the TDF C8 and HDF C10 materials. This result provides the primary confirmation of the attachment of the fluorinated moiety on to the silica hydride stationary phases.

The carbon elemental analyses of the TDF C8 and HDF C10 materials confirmed the bonding of the fluorocarbonaceous moiety on to the silica hydride quantitatively. On the basis of this result, a hypothesis was made that the higher amount of fluorine moiety on to the TDF C8 will result in greater fluorine based interactions and the comparatively lower amount of fluorine moiety on to the HDF $\mathrm{C} 10$ will provide more interaction with the silica hydride layer for solute interaction. This hypothesis, made for the purpose of 
comparison, was supported by the aqueous normal phase (ANP) retention data obtained on the TDF C8 and HDF C10 columns.

The TDF C8 and HDF C10 columns have shown good ANP behavior for amino acids, nucleobases, nucleosides, nucleotides, ergogenic acids, organic acids, and carbohydrates analyzed using HPLC with ELSD, UV, and MS detectors. The organic solvent acetonitrile $(>60 \%)$ has become the primary component of the ANP binary solvent mobile phase system. However, the nonpolar solvent acetone also resulted in ANP retention for the polar solutes on both columns. Additionally, success in the reversed phase (RP) retention observed in the case of nonpolar cellobiose octaacetate disaccharide, proves the suitability of these fluorinated bonded phases for RP retention.

The trend for the ANP retention of all these solutes has been observed as follows on both columns. For the amino acids: the basic amino acids were retained the most, followed by the neutral, and the acidic amino acids (the polar neutral tyrosine was retained more than neutral phenylalanine). For nucleobases, cytosine was retained the most, followed by guanine and uracil. In the case of nucleosides, adenosine was retained more than thymidine. Whereas, when organic acids were analyzed, citric acid retained the most, followed by fumaric acid, succinic acid, and maleic acid. This general trend of ANP retention deduces that the higher polarity solutes are retained the most on the fluorinated columns.

The use of $0.1 \%$ FA could retain amino acids, nucleobases, nucleosides and ergogenic acids. However, for the retention of a nucleotide, organic acids, and 
carbohydrates, the use of $15 \mathrm{mM}$ ammonium formate provided better ANP retention. Thus, a careful use of additive can increase the ANP retention on the fluorinated columns. To understand the plausible retention mechanism exhibited by the TDF C 8 and HDF C10 columns for different polarity analytes, their ANP retention maps were compared. The solute molecules like histidine, arginine, and tyrosine amino acids were retained more on the TDF C8 column indicating the ANP retention due to fluorinated moiety's hydrophilic interaction. Whereas, phenylalanine and monosaccharides like glucose and fructose were retained more on the HDF C10 column which has more of hydride influence for comparative hydrophobic interaction. However, the nucleotide ATP and citric acid were retained equally on both columns. If we compare the RP retention of cellobiose octaacetate at $80 \%$ water and $20 \%$ acetonitrile mobile phase composition, it retains more on the TDF $\mathrm{C} 8$ suggesting that the fluorinated moiety of TDF C8 is acting as nonpolar stationary phase (exhibiting hydrophobic interaction) to the nonpolar solute. Hence, a fluorinated moiety can provide hydrophilic as well as hydrophobic interaction for different solutes. Further comparison of ANP retention data analyzed on the TDF C8 and HDF C10 columns against that analyzed on pure silica hydride columns of equal dimensions and similar experimental conditions, can afford further insight toward the understanding of the hydride based and fluorine based synergistic retention mechanisms.

The carbohydrates have shown very good ANP retention on both columns. Furthermore, it has been seen that the disaccharides retain more than the monosaccharides on both columns as they have more polar hydroxyl groups. Since these columns provide reproducibility of less than $1 \%$ RSD values, higher precision in data 
obtained for real analyses can be expected. These columns have handled many analyses over a long period of time which confirms its long time stability due to the Si-C bonding of the fluorocarbon moieties. Due to the high stability and reproducibility of these columns, the comparison of the shapes of the retention maps obtained using different detectors was possible. The similarities in the shapes of the retention maps have proved the validity of the retention data obtained using UV and ELSD. Finally, on the basis of the retention maps of the small polar biomolecules, gradient methods can be developed to separate these metabolites and/or to study larger biomolecules. 


\section{REFERENCES}

1. Skoog, A. D.; Holler, F. J.; Nieman, T. A. Principles of Instrumental Analysis, $5^{\text {th }}$ ed.; Saunders College Publishing: Orlando, Florida, 1998; Chapters 26, 28.

2. Cunico, R. L.; Gooding, K. M.; Wehr, T. Basic HPLC and CE of Biomolecules, Bay Bioanalytical Laboratory, CA, 1998.

3. Megoulas, N. C.; Koupparis, M. A. Critical Reviews in Analytical Chemistry. 35, 301-316, 2005.

4. SOFTA CORPORATION, Model 400 ELSD Manual, version 1.2, August 2006. http://www.softacorporation.com (accessed November $2^{\text {nd }}, 2009$ ).

5. Applied Biosystems,/ MDS Sciex, Operator's Manual, P/N 1004314A, July 2002.

6. Pesek, J.J.; Matyska, M.T. LCGC NORTH AMERICA. 2006, 24, 3, 296-303.

7. Pesek, J. J.; Matyska, M. T.; Venkat, J. P. J. Sep. Sci. 2008, 31, 2560-2566.

8. Pesek, J. J.; Matyska, M. T.; Fischer, S. M.; Sana, T. R. J. Chromatogr. A. 2008, 1204, 48-55.

9. Pesek, J. J.; Matyska, M. T. J. Sep. Sci. 2005, 28, 1845-1854.

10. Pesek, J. J.; Matyska, M. T. Studies in Surface Science and Catalysis. 1999, 120, 117-142.

11. Nawrocki, J. J. Chromgra. A. 1997, 779, 29-71.

12. Pesek, J. J.; Matyska, M. T. Interface Science. 1997, 5, 103-117.

13. Pesek, J. J.; Matyska, M.T. J. Liq. Chromgra. Rel. Technol. 2006, 29, $1105-$ 1124.

14. Euerby, M. R.; McKeown, A. P.; Petersson, P. J. Sep. Sci. 2003, 26, 295-306.

15. Przybyciel, M. LCGC Europe. 2006, 19, 1, 1-10.

16. Zhang, W. Journal of Fluorine Chemistry. 2008, 129, 910-919.

17. Xindu, G.; Carr, P. W. J. Chromatogra. 1983, 269, 96-102. 
18. Jinno, K.; Nakamura, H. Chromatographia. 1994, 39, 5/6, 285-293.

19. Haas, A.; Kohler, J. Chromatographia. 1981, 14, 6, 341-344.

20. Berendsen, G. E.; Pikaart, K. A.; De Galan, L. Anal. Chem. 1980, 52, 12, 19901993.

21. Pesek, J. J.; Matyska, M. T.; Hearn, M. T. W.; Boysen, R. I. J. Chromatogra. A 2009, 1216, 1140-1146.

22. Kay Ernest, R. M.; Biochemistry- An Introduction to Dynamic Biology. Macmillan Company: NY, 1996; Chapter 26, pp 342.

23. Wolinsky. I.; Driskell. J. Nutritional Ergogenic Acids, CRC Press: NY, 2004; Chapter 6, pp 81-104. 